Supporting Information

\title{
Lewis Acid Activation of Fragment-Coupling Reactions of Tertiary Carbon Radicals Promoted by Visible Light Irradiation of EDA Complexes
}

\author{
Spencer P. Pitre $\$$, Tyler K. Allred ${ }^{\ddagger}$ \& Larry E. Overman* \\ Department of Chemistry, University of California, Irvine, California 92697-2025 \\ Email: Larry E. Overman: leoverma@uci.edu
}


Table of Contents

A. Materials and Methods..................................................... S3

B. Pictures of Photobox Experimental Setup...................................... S4

C. Literature on Coupling of Tertiary Nucleophiles with Conjugated Cyclic Enones.................................................................................

D. Literature Comparisons on the Addition of Tertiary Radicals to 5-7 Membered Conjugated Cyclic Enones......................................... S6

E. Synthesis of Cyclic Enone Substrates....................................... S7

F. Synthesis of N-Hydroxyphthalimide (NHPI) Esters.......................... S8

G. Summary of Reaction Optimization Experiments............................ S10

H. General Procedure for the Gd(OTf) $)_{3}$-Assisted Giese Coupling Reaction.......... S13

I. Reaction Scope of the Gd(OTf) 3 -Assisted Giese Coupling Reaction.............. S14

J. Procedure for the Coupling of Moderately Complex Fragments................ S29

K. Large Scale Fragment Coupling Procedure................................... S31

L. Benesi-Hildebrand Analysis of NHPI Ester-HE EDA Complex................. S32

M. Conversion vs. Time Experiments.............................................. S34

N. Unsuccessful or Low Yielding Scope Examples................................ S38

O. Syntheses of Substrates that Coupled in Low Yield............................ S39

P. X-Ray Structure of Giese Adduct 21....................................... S46

Q. References................................................................. S48

R. NMR Spectra......................................................... S49 


\section{A. Materials and Methods}

Unless stated otherwise, reactions were conducted in oven-dried glassware under an atmosphere of argon. Tetrahydrofuran (THF), 1,2-dimethoxyethane (DME), dimethylformamide (DMF), toluene, dichloromethane $\left(\mathrm{CH}_{2} \mathrm{Cl}_{2}\right)$, methanol $(\mathrm{MeOH})$ and triethylamine were dried by passage through activated alumina. All commercial reagents were used as received unless otherwise noted. Reactions carried out at elevated temperature were conducted by partially immersing the flask in a controlled-temperature sand bath. Unless stated otherwise, reactions were performed at room temperature (rt, approximately $23{ }^{\circ} \mathrm{C}$ ). Thin-layer chromatography (TLC) was conducted with silica gel $60 \mathrm{~F} 254$ pre-coated plates, $(0.25 \mathrm{~mm})$ and visualized by exposure to UV light $(254 \mathrm{~nm})$ or by $p$-anisaldehyde, ceric ammonium molybdate (CAM), and potassium permanganate $\left(\mathrm{KMnO}_{4}\right)$ staining. Silica gel 60 (particle size $0.040-0.063 \mathrm{~mm}$ ) was used for flash column chromatography. ${ }^{1} \mathrm{H}$ NMR spectra were recorded at 500 or $600 \mathrm{MHz}$ and are reported relative to deuterated solvent signals. Data for ${ }^{1} \mathrm{H}$ NMR spectra are reported as follows: chemical shift $(\delta \mathrm{ppm})$, multiplicity, coupling constant $(\mathrm{Hz})$, and integration. ${ }^{13} \mathrm{C}$ NMR spectra were recorded at 125 or $150 \mathrm{MHz}$. Data for ${ }^{13} \mathrm{C}$ NMR spectra are reported in terms of chemical shift. IR spectra were recorded on a ThermoFisher Nicolet iS5 FT-IR spectrometer with an iD7 ATR accessory and are reported in terms of frequency of absorption $\left(\mathrm{cm}^{-1}\right)$. High-resolution mass spectra were obtained with an LCT spectrometer. UV-Vis spectra were recorded on a Jasco v-530 $\mathrm{UV}$-Vis spectrophotometer. Optical rotations were measured with a Jasco P-1010 polarimeter. Kessil KSH150B LED Grow Light 150, Blue (34 W blue LED lamps) were purchased from http://www.amazon.com. See JOC Standard Abbreviations and Acronyms for abbreviations (http://pubs.acs.org/paragonplus/submission/joceah/joceah_abbreviations.pdf) 


\section{B. Pictures of Photobox Experimental Setup}

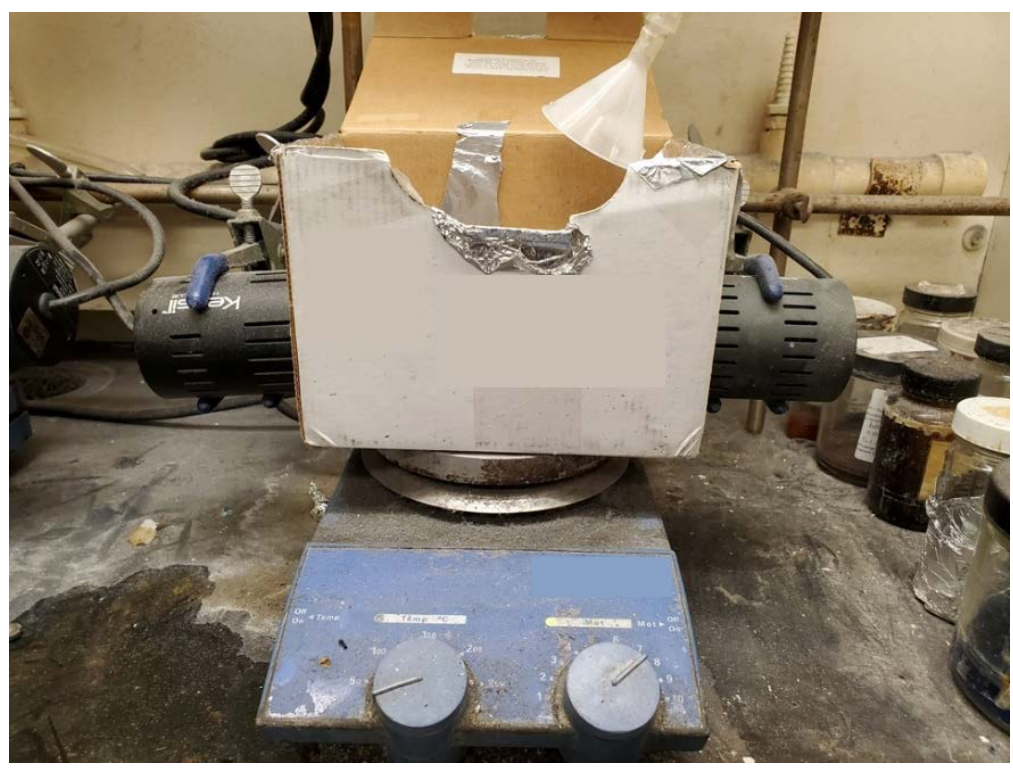

Figure S1. Side view. Box length: 7 inches. One 34 W Kessil KSH150B Blue LED Grow Light 150 is positioned on each side. A tube is positioned above the reaction box to blow air in order to cool the interior of the photobox.

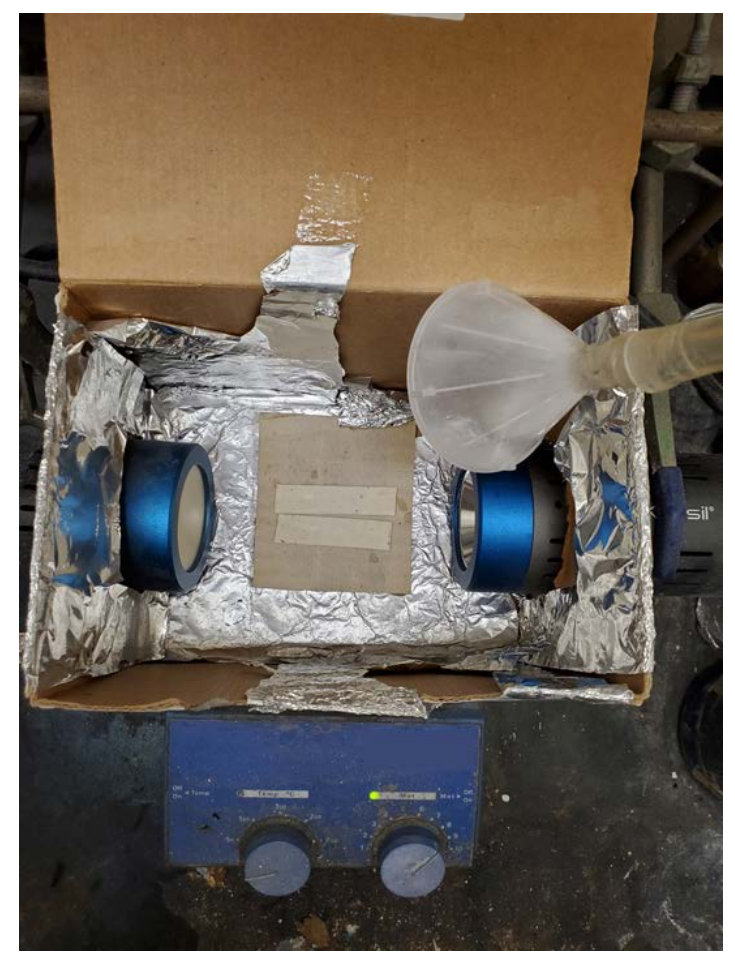

Figure S2. Top view.

Photographs were taken in the Overman Laboratory by Tyler Allred 


\section{Literature on Coupling of Tertiary Nucleophiles with Conjugated Cyclic Enones}

The depicted reaction search was conducted in SciFinder for additions to 5-, 6-, 7-, and 8membered cyclic enones. Only bimolecular examples reported in journal articles were considered. Those involving the addition of a tertiary radical were then selected by examining each hit. Almost all non-radical examples involved the 1,4-addition of an organometallic nucleophile (most commonly a cuprate). The following is a summary of this analysis:

2-cyclopenten-1-one: 46 examples, 29 of which were radical additions 2-cyclohexen-1-one: 85 examples, 11 of which were radical additions 2-cyclohepten-1-one: 20 examples, 2 of which were radical additions 2-cycloocten-1-one: 2 examples, none of which were radical additions

Task History

Task Began December 22, 2020 05:22 PM

Explore reactions by substructure (ID 2)
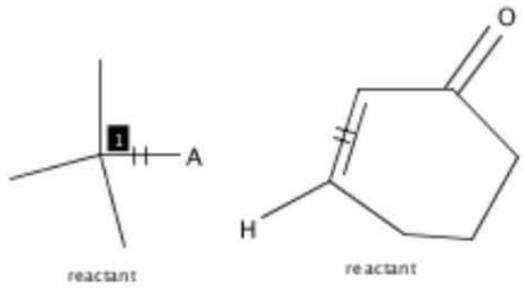

reacunt reactant
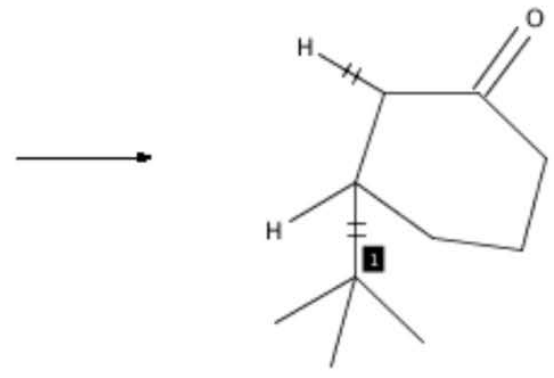

Limiters:

Source:

Number of Steps:

Any source

Answer Type:

Reactions

Result Count:

116 


\section{Literature Comparisons on the Addition of Tertiary Radicals to 5-7 Membered Conjugated Cyclic Enones}

\section{A. Overman \& Coworkers ${ }^{1}$}<smiles>CC1(OC(=O)C(=O)ON2C(=O)c3ccccc3C2=O)CCCCC1</smiles>

1.5 equiv<smiles>O=C1C=CCC1</smiles>

1.0 equiv

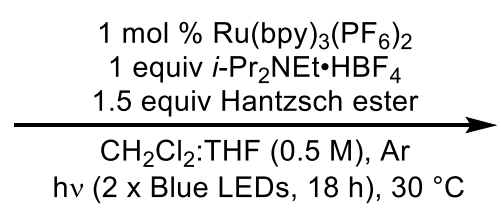

$\mathrm{h} v(2 \times$ Blue LEDs, $18 \mathrm{~h}), 30^{\circ} \mathrm{C}$

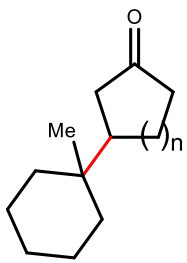

$\mathrm{n}=1: 54 \%$ $\mathrm{n}=2: 15 \%$

\section{B. Baran \& Coworkers ${ }^{2}$}

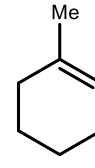

$+$<smiles>O=C1C=CC=C1</smiles>

1.0 equiv
$40 \mathrm{~mol} \% \mathrm{Fe}(\mathrm{acac})_{3}$

1.5 equiv $\mathrm{PhSiH}_{3}$

5:1 DCE: $\left(\mathrm{CH}_{2} \mathrm{OH}\right)_{2}, 60^{\circ} \mathrm{C}$

\section{0 equiv}

C. Baran \& Coworkers ${ }^{3}$<smiles>CC1(OC(=O)C(=O)ON2C(=O)c3ccccc3C2=O)CCNCC1</smiles><smiles>O=C1C=CC=C1</smiles>

2 equiv $\mathrm{Zn}, 3$ equiv $\mathrm{LiCl}$ $20 \mathrm{~mol} \% \mathrm{Ni}\left(\mathrm{ClO}_{4}\right) \cdot 6 \mathrm{H}_{2} \mathrm{O}$ $\mathrm{MeCN}, \mathrm{rt}, 12 \mathrm{~h}$ 2.0 equiv<smiles>CC1(C2CCC(=O)C2)CC[NH2+]CC1</smiles>
$\mathrm{n}=1: 48 \%$ $n=2: 47 \%$

$\mathrm{n}=1: 65 \%$

$\mathrm{n}=2: 38 \%$ (used $100 \mathrm{~mol} \%$ $\left.\mathrm{Fe}(\mathrm{acac})_{3}\right)$

\section{E. Pospech \& Coworkers ${ }^{4}$}<smiles>O=C(O)C12CC3CC(CC(C3)C1)C2</smiles>

1.0 equiv<smiles>O=C1C=CCC1</smiles>

2.0 equiv

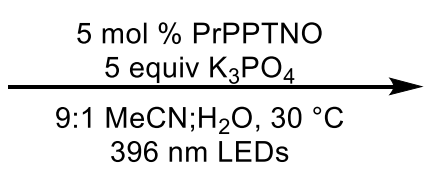
396 nm LEDs<smiles>O=C1CCC(C2C3CC4CC(C3)CC2C4)C1</smiles>

$\mathrm{n}=1: 89 \%$

$\mathrm{n}=2: 81 \%$ $\mathrm{n}=3: 19 \%$

\section{E. Larionov \& Coworkers ${ }^{5}$}<smiles>O=C(O)C12CC3CC(CC(C3)C1)C2</smiles>

1.0 equiv<smiles>O=C1C=CC=C1</smiles>

2.5 equiv
$10 \mathrm{~mol} \% \mathrm{Cu}(\mathrm{MeCN})_{4} \mathrm{BF}_{4}$ $10 \mathrm{~mol} \%$ acridine photocatalyst $10 \mathrm{~mol} \%$ piperazine $\mathrm{CH}_{2} \mathrm{Cl}_{2}$, rt, $14 \mathrm{~h}$ $400 \mathrm{~nm}$ LEDs<smiles>O=C1CC([AlH2])C(C23CC4CC(CC(C4)C2)C3)C1</smiles>

$\mathrm{n}=2: 77 \%$ 


\section{E. Synthesis of Cyclic Enone Substrates}

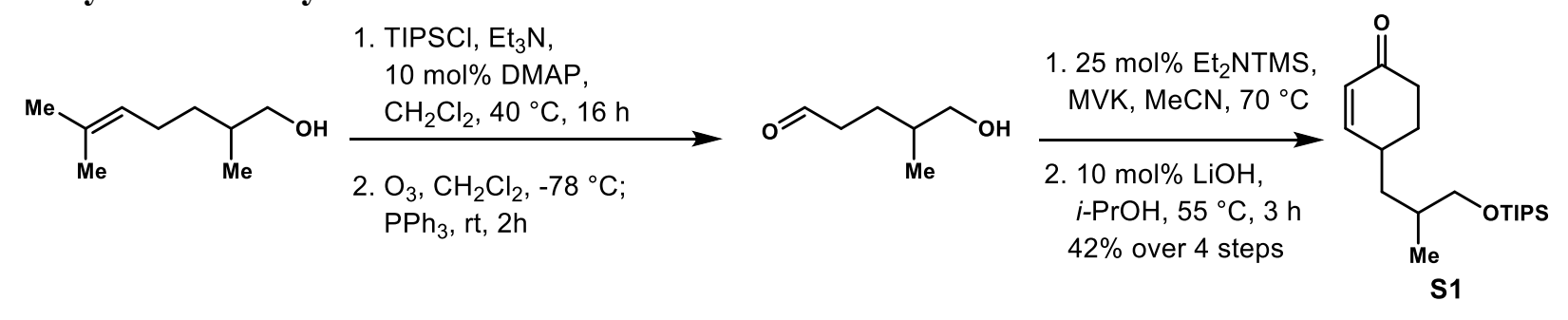

Enone S1: To a solution of the 2,6-dimethyl-5-heptenol $(2.00 \mathrm{~g}, 14.1 \mathrm{mmol})$ in $\mathrm{CH}_{2} \mathrm{Cl}_{2}(30 \mathrm{~mL}$, $0.5 \mathrm{M})$ at rt, $\mathrm{Et}_{3} \mathrm{~N}$ (3.3 mL, $24 \mathrm{mmol}, 1.7$ equiv) and DMAP (171 mg, $1.4 \mathrm{mmol}, 10 \mathrm{~mol} \%$ ) were added. Then TIPSCl ( $4.4 \mathrm{~mL}, 21 \mathrm{mmol}, 1.5$ equiv) was added and the mixture was maintained at $40{ }^{\circ} \mathrm{C}$ for $16 \mathrm{~h}$. The reaction was then cooled to $\mathrm{rt}$ and quenched with sat. aq. $\mathrm{NH}_{4} \mathrm{Cl}(40 \mathrm{~mL})$. The mixture was then extracted with EtOAc $(2 \times 30 \mathrm{~mL})$. The combined organic layers were then washed with brine $(30 \mathrm{~mL})$, dried over $\mathrm{MgSO}_{4}$, filtered, and concentrated. The crude residue was then flushed through a $\mathrm{SiO}_{2}$ plug with hexanes $(200 \mathrm{~mL})$. The filtrate was concentrated to afford the intermediate silyl ether $(3.72 \mathrm{~g}, 89 \%)$, which was used without further purification.

The crude silyl ether was dissolved in $\mathrm{CH}_{2} \mathrm{Cl}_{2}(60 \mathrm{~mL}, 0.2 \mathrm{M})$ and cooled to $-78{ }^{\circ} \mathrm{C}$. Then $\mathrm{O}_{3}$ was bubbled through the reaction mixture until a persistent blue color was observed. Then the reaction was purged with $\mathrm{O}_{2}$ until the blue color dissipated. Then $\mathrm{PPh}_{3}$ (7.25 g, $27.6 \mathrm{mmol}^{2} 2.2$ equiv) was added and the mixture was stirred at $-78^{\circ} \mathrm{C}$ for $1 \mathrm{~h}$ and then allowed to warm to rt over $2 \mathrm{~h}$. The reaction was then concentrated and the crude residue was dissolved in hexanes $(100 \mathrm{~mL})$ and cooled to $0{ }^{\circ} \mathrm{C}$ while stirring. The reaction was then filtered and the solids were washed with cold hexanes. The filtrate was concentrated to afford crude aldehyde, which was used without further purification.

The crude aldehyde was dissolved in MeCN (30 mL, 0.4M) and $\mathrm{Et}_{2} \mathrm{NTMS}(0.59 \mathrm{~mL}, 3.10$ mmol, $25 \mathrm{~mol} \%)$ was added followed by $\mathrm{MVK}(1.5 \mathrm{~mL}, 18.6 \mathrm{mmol}, 1.5$ equiv). The reaction mixture was then heated to $70^{\circ} \mathrm{C}$ for $15 \mathrm{~h}$. The reaction was then cooled to $\mathrm{rt}$ and concentrated to afford the crude Michael adduct. The crude material was taken up in $i-\mathrm{PrOH}(30 \mathrm{~mL}, 0.4 \mathrm{M})$ and $\mathrm{LiOH} \cdot \mathrm{H}_{2} \mathrm{O}(50 \mathrm{mg}, 1.2 \mathrm{mmol}, 10 \mathrm{~mol} \%)$ was added. The reaction was then warmed to $55{ }^{\circ} \mathrm{C}$ for $3 \mathrm{~h}$. The reaction was then cooled to $\mathrm{rt}$ and concentrated. The crude residue was diluted with sat. aq. $\mathrm{NH}_{4} \mathrm{Cl}(100 \mathrm{~mL})$ and extracted with EtOAc $(2 \times 50 \mathrm{~mL})$. The combined organic layers were washed with brine $(50 \mathrm{~mL})$, dried over $\mathrm{MgSO}_{4}$, filtered and concentrated. The crude residue was purified by flash column chromatography $\left(\mathrm{SiO}_{2}, 1: 0 \rightarrow 20: 1 \mathrm{Hex} / \mathrm{EtOAc}\right)$ to afford enone $\mathbf{S 1}$ (1.9 $\mathrm{g}, 42 \%$ over 4 steps) as a colorless oil and 1:1 mixture of diastereomers. $\mathrm{R}_{\mathrm{f}}=0.71(9: 1 \mathrm{Hex} / \mathrm{EtOAc})$

${ }^{1} \mathbf{H}$ NMR $\left(600 \mathrm{MHz}, \mathrm{CDCl}_{3}\right) \delta 6.90-6.82(\mathrm{~m}, 1 \mathrm{H}), 5.97(\mathrm{dd}, J=2.3,10.2 \mathrm{~Hz}, 1 \mathrm{H}), 3.60-3.48(\mathrm{~m}$, $2 \mathrm{H}), 2.58-2.46(\mathrm{~m}, 2 \mathrm{H}), 2.40-2.30(\mathrm{~m}, 1 \mathrm{H}), 2.18-2.08(\mathrm{~m}, 1 \mathrm{H}), 1.84-1.73(\mathrm{~m}, 1 \mathrm{H}), 1.73-1.56(\mathrm{~m}$, $2 \mathrm{H}), 1.32-1.26(\mathrm{~m}, 0.5 \mathrm{H}), 1.22-1.16(\mathrm{~m}, 0.5 \mathrm{H}), 1.12-1.07(\mathrm{~m}, 3 \mathrm{H}), 1.06-1.03(\mathrm{~m}, 18 \mathrm{H}), 0.96(\mathrm{~d}$, $J=6.7 \mathrm{~Hz}, 1.5 \mathrm{H}), 0.94(\mathrm{~d}, J=6.7 \mathrm{~Hz}, 1.5 \mathrm{H})$

${ }^{13}$ C NMR $\left(125 \mathrm{MHz}, \mathrm{CDCl}_{3}\right) \delta 200.1,200.0,156.0,155.2,128.9,68.7,68.5,38.6,38.4,37.0$, $36.8,33.8,33.7,33.4,29.6,28.6,18.1,17.8,17.34,17.31,16.7,12.0$

IR (thin film, cm ${ }^{-1}$ ) 2942, 2865, 1684, 1462, 1388, 1100, 1067, 1013

HRMS (ESI-TOF) m/z: [M+Na] $]^{+}$calcd for $\mathrm{C}_{19} \mathrm{H}_{36} \mathrm{O}_{2} \mathrm{SiNa} 347.2382$, found 347.2386 


\section{F. Synthesis of N-Hydroxyphthalimide (NHPI) Esters}

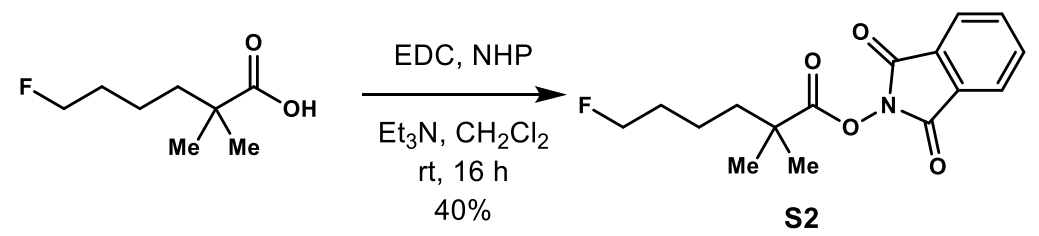

NHPI Ester S2. To a solution of 6-fluoro-2,2-dimethylhexanoic acid 6 (668 $\mathrm{mg}, 4.11 \mathrm{mmol})$ in $\mathrm{CH}_{2} \mathrm{Cl}_{2}(20 \mathrm{~mL}, 0.2 \mathrm{M})$ at rt, $\mathrm{Et}_{3} \mathrm{~N}(1.7 \mathrm{~mL}, 12 \mathrm{mmol}, 3$ equiv) was added followed by $\mathrm{EDC} \cdot \mathrm{HCl}$ ( $1.19 \mathrm{~g}, 6.20 \mathrm{mmol}, 1.5$ equiv). The mixture was stirred for $5 \mathrm{~min}$ and then $\mathrm{N}$-hydroxyphthalimide ( $1.00 \mathrm{~g}, 6.15 \mathrm{mmol}, 1.5$ equiv) was added. The mixture was then stirred at rt overnight ( $\sim 16 \mathrm{~h})$. The reaction was then diluted with $1 \mathrm{M} \mathrm{HCl}(30 \mathrm{~mL})$. The mixture was then extracted with $\mathrm{CH}_{2} \mathrm{Cl}_{2}$ $(2 \times 20 \mathrm{~mL})$. The combined organic layers were then dried over $\mathrm{MgSO}_{4}$, filtered, and concentrated to an orange oil. The crude residue was purified by flash column chromatography $\left(\mathrm{SiO}_{2}, 0: 1 \rightarrow 9: 1\right.$ Hex/EtOAc) to afford NHPI ester S2 (503 mg, 40\%) as a colorless solid. $\mathrm{R}_{\mathrm{f}}=0.44$ (9:1 $\mathrm{Hex} / \mathrm{EtOAc})$

${ }^{1} \mathbf{H}$ NMR $\left(600 \mathrm{MHz}, \mathrm{CDCl}_{3}\right) \delta$ 7.90-7.84 (m, 2H), 7.80-7.76 (m, 2H), $4.49(\mathrm{dt}, J=6.1,47.3 \mathrm{~Hz}$, $2 \mathrm{H}), 1.82-1.71(\mathrm{~m}, 4 \mathrm{H}), 1.60-1.51(\mathrm{~m}, 2 \mathrm{H}), 1.40(\mathrm{~s}, 6 \mathrm{H})$

${ }^{13}$ C NMR $\left(150 \mathrm{MHz}, \mathrm{CDCl}_{3}\right) \delta 173.8,162.2,134.7,129.1,123.9,84.5(\mathrm{C}-\mathrm{F}, 1 \mathrm{JC}-\mathrm{F}=164.4 \mathrm{~Hz})$, 83.4 (C-F, 1JC-F = 164.4 Hz), 42.2, 40.3, $30.8(\mathrm{C}-\mathrm{F}, 2 \mathrm{JC}-\mathrm{F}=19.7 \mathrm{~Hz}), 30.7(\mathrm{C}-\mathrm{F}, 2 \mathrm{JC}-\mathrm{F}=19.7$ $\mathrm{Hz}), 25.1,20.73(\mathrm{C}-\mathrm{F}, 3 \mathrm{JCF}=5.5 \mathrm{~Hz}), 20.69(\mathrm{C}-\mathrm{F}, 3 \mathrm{JCF}=5.5 \mathrm{~Hz}), 18.9$

IR (thin film, $\mathrm{cm}^{-1}$ ) 2970, 1807, 1782, 1742, 1467, 1369, 1186, 1132, 1048, 878, 696

HRMS (ESI-TOF) m/z: [M+Na] $]^{+}$calcd for $\mathrm{C}_{16} \mathrm{H}_{18} \mathrm{FNO}_{4} \mathrm{Na} 330.1118$, found 330.1127

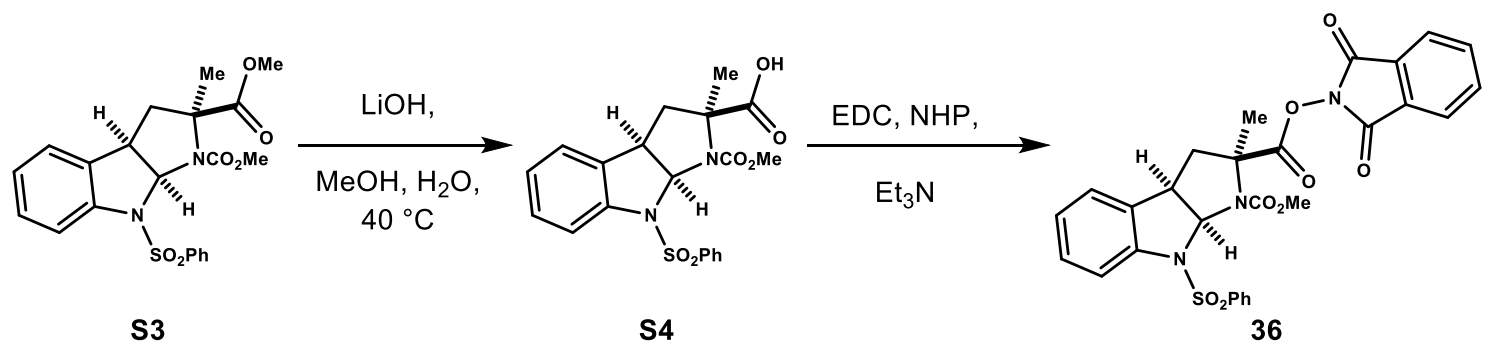

NHPI Ester 36: A $100 \mathrm{~mL}$ round-bottom flask was charged with ester $\mathbf{S 3}^{7}$ (1.06 g, $\left.2.45 \mathrm{mmol}\right)$ in a 1:1 MeOH/ $\mathrm{H}_{2} \mathrm{O}$ mixture $(25 \mathrm{~mL}, 0.1 \mathrm{M}) . \mathrm{LiOH} \cdot \mathrm{H}_{2} \mathrm{O}(1.03 \mathrm{~g}, 24.54 \mathrm{mmol}, 10$ equiv) was added. The mixture was then warmed to $45^{\circ} \mathrm{C}$ for $48 \mathrm{~h}$. The reaction was then cooled to rt and the $\mathrm{MeOH}$ was removed under reduced pressure. The mixture was then acidified with $1 \mathrm{M}$ aq. $\mathrm{HCl}(30 \mathrm{~mL})$ affording a cloudy mixture, which was then washed with $\mathrm{CH}_{2} \mathrm{Cl}_{2}(30 \mathrm{~mL}$ x 3$)$. The combined organic layers were then dried over $\mathrm{MgSO}_{4}$, filtered, and concentrated under reduced pressure to afford crude acid S4 (909 $\mathrm{mg}, 89 \%$ ) as an orange foam. This material was used without further purification.

A $100 \mathrm{~mL}$ round-bottom flask was charged with this crude acid (909 $\mathrm{mg}, 2.18 \mathrm{mmol})$ in $\mathrm{CH}_{2} \mathrm{Cl}_{2}(11 \mathrm{~mL}, 0.2 \mathrm{M})$. EDC (628 mg, $3.27 \mathrm{mmol}, 1.5$ equiv) and $\mathrm{Et}_{3} \mathrm{~N}$ (0.6 mL, $4 \mathrm{mmol}, 2$ equiv) 
were added and the mixture was stirred at $\mathrm{rt}$ for $5 \mathrm{~min}$. Then $\mathrm{N}$-hydroxyphthalimide $(535 \mathrm{mg}, 3.28$ mmol, 1.5 equiv) was added. The resulting red mixture was stirred at $\mathrm{rt}$ for $16 \mathrm{~h}$. The reaction mixture was then poured into a separatory funnel containing $1 \mathrm{M} \mathrm{HCl}(30 \mathrm{~mL})$. The mixture was then extracted with DCM (20 mL x 3). The combined organic layers were then dried over $\mathrm{MgSO}_{4}$, filtered, and concentrated under reduced pressure to afford an orange foam. The crude residue was purified by flash column chromatography $\left(\mathrm{SiO}_{2}, 4: 1 \rightarrow 1: 1 \mathrm{Hex} / \mathrm{EtOAc}\right)$ to afford ester 36 (920 mg, $75 \%)$ as an off-white foam. $\mathrm{R}_{\mathrm{f}}=0.26(1: 1 \mathrm{Hex} / \mathrm{EtOAc})$

${ }^{1} \mathbf{H} \mathbf{N M R}\left(500 \mathrm{MHz}, \mathrm{CDCl}_{3}\right) \delta 7.78(\mathrm{dd}, J=3.1,5.5 \mathrm{~Hz}, 2 \mathrm{H}), 7.72(\mathrm{dd}, J=3.1,5.5 \mathrm{~Hz}, 2 \mathrm{H}), 7.67$ (br d, $J=6.4 \mathrm{~Hz}, 2 \mathrm{H}), 7.51-7.45(\mathrm{~m}, 1 \mathrm{H}), 7.45-7.40(\mathrm{~m}, 1 \mathrm{H}), 7.39-7.34(\mathrm{~m}, 2 \mathrm{H}), 7.19-7.13$ (m, 2H), 7.10-7.04 (m, 1H), 6.29 (d, $J=6.3 \mathrm{~Hz}, 1 \mathrm{H}), 3.67$ (br s, 3H), 3.54 (app t, $J=6.8 \mathrm{~Hz}, 1 \mathrm{H}), 3.03$ $(\mathrm{d}, J=14.0 \mathrm{~Hz}, 1 \mathrm{H}), 2.44(\mathrm{dd}, J=7.8,14.0 \mathrm{~Hz}, 1 \mathrm{H}), 1.89(\mathrm{br} \mathrm{s}, 3 \mathrm{H})$

${ }^{13} \mathrm{C}$ NMR $\left(125 \mathrm{MHz}, \mathrm{CDCl}_{3}\right) \delta 168.7,161.1,153.8,141.5,139.2,134.6,133.1,132.9,128.9$, $128.8,126.9,126.0,124.6,123.8,118.9,81.5,65.0,52.6,43.6,42.4,25.7$

IR (thin film, $\mathrm{cm}^{-1}$ ) 2952, 1818, 1789, 1742, 1716, 1477, 1460, 1445, 1361, 1273, 1217, 1170, $1131,1072,1027$

HRMS (ESI-TOF) m/z: $[\mathrm{M}+\mathrm{Na}]^{+}$calcd for $\mathrm{C}_{28} \mathrm{H}_{23} \mathrm{~N}_{3} \mathrm{O}_{8} \mathrm{SNa} 584.1104$, found 584.1108

Optical Rotation $[\alpha]^{22.0} \mathrm{D}+22.7,[\alpha]^{22.0}{ }_{577}+23.3,[\alpha]^{22.1}{ }_{546}+24.3,[\alpha]^{22.1} 435+30.0\left(c=1.0, \mathrm{CHCl}_{3}\right)$ 


\section{G. Summary of Reaction Optimization Experiments}

Table S1. Selected Giese-coupling optimization and control reactions ${ }^{[a]}$

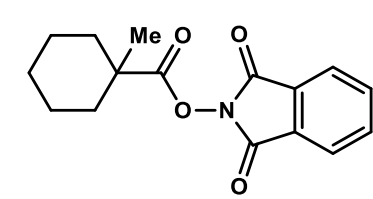

4

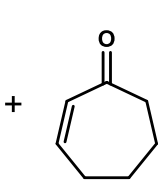

5
Photocatalyst (1 mol\%)

Additive
HE (1.2-1.5 equiv)

Solvent (0.1M), $\mathrm{Ar}, 30^{\circ} \mathrm{C}$

hv (Blue LEDs, $18 \mathrm{~h}$ )

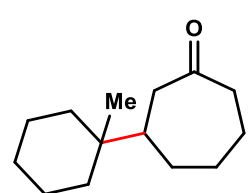

6

\begin{tabular}{|c|c|c|c|c|}
\hline entry & photocatalyst & additive (mol \%) & solvent & yield $^{[b]}$ \\
\hline $1^{[\mathrm{c}]}$ & $\mathrm{Ru}(\mathrm{bpy})_{3}\left(\mathrm{PF}_{6}\right)_{2}$ & $i$ - $\operatorname{Pr}_{2}$ NEt $(220)$ & $\mathrm{CH}_{2} \mathrm{Cl}_{2}$ & $21 \%$ \\
\hline $2^{[\mathrm{c}]}$ & $\mathrm{Ru}(\mathrm{bpy})_{3}\left(\mathrm{PF}_{6}\right)_{2}$ & $\mathrm{La}(\mathrm{OTf})_{3}(75)$ & $\mathrm{CH}_{2} \mathrm{Cl}_{2}$ & $42 \%$ \\
\hline $3^{[\mathrm{c}]}$ & $\mathrm{Ru}(\mathrm{bpy})_{3}\left(\mathrm{PF}_{6}\right)_{2}$ & $\mathrm{La}(\mathrm{OTf})_{3}(75)$ & THF & $76 \%$ \\
\hline $4^{[c]}$ & $\mathrm{Ru}(\mathrm{bpy})_{3}\left(\mathrm{PF}_{6}\right)_{2}$ & $\mathrm{Gd}(\mathrm{OTf})_{3}(50)$ & THF & $70 \%$ \\
\hline $5^{[\mathrm{d}]}$ & None & $\mathrm{Gd}(\mathrm{OTf})_{3}(50)$ & THF & $70 \%$ \\
\hline $6^{[\mathrm{d}]}$ & None & None & THF & $6 \%$ \\
\hline $7^{[\mathrm{d}, \mathrm{e}]}$ & None & $\mathrm{Gd}(\mathrm{OTf})_{3}(50)$ & THF & $0 \%$ \\
\hline $8^{[\mathrm{d}, \mathrm{f}]}$ & None & $\mathrm{Gd}(\mathrm{OTf})_{3}(50)$ & THF & $0 \%$ \\
\hline $9^{[\mathrm{d}]}$ & None & $\mathrm{Yb}(\mathrm{OTf})_{3}(50)$ & THF & $68 \%$ \\
\hline $10^{[\mathrm{d}, \mathrm{g}]}$ & None & $\mathrm{Gd}(\mathrm{OTf})_{3}(50)$ & THF & $62 \%$ \\
\hline
\end{tabular}

[a] Reaction conditions: NHPI ester $4(0.15 \mathrm{mmol})$, enone 5 ( 1.5 equiv), HE (1.2-1.5 equiv), and solvent (0.1 M) were irradiated under an Ar atmosphere using 2 x $34 \mathrm{~W}$ Blue LEDs at $30^{\circ} \mathrm{C}$. [b] Yield determined by ${ }^{1} \mathrm{H}$ NMR analysis using 1,2-dibromo-4,5methylenedioxybenzene as an external standard. [c] Reaction time $=18 \mathrm{~h}$. [d] Reaction time $=2 \mathrm{~h}$. [e] Reaction conducted in the dark. [f] Reaction conducted without HE. [g] Reaction conducted with equimolar amounts of $\mathbf{4}$ and $\mathbf{5}$. 
Table S2. Complete Summary of Giese-coupling reaction optimization.

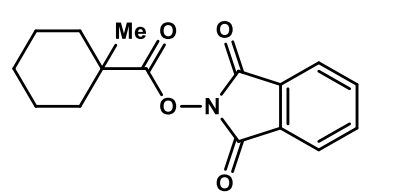

$0.15 \mathrm{mmol}$

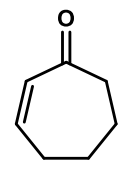

1.5 equiv

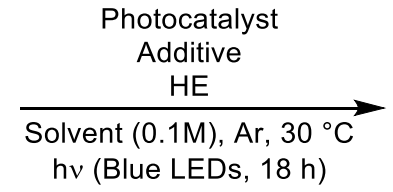

hv (Blue LEDs, $18 \mathrm{~h}$ )

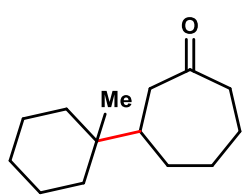

Solvent

Time Yield

Additive (Loading) _ Equiv HE

$i$ - $\operatorname{Pr}_{2} \mathrm{NEt}$ (2.2 equiv)

$i-\mathrm{Pr}_{2} \mathrm{NEt} \cdot \mathrm{HBF}_{4}$

(2.2 equiv)

1.5

1.5

1.5

1.5

1.5

1.5

$\mathrm{Ru}(\mathrm{bpy})_{3}\left(\mathrm{PF}_{6}\right)_{2}(1 \mathrm{~mol} \%)$

$\mathrm{Sc}(\mathrm{OTf})_{3}(25 \mathrm{~mol} \%)$

$\mathrm{Zn}(\mathrm{OTf})_{2}(25 \mathrm{~mol} \%)$

1.5

1.5

1.5

1.5

1.5

1.5

1.5

1.5

1.5

1.5

1.5

1.5

1.5

1.5

$\mathrm{La}(\mathrm{OTf})_{3}(10 \mathrm{~mol} \%)$

1.5

1.5

1.5

1.5

1.5

1.5

1.5

1.5

1.5

$(1 \mathrm{~mol} \%)$

$\mathrm{La}(\mathrm{OTf})_{3}(75 \mathrm{~mol} \%)$

1.5

1.5

1.5

\begin{tabular}{lll} 
THF & $18 \mathrm{~h}$ & $69 \%$ \\
\hline THF & $18 \mathrm{~h}$ & $63 \%$ \\
\hline
\end{tabular}




\begin{tabular}{|c|c|c|c|c|c|c|}
\hline 29 & $\operatorname{Ir}[\mathrm{dFppy}]_{2}(\mathrm{dtbbpy}) \mathrm{PF}_{6}(1 \mathrm{~mol} \%)$ & $\mathrm{La}(\mathrm{OTf})_{3}(75 \mathrm{~mol} \%)$ & 1.5 & THF & $18 \mathrm{~h}$ & $66 \%$ \\
\hline 30 & $\begin{array}{c}\operatorname{Ir}[\mathrm{dF}(\mathrm{Me}) \mathrm{ppy}]_{2}(\mathrm{dtbbpy}) \mathrm{PF}_{6} \\
(1 \mathrm{~mol} \%)\end{array}$ & $\mathrm{La}(\mathrm{OTf})_{3}(75 \mathrm{~mol} \%)$ & 1.5 & THF & $18 \mathrm{~h}$ & $67 \%$ \\
\hline 31 & $\mathrm{Ru}(\mathrm{bpy})_{3}\left(\mathrm{PF}_{6}\right)_{2}(0.5 \mathrm{~mol} \%)$ & $\mathrm{La}(\mathrm{OTf})_{3}(75 \mathrm{~mol} \%)$ & 1.5 & THF & $18 \mathrm{~h}$ & $69 \%$ \\
\hline 32 & 4-CzIPN (2 mol \%) & $\mathrm{La}(\mathrm{OTf})_{3}(75 \mathrm{~mol} \%)$ & 1.5 & THF & $18 \mathrm{~h}$ & $65 \%$ \\
\hline 33 & $\mathrm{Ru}(\mathrm{bpy})_{3}\left(\mathrm{PF}_{6}\right)_{2}(1 \mathrm{~mol} \%)$ & $\mathrm{Gd}(\mathrm{OTf})_{3}(75 \mathrm{~mol} \%)$ & 1.5 & THF & $18 \mathrm{~h}$ & $75 \%$ \\
\hline 34 & 4-CzIPN (1 mol \%) & $\mathrm{Gd}(\mathrm{OTf})_{3}(75 \mathrm{~mol} \%)$ & 1.5 & THF & $18 \mathrm{~h}$ & $64 \%$ \\
\hline 35 & 4-CzIPN (1 mol \%) & $\mathrm{Gd}(\mathrm{OTf})_{3}(50 \mathrm{~mol} \%)$ & 1.5 & THF & $18 \mathrm{~h}$ & $61 \%$ \\
\hline 36 & 4-CzIPN (1 mol \%) & $\mathrm{Gd}(\mathrm{OTf})_{3}(25 \mathrm{~mol} \%)$ & 1.5 & THF & $18 \mathrm{~h}$ & $35 \%$ \\
\hline 37 & $\mathrm{Ru}(\mathrm{bpy})_{3}\left(\mathrm{PF}_{6}\right)_{2}(1 \mathrm{~mol} \%)$ & $\mathrm{Gd}(\mathrm{OTf})_{3}(50 \mathrm{~mol} \%)$ & 1.5 & THF & $18 \mathrm{~h}$ & $70 \%$ \\
\hline 38 & $\mathrm{Ru}(\mathrm{bpy})_{3}\left(\mathrm{PF}_{6}\right)_{2}(1 \mathrm{~mol} \%)$ & $\mathrm{Gd}(\mathrm{OTf})_{3}(50 \mathrm{~mol} \%)$ & 1.0 & THF & $18 \mathrm{~h}$ & $64 \%$ \\
\hline 39 & $\mathrm{Ru}(\mathrm{bpy})_{3}\left(\mathrm{PF}_{6}\right)_{2}(1 \mathrm{~mol} \%)$ & $\mathrm{Gd}(\mathrm{OTf})_{3}(50 \mathrm{~mol} \%)$ & 1.1 & THF & $18 \mathrm{~h}$ & $64 \%$ \\
\hline 40 & $\mathrm{Ru}(\mathrm{bpy})_{3}\left(\mathrm{PF}_{6}\right)_{2}(1 \mathrm{~mol} \%)$ & $\mathrm{Gd}(\mathrm{OTf})_{3}(50 \mathrm{~mol} \%)$ & 1.2 & THF & $18 \mathrm{~h}$ & $71 \%$ \\
\hline 41 & $\mathrm{Ru}(\mathrm{bpy})_{3}\left(\mathrm{PF}_{6}\right)_{2}(1 \mathrm{~mol} \%)$ & $\mathrm{Gd}(\mathrm{OTf})_{3}(50 \mathrm{~mol} \%)$ & 1.2 & THF & $1 \mathrm{~h}$ & $67 \%$ \\
\hline 42 & None & $\mathrm{Gd}(\mathrm{OTf})_{3}(50 \mathrm{~mol} \%)$ & 1.2 & THF & $1 \mathrm{~h}$ & $66 \%$ \\
\hline 43 & None & $\operatorname{Gd}(\mathrm{OTf})_{3}(50 \mathrm{~mol} \%)$ & 1.2 & THF & $2 \mathrm{~h}$ & $70 \%$ \\
\hline 44 & None & $\operatorname{In}(\mathrm{OTf})_{3}(50 \mathrm{~mol} \%)$ & 1.2 & THF & $2 \mathrm{~h}$ & $12 \%$ \\
\hline 45 & None & $\mathrm{Hf}(\mathrm{OTf})_{3}(50 \mathrm{~mol} \%)$ & 1.2 & THF & $2 \mathrm{~h}$ & $3 \%$ \\
\hline 46 & None & $\mathrm{Sc}(\mathrm{OTf})_{3}(50 \mathrm{~mol} \%)$ & 1.2 & THF & $2 \mathrm{~h}$ & $22 \%$ \\
\hline 47 & None & $\mathrm{Yb}(\mathrm{OTf})_{3}(50 \mathrm{~mol} \%)$ & 1.2 & THF & 2 h & $68 \%$ \\
\hline 48 & None & $\operatorname{Dy}(\mathrm{OTf})_{3}(50 \mathrm{~mol} \%)$ & 1.2 & THF & $2 \mathrm{~h}$ & $25 \%$ \\
\hline 49 & None & $\mathrm{Eu}(\mathrm{OTf})_{3}(50 \mathrm{~mol} \%)$ & 1.2 & THF & $2 \mathrm{~h}$ & $8 \%$ \\
\hline 50 & None & $\mathrm{Er}(\mathrm{OTf})_{3}(50 \mathrm{~mol} \%)$ & 1.2 & THF & $2 \mathrm{~h}$ & $49 \%$ \\
\hline 51 & None & $\mathrm{Lu}(\mathrm{OTf})_{3}(50 \mathrm{~mol} \%)$ & 1.2 & THF & $2 \mathrm{~h}$ & $37 \%$ \\
\hline
\end{tabular}

${ }^{\mathrm{a}}$ Yields determined by ${ }^{1} \mathrm{H}$ NMR spectroscopic analysis using 1,2-dibromo-4,5-methylenedioxybenzene as an external standard.

Table S3. Concentration screen.

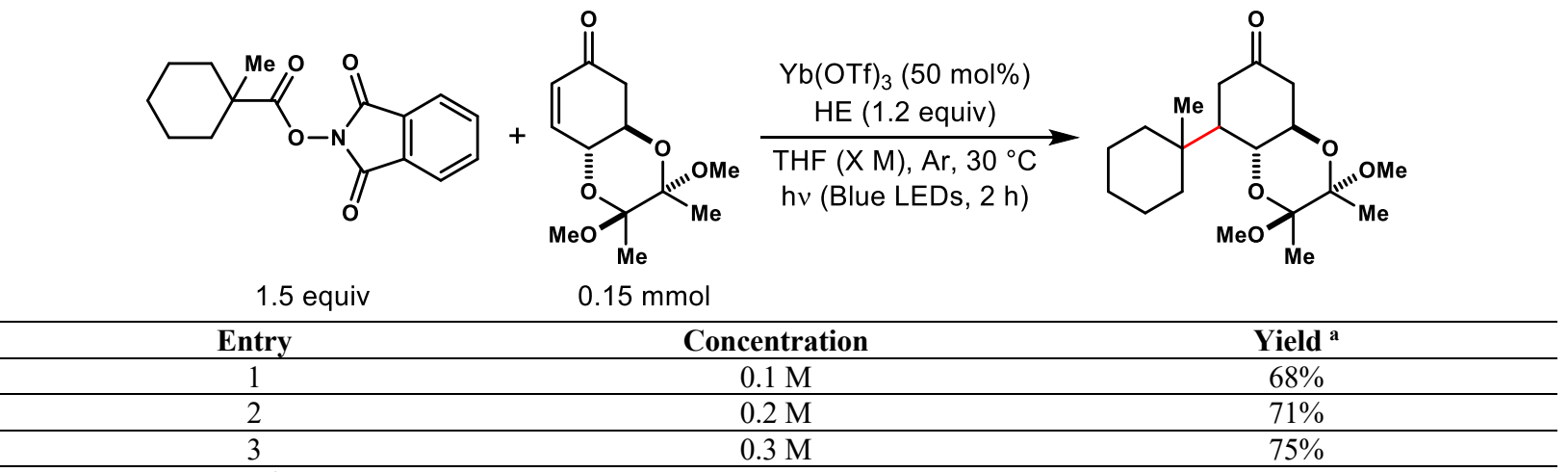

${ }^{\mathrm{a}}$ Yields determined by ${ }^{1} \mathrm{H}$ NMR spectroscopic analysis using 1,2-dibromo-4,5-methylenedioxybenzene as an external standard. 


\section{H. General Procedure for the Gd(OTf)3-Assisted Giese Coupling Reaction}

To an oven-dried 1-dram vial equipped with a magnetic stir bar was added the NHPI ester (0.225 mmol, 1.5 equiv), Hantzsch ester ( $46 \mathrm{mg}, 0.18 \mathrm{mmol}, 1.2$ equiv or $49 \mathrm{mg}, 0.195 \mathrm{mmol}, 1.3$ equiv), and $\mathrm{Gd}(\mathrm{OTf})_{3}$ (45 $\mathrm{mg}, 0.075 \mathrm{mmol}, 0.5$ equiv). Dry THF was added (0.5 or $\left.1.5 \mathrm{~mL}\right)$, followed by addition of the enone $(0.15 \mathrm{mmol}, 1.0$ equiv), and the reaction mixture was gently sparged with Ar for 10 min (Note: Volatile enones were added under Ar after sparging was complete). After sonication, the reaction mixture was vigorously stirred and irradiated with two Kessil Blue LED lamps $\left(\lambda_{\max }=456 \mathrm{~nm}\right)$ for $2 \mathrm{~h}$ while blowing a stream of air onto the 1-dram vial to maintain a reaction temperature of approximately $30^{\circ} \mathrm{C}$. Following irradiation, a solution of $1: 1$ $\mathrm{MeOH}: \mathrm{H}_{2} \mathrm{O}(500 \mu \mathrm{L})$ and DBU $(112 \mu \mathrm{L}, 0.75 \mathrm{mmol}, 5$ equiv $)$ was added and the reaction mixture was stirred vigorously at $\mathrm{rt}$ for an additional $30 \mathrm{~min}$. The reaction mixture was then transferred into a separatory funnel, diluted with $\mathrm{Et}_{2} \mathrm{O}(15 \mathrm{~mL})$, and washed with $1 \mathrm{~N} \mathrm{HCl}(\mathrm{aq})(15 \mathrm{~mL})$. The aqueous phase was extracted with an additional $15 \mathrm{~mL}$ of $\mathrm{Et}_{2} \mathrm{O}$, and the combined $\mathrm{Et}_{2} \mathrm{O}$ phases were dried with $\mathrm{MgSO}_{4}$ and concentrated (Note: If required, the majority of the Hantzsch ester and its resulting pyridine byproduct can be removed by washing the organic extracts with aq. $\mathrm{CuSO}_{4}(15 \mathrm{~mL})$ and $4 \mathrm{M} \mathrm{HCl}(\mathrm{aq})(3 \times 15 \mathrm{~mL})$. The crude mixture was then purified by flash column chromatography to furnish the pure 1,4-addition product. 


\section{Reaction Scope of the Gd(OTf) 3 -Assisted Giese Coupling Reaction}

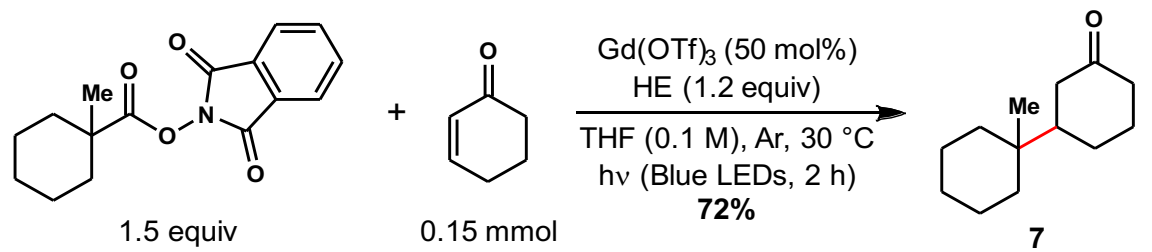

Ketone 7: According to the general procedure, cyclohexenone (15 $\mu \mathrm{L}, 0.15 \mathrm{mmol})$ was used as the acceptor. Purification of the product by flash column chromatography $\left(\mathrm{SiO}_{2}, 1: 0 \rightarrow 9: 1\right.$ Hex/EtOAc) provided ketone $7(21 \mathrm{mg}, 72 \%)$ as a colorless oil. $\mathrm{R}_{\mathrm{f}}=0.44$ (5:1 Hex/EtOAc, visualized with $\mathrm{KMnO}_{4}$ ). Characterization data acquired for the compound matched those previously reported. ${ }^{2}$

${ }^{1} \mathbf{H}$ NMR $\left(600 \mathrm{MHz}, \mathrm{CDCl}_{3}\right) \delta 2.45-2.40(\mathrm{~m}, 1 \mathrm{H}), 2.39-2.34(\mathrm{~m}, 1 \mathrm{H}), 2.24(\mathrm{ddt}, J=1.0,6.5,14.0$ $\mathrm{Hz}, 1 \mathrm{H}), 2.14-2.08(\mathrm{~m}, 1 \mathrm{H}), 2.06(\mathrm{dt}, J=1.0,13.4 \mathrm{~Hz}, 1 \mathrm{H}), 1.96-1.89$ (m, 1H), 1.67-1.61 (m, $1 \mathrm{H}), 1.59-1.20(\mathrm{~m}, 12 \mathrm{H}), 0.84(\mathrm{~s}, 3 \mathrm{H})$

${ }^{13} \mathrm{C}$ NMR $\left(150 \mathrm{MHz}, \mathrm{CDCl}_{3}\right) \delta$ 213.4, 47.9, 42.7, 41.6, 36.1, 35.7, 35.0, 26.4, 25.8, 25.1, 21.9, $21.8,19.8$
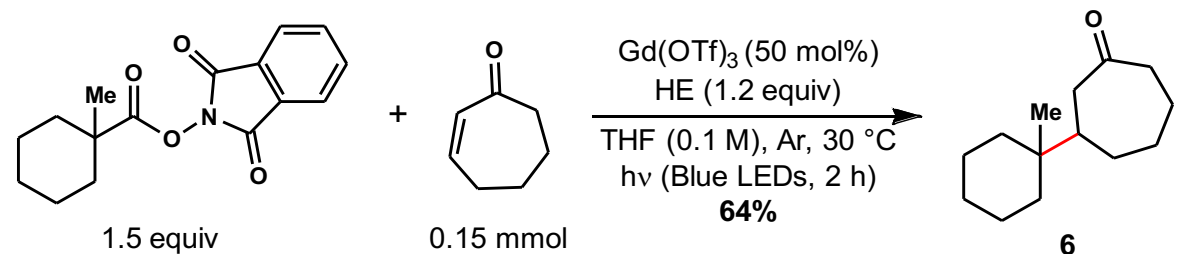

Ketone 6: According to the general procedure, cycloheptenone $(17 \mu \mathrm{L}, 0.15 \mathrm{mmol})$ was used as the acceptor. Purification of the product by flash column chromatography $\left(\mathrm{SiO}_{2}, 1: 0 \rightarrow 9: 1\right.$ Hex/EtOAc) provided ketone $6(20 \mathrm{mg}, 64 \%)$ as a colorless oil. $\mathrm{R}_{\mathrm{f}}=0.47$ (5:1 Hex/EtOAc, visualized with $\mathrm{KMnO}_{4}$ ).

${ }^{1} \mathbf{H}$ NMR $\left(600 \mathrm{MHz}, \mathrm{CDCl}_{3}\right) \delta 2.57-2.41(\mathrm{~m}, 3 \mathrm{H}), 2.33(\mathrm{dd}, J=11.4,14.1 \mathrm{~Hz}, 1 \mathrm{H}), 2.03-1.87(\mathrm{~m}$, $3 \mathrm{H}), 1.63-1.52(\mathrm{~m}, 3 \mathrm{H}), 1.52-1.45(\mathrm{~m}, 3 \mathrm{H}), 1.45-1.37(\mathrm{~m}, 2 \mathrm{H}), 1.36-1.32(\mathrm{~m}, 2 \mathrm{H}), 1.32-1.29(\mathrm{~m}$, $2 \mathrm{H}), 1.29-1.23(\mathrm{~m}, 1 \mathrm{H}), 1.11-1.02(\mathrm{~m}, 1 \mathrm{H}), 0.77(\mathrm{~s}, 3 \mathrm{H})$

${ }^{13} \mathrm{C}$ NMR $\left(150 \mathrm{MHz}, \mathrm{CDCl}_{3}\right) \delta 215.8,44.9,44.3,43.3,35.8,35.7,30.1,29.7,29.6,26.3,25.2$, $21.9,21.8,19.6$

IR (thin film, $\mathrm{cm}^{-1}$ ) 2923, 2856, 1701, 1446

HRMS (ESI-TOF) m/z: $[\mathrm{M}+\mathrm{Na}]^{+}$calcd for $\mathrm{C}_{14} \mathrm{H}_{24} \mathrm{ONa} 231.1725$, found 231.1723 


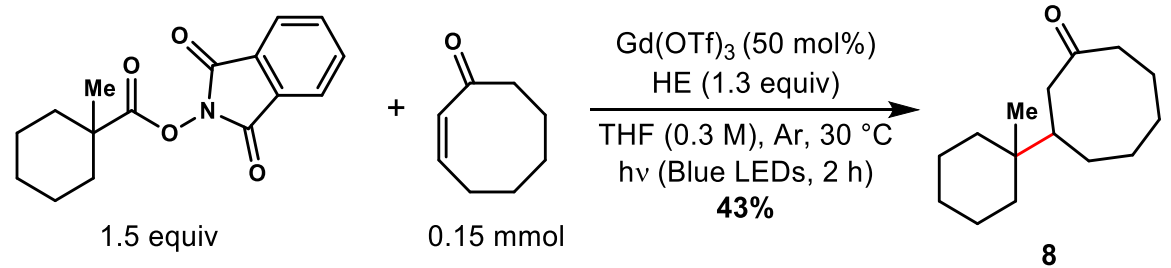

Ketone 8: According to the general procedure, cyclooctenone ${ }^{8}(18.6 \mathrm{mg}, 0.15 \mathrm{mmol})$ was used as the acceptor. Purification of the product by flash column chromatography $\left(\mathrm{SiO}_{2}, 1: 0 \rightarrow 9: 1\right.$ Hex/EtOAc) provided ketone $8(14.3 \mathrm{mg}, 43 \%)$ as a colorless oil. $\mathrm{R}_{\mathrm{f}}=0.5$ (4:1 Hex/EtOAc, visualized with $\mathrm{KMnO}_{4}$ ).

${ }^{1} \mathbf{H}$ NMR $\left(600 \mathrm{MHz}, \mathrm{CDCl}_{3}\right) \delta 2.52(\mathrm{dt}, J=4.5,12.7 \mathrm{~Hz}, 1 \mathrm{H}), 2.39-2.26(\mathrm{~m}, 3 \mathrm{H}), 1.99-1.81(\mathrm{~m}$, $3 \mathrm{H}), 1.80-1.73(\mathrm{~m}, 1 \mathrm{H}), 1.73-1.66(\mathrm{~m}, 1 \mathrm{H}), 1.57-1.28(\mathrm{~m}, 12 \mathrm{H}), 1.19-1.11(\mathrm{~m}, 1 \mathrm{H}), 0.78(\mathrm{~s}, 3 \mathrm{H})$

${ }^{13} \mathrm{C}$ NMR $\left(150 \mathrm{MHz}, \mathrm{CDCl}_{3}\right) \delta$ 218.6, 44.1, 43.2, 42.3, 36.8, 36.2, 35.4, 27.2, 27.1, 26.3, 25.9, $24.5,22.0,21.9,20.5$

IR (thin film, $\mathrm{cm}^{-1}$ ) 2921, 2856, 1697, 1465

HRMS (ESI-TOF) m/z: $[\mathrm{M}+\mathrm{Na}]^{+}$calcd for $\mathrm{C}_{15} \mathrm{H}_{26} \mathrm{ONa} 245.1881$, found 245.1877

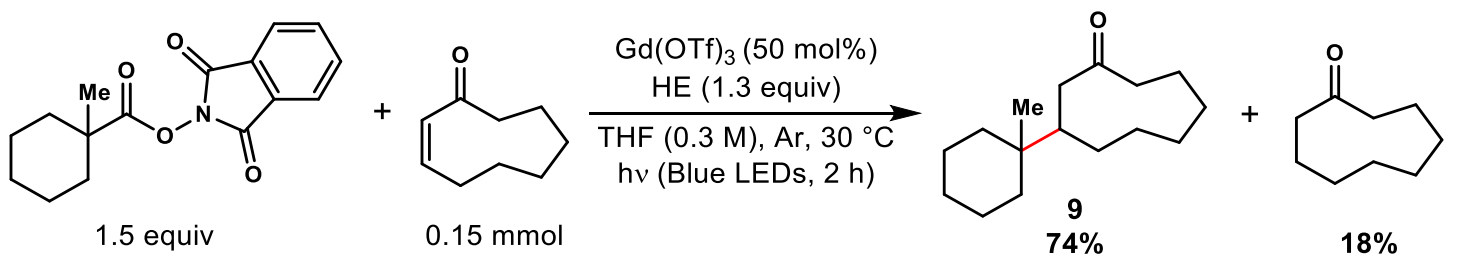

Ketone 9: According to the general procedure, $(Z)$-cyclononenone ${ }^{9}(20.7 \mathrm{mg}, 0.15 \mathrm{mmol})$ was used as the acceptor. Purification of the product by flash column chromatography $\left(\mathrm{SiO}_{2}, 1: 0 \rightarrow 9: 1\right.$ Hex/EtOAc) provided ketone $9(26.2 \mathrm{mg}, 74 \%)$ as a colorless oil, as well as reduced cyclononanone $(3.9 \mathrm{mg}, 18 \%)$. $\mathrm{R}_{\mathrm{f}}$ of $9=0.26\left(9: 1 \mathrm{Hex} /\right.$ EtOAc, visualized with $\left.\mathrm{KMnO}_{4}\right)$

${ }^{1} \mathbf{H}$ NMR $\left(600 \mathrm{MHz}, \mathrm{CDCl}_{3}\right) \delta 2.60(\mathrm{ddd}, J=3.0,10.9,13.7 \mathrm{~Hz}, 1 \mathrm{H}), 2.45-2.40(\mathrm{~m}, 1 \mathrm{H}), 2.39$ (dd, $J=2.2,12.2 \mathrm{~Hz}, 1 \mathrm{H}), 2.30($ app t, $J=11.5 \mathrm{~Hz}, 1 \mathrm{H}), 1.95-1.86(\mathrm{~m}, 2 \mathrm{H}), 1.80-1.71(\mathrm{~m}, 1 \mathrm{H})$, $1.67-1.54(\mathrm{~m}, 5 \mathrm{H}), 1.53-1.46(\mathrm{~m}, 3 \mathrm{H}), 1.45-1.37(\mathrm{~m}, 4 \mathrm{H}), 1.36-1.32(\mathrm{~m}, 4 \mathrm{H}), 1.31-1.28(\mathrm{~m}, 1 \mathrm{H})$, $1.25-1.20(\mathrm{~m}, 1 \mathrm{H}), 0.78(\mathrm{~s}, 3 \mathrm{H})$

${ }^{13} \mathbf{C}$ NMR $\left(150 \mathrm{MHz}, \mathrm{CDCl}_{3}\right) \delta 218.4,45.9,43.3,41.8,36.5,36.2,35.7,26.6,26.4,26.3,25.7$, $25.1,24.1,21.99,21.98,20.1$

IR (thin film, $\mathrm{cm}^{-1}$ ) 2924, 2853, 1698, 1465

HRMS (ESI-TOF) m/z: [M+Na] ${ }^{+}$calcd for $\mathrm{C}_{16} \mathrm{H}_{28} \mathrm{ONa} 259.2038$, found 259.2048 


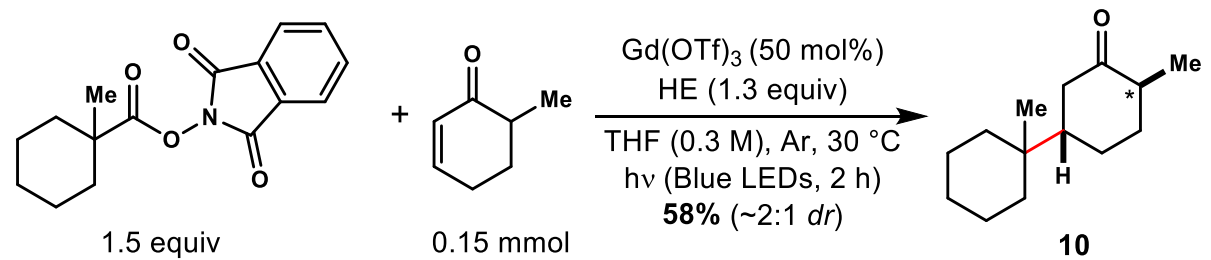

Ketone 10: According to the general procedure, 6-methylcyclohexenone ${ }^{10}(16.5 \mathrm{mg}, 0.15 \mathrm{mmol})$ was used as the acceptor. Purification of the product by flash column chromatography $\left(\mathrm{SiO}_{2}\right.$, $1: 0 \rightarrow 9: 1 \mathrm{Hex} /$ EtOAc) provided ketone $10(18.1 \mathrm{mg}, 58 \%)$ as an inseparable 2:1 mixture of diastereomers and as a colorless oil. $\mathrm{R}_{\mathrm{f}}=0.34(9: 1 \mathrm{Hex} / \mathrm{EtOAc})$.

${ }^{1} \mathbf{H}$ NMR $\left(600 \mathrm{MHz}, \mathrm{CDCl}_{3}\right) \delta 2.53-2.45(\mathrm{~m}, 0.3 \mathrm{H}), 2.42(\mathrm{ddd}, J=2.6,3.4,12.9 \mathrm{~Hz}, 0.7 \mathrm{H}), 2.35-$ $2.38(\mathrm{~m}, 0.7 \mathrm{H}), 2.26-2.19(\mathrm{~m}, 0.3 \mathrm{H}), 2.14-2.08(\mathrm{~m}, 0.7 \mathrm{H}), 2.08-2.02(\mathrm{~m}, 0.7 \mathrm{H}), 1.91-1.86(\mathrm{~m}$, $1 \mathrm{H}), 1.82-1.77(\mathrm{~m}, 0.3 \mathrm{H}), 1.76-1.71(\mathrm{~m}, 0.3 \mathrm{H}), 1.69-1.59(\mathrm{~m}, 1 \mathrm{H}), 1.54-1.32(\mathrm{~m}, 7 \mathrm{H}), 1.31-1.19$ $(\mathrm{m}, 5 \mathrm{H}), 1.14(\mathrm{~d}, J=7.2 \mathrm{~Hz}, 0.9 \mathrm{H}), 1.00(\mathrm{~d}, J=6.5 \mathrm{~Hz}, 2.1 \mathrm{H}), 0.83(\mathrm{~s}, 3 \mathrm{H})$

${ }^{13} \mathrm{C}$ NMR (150 MHz, $\left.\mathrm{CDCl}_{3}\right) \delta$ (major) 214.5, 49.0, 45.1, 42.7, 36.1, 35.8, 35.2, 35.0, 26.4, 25.6, 21.9, 21.8, 19.84, 14.4 (minor, not all observed) 217.4, 46.8, 43.4, 38.8, 36.0, 35.7, 31.4, 29.7, $19.9,19.78,16.7$

IR (thin film, $\mathrm{cm}^{-1}$ ) 2924, 2853, 1711, 1456, 1377

HRMS (ESI-TOF) m/z: [M+H] $]^{+}$calcd for $\mathrm{C}_{14} \mathrm{H}_{25} \mathrm{O} 209.1905$, observed 208.1898

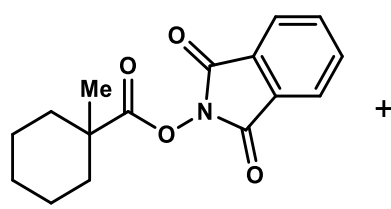

1.5 equiv

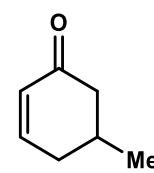

$0.15 \mathrm{mmo}$

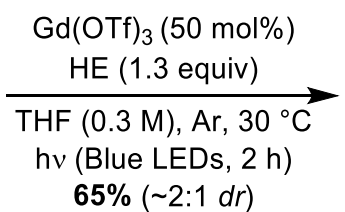

$65 \%(\sim 2: 1 d r)$

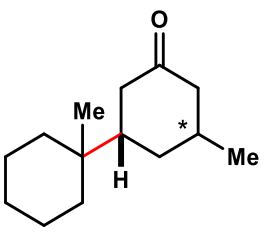

11

Ketone 11: According to the general procedure, 5-methylcyclohexenone ${ }^{11}$ (16.5 $\left.\mathrm{mg}, 0.15 \mathrm{mmol}\right)$ was used as the acceptor. Purification of the product by flash column chromatography $\left(\mathrm{SiO}_{2}\right.$, $1: 0 \rightarrow 9: 1 \mathrm{Hex} /$ EtOAc) provided ketone $11(20.2 \mathrm{mg}, 65 \%)$ as an inseparable 2:1 mixture of diastereomers and as a colorless oil. $\mathrm{R}_{\mathrm{f}}=0.29(9: 1 \mathrm{Hex} / \mathrm{EtOAc})$.

${ }^{1} \mathbf{H}$ NMR $\left(600 \mathrm{MHz}, \mathrm{CDCl}_{3}\right) \delta$ 2.48-2.32 (m, 3H), 2.14-1.85 (m, 4H), 1.80-1.70 (m, 1H), 1.68 $1.58(\mathrm{~m}, 2 \mathrm{H}), 1.50-1.31(\mathrm{~m}, 5 \mathrm{H}), 1.29-1.21(\mathrm{~m}, 3 \mathrm{H}), 1.04(\mathrm{~d}, J=6.4 \mathrm{~Hz}, 1.25 \mathrm{H}), 0.95(\mathrm{~d}, J=7.0$ $\mathrm{Hz}, 1.75 \mathrm{H}), 0.83(\mathrm{~s}, 3 \mathrm{H})$

${ }^{13}$ C NMR $\left(150 \mathrm{MHz}, \mathrm{CDCl}_{3}\right) \delta 213.8,213.1,49.9,47.7,46.6,42.3,41.8,41.3,36.14,36.12,35.9$, $34.9,34.7,34.2,33.3,30.9,29.6,26.4,22.7,21.9,21.8,21.77,19.8,19.4$

IR (thin film, $\mathrm{cm}^{-1}$ ) 2924, 2857, 1711, 1458

HRMS (ESI-TOF) m/z: [M+H] $]^{+}$calcd for $\mathrm{C}_{14} \mathrm{H}_{25} \mathrm{O} 209.1905$, found 208.1911 


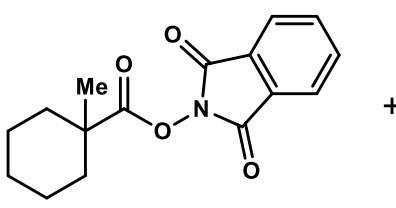

1.5 equiv

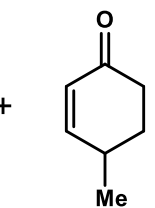

$0.15 \mathrm{mmol}$

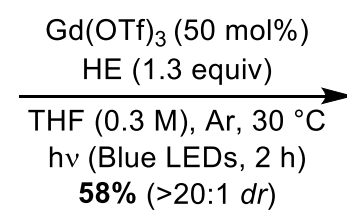

$58 \%(>20: 1 d r)$

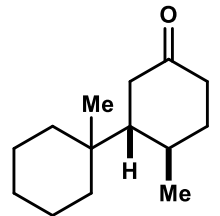

12

Ketone 12: According to the general procedure, 4-methylcyclohexenone ${ }^{12}(16.5 \mathrm{mg}, 0.15 \mathrm{mmol})$ was used as the acceptor. Purification of the product by flash column chromatography $\left(\mathrm{SiO}_{2}\right.$, $1: 0 \rightarrow 9: 1 \mathrm{Hex} /$ EtOAc) provided ketone $12(18.0 \mathrm{mg}, 58 \%)$ as a colorless oil. $\mathrm{R}_{\mathrm{f}}=0.4(9: 1$ $\mathrm{Hex} / \mathrm{EtOAc})$.

${ }^{1} \mathbf{H}$ NMR $\left(600 \mathrm{MHz}, \mathrm{CDCl}_{3}\right) \delta 2.37-2.27(\mathrm{~m}, 3 \mathrm{H}), 2.23(\mathrm{dt}, J=5.2,18.8 \mathrm{~Hz}, 1 \mathrm{H}), 2.04-1.93(\mathrm{~m}$, $2 \mathrm{H}), 1.62(\mathrm{dq}, J=4.3,13.7 \mathrm{~Hz}, 1 \mathrm{H}), 1.56-1.35(\mathrm{~m}, 7 \mathrm{H}), 1.33-1.27(\mathrm{~m}, 2 \mathrm{H}), 1.26-1.18(\mathrm{~m}, 2 \mathrm{H})$, $1.03(\mathrm{~d}, J=6.9 \mathrm{~Hz}, 3 \mathrm{H}), 0.86(\mathrm{~s}, 3 \mathrm{H})$

${ }^{13} \mathrm{C}$ NMR $\left(150 \mathrm{MHz}, \mathrm{CDCl}_{3}\right) \delta 216.2,49.4,39.2,36.8,36.0,35.8,35.4,28.8,27.1,26.4,23.2$, $22.0,21.8,19.7$

IR (thin film, $\mathrm{cm}^{-1}$ ) 2926, 2861, 1715, 1465, 1379

HRMS (ESI-TOF) m/z: $[\mathrm{M}+\mathrm{Na}]^{+}$calcd for $\mathrm{C}_{14} \mathrm{H}_{24} \mathrm{ONa} 231.1725$, found 231.1739

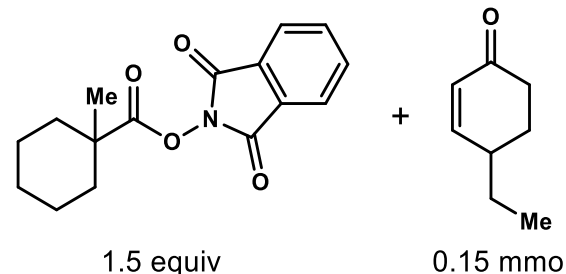

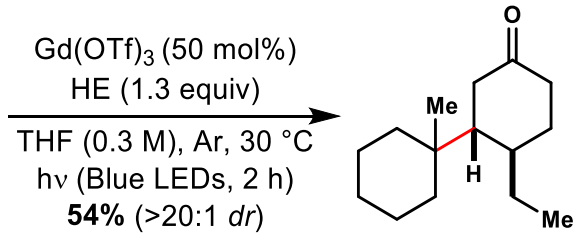

13

Ketone 13: According to the general procedure, 4-ethylcyclohexenone ${ }^{13}$ (18.6 $\left.\mathrm{mg}, 0.15 \mathrm{mmol}\right)$ was used as the acceptor. Purification of the product by flash column chromatography $\left(\mathrm{SiO}_{2}\right.$, $1: 0 \rightarrow 9: 1 \mathrm{Hex} /$ EtOAc) provided ketone $13(18.1 \mathrm{mg}, 54 \%)$ as a colorless oil. $\mathrm{R}_{\mathrm{f}}=0.42(9: 1$ $\mathrm{Hex} / \mathrm{EtOAc})$.

${ }^{1} \mathbf{H}$ NMR $\left(600 \mathrm{MHz}, \mathrm{CDCl}_{3}\right) \delta 2.36(\mathrm{dd}, J=9.7,14.1 \mathrm{~Hz}, 1 \mathrm{H}), 2.30-2.16(\mathrm{~m}, 3 \mathrm{H}), 1.94-1.86(\mathrm{~m}$, $1 \mathrm{H}), 1.77(\mathrm{dq}, J=4.7,14.1 \mathrm{~Hz}, 1 \mathrm{H}), 1.74-1.68(\mathrm{~m}, 1 \mathrm{H}), 1.56-1.44(\mathrm{~m}, 4 \mathrm{H}), 1.42-1.24(\mathrm{~m}, 7 \mathrm{H})$, $1.24-1.15(\mathrm{~m}, 2 \mathrm{H}), 0.89(\mathrm{t}, J=7.4 \mathrm{~Hz}, 3 \mathrm{H}), 0.86(\mathrm{~s}, 3 \mathrm{H})$

${ }^{13} \mathrm{C}$ NMR $\left(150 \mathrm{MHz}, \mathrm{CDCl}_{3}\right) \delta 216.4,48.8,39.1,36.8,35.8,35.3,35.2,33.7,28.7,26.4,23.9$, $22.0,21.8,19.7,12.1$

IR (thin film, $\mathrm{cm}^{-1}$ ) 2923, 2860, 1714, 1460, 1379

HRMS (ESI-TOF) m/z: $[\mathrm{M}+\mathrm{H}]^{+}$calcd for $\mathrm{C}_{15} \mathrm{H}_{27} \mathrm{O}$ 223.2062, found 223.2058 

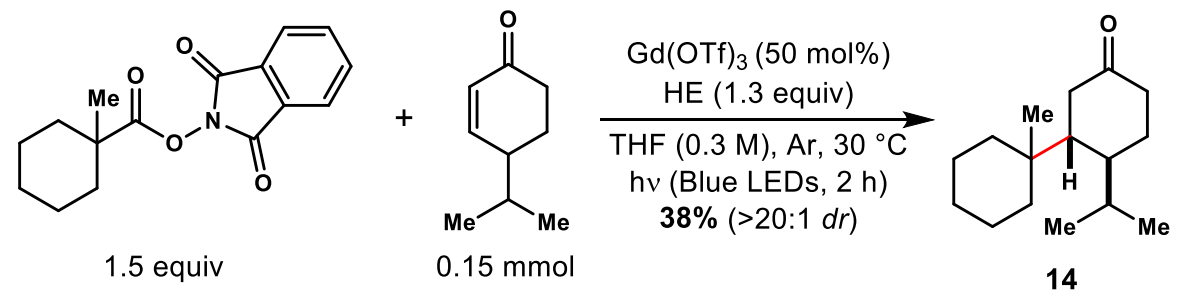

Ketone 14: According to the general procedure, cryptone ${ }^{14}(21 \mathrm{mg}, 0.15 \mathrm{mmol})$ was used as the acceptor. Purification of the product by flash column chromatography $\left(\mathrm{SiO}_{2}, 1: 0 \rightarrow 9: 1\right.$ Hex/EtOAc) provided ketone $14(13 \mathrm{mg}, 38 \%)$ as a colorless oil. $\mathrm{R}_{\mathrm{f}}=0.38$ (9:1 Hex/EtOAc).

${ }^{1} \mathbf{H}$ NMR $\left(600 \mathrm{MHz}, \mathrm{CDCl}_{3}\right) \delta 2.44(\mathrm{dd}, J=5.6,14.8 \mathrm{~Hz}, 1 \mathrm{H}), 2.33-2.27(\mathrm{~m}, 1 \mathrm{H}), 2.26(\mathrm{dd}, J=$ 7.9, $14.8 \mathrm{~Hz}, 1 \mathrm{H}), 2.16$ (ddd, $J=6.0,7.5,18.7 \mathrm{~Hz}, 1 \mathrm{H}), 1.87-1.80(\mathrm{~m}, 1 \mathrm{H}), 1.79-1.73(\mathrm{~m}, 1 \mathrm{H})$, 1.73-1.67 (m, 1H), 1.66-1.53 (m, 3H), 1.53-1.45 (m, 2H), 1.44-1.31 (m, 4H), 1.25-1.11 (m, 3H), $0.96(\mathrm{~d}, J=6.5 \mathrm{~Hz}, 3 \mathrm{H}), 0.90(\mathrm{~d}, J=6.5 \mathrm{~Hz}, 3 \mathrm{H}), 0.84(\mathrm{~s}, 3 \mathrm{H})$

${ }^{13}$ C NMR $\left(125 \mathrm{MHz}, \mathrm{CDCl}_{3}\right) \delta 216.4,46.9,39.5,38.4,37.3,37.1,35.9,35.3,31.8,26.4,22.04$, $21.95,21.8,21.3,19.8,18.4$

IR (thin film, $\mathrm{cm}^{-1}$ ) 2926, 2863, 1714, 1464, 1385

HRMS (ESI-TOF) m/z: [M+Na] ${ }^{+}$calcd for $\mathrm{C}_{16} \mathrm{H}_{28} \mathrm{ONa} 259.2038$, found 259.2038

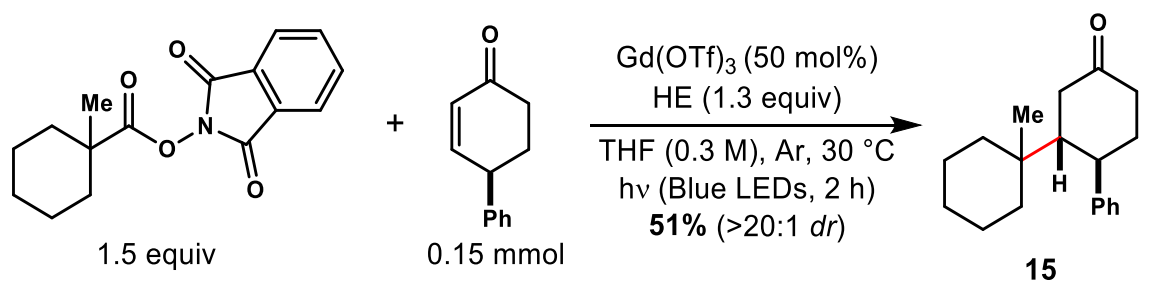

Ketone 15: According to the general procedure, 4-phenylcyclohexenone $\mathrm{e}^{15}(25.8 \mathrm{mg}, 0.15 \mathrm{mmol})$ was used as the acceptor. Purification of the product by flash column chromatography $\left(\mathrm{SiO}_{2}\right.$, $1: 0 \rightarrow 9: 1 \mathrm{Hex} /$ EtOAc) provided ketone $15(20.6 \mathrm{mg}, 51 \%)$ as a colorless oil. $\mathrm{R}_{\mathrm{f}}=0.4(9: 1$ $\mathrm{Hex} /$ EtOAc, visualized with $\mathrm{KMNO}_{4}$ ).

${ }^{1} \mathbf{H}$ NMR $\left(600 \mathrm{MHz}, \mathrm{CDCl}_{3}\right) \delta 7.28(\mathrm{t}, J=7.6 \mathrm{~Hz}, 2 \mathrm{H}), 7.23-7.16(\mathrm{~m}, 3 \mathrm{H}), 3.05-2.99(\mathrm{~m}, 1 \mathrm{H})$, 2.57 (dd, $J=6.2,14.5 \mathrm{~Hz}, 1 \mathrm{H}), 2.44(\mathrm{dd}, J=9.5,14.5 \mathrm{~Hz}, 1 \mathrm{H}), 2.39-2.25(\mathrm{~m}, 3 \mathrm{H}), 2.14-2.05(\mathrm{~m}$, $1 \mathrm{H}), 1.91-1.82(\mathrm{~m}, 1 \mathrm{H}), 1.44-1.30(\mathrm{~m}, 3 \mathrm{H}), 1.27-1.11(\mathrm{~m}, 5 \mathrm{H}), 1.09-1.02(\mathrm{~m}, 1 \mathrm{H}), 1.96-1.88(\mathrm{~m}$, $1 \mathrm{H}), 0.87(\mathrm{~s}, 3 \mathrm{H})$

${ }^{13} \mathrm{C}$ NMR $\left(150 \mathrm{MHz}, \mathrm{CDCl}_{3}\right) \delta 214.9,147.7,128.6,127.7,126.1,47.2,41.9,40.4,37.9,37.4$, $36.7,36.1,32.7,26.1,21.8,21.7,20.0$

IR (thin film, $\mathrm{cm}^{-1}$ ) 2923, 2856, 1711, 1493, 1451, 1380

HRMS (ESI-TOF) m/z: $[\mathrm{M}+\mathrm{H}]^{+}$calcd for $\mathrm{C}_{19} \mathrm{H}_{27} \mathrm{O} 271.2062$, found 271.2072 


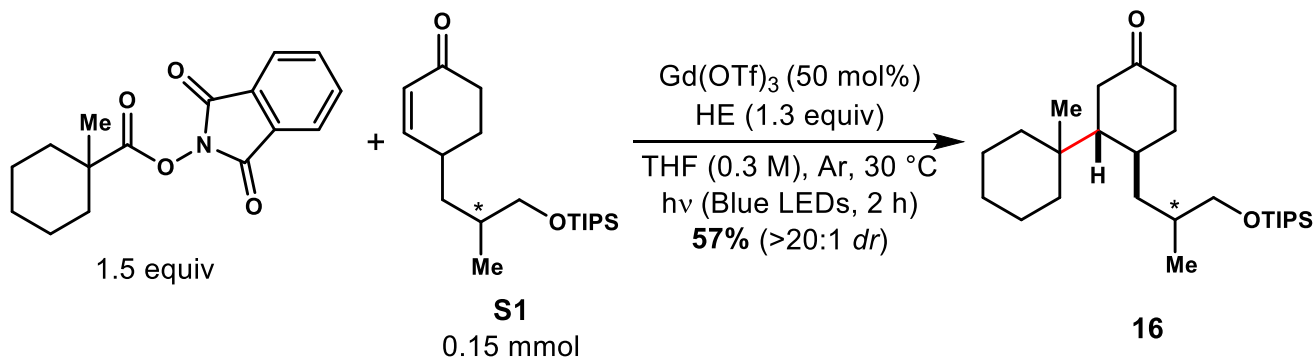

Ketone 16: According to the general procedure, enone S1 (47 mg, $0.15 \mathrm{mmol}$ ) was used as the acceptor. Purification of the product by flash column chromatography $\left(\mathrm{SiO}_{2}, 1: 0 \rightarrow 9: 1\right.$ Hex/EtOAc) provided ketone 16 (36.2 mg, 57\%), a mixture of stereoisomers, as a colorless oil. $\mathrm{R}_{\mathrm{f}}$ $=0.54(9: 1 \mathrm{Hex} /$ EtOAc $)$.

${ }^{1} \mathbf{H}$ NMR $\left(600 \mathrm{MHz}, \mathrm{CDCl}_{3}\right) \delta 3.59-3.52(\mathrm{~m}, 1 \mathrm{H}), 3.46(\mathrm{~d}, J=6.2 \mathrm{~Hz}, 1 \mathrm{H}), 2.36(\mathrm{dd}, J=8.7,13.9$ $\mathrm{Hz}, 1 \mathrm{H}), 2.32-2.16(\mathrm{~m}, 3 \mathrm{H}), 2.08-1.85(\mathrm{~m}, 2 \mathrm{H}), 1.58-1.35(\mathrm{~m}, 9 \mathrm{H}), 1.33-1.26(\mathrm{~m}, 2 \mathrm{H}), 1.25-1.16$ $(\mathrm{m}, 2 \mathrm{H}), 1.11-1.03(\mathrm{~m}, 21 \mathrm{H}), 0.94(\mathrm{~d}, J=4.0 \mathrm{~Hz}, 1.5 \mathrm{H}), 0.92(\mathrm{~d}, J=3.9 \mathrm{~Hz}, 1.5 \mathrm{H}), 0.88(\mathrm{~s}, 1.5 \mathrm{H})$, $0.87(\mathrm{~s}, 1.5 \mathrm{H})$

${ }^{13} \mathrm{C}$ NMR $\left(150 \mathrm{MHz}, \mathrm{CDCl}_{3}\right) \delta 216.24,216.20,69.3,67.8,49.4,49.3,40.3,39.3,39.01,38.95$, $37.1,36.8,35.9,35.86,35.4,35.37,35.36,35.2,33.6,33.4,29.3,28.60,26.40,26.36,25.2$, 23.7, $22.01,21.98,21.83,21.81,19.8,19.7,18.5,18.10,18.09,15.9,12.04,12.01$

IR (thin film, $\mathrm{cm}^{-1}$ ) 2927, 2864, 1716, 1462, 1381, 1243, 1100, 1066, 882

HRMS (ESI-TOF) m/z: $[\mathrm{M}+\mathrm{Na}]^{+}$calcd for $\mathrm{C}_{26} \mathrm{H}_{50} \mathrm{O}_{2} \mathrm{SiNa} 445.3478$, found 445.3475

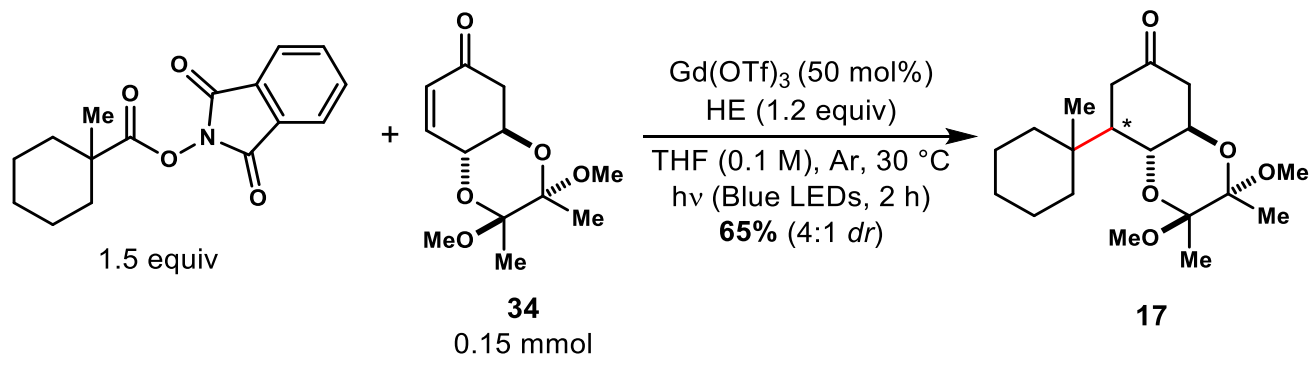

Ketone 17: According to the general procedure, enone $34^{16}(36 \mathrm{mg}, 0.15 \mathrm{mmol})$ was used as the acceptor. Purification of the product by flash column chromatography $\left(\mathrm{SiO}_{2}, 1: 0 \rightarrow 85: 15\right.$ Hex/EtOAc) provided ketone 17 (33 mg, 65\%), a 4:1 mixture of stereoisomers, as a colorless oil. $\mathrm{R}_{\mathrm{f}}=0.38(4: 1 \mathrm{Hex} / \mathrm{EtOAc})$

${ }^{1} \mathbf{H}$ NMR $\left(600 \mathrm{MHz}, \mathrm{CDCl}_{3}\right) \delta 4.38(\mathrm{dt}, J=6.4,11.2 \mathrm{~Hz}, 0.2 \mathrm{H}), 4.17(\mathrm{dd}, J=5.7,10.9 \mathrm{~Hz}, 0.2 \mathrm{H})$, 4.09 (app t, $J=9.6 \mathrm{~Hz}, 0.8 \mathrm{H}), 3.92-3.85(\mathrm{~m}, 0.8 \mathrm{H}), 3.39(\mathrm{~s}, 2.4 \mathrm{H}), 3.28(\mathrm{~s}, 0.6 \mathrm{H}), 3.24(\mathrm{~s}, 0.6 \mathrm{H})$, $3.23(\mathrm{~s}, 2.4 \mathrm{H}), 2.70-2.62(\mathrm{~m}, 0.4 \mathrm{H}), 2.57-2.41(\mathrm{~m}, 2.4 \mathrm{H}), 2.33(\mathrm{dd}, J=7.1,15.7 \mathrm{~Hz}, 0.2 \mathrm{H}), 2.26-$ $2.21(\mathrm{~m}, 0.2 \mathrm{H}), 2.14(\mathrm{dd}, J=10.7,15.2 \mathrm{~Hz}, 0.8 \mathrm{H}), 2.07-2.00(\mathrm{~m}, 0.4 \mathrm{H}), 1.96-1.89(\mathrm{~m}, 0.8 \mathrm{H}), 1.73-$ 
$1.64(\mathrm{~m}, 0.8 \mathrm{H}), 1.64-1.50(\mathrm{~m}, 3 \mathrm{H}), 1.49-1.34(\mathrm{~m}, 5 \mathrm{H}), 1.31(\mathrm{~s}, 2.4 \mathrm{H}), 1.30(\mathrm{~s}, 2.4 \mathrm{H}), 1.28(\mathrm{~s}, 0.6 \mathrm{H})$, $1.27-1.23(\mathrm{~s}, 1 \mathrm{H}), 1.22(\mathrm{~s}, 0.6 \mathrm{H}), 0.95(\mathrm{~s}, 0.6 \mathrm{H}), 0.94(\mathrm{~s}, 2.4 \mathrm{H})$

${ }^{13} \mathrm{C}$ NMR (150 MHz, $\mathrm{CDCl}_{3}$ ) $\delta$ (Major) 208.6, 99.7, 99.1, 71.8, 68.1, 49.6, 48.0, 44.2, 43.4, 40.6, 37.1, 36.7, 36.0, 26.2, 22.0, 21.9, 20.3, 17.9, 17.8 (Minor) 209.2, 99.5, 98.9, 74.2, 64.9, 48.0, 47.9, $44.9,42.4,38.0,37.7,37.2,35.4,25.7,23.6,23.2,22.1,17.8,17.6$

IR (thin film, $\mathrm{cm}^{-1}$ ) 2923, 2859, 1720, 1461, 1376, 1212, 1116, 1079, 1053, 1037, 936

HRMS (ESI-TOF) m/z: [M+Na] ${ }^{+}$calcd for $\mathrm{C}_{19} \mathrm{H}_{32} \mathrm{O}_{5} \mathrm{Na} 363.2148$, found 363.2157

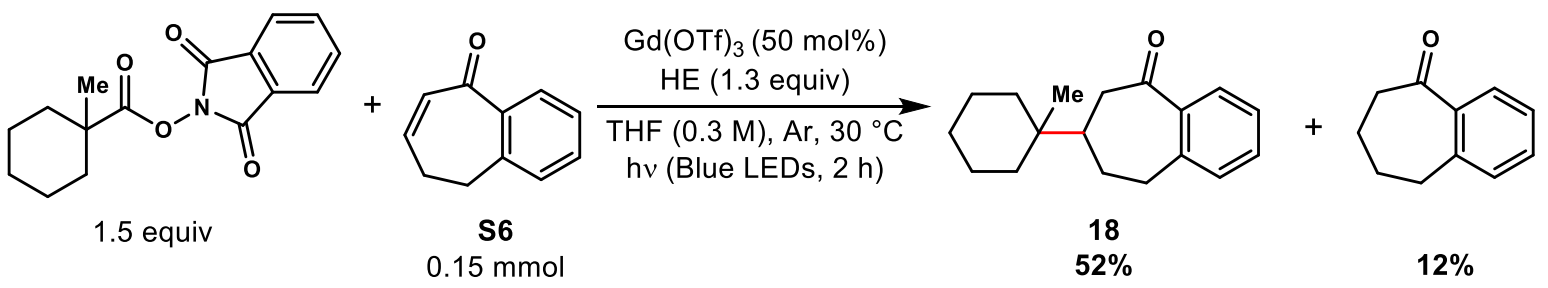

Ketone 18: According to the general procedure, enone $\mathbf{S 6}{ }^{15}(24 \mathrm{mg}, 0.15 \mathrm{mmol})$ was used as the acceptor. Purification of the product by flash column chromatography $\left(\mathrm{SiO}_{2}, 1: 0 \rightarrow 85: 15\right.$ Hex/EtOAc) provided ketone $\mathbf{1 8}(20.2 \mathrm{mg}, 52 \%)$ as a colorless oil, as well as the reduction product, 1-benzosuberone (3.0 mg, 12\%). $\mathrm{R}_{\mathrm{f}} \mathbf{1 8}=0.5(9: 1 \mathrm{Hex} /$ EtOAc).

${ }^{1} \mathbf{H}$ NMR $\left(600 \mathrm{MHz}, \mathrm{CDCl}_{3}\right) \delta 7.77(\mathrm{~d}, J=7.6 \mathrm{~Hz}, 1 \mathrm{H}), 7.43(\mathrm{t}, J=7.4 \mathrm{~Hz}, 1 \mathrm{H}), 7.31(\mathrm{t}, J=7.5$ $\mathrm{Hz}, 1 \mathrm{H}), 7.19(\mathrm{~d}, J=7.5 \mathrm{~Hz}, 1 \mathrm{H}), 2.99(\mathrm{dt}, J=5.8,13.4 \mathrm{~Hz}, 1 \mathrm{H}), 2.85$ (ddd, $J=3.3,4.9,14.5 \mathrm{~Hz}$, $1 \mathrm{H}), 2.78(\mathrm{~d}, J=17.1 \mathrm{~Hz}, 1 \mathrm{H}), 2.54(\mathrm{dd}, J=11.3,17.1 \mathrm{~Hz}, 1 \mathrm{H}), 1.87-1.79(\mathrm{~m}, 1 \mathrm{H}), 1.77-1.69$ (m, $1 \mathrm{H}), 1.65-1.59(\mathrm{~m}, 1 \mathrm{H}), 1.51-1.45(\mathrm{~m}, 1 \mathrm{H}), 1.43-1.34(\mathrm{~m}, 4 \mathrm{H}), 1.30-1.22(\mathrm{~m}, 4 \mathrm{H}), 1.19-1.12(\mathrm{~m}$, $1 \mathrm{H}), 0.83(\mathrm{~s}, 3 \mathrm{H})$

${ }^{13}$ C NMR $\left(150 \mathrm{MHz}, \mathrm{CDCl}_{3}\right) \delta 205.6,141.4,137.9,132.6,129.6,128.6,126.8,42.6,40.5,36.0$, $35.5,32.2,29.7,26.3,26.0,21.87,21.84,19.7$

IR (thin film) 2924, 2857, 1672, 1598, 1448, 1380, 1289, 1246

HRMS (ESI-TOF) m/z: [M+H] $]^{+}$calcd for $\mathrm{C}_{18} \mathrm{H}_{25} \mathrm{O} 257.1906$, found 257.1895

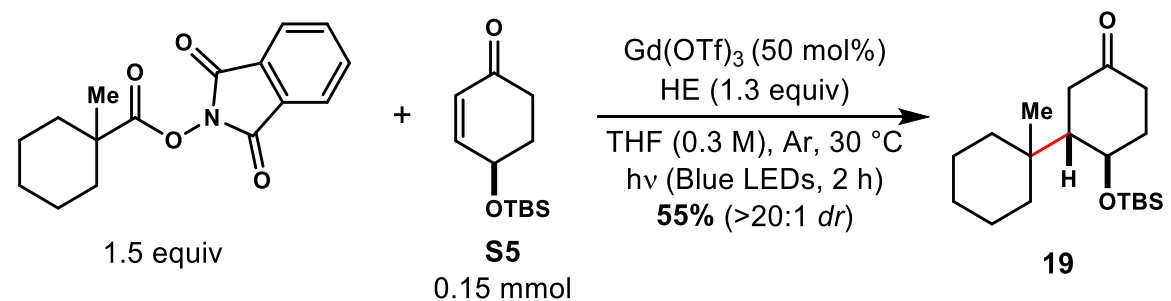

Ketone 19: According to the general procedure, enone $\mathbf{S 5}^{\mathbf{1 7}}$ (33.9 $\left.\mathrm{mg}, 0.15 \mathrm{mmol}\right)$ was used as the acceptor. Purification of the product by flash column chromatography $\left(\mathrm{SiO}_{2}, 1: 0 \rightarrow 9: 1\right.$ 
Hex/EtOAc) provided ketone $19(27 \mathrm{mg}, 55 \%)$ as a colorless oil. $\mathrm{R}_{\mathrm{f}}=0.51$ (9:1 Hex/EtOAc, visualized with $\mathrm{KMNO}_{4}$ ).

${ }^{1} \mathbf{H}$ NMR $\left(600 \mathrm{MHz}, \mathrm{CDCl}_{3}\right) \delta 4.17$ (app q, $\left.J=3.0 \mathrm{~Hz}, 1 \mathrm{H}\right), 2.55$ (ddd, $J=7.8,11.0,17.8 \mathrm{~Hz}$, $1 \mathrm{H}), 2.48(\mathrm{dd}, J=6.8,14.7 \mathrm{~Hz}, 1 \mathrm{H}), 2.27-2.16(\mathrm{~m}, 2 \mathrm{H}), 1.98-1.89(\mathrm{~m}, 3 \mathrm{H}), 1.53-1.33(\mathrm{~m}, 7 \mathrm{H})$, $1.29-1.23(\mathrm{~m}, 3 \mathrm{H}), 0.89(\mathrm{~s}, 9 \mathrm{H}), 0.85(\mathrm{~s}, 3 \mathrm{H}), 0.09(\mathrm{~s}, 6 \mathrm{H})$

${ }^{13}$ C NMR $\left(150 \mathrm{MHz}, \mathrm{CDCl}_{3}\right) \delta 214.3,66.7,52.1,38.3,36.3,36.1,35.4,34.6,30.5,26.2,25.8$, $21.9,21.6,21.0,17.9,-4.2,-4.5$

IR (thin film, $\mathrm{cm}^{-1}$ ) 2926, 2855, 1716, 1461, 1068, 1004, 834

HRMS (ESI-TOF) m/z: [M+Na] $]^{+}$calcd for $\mathrm{C}_{19} \mathrm{H}_{36} \mathrm{O}_{2} \mathrm{SiNa} 347.2382$, found 347.2378

Optical Rotation $[\alpha]^{21.5} \mathrm{D}-52.0,[\alpha]^{21.5}{ }_{577}-55.5,[\alpha]^{21.6}{ }_{546}-65.9,[\alpha]^{21.6}{ }_{435}-123.9\left(c=1.0, \mathrm{CHCl}_{3}\right)$

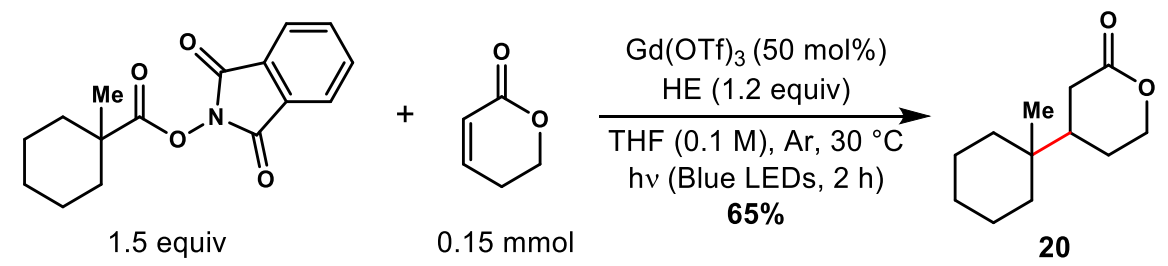

Lactone 20: According to the general procedure, 5,6-dihydro- $2 H$-pyran-2-one ( $13 \mu \mathrm{L}, 0.15 \mathrm{mmol})$ was used as the acceptor. Purification of the product by flash column chromatography $\left(\mathrm{SiO}_{2}\right.$, $1: 0 \rightarrow 85: 15 \mathrm{Hex} / \mathrm{EtOAc})$ provided lactone $20(18 \mathrm{mg}, 65 \%)$ as a colorless oil. $\mathrm{R}_{\mathrm{f}}=0.17(4: 1$ $\mathrm{Hex} / \mathrm{EtOAc}$, visualized with $\mathrm{KMnO}_{4}$ ).

${ }^{1} \mathbf{H}$ NMR $\left(600 \mathrm{MHz}, \mathrm{CDCl}_{3}\right) \delta 4.38(\mathrm{dt}, J=4.3,11.2 \mathrm{~Hz}, 1 \mathrm{H}), 4.2(\mathrm{dt}, J=3.3,10.8 \mathrm{~Hz}, 1 \mathrm{H}), 2.62-$ $2.54(\mathrm{~m}, 1 \mathrm{H}), 2.30(\mathrm{dd}, J=10.8,17.0 \mathrm{~Hz}, 1 \mathrm{H}), 1.93-1.83(\mathrm{~m}, 2 \mathrm{H}), 1.64-1.38(\mathrm{~m}, 6 \mathrm{H}), 1.37-1.18$ $(\mathrm{m}, 5 \mathrm{H}), 0.83(\mathrm{~s}, 3 \mathrm{H})$

${ }^{13}$ C NMR $\left(150 \mathrm{MHz}, \mathrm{CDCl}_{3}\right) \delta 172.8,68.8,40.4,35.3,35.0,34.7,31.0,26.2,23.5,21.6,21.5$, 19.2

IR (thin film, $\mathrm{cm}^{-1}$ ) 2924, 2854, 1735, 1446, 1402, 1258, 1241, 1207, 1079

HRMS (ESI-TOF) m/z: [M+Na] calcd for $\mathrm{C}_{12} \mathrm{H}_{20} \mathrm{O}_{2} \mathrm{Na} 219.1261$, found 219.1355

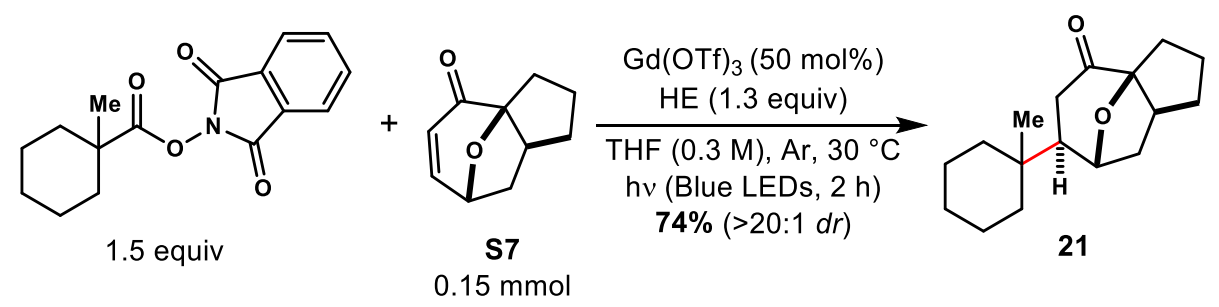


Ketone 21: According to the general procedure, enone $\mathbf{S} 7^{18}(25.4 \mathrm{mg}, 0.15 \mathrm{mmol})$ was used as the acceptor. Purification of the product by flash column chromatography $\left(\mathrm{SiO}_{2}, 1: 0 \rightarrow 9: 1\right.$ Hex/EtOAc) provided ketone 21 (30.2 mg, 74\%) as a colorless solid. $\mathrm{R}_{\mathrm{f}}=0.44$ (9:1 Hex/EtOAc, visualized with $\mathrm{KMnO}_{4}$ ). X-Ray quality crystals were obtained by slow evaporation from dichloromethane.

${ }^{1} \mathbf{H}$ NMR $\left(600 \mathrm{MHz}, \mathrm{CDCl}_{3}\right) \delta 4.51(\mathrm{dd}, J=2.6,5.9 \mathrm{~Hz}, 1 \mathrm{H}), 2.53($ app q, $J=7.2 \mathrm{~Hz}, 1 \mathrm{H}), 2.48$ $(\mathrm{dd}, J=11.8,15.8 \mathrm{~Hz}, 1 \mathrm{H}), 2.24(\mathrm{dd}, J=5.9,15.8 \mathrm{~Hz}, 1 \mathrm{H}), 2.14-2.07(\mathrm{~m}, 1 \mathrm{H}), 1.91(\mathrm{dd}, J=8.9$, $11.7 \mathrm{~Hz}, 1 \mathrm{H}), 1.87-1.71(\mathrm{~m}, 4 \mathrm{H}), 1.68(\mathrm{dt}, J=6.2,11.8 \mathrm{~Hz}, 1 \mathrm{H}), 1.55-1.35(\mathrm{~m}, 8 \mathrm{H}), 1.31-1.16$ $(\mathrm{m}, 4 \mathrm{H}), 0.93(\mathrm{~s}, 3 \mathrm{H})$

${ }^{13} \mathrm{C}$ NMR $\left(150 \mathrm{MHz}, \mathrm{CDCl}_{3}\right) \delta 214.6,97.2,76.6,47.8,46.6,42.2,35.7,35.5,35.4,33.1,31.5$, $26.3,25.6,21.7,21.6,19.3$

IR (thin film, $\mathrm{cm}^{-1}$ ) 2926, 2862, 1719, 1447, 1130, 1043, 988

Melting Point: $52-54^{\circ} \mathrm{C}$

HRMS (ESI-TOF) m/z: [M+H] $]^{+}$calcd for $\mathrm{C}_{17} \mathrm{H}_{27} \mathrm{O}_{2} 263.2011$, found 263.2019

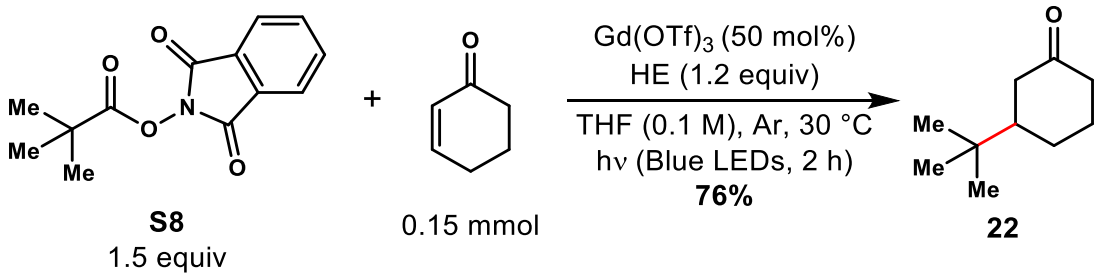

Ketone 22: According to the general procedure, NHPI ester $\mathbf{S 8}^{19}$ (56 $\mathrm{mg}, 0.22 \mathrm{mmol}$ ) was used as the radical precursor with cyclohexenone $(15 \mu \mathrm{L}, 0.15 \mathrm{mmol})$ as the acceptor. Purification of the product by flash column chromatography $\left(\mathrm{SiO}_{2}, 1: 0 \rightarrow 9: 1 \mathrm{Hex} /\right.$ EtOAc) provided ketone 22 (17.5 $\mathrm{mg}, 76 \%$ ) as a colorless oil. Characterization data acquired for the compound matched those previously reported. ${ }^{20}$

${ }^{1} \mathbf{H}$ NMR $\left(600 \mathrm{MHz}, \mathrm{CDCl}_{3}\right) \delta 2.46-2.41(\mathrm{~m}, 1 \mathrm{H}), 2.38-2.32(\mathrm{~m}, 1 \mathrm{H}), 2.23(\mathrm{dt}, J=6.5,13.9 \mathrm{~Hz}$, 1H), 2.13-2.07 (m, 1H), $2.04(\mathrm{t}, J=13.4 \mathrm{~Hz}, 1 \mathrm{H}), 1.97-1.90(\mathrm{~m}, 1 \mathrm{H}), 1.60-1.45(\mathrm{~m}, 2 \mathrm{H}), 1.32$ $(\mathrm{dq}, J=3.5,12.7 \mathrm{~Hz}, 1 \mathrm{H}), 0.89(\mathrm{~s}, 9 \mathrm{H})$

${ }^{13}$ C NMR (125 MHz, $\left.\mathrm{CDCl}_{3}\right) \delta$ 213.1, 49.4, 43.7, 41.4, 32.8, 27.2, 26.2, 25.7 

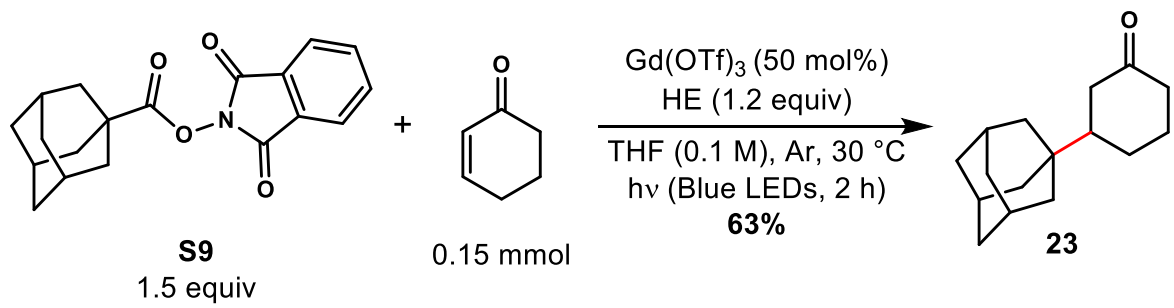

Ketone 23: According to the general procedure, NHPI ester $\mathbf{5 9}{ }^{21}$ (73 $\mathrm{mg}, 0.22 \mathrm{mmol}$ ) was used as the radical precursor with cyclohexenone $(15 \mu \mathrm{L}, 0.15 \mathrm{mmol})$ as the acceptor. Purification of the product by flash column chromatography $\left(\mathrm{SiO}_{2}, 1: 0 \rightarrow 9: 1 \mathrm{Hex} /\right.$ EtOAc) provided ketone 23 (22 $\mathrm{mg}, 63 \%$ ) as a colorless oil. Characterization data acquired for the compound matched those previously reported..$^{22}$

${ }^{1} \mathbf{H}$ NMR $\left(600 \mathrm{MHz}, \mathrm{CDCl}_{3}\right) \delta 2.47-2.40(\mathrm{~m}, 1 \mathrm{H}), 2.37-2.31(\mathrm{~m}, 1 \mathrm{H}), 2.22(\mathrm{dt}, J=6.4,13.9 \mathrm{~Hz}$, $1 \mathrm{H}), 2.12-2.02(\mathrm{~m}, 2 \mathrm{H}), 2.00-1.90(\mathrm{~m}, 4 \mathrm{H}), 1.75-1.67(\mathrm{~m}, 3 \mathrm{H}), 1.65-1.59(\mathrm{~m}, 3 \mathrm{H}), 1.56-1.46(\mathrm{~m}$, $7 \mathrm{H}), 1.37-1.26(\mathrm{~m}, 2 \mathrm{H})$

${ }^{13}$ C NMR $\left(150 \mathrm{MHz}, \mathrm{CDCl}_{3}\right) \delta 213.6,49.7,42.2,41.6,39.5,37.3,34.5,28.7,25.8,24.7$

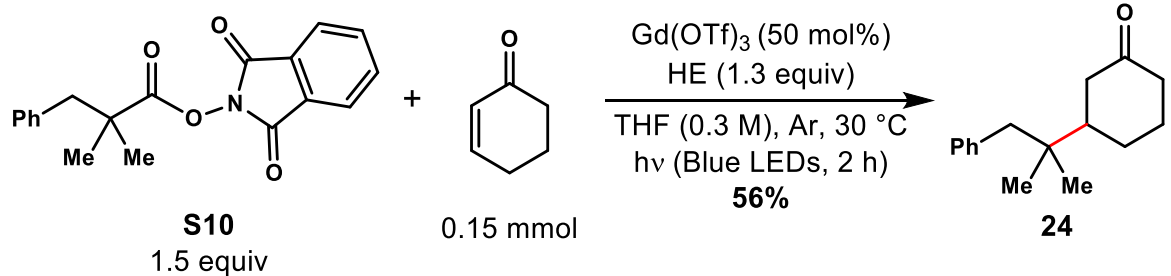

Ketone 24: According to the general procedure, NHPI ester $\mathbf{S 1 0}^{23}$ (85 $\mathrm{mg}, 0.22 \mathrm{mmol}$ ) was used as the radical precursor with cyclohexenone $(15 \mu \mathrm{L}, 0.15 \mathrm{mmol})$ as the acceptor. Purification of the product by flash column chromatography $\left(\mathrm{SiO}_{2}, 1: 0 \rightarrow 9: 1 \mathrm{Hex} /\right.$ EtOAc) provided ketone 24 $(19.5 \mathrm{mg}, 56 \%)$ as a colorless oil. $\mathrm{R}_{\mathrm{f}}=0.30$ (9:1 Hex/EtOAc, visualized with $\left.\mathrm{KMnO}_{4}\right)$.

${ }^{1} \mathbf{H}$ NMR $\left(600 \mathrm{MHz}, \mathrm{CDCl}_{3}\right) \delta 7.28-7.23(\mathrm{~m}, 2 \mathrm{H}), 7.20(\operatorname{app} \mathrm{t}, J=7.3 \mathrm{~Hz}, 1 \mathrm{H}), 7.10(\mathrm{~d}, J=7.3$ $\mathrm{Hz}, 2 \mathrm{H}), 2.57(\mathrm{~d}, J=13.1 \mathrm{~Hz}, 1 \mathrm{H}), 2.54(\mathrm{~d}, J=13.1 \mathrm{~Hz}, 1 \mathrm{H}), 2.54-2.49(\mathrm{~m}, 1 \mathrm{H}), 2.41-2.35(\mathrm{~m}$, $1 \mathrm{H}), 2.26(\mathrm{dt}, J=6.5,13.8 \mathrm{~Hz}, 1 \mathrm{H}), 2.18-2.09(\mathrm{~m}, 2 \mathrm{H}), 2.07-2.02(\mathrm{~m}, 1 \mathrm{H}), 1.62-1.51(\mathrm{~m}, 2 \mathrm{H})$, $1.42(\mathrm{dq}, J=3.3,12.6 \mathrm{~Hz}, 1 \mathrm{H}), 0.85(\mathrm{~s}, 3 \mathrm{H}), 0.84(\mathrm{~s}, 3 \mathrm{H})$

${ }^{13} \mathrm{C}$ NMR $\left(150 \mathrm{MHz}, \mathrm{CDCl}_{3}\right) \delta 212.7,138.7,130.7,127.8,126.0,47.5,45.7,43.6,41.4,36.6$, $26.1,25.5,24.3,24.1$

IR (thin film, cm $\mathrm{cm}^{-1}$ ) 2960, 2863, 1707, 1494, 1450, 1387, 1366, 1318, 1269, 1231

HRMS (ESI-TOF) m/z: $[\mathrm{M}+\mathrm{H}]^{+}$calcd for $\mathrm{C}_{16} \mathrm{H}_{23} \mathrm{O} 231.1749$, found 231.1753 


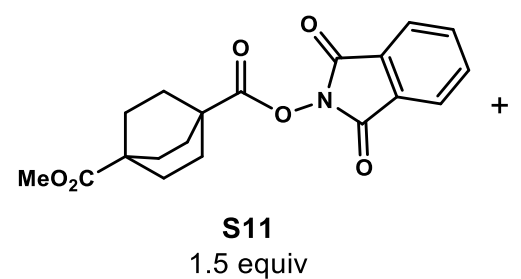

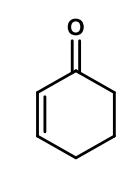

$0.15 \mathrm{mmol}$

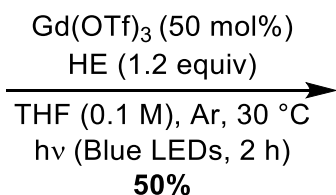

$50 \%$

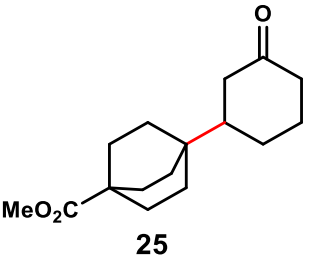

25

Ketone 25: According to the general procedure, NHPI ester $\mathbf{S 1 1}^{24}$ (80 $\left.\mathrm{mg}, 0.22 \mathrm{mmol}\right)$ was used as the radical precursor with cyclohexenone $(15 \mu \mathrm{L}, 0.15 \mathrm{mmol})$ as the acceptor. Purification of the product by flash column chromatography $\left(\mathrm{SiO}_{2}, 1: 0 \rightarrow 4: 1 \mathrm{Hex} /\right.$ Acetone) provided ketone 25 $(20 \mathrm{mg}, 50 \%)$ as a colorless solid. $\mathrm{R}_{\mathrm{f}}=0.24\left(4: 1 \mathrm{Hex} /\right.$ Acetone, visualized with $\left.\mathrm{KMnO}_{4}\right)$.

${ }^{1} \mathbf{H}$ NMR $\left(600 \mathrm{MHz}, \mathrm{CDCl}_{3}\right) \delta 3.63(\mathrm{~s}, 3 \mathrm{H}), 3.28-2.31(\mathrm{~m}, 2 \mathrm{H}), 2.21$ (dt, $\left.J=6.4,13.9 \mathrm{~Hz}, 1 \mathrm{H}\right)$, 2.11-2.05 (m, 1H), $1.98(\mathrm{t}, J=13.4 \mathrm{~Hz}, 1 \mathrm{H}), 1.87$ (app. d, $J=13.1 \mathrm{~Hz}, 1 \mathrm{H}), 1.76$ (dd, $J=6.4,9.4$ $\mathrm{Hz}, 6 \mathrm{H}), 1.57-1.47(\mathrm{~m}, 1 \mathrm{H}), 1.46-1.36(\mathrm{~m}, 7 \mathrm{H}), 1.30-1.21(\mathrm{~m}, 1 \mathrm{H})$

${ }^{13} \mathrm{C}$ NMR $\left(150 \mathrm{MHz}, \mathrm{CDCl}_{3}\right) \delta 212.6,178.3,51.6,47.2,43.2,41.4,38.6,33.2,28.3,27.6,25.8$, 25.6

IR (thin film, $\mathrm{cm}^{-1}$ ) 2947, 2861, 1716, 1431, 1232, 1125, 1069

HRMS (ESI-TOF) m/z: [M+Na $]^{+}$calcd for $\mathrm{C}_{16} \mathrm{H}_{24} \mathrm{O}_{3} \mathrm{Na} 287.1623$, found 287.1621

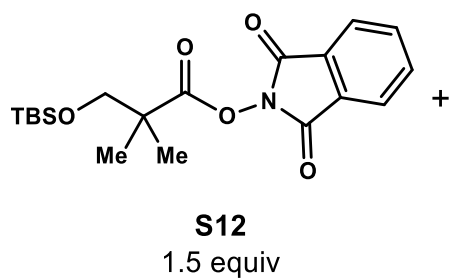

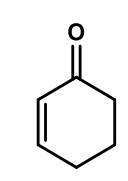

$0.15 \mathrm{mmol}$

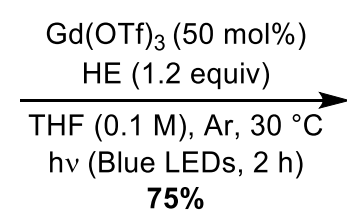

$75 \%$

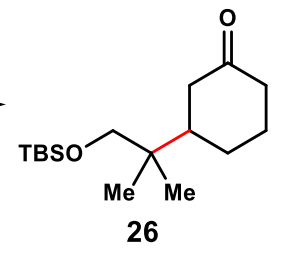

Ketone 26: According to the general procedure, NHPI ester $\mathbf{S 1 2}^{24}$ (85 $\mathrm{mg}, 0.22 \mathrm{mmol}$ ) was used as the radical precursor with cyclohexenone $(15 \mu \mathrm{L}, 0.15 \mathrm{mmol})$ as the acceptor. Purification of the product by flash column chromatography $\left(\mathrm{SiO}_{2}, 1: 0 \rightarrow 9: 1 \mathrm{Hex} / \mathrm{EtOAc}\right.$ ) provided ketone 26 (32 $\mathrm{mg}, 75 \%)$ as a colorless oil. $\mathrm{R}_{\mathrm{f}}=0.37\left(5: 1 \mathrm{Hex} / \mathrm{EtOAc}\right.$, visualized with $\left.\mathrm{KMnO}_{4}\right)$.

${ }^{1} \mathbf{H}$ NMR $\left(600 \mathrm{MHz}, \mathrm{CDCl}_{3}\right) \delta 3.32(\mathrm{~d}, J=9.7 \mathrm{~Hz}, 1 \mathrm{H}), 3.28(\mathrm{~d}, J=9.7 \mathrm{~Hz}, 1 \mathrm{H}), 2.42-2.33(\mathrm{~m}$, $1 \mathrm{H}), 2.14-2.05(\mathrm{~m}, 2 \mathrm{H}), 1.92-1.86(\mathrm{~m}, 1 \mathrm{H}), 1.81-1.73(\mathrm{~m}, 1 \mathrm{H}), 1.55(\mathrm{qt}, J=3.9,13.3 \mathrm{~Hz}, 1 \mathrm{H})$, $1.35(\mathrm{dq}, J=3.5,12.7 \mathrm{~Hz}, 1 \mathrm{H}), 0.88(\mathrm{~s}, 9 \mathrm{H}), 0.83(\mathrm{~s}, 3 \mathrm{H}), 0.82(\mathrm{~s}, 3 \mathrm{H}), 0.02(\mathrm{~s}, 3 \mathrm{H}), 0.01(\mathrm{~s}, 3 \mathrm{H})$

${ }^{13} \mathrm{C}$ NMR $\left(150 \mathrm{MHz}, \mathrm{CDCl}_{3}\right) \delta 213.0,70.2,44.5,43.4,41.5,37.5,25.9,25.88,25.7,22.1,21.6$, $18.2,-5.5,-5.6$

IR (thin film, $\mathrm{cm}^{-1}$ ) 2954, 2928, 2885, 2856, 1712, 1471, 1250, 1099

HRMS (ESI-TOF) m/z: [M+Na] $]^{+}$calcd for $\mathrm{C}_{16} \mathrm{H}_{32} \mathrm{O}_{2} \mathrm{SiNa} 307.2069$, found 307.2044 

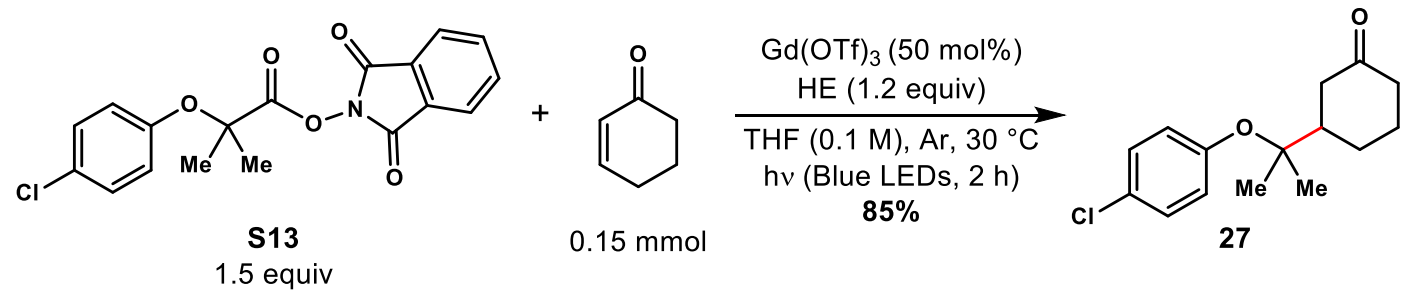

Ketone 27: According to the general procedure, NHPI ester $\mathbf{S 1 3}^{24}(81 \mathrm{mg}, 0.22 \mathrm{mmol})$ was used as the radical precursor with cyclohexenone $(15 \mu \mathrm{L}, 0.15 \mathrm{mmol})$ as the acceptor. Purification by flash column chromatography $\left(\mathrm{SiO}_{2}, 1: 0 \rightarrow 9: 1 \mathrm{Hex} / \mathrm{EtOAc}\right)$ provided ketone $27(34 \mathrm{mg}, 85 \%)$ as a colorless oil. $\mathrm{R}_{\mathrm{f}}=0.44\left(4: 1 \mathrm{Hex} /\right.$ EtOAc, visualized with $\left.\mathrm{KMnO}_{4}\right)$

${ }^{1} \mathbf{H}$ NMR $\left(600 \mathrm{MHz}, \mathrm{CDCl}_{3}\right) \delta 7.22(\mathrm{~d}, J=8.5 \mathrm{~Hz}, 2 \mathrm{H}), 6.87(\mathrm{~d}, J=8.5 \mathrm{~Hz}, 2 \mathrm{H}), 2.66-2.60(\mathrm{~m}$, $1 \mathrm{H}), 2.44-2.38$ (m, 1H), 2.34 (app t, $J=13.4 \mathrm{~Hz}, 1 \mathrm{H}), 2.32-2.26(\mathrm{~m}, 1 \mathrm{H}), 2.20-2.10(\mathrm{~m}, 2 \mathrm{H})$, $2.20-1.97(\mathrm{~m}, 1 \mathrm{H}), 1.68-1.57(\mathrm{~m}, 1 \mathrm{H}), 1.23(\mathrm{~s}, 3 \mathrm{H}), 1.21(\mathrm{~s}, 3 \mathrm{H})$

${ }^{13} \mathrm{C}$ NMR $\left(150 \mathrm{MHz}, \mathrm{CDCl}_{3}\right) \delta 212.0,153.6,129.0,128.8,125.4,81.8,49.0,43.2,41.3,25.9$, $25.1,24.3,24.1$

IR (thin film, $\mathrm{cm}^{-1}$ ) 2877, 2935, 2868, 1708, 1486, 1385, 1369, 1228, 1130, 1089, 846

HRMS (ESI-TOF) m/z: [M+Na] $]^{+}$calcd for $\mathrm{C}_{15} \mathrm{H}_{19} \mathrm{O}_{2} \mathrm{ClNa} 289.0971$, found 289.0968
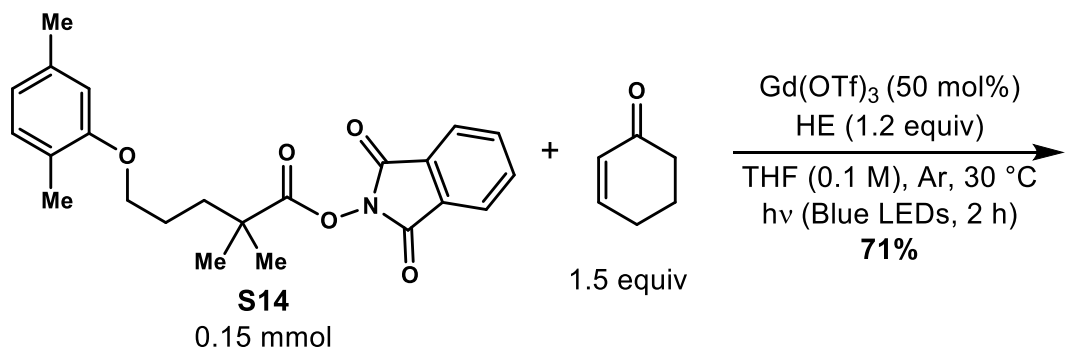

Ketone 28: According to the general procedure with the exception of using excess acceptor, NHPI ester $\mathbf{S 1 4}{ }^{21}$ (59 $\left.\mathrm{mg}, 0.15 \mathrm{mmol}\right)$ was used as the radical precursor with cyclohexenone $(22 \mu \mathrm{L}$, $0.225 \mathrm{mmol}, 1.5$ equiv) as the acceptor. Purification of the product by flash column chromatography ( $\mathrm{SiO}_{2}, 1: 0 \rightarrow 9: 1 \mathrm{Hex} /$ EtOAc) provided ketone $\mathbf{2 8}(32 \mathrm{mg}, 71 \%)$ as a colorless oil. $\mathrm{R}_{\mathrm{f}}=0.39(5: 1 \mathrm{Hex} / \mathrm{EtOAc})$.

${ }^{1}$ H NMR $\left(600 \mathrm{MHz}, \mathrm{CDCl}_{3}\right) \delta 7.00(\mathrm{~d}, J=7.4 \mathrm{~Hz}, 1 \mathrm{H}), 6.65(\mathrm{~d}, J=7.4 \mathrm{~Hz}, 1 \mathrm{H}), 6.61(\mathrm{~s}, 1 \mathrm{H})$, 3.95-3.87 (m, 2H), 2.45-2.40 (m, 1H), 2.39-2.34 (m, 1H), $2.30(\mathrm{~s}, 3 \mathrm{H}), 2.24(\mathrm{dt}, J=6.5,13.9 \mathrm{~Hz}$, $1 \mathrm{H}), 2.17(\mathrm{~s}, 3 \mathrm{H}), 2.14-2.05(\mathrm{~m}, 2 \mathrm{H}), 1.96-1.89(\mathrm{~m}, 1 \mathrm{H}), 1.72(\mathrm{dq}, J=6.7,9.4 \mathrm{~Hz}, 2 \mathrm{H}), 1.63(\mathrm{tt}, J$ $=3.3,12.6 \mathrm{~Hz}, 1 \mathrm{H}), 1.58-1.52(\mathrm{~m}, 1 \mathrm{H}), 1.49-1.32(\mathrm{~m}, 3 \mathrm{H}), 0.94(\mathrm{~s}, 3 \mathrm{H}), 0.89(\mathrm{~s}, 3 \mathrm{H})$

${ }^{13} \mathrm{C}$ NMR $\left(150 \mathrm{MHz}, \mathrm{CDCl}_{3}\right) \delta 212.9,157.0,136.5,130.3,123.5,120.6,111.9,68.3,47.1,43.3$, $41.4,36.5,34.9,25.8,25.7,24.5,24.4,23.8,21.4,15.8$

IR (thin film, $\mathrm{cm}^{-1}$ ) 2952, 2863, 1708, 1508, 1470, 1414, 1388, 1284, 1263, 1157, 1129, 1035, 802 
HRMS (ESI-TOF) m/z: $[\mathrm{M}+\mathrm{Na}]^{+}$calcd for $\mathrm{C}_{20} \mathrm{H}_{30} \mathrm{O}_{2} \mathrm{Na} 325.2144$, found 325.2143
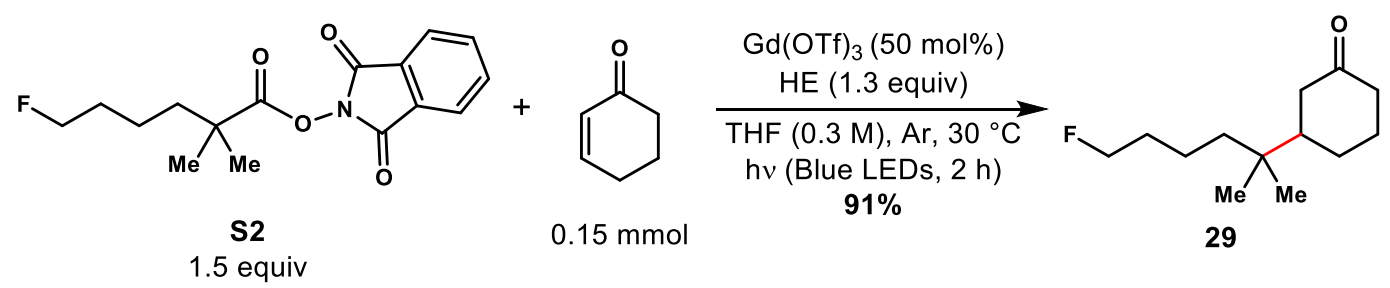

Ketone 29: According to the general procedure, NHP ester S2 $(69.7 \mathrm{mg}, 0.225 \mathrm{mmol})$ was used as the radical precursor with cyclohexenone $(15 \mu \mathrm{L}, 0.15 \mathrm{mmol})$ as the acceptor. Purification of the product by flash column chromatography $\left(\mathrm{SiO}_{2}, 1: 0 \rightarrow 9: 1 \mathrm{Hex} / \mathrm{EtOAc}\right)$ provided ketone 29 $(29.2 \mathrm{mg}, 91 \%)$ as a colorless foam. $\mathrm{R}_{\mathrm{f}}=0.30\left(9: 1 \mathrm{Hex} / \mathrm{EtOAc}\right.$, visualized with $\left.\mathrm{KMnO}_{4}\right)$.

${ }^{1}$ H NMR $\left(600 \mathrm{MHz}, \mathrm{CDCl}_{3}\right) \delta 4.51-4.46(\mathrm{~m}, 1 \mathrm{H}), 4.43-4.37(\mathrm{~m}, 1 \mathrm{H}), 2.41-2.31(\mathrm{~m}, 2 \mathrm{H}), 2.27-$ $2.19(\mathrm{~m}, 1 \mathrm{H}), 2.13-2.03(\mathrm{~m}, 2 \mathrm{H}), 1.91-1.84(\mathrm{~m}, 1 \mathrm{H}), 1.70-1.50(\mathrm{~m}, 5 \mathrm{H}), 1.37-1.29(\mathrm{~m}, 3 \mathrm{H}), 1.28-$ $1.20(\mathrm{~m}, 2 \mathrm{H}), 0.87(\mathrm{~s}, 3 \mathrm{H}), 0.85(\mathrm{~s}, 3 \mathrm{H})$

${ }^{13} \mathbf{C}$ NMR $\left(150 \mathrm{MHz}, \mathrm{CDCl}_{3}\right) \delta 213.0,84.6\left(\mathrm{C}-\mathrm{F},{ }^{1} \mathrm{JC}-\mathrm{F}=164 \mathrm{~Hz}\right), 83.6\left(\mathrm{C}-\mathrm{F},{ }^{1} \mathrm{JC}-\mathrm{F}=164 \mathrm{~Hz}\right)$, 47.2, 45.4, 43.3, 41.4, 40.0, 35.1, 31.4 (C-F, $\left.\left.{ }^{3} \mathrm{JC}-\mathrm{F}=19.5 \mathrm{~Hz}\right), 31.2 \mathrm{C}-\mathrm{F},{ }^{3} \mathrm{JC}-\mathrm{F}=19.5 \mathrm{~Hz}\right), 28.4$, $25.8\left(\mathrm{C}-\mathrm{F},{ }^{4} \mathrm{JC}-\mathrm{F}=13.5 \mathrm{~Hz}\right), 25.7\left(\mathrm{C}-\mathrm{F},{ }^{4} \mathrm{JC}-\mathrm{F}=13.5 \mathrm{~Hz}\right), 24.5\left(\mathrm{C}-\mathrm{F},{ }^{2} \mathrm{JC}-\mathrm{F}=20.0 \mathrm{~Hz}\right), 24.3(\mathrm{C}-$ $\left.\mathrm{F},{ }^{2} \mathrm{JC}-\mathrm{F}=20.0 \mathrm{~Hz}\right), 19.48\left(\mathrm{C}-\mathrm{F},{ }^{5} \mathrm{JC}-\mathrm{F}=5.2 \mathrm{~Hz}\right), 19.44\left(\mathrm{C}-\mathrm{F},{ }^{5} \mathrm{JC}-\mathrm{F}=5.2 \mathrm{~Hz}\right)$

IR (thin film, $\mathrm{cm}^{-1}$ ) 2957, 2868, 1710, 1472, 1388, 1367, 1032

HRMS (ESI-TOF) m/z: [M+Na] $]^{+}$calcd for $\mathrm{C}_{13} \mathrm{H}_{23} \mathrm{FONa} 237.1631$, found 237.1636
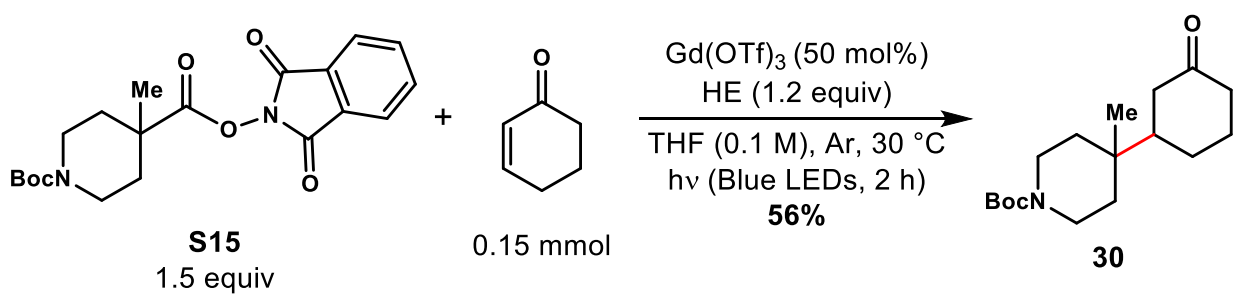

Ketone 30: According to the general procedure, NHP ester $\mathbf{S 1 5}^{3}(87 \mathrm{mg}, 0.22 \mathrm{mmol}$ ) was used as the radical precursor with cyclohexenone $(15 \mu \mathrm{L}, 0.15 \mathrm{mmol})$ as the acceptor. Purification of the product by flash column chromatography $\left(\mathrm{SiO}_{2}, 1: 0 \rightarrow 85: 15 \mathrm{Hex} / \mathrm{EtOAc}\right)$ provided ketone 30 (25 $\mathrm{mg}, 56 \%)$ as a colorless oil. $\mathrm{R}_{\mathrm{f}}=0.34\left(4: 1 \mathrm{Hex} / \mathrm{EtOAc}\right.$, visualized with $\left.\mathrm{KMnO}_{4}\right)$.

${ }^{1} \mathbf{H}$ NMR $\left(500 \mathrm{MHz}, \mathrm{CDCl}_{3}\right) \delta$ 3.87-3.65 (m, 2H), 3.05-2.93 (m, 2H), 2.43-2.33 (m, 2H), $2.24(\mathrm{dt}$, $J=6.5,14.1 \mathrm{~Hz}, 1 \mathrm{H}), 2.15-2.02(\mathrm{~m}, 2 \mathrm{H}), 1.94-1.83(\mathrm{~m}, 1 \mathrm{H}), 1.61-1.49(\mathrm{~m}, 3 \mathrm{H}), 1.44(\mathrm{~s}, 9 \mathrm{H}), 1.42-$ $1.36(\mathrm{~m}, 2 \mathrm{H}), 1.36-1.27(\mathrm{~m}, 2 \mathrm{H}), 0.91(\mathrm{~s}, 3 \mathrm{H})$

${ }^{13} \mathrm{C}$ NMR $\left(125 \mathrm{MHz}, \mathrm{CDCl}_{3}\right) \delta 212.3,154.9,79.4,48.8,42.5,41.5,39.7,35.3,33.8,28.5,25.5$, 25.0, 17.6 
IR (thin film, cm-1) 2934, 2864, 1690, 1477, 1424, 1389, 1248, 1159

HRMS (ESI-TOF) m/z: [M+Na $]^{+}$calcd for $\mathrm{C}_{17} \mathrm{H}_{29} \mathrm{NO}_{3} \mathrm{Na} 318.2045$, found 318.2044
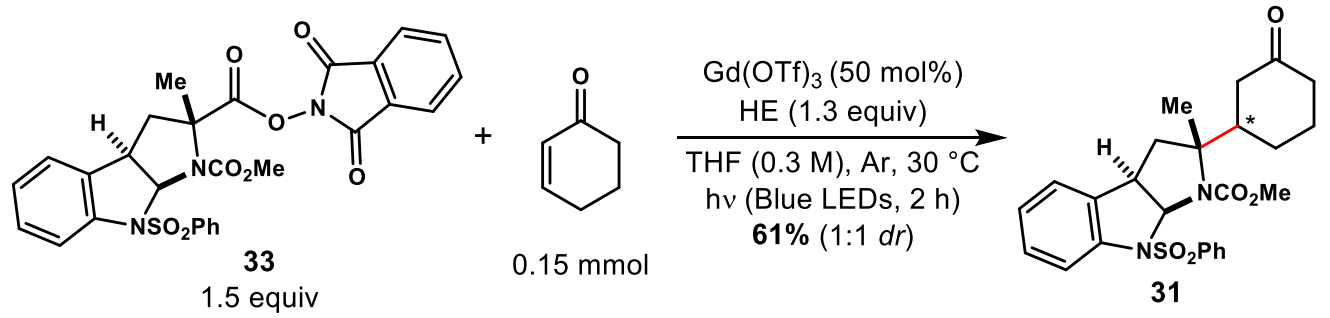

Ketone 31: According to the general procedure, NHPI ester 33 (126 mg, $0.225 \mathrm{mmol})$ was used as the radical precursor with cyclohexenone $(15 \mu \mathrm{L}, 0.15 \mathrm{mmol})$ as the acceptor. Purification of the product by flash column chromatography $\left(\mathrm{SiO}_{2}, 1: 0 \rightarrow 9: 1 \mathrm{Hex} / \mathrm{EtOAc}\right)$ provided ketone 31 (43 $\mathrm{mg}, 61 \%)$ as a colorless foam and an inseparable $1: 1$ mixture of diastereomers. $\mathrm{R}_{\mathrm{f}}=0.32(1: 1$ $\mathrm{Hex} / \mathrm{EtOAc})$.

${ }^{1} \mathbf{H}$ NMR $\left(600 \mathrm{MHz}, \mathrm{CDCl}_{3}\right) \delta$ 7.94-7.82 (m, 2H), 7.67-7.39 (m, 4H), 7.25-7.18 (m, 1H), 7.09$6.97(\mathrm{~m}, 2 \mathrm{H}), 6.21(\mathrm{~d}, J=7.2 \mathrm{~Hz}, 0.5 \mathrm{H}), 5.41(\mathrm{dd}, J=2.7,8.0 \mathrm{~Hz}, 0.5 \mathrm{H}), 3.78(\mathrm{~s}, 1.5 \mathrm{H}), 3.67(\mathrm{~s}$, $1.5 \mathrm{H}), 3.57-3.50(\mathrm{~m}, 0.5 \mathrm{H}), 3.45(\mathrm{q}, J=7.4 \mathrm{~Hz}, 0.5 \mathrm{H}), 2.51-2.37(\mathrm{~m}, 0.5 \mathrm{H}), 2.32-2.19(\mathrm{~m}, 1 \mathrm{H})$, 2.17-2.05 (m, 2H), 2.03-1.91 (m, 2.5H), $1.97(\mathrm{~s}, 1.5 \mathrm{H}), 1.90-1.81(\mathrm{~s}, 1 \mathrm{H}), 1.75-1.56(\mathrm{~m}, 1 \mathrm{H})$, $1.42(\mathrm{~s}, 1.5 \mathrm{H}), 1.41-1.20(\mathrm{~m}, 3 \mathrm{H})$

${ }^{13} \mathrm{C}$ NMR $\left(125 \mathrm{MHz}, \mathrm{CDCl}_{3}\right) \delta 211.2,205.3,155.6,140.7,140.2,137.8,133.5,133.2,132.2$, $129.2,128.9,128.8,128.7,128.4,127.6,127.4,125.2,124.7,124.65,123.7,122.3,122.2,116.7$, $115.5,111.4,83.0,77.3,75.0,69.0,52.6,52.5,48.2,46.2,44.5,43.4,42.4,41.2,30.6,30.1,29.7$, $27.2,25.4,23.7,20.4$

IR (thin film, $\mathrm{cm}^{-1}$ ) 2951, 1704, 1531, 1476, 1461, 1445, 1343, 1231, 1171, 1090

HRMS (ESI-TOF) m/z: [M+Na $]^{+}$calcd for $\mathrm{C}_{25} \mathrm{H}_{28} \mathrm{~N}_{2} \mathrm{O}_{5} \mathrm{SNa} 491.1617$, found 491.1615
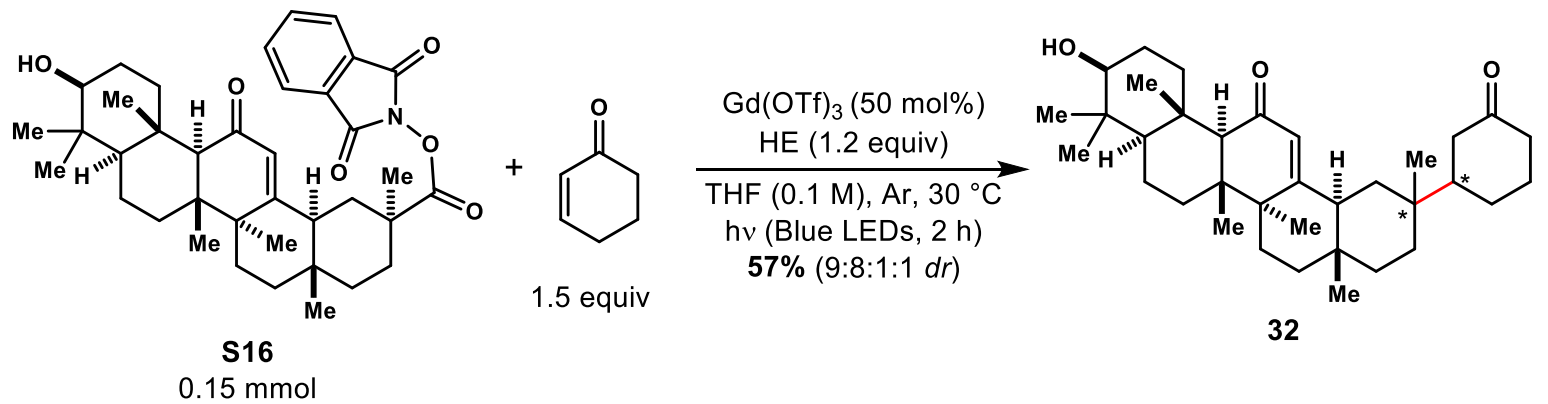

Ketone 32: According to the general procedure with the exception of using excess acceptor, NHPI ester $\mathbf{S 1 6}^{21}$ (92 $\left.\mathrm{mg}, 0.15 \mathrm{mmol}\right)$ was used as the radical precursor with cyclohexenone $(22 \mu \mathrm{L}$, $0.225 \mathrm{mmol})$ as the acceptor. Purification of the product by flash column chromatography $\left(\mathrm{SiO}_{2}\right.$, 
$1: 0 \rightarrow 3: 2 \mathrm{Hex} / \mathrm{EtOAc})$ provided ketone $\mathbf{3 2}(45 \mathrm{mg}, 57 \%)$ as a colorless oil and as an inseparable 9:8:1:1 mixture of diastereomers. $\mathrm{R}_{\mathrm{f}}=0.59(2: 3 \mathrm{Hex} / \mathrm{EtOAc})$.

${ }^{1}$ H NMR $\left(600 \mathrm{MHz}, \mathrm{CDCl}_{3}\right) \delta 5.63(\mathrm{~s}, 0.06 \mathrm{H}), 5.59(\mathrm{~s}, 0.46 \mathrm{H}), 5.57(\mathrm{~s}, 0.40 \mathrm{H}), 5.55(\mathrm{~s}, 0.07 \mathrm{H})$, 3.27-3.18 (m, 1H), 2.82-2.74 (m, 1H), 2.47-2.05 (m, 8H), 2.20-1.87 (m, 2H), 1.84-1.77 (m, 1H), $1.67-1.38(\mathrm{~m}, 12 \mathrm{H}), 1.38-1.29(\mathrm{~m}, 6 \mathrm{H}), 1.23-1.19(\mathrm{~m}, 1 \mathrm{H}), 1.18-1.09(\mathrm{~m}, 7 \mathrm{H}), 1.00(\mathrm{~s}, 3 \mathrm{H}), 0.99-$ $0.92(\mathrm{~m}, 2 \mathrm{H}), 0.87-0.84(\mathrm{~m}, 2 \mathrm{H}), 0.86(\mathrm{~s}, 3 \mathrm{H}), 0.80(\mathrm{~s}, 3 \mathrm{H}), 0.72-0.65(\mathrm{~m}, 1 \mathrm{H})$

${ }^{13}$ C NMR (150 MHz, $\left.\mathrm{CDCl}_{3}\right) \delta$ (Only the Two Major Products' Peaks are Listed) 212.75, 212.71, 200.3, 200.2, 170.2, 170.0, 128.44, 128.42, 78.80, 78.77, 61.9, 61.8, 55.0, 54.9, 51.32, 51.26, 47.2, 47.0, 46.5, 46.1, 45.5, 43.40, 43.36, 42.8, 42.7, 42.5, 42.2, 42.1, 41.9, 41.6, 41.4, 41.3, 39.2, 39.18, $39.15,37.2,37.1,36.04,36.00,35.9,35.7,35.5,35.2,32.8,32.5,32.4,32.1,30.8,30.4,28.72$, $28.69,28.1,27.4,26.4,26.3,25.6,25.6,25.3,25.0,24.2,24.1,23.6,23.2,18.77,18.75,17.5,17.3$, $17.0,16.4,15.6$

IR (thin film, cm ${ }^{-1}$ ) 3462, 2932, 2864, 1709, 1658, 1456, 1386, 1258, 1207, 1046, 994

HRMS (ESI-TOF) m/z: [M+Na] calcd for $\mathrm{C}_{35} \mathrm{H}_{54} \mathrm{O}_{3} \mathrm{Na} 545.3971$, found 545.3978 


\section{J. Procedure for the Coupling of Moderately Complex Fragments}

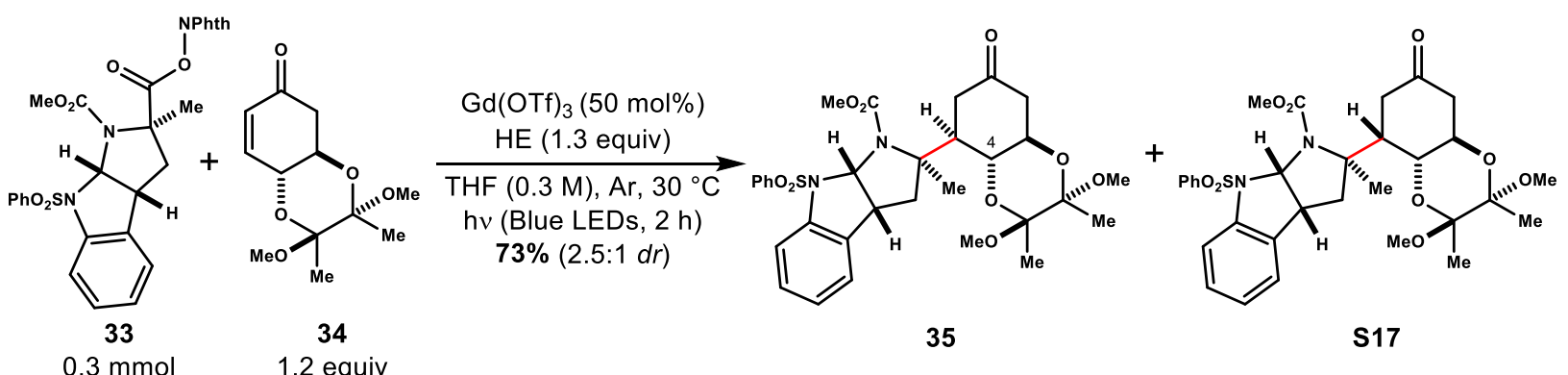

Ketones 35 and S17: NHPI ester 33 (164 mg, $0.29 \mathrm{mmol})$, enone 34 (87.8 mg, $0.36 \mathrm{mmol}, 1.2$ equiv), HE (102 mg, $0.40 \mathrm{mmol}, 1.3$ equiv), and $\mathrm{Gd}(\mathrm{OTf})_{3}(101 \mathrm{mg}, 0.16 \mathrm{mmol}, 50 \mathrm{~mol} \%$ ) were divided equally into three oven-dried 1-dram vials equipped with stir bars. The solids were then dissolved in THF ( $0.5 \mathrm{~mL}$ in each vial). The vials were sparged with Argon for 5 min and then sonicated for $30 \mathrm{sec}$. The vials were then placed on a stir plate equipped with two $34 \mathrm{~W}$ blue LEDs and a cardboard box (to block light pollution from entering the lab) with double-sided tape inside to hold the vials. The vials were placed in a triangle pattern approximately $4 \mathrm{~cm}$ from the lamps and stirred vigorously. The vials were irradiated by the lamps for $3 \mathrm{~h}$, while blowing a stream of air on the vials to maintain the reaction temperature around $30^{\circ} \mathrm{C}$. Following irradiation, a solution of 1:1 MeOH: $\mathrm{H}_{2} \mathrm{O}(1.5 \mathrm{~mL})$ and $\mathrm{DBU}(0.22 \mathrm{~mL}, 1.5 \mathrm{mmol}, 5$ equiv) was prepared and $0.5 \mathrm{~mL}$ of the solution was added to each vial. The reactions were stirred vigorously at $\mathrm{rt}$ for an additional 30 min. The reactions were then diluted with EtOAc $(5 \mathrm{~mL}$ for each vial) and poured into a separatory funnel. The mixture was then washed with $1 \mathrm{M} \mathrm{HCl}(30 \mathrm{~mL})$ and the organic layer was separated. The aqueous layer was washed with EtOAc $(2 \times 15 \mathrm{~mL})$. The combined organic layers were then dried over $\mathrm{MgSO}_{4}$, filtered, and concentrated to an orange foam. The crude residue was purified by flash column chromatography $\left(\mathrm{SiO}_{2}, 1: 0 \rightarrow 1: 1 \rightarrow 0: 1 \mathrm{Hex} / \mathrm{EtOAc}\right.$ ) provided ketone $\mathbf{3 5}^{25}$ (94 mg, $52 \%)$ as a colorless foam and ketone $\mathbf{S 1 7}(36.9 \mathrm{mg}, 21 \%)$ as a colorless oil. $\mathbf{R}_{\mathrm{f}}=0.45$ and $0.26(1: 1$ Hex/EtOAc), respectively.

\section{Ketone 35:}

${ }^{1} \mathbf{H}$ NMR $\left(600 \mathrm{MHz}, \mathrm{CDCl}_{3}\right) \delta$ 7.65-7.51 (m, 3H), $7.49(\mathrm{t}, J=7.4 \mathrm{~Hz}, 1 \mathrm{H}), 7.34(\mathrm{t}, J=7.6 \mathrm{~Hz}$, 2H), 7.27-7.21 (m, 1H), 7.09 (t, $J=7.3 \mathrm{~Hz}, 1 \mathrm{H}), 6.98(\mathrm{~d}, J=7.4 \mathrm{~Hz}, 1 \mathrm{H}), 6.08(\mathrm{~d}, J=4.3 \mathrm{~Hz}, 1 \mathrm{H})$, $4.30(\mathrm{dt}, J=5.7,11.5 \mathrm{~Hz}, 1 \mathrm{H}), 4.09(\mathrm{dd}, J=9.3,10.7 \mathrm{~Hz}, 1 \mathrm{H}), 3.80-3.65(\mathrm{~m}, 1 \mathrm{H}), 3.71(\mathrm{~s}, 3 \mathrm{H})$, 3.34-3.19 (m, 1H), 3.27 (s, 3H), 3.26 (s, 3H), 2.69-2.58 (m, 2H), 2.45-2.29 (m, 3H), 1.92-1.83 $(\mathrm{m}, 1 \mathrm{H}), 1.41(\mathrm{~s}, 3 \mathrm{H}), 1.31(\mathrm{~s}, 3 \mathrm{H}), 1.01(\mathrm{~s}, 3 \mathrm{H})$

${ }^{13} \mathrm{C}$ NMR $\left(125 \mathrm{MHz}, \mathrm{CDCl}_{3}\right) \delta 207.7,154.6,141.0,138.6,136.8,132.9,128.7,128.3,127.1$, 126.0, 123.7, 119.8, 99.6, 99.2, 83.1, 71.0, 65.8, 65.2, 51.6, 48.2, 48.1, 43.4, 42.7, 42.0, 39.2, 36.6, $28.2,17.7,17.6$

IR (thin film, $\mathrm{cm}^{-1}$ ) 2991, 2951, 2834, 1698, 1476, 1460, 1445, 1371, 1340, 1269, 1216, 1170, $1131,1116,1091,1051,1035$

HRMS (ESI-TOF) m/z: $[\mathrm{M}+\mathrm{Na}]^{+}$calcd for $\mathrm{C}_{31} \mathrm{H}_{38} \mathrm{~N}_{2} \mathrm{O}_{9} \mathrm{SNa}$ 637.2195, found 637.2196 
Optical Rotation $[\alpha]^{22.4}+123.7,[\alpha]^{22.5}{ }_{577}+129.1,[\alpha]^{22.6}{ }_{546}+145.5,[\alpha]^{22.7} 435+231.1(c=1.0$, $\mathrm{CHCl}_{3}$ )

\section{Ketone S17:}

${ }^{1} \mathbf{H}$ NMR $\left(500 \mathrm{MHz}, \mathrm{CDCl}_{3}\right) \delta 7.56(\mathrm{~d}, J=8.1 \mathrm{~Hz}, 1 \mathrm{H}), 7.58-7.49(\mathrm{~m}, 2 \mathrm{H}), 7.46(\mathrm{t}, J=7.2 \mathrm{~Hz}$, 1H), $7.31(\operatorname{app~t}, J=7.5 \mathrm{~Hz}, 2 \mathrm{H}), 7.28-7.23(\mathrm{~m}, 1 \mathrm{H}), 7.12(\mathrm{t}, J=7.4 \mathrm{~Hz}, 1 \mathrm{H}), 6.98(\mathrm{~d}, J=7.4 \mathrm{~Hz}$, $1 \mathrm{H}), 5.97$ (br s, 1H), 3.90-3.70 (m, 2H), $3.74(\mathrm{~s}, 3 \mathrm{H}), 3.32(\mathrm{~s}, 3 \mathrm{H}), 3.28-3.17(\mathrm{~m}, 2 \mathrm{H}), 3.20(\mathrm{~s}$, $3 \mathrm{H}), 2.58(\mathrm{dd}, J=4.3,15.5 \mathrm{~Hz}, 1 \mathrm{H}), 2.48-2.40(\mathrm{~m}, 1 \mathrm{H}), 2.36-2.20(\mathrm{~m}, 2 \mathrm{H}), 2.08-1.92(\mathrm{~m}, 2 \mathrm{H})$, $1.27(\mathrm{~s}, 3 \mathrm{H}), 1.25(\mathrm{~s}, 3 \mathrm{H}), 1.13(\mathrm{~s}, 3 \mathrm{H})$

${ }^{13} \mathrm{C}$ NMR $\left(125 \mathrm{MHz}, \mathrm{CDCl}_{3}\right) \delta 206.2,154.3,141.1,138.2,136.7,133.5,133.0,129.2,128.8$, $128.5,127.2,126.3,123.6,120.3,99.6,99.0,82.0,71.9,67.7,66.2,52.3,49.0,48.0,44.5,43.1$, $41.1,29.7,17.76,17.74$

IR (thin film, $\mathrm{cm}^{-1}$ ) 2992, 2949, 2833, 1704, 1477, 1460, 1445, 1375, 1363, 1343, 1270, 1218, $1169,1132,1090,1052,1037$

HRMS (ESI-TOF) m/z: $[\mathrm{M}+\mathrm{Na}]^{+}$calcd for $\mathrm{C}_{31} \mathrm{H}_{38} \mathrm{~N}_{2} \mathrm{O}_{9} \mathrm{SNa}$ 637.2195, found 637.2189

Optical Rotation $[\alpha]^{22.1}{ }_{D}+149.1,[\alpha]^{22.2}{ }_{577}+155.0,[\alpha]^{22.3}{ }_{546}+175.2,[\alpha]^{22.4} 435+292.2(c=1.0$, $\left.\mathrm{CHCl}_{3}\right)$ 


\section{K. Large Scale Fragment Coupling Procedure}

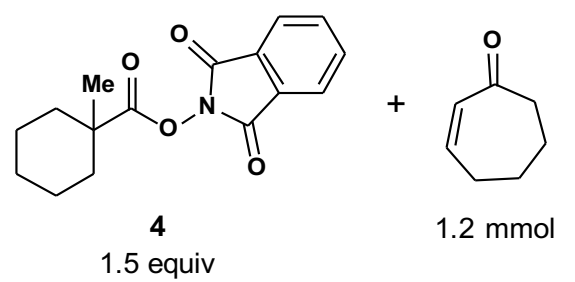

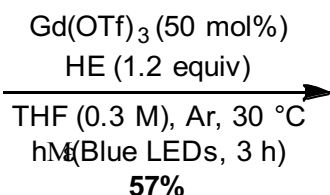

$57 \%$

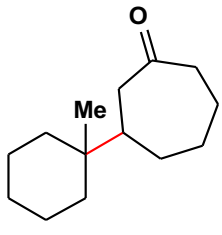

6

Ketone 6: NHPI ester 4 (520 mg, $1.8 \mathrm{mmol}, 1.5$ equiv), cyclohept-2-en-1-one (0.13 mL, $1.2 \mathrm{mmol}$, 1.0 equiv; purchased from Sigma-Aldrich), HE (370 mg, $1.46 \mathrm{mmol}, 1.2$ equiv), and Gd(OTf) 3 (362 mg, $0.6 \mathrm{mmol}, 50 \mathrm{~mol} \%$ ) were divided equally into four oven-dried 1-dram vials equipped with stir bars. The solids were then dissolved in THF (1 mL in each vial). The vials were sparged with Argon for $5 \mathrm{~min}$ and then sonicated for $30 \mathrm{sec}$. The vials were then placed on a stir plate equipped with two $34 \mathrm{~W}$ blue LEDs and a cardboard box (to block light pollution from entering the lab) with double-sided tape inside to hold the vials. The vials were placed in a square pattern approximately $4 \mathrm{~cm}$ from the lamps and stirred vigorously. The vials were irradiated by the lamps for $3 \mathrm{~h}$, while blowing a stream of air on the vials to maintain the reaction temperature around 30 ${ }^{\circ} \mathrm{C}$. Following irradiation, a solution of $1: 1 \mathrm{MeOH}: \mathrm{H}_{2} \mathrm{O}(3 \mathrm{~mL})$ and DBU $(0.9 \mathrm{~mL}, 6 \mathrm{mmol}, 5$ equiv) was prepared and $1 \mathrm{~mL}$ of the solution was added to each vial. The reactions were stirred vigorously at $\mathrm{rt}$ for an additional $30 \mathrm{~min}$. The reactions were then diluted with $\mathrm{Et}_{2} \mathrm{O}(5 \mathrm{~mL}$ for each vial) and poured into a separatory funnel. The mixture was then washed with $1 \mathrm{M} \mathrm{HCl}(30 \mathrm{~mL})$ and the organic layer was separated. The aqueous layer was washed with $\operatorname{Et}_{2} \mathrm{O}(2 \times 15 \mathrm{~mL})$. The combined organic layers were then dried over $\mathrm{MgSO}_{4}$, filtered, and concentrated to. The crude residue was purified by flash column chromatography $\left(\mathrm{SiO}_{2}, 1: 0 \rightarrow 9: 1 \mathrm{Hex} / \mathrm{EtOAc}\right)$ provided ketone 6 (143 mg, 57\%) as a colorless oil.

The spectroscopic properties of the product matched those reported above. 


\section{Benesi-Hildebrand Analysis of NHPI Ester-HE EDA Complex}

The association constant $\left(K_{a}\right)$ of the EDA complex formed between adamantyl NHPI ester and HE was determined using the Benesi-Hildebrand method. ${ }^{26}$ In a quartz cuvette, the absorbance of a freshly prepared solution of adamantyl NHPI ester $(50 \mathrm{mM})$ in THF was measured using a Jasco V-530 UV/Vis spectrophotometer. To the solution of NHPI ester was then added 0.5 equiv of HE. The absorbance of this solution was then measured, and the procedure was repeated to give a range of 0.5 to 3.0 equivalents of HE compared to the concentration of NHPI ester.

In order to analyze the data using the Benesi-Hildebrand method, we then plotted the reciprocal of the change of absorbance of the EDA complex at $450 \mathrm{~nm}(\Delta A 450 \mathrm{~nm})$ versus the reciprocal of the concentration of HE. The corresponding plot can be found below in Figure S3.

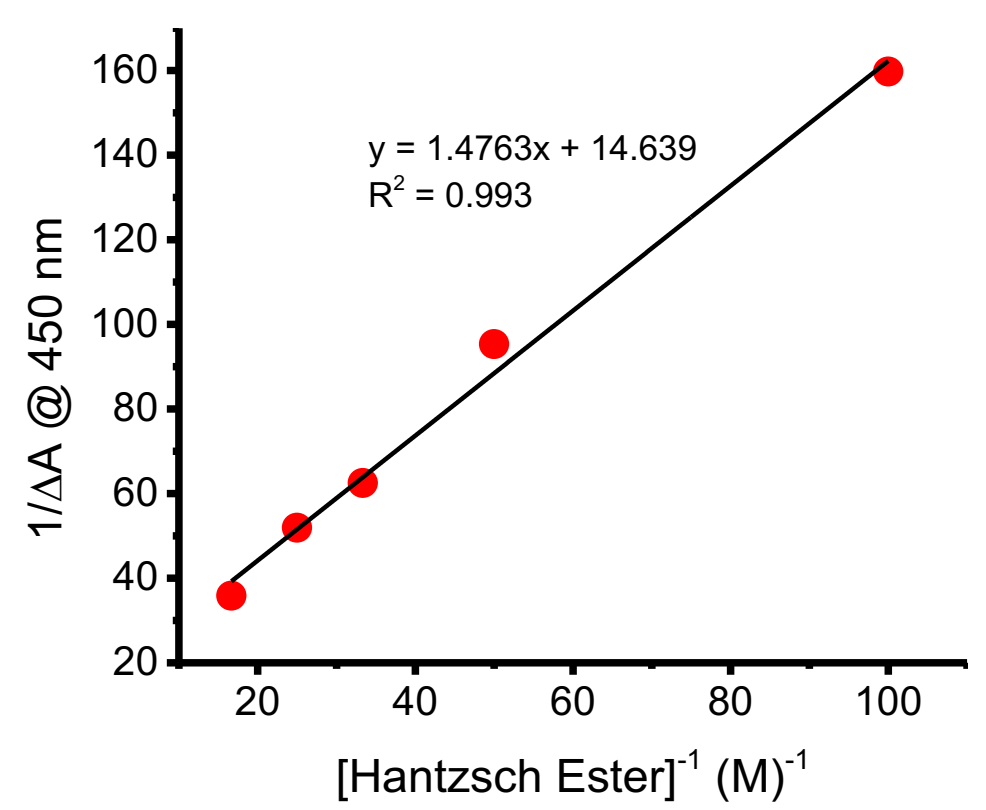

Figure S3. Benesi-Hildebrand plot for the determination of $K_{a}$ between HE and adamantyl NHPI ester. The change in absorbance at $450 \mathrm{~nm}$ was monitored as increasing concentrations of HE (0.53.0 equiv) was added to a solution of adamantyl NHPI ester $(50 \mathrm{mM})$ in THF.

The linear relationship was then used to solve for the Benesi-Hildebrand equation (eq 14), giving a $K_{a}$ of $9.9 \mathrm{M}^{-1}$ in THF.

$$
\begin{gathered}
\frac{1}{\Delta A_{450 \mathrm{~nm}}}=\frac{1}{A_{450 \mathrm{~nm}}} \times \frac{1}{K_{a}} \frac{1}{[H E]}+\frac{1}{A_{450 \mathrm{~nm}}} \\
\frac{1}{A_{450 \mathrm{~nm}}}=14.639 \\
14.639 \times \frac{1}{K_{a}}=1.4763 \\
K_{a}=9.9 \mathrm{M}^{-1}
\end{gathered}
$$


In order to study the ternary complex, the Benesi-Hildebrand analysis was repeated by adding increasing concentrations of HE to a THF solution containing the NHPI ester with 0.5 equiv of $\mathrm{Gd}(\mathrm{OTf})_{3}$. This stoichiometry was chosen for initial studies to simulate the behavior of the system under typical reaction stoichiometries. By employing the same analysis as previously described, we calculated that the $K_{a}$ for the formation of the EDA complex was $60 \mathrm{M}^{-1}$ (Figure S4), around six-fold higher compared to our previous result in the absence of $\mathrm{Gd}(\mathrm{OTf})_{3}$. This result supports our experimental observations that the rate of radical formation, which takes place through the excitation of the EDA complex, is enhanced in the presence of $\operatorname{Gd}(\mathrm{OTf})_{3}$.

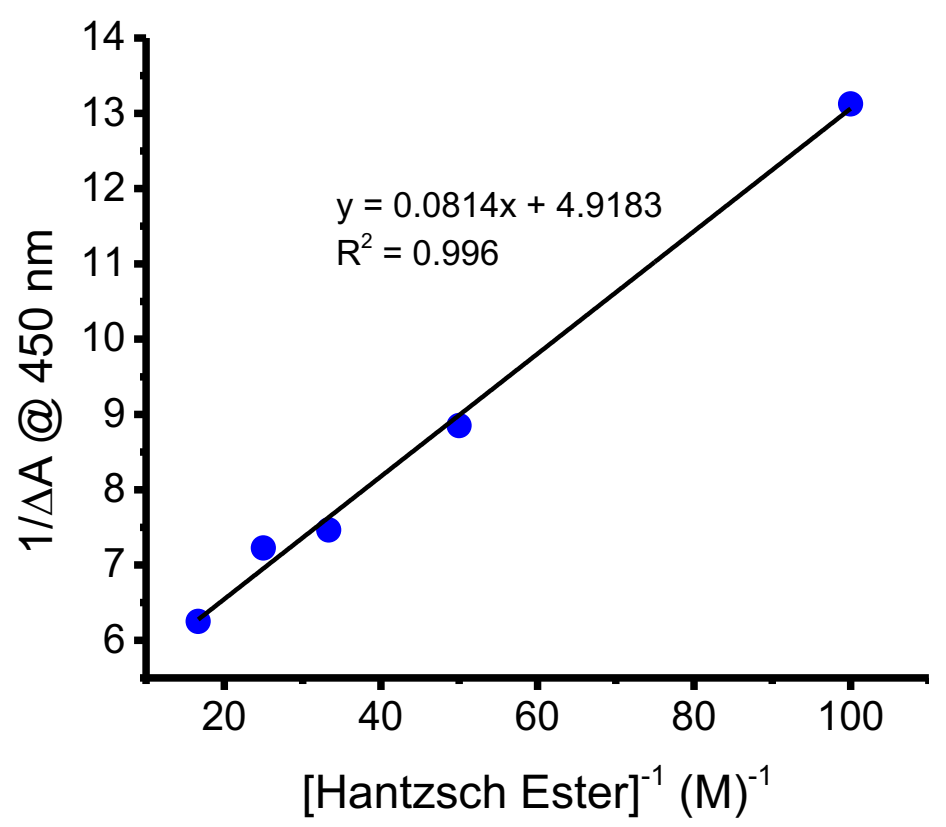

Figure S4. Benesi-Hildebrand plot for the determination of $K_{a}$ between HE and adamantyl NHPI ester in the presence of $\mathrm{Gd}(\mathrm{OTf})_{3}$. The change in absorbance at $450 \mathrm{~nm}$ was monitored as increasing concentrations of HE (0.5-3.0 equiv) was added to a solution of adamantyl NHPI ester $(50 \mathrm{mM})$ and $\mathrm{Gd}(\mathrm{OTf})_{3}(25 \mathrm{mM})$ in THF. 


\section{Conversion vs. Time Experiments}<smiles>CO[C@]1(C)O[C@H]2C=CC(=O)C[C@H]2O[C@]1(C)O</smiles>

35

$24 \mathrm{mg}$

$0.1 \mathrm{mmol}$<smiles>CC1(C(=O)ON2C(=O)c3ccccc3C2=O)CCCCC1</smiles>

4

$43 \mathrm{mg}$

$0.15 \mathrm{mmol}$<smiles>CCOC(=O)C1=C(C(=O)OCC)C([N+](=O)[O-])NC([N+](=O)[O-])=C1</smiles>

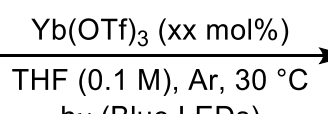
hv (Blue LEDs)

HE

$33 \mathrm{mg}$

$0.13 \mathrm{mmol}$

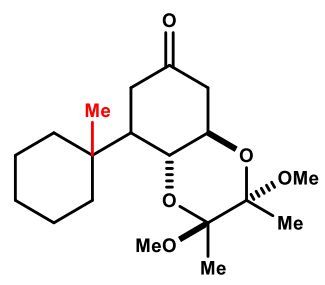

20

$\sim 4: 1 d r$

Flame-dried 1-dram vials were charged with the above listed amounts of the enone 35, NHPI ester 4 and $\mathrm{HE}$. Varying amounts of $\mathrm{Yb}(\mathrm{OTf})_{3}$ were added to each vial depending on loading being examined. The mixtures were then taken up in THF $(1 \mathrm{~mL}, 0.1 \mathrm{M})$, sparged with argon for $5 \mathrm{~min}$, and sonicated for 30 seconds. The reactions were then irradiated with two $34 \mathrm{~W}$ blue LEDs for the allotted time. Each reaction was then diluted with $\mathrm{Et}_{2} \mathrm{O}(10 \mathrm{~mL})$ and washed with $\mathrm{H}_{2} \mathrm{O}(10 \mathrm{~mL})$. The combined organic layers were dried over $\mathrm{MgSO}_{4}$, filtered, and concentrated under reduced pressure. Each time point reaction utilized $9.3 \mathrm{mg}$ of 1,2-dibromo-4,5-methylenedioxybenzene as an external standard. Yields and conversion were determined by ${ }^{1} \mathrm{H}$ NMR analysis of the crude NMR of each reaction. The methyl protons of the ketone product $\mathbf{2 0}$ and the $\beta$-proton of enone $\mathbf{3 5}$ were used to estimate yield and conversion, respectively.

Table S3. Amount of ketone product 20 formed in mmol at different $\left[\mathrm{Yb}(\mathrm{OTf})_{3}\right]$ as a function of time.

\begin{tabular}{|c|c|c|c|c|c|}
\hline \multicolumn{5}{|c|}{$\left[\mathbf{Y b}(\mathbf{O T f})_{3}\right]$} \\
\hline Time (min) & $\mathbf{1 0} \mathbf{~ m M}$ & $\mathbf{2 0} \mathbf{~ m M}$ & $\mathbf{3 0} \mathbf{~ m M}$ & $\mathbf{4 0} \mathbf{~ m M}$ & $\mathbf{5 0} \mathbf{~ m M}$ \\
\hline 0 & 0 & 0 & 0 & 0 & 0 \\
\hline 2 & 0.002 & 0.006 & 0.009 & 0.014 & 0.015 \\
\hline 5 & 0.005 & 0.01 & 0.021 & 0.027 & 0.028 \\
\hline 10 & 0.011 & 0.016 & 0.025 & 0.035 & 0.047 \\
\hline 15 & 0.016 & 0.021 & 0.038 & 0.047 & 0.053 \\
\hline 20 & 0.02 & 0.034 & 0.042 & 0.053 & 0.055 \\
\hline 25 & 0.02 & 0.039 & 0.045 & 0.055 & 0.056 \\
\hline 30 & 0.021 & 0.042 & 0.050 & 0.056 & 0.062 \\
\hline
\end{tabular}


Table S4. Amount of enone 35 remaining in mmol at different $\left[\mathrm{Yb}(\mathrm{OTf})_{3}\right]$ as a function of time.

\begin{tabular}{|c|c|c|c|c|c|}
\hline & \multicolumn{5}{|c|}{$\left[\mathbf{Y b}(\mathbf{O T f})_{3}\right]$} \\
\hline Time (min) & $\mathbf{1 0} \mathbf{~ m M}$ & $\mathbf{2 0} \mathbf{~ m M}$ & $\mathbf{3 0} \mathbf{~ m M}$ & $\mathbf{4 0} \mathbf{~ m M}$ & $\mathbf{5 0} \mathbf{~ m M}$ \\
\hline 0 & 0.1 & 0.1 & 0.1 & 0.1 & 0.1 \\
\hline 2 & 0.098 & 0.094 & 0.088 & 0.080 & 0.079 \\
\hline 5 & 0.094 & 0.09 & 0.07 & 0.064 & 0.067 \\
\hline 10 & 0.088 & 0.08 & 0.06 & 0.052 & 0.044 \\
\hline 15 & 0.073 & 0.073 & 0.045 & 0.045 & 0.039 \\
\hline 20 & 0.067 & 0.057 & 0.043 & 0.04 & 0.035 \\
\hline 25 & 0.061 & 0.053 & 0.041 & 0.035 & 0.034 \\
\hline 30 & 0.06 & 0.048 & 0.038 & 0.03 & 0.023 \\
\hline
\end{tabular}

Table S5. Amount of NHPI ester 4 remaining in mmol at different $\left[\mathrm{Yb}(\mathrm{OTf})_{3}\right]$ as a function of time.

\begin{tabular}{|c|c|c|c|c|c|}
\hline & \multicolumn{5}{|c|}{$\left[\mathbf{Y b}(\mathbf{O T f})_{\mathbf{3}}\right]$} \\
\hline Time (min) & $\mathbf{1 0} \mathbf{~ m M}$ & $\mathbf{2 0} \mathbf{~ m M}$ & $\mathbf{3 0} \mathbf{~ m M}$ & $\mathbf{4 0} \mathbf{~ m M}$ & $\mathbf{5 0} \mathbf{~ m M}$ \\
\hline 0 & 0.15 & 0.15 & 0.15 & 0.15 & 0.15 \\
\hline 2 & 0.145 & 0.142 & 0.138 & 0.136 & 0.135 \\
\hline 5 & 0.137 & 0.131 & 0.128 & 0.12 & 0.12 \\
\hline 10 & 0.129 & 0.119 & 0.115 & 0.095 & 0.087 \\
\hline 15 & 0.122 & 0.108 & 0.093 & 0.075 & 0.075 \\
\hline 20 & 0.114 & 0.105 & 0.084 & 0.071 & 0.063 \\
\hline 25 & 0.106 & 0.085 & 0.074 & 0.059 & 0.049 \\
\hline 30 & 0.095 & 0.071 & 0.066 & 0.054 & 0.042 \\
\hline
\end{tabular}

Table S6. Calculated initial rates of ketone product $\mathbf{2 0}$ formation as a function of $\left[\mathrm{Yb}(\mathrm{OTf})_{3}\right]$ after $\mathrm{t}=5$ mins.

\begin{tabular}{|c|c|}
\hline$\left[\mathbf{Y b}(\mathbf{O T f})_{3}\right] \mathbf{~ ( m M )}$ & Initial Rate (mmol/min) \\
\hline 10 & 0.001 \\
\hline 20 & 0.002 \\
\hline 30 & 0.0042 \\
\hline 40 & 0.0054 \\
\hline 50 & 0.0056 \\
\hline
\end{tabular}




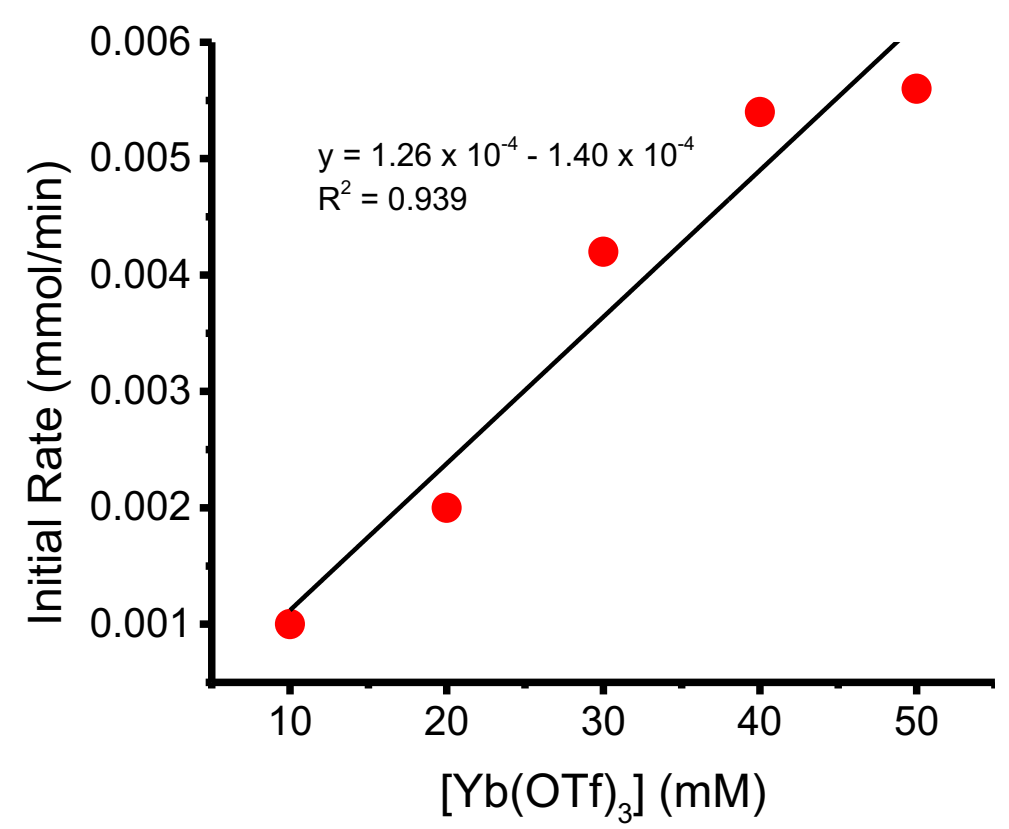

Figure S5. Plot of initial reaction rate of product formation vs. [Yb(OTf $\left.)_{3}\right]$.

Table S7. Calculated initial rates of conversion of enone $\mathbf{3 5}$ as a function of $\left[\mathrm{Yb}(\mathrm{OTf})_{3}\right]$ after $\mathrm{t}=$ 5 mins.

\begin{tabular}{|c|c|}
\hline$\left.\left[\mathbf{Y b}(\mathbf{O T f})_{3}\right] \mathbf{~ ( m M )}\right)$ & Initial Rate (mmol/min) \\
\hline 10 & 0.0012 \\
\hline 20 & 0.002 \\
\hline 30 & 0.006 \\
\hline 40 & 0.0072 \\
\hline 50 & 0.0066 \\
\hline
\end{tabular}

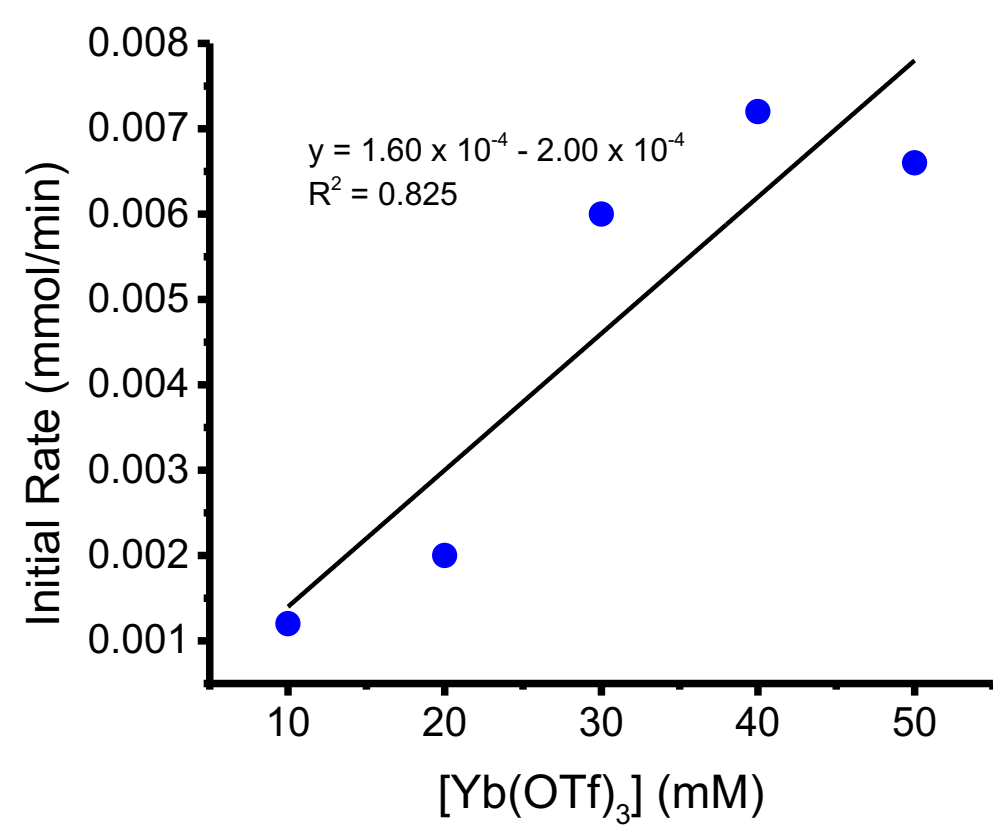

Figure S6. Plot of initial rate of conversion of enone $\mathbf{3 5} \mathrm{vs.}$ [ $\left.\mathrm{Yb}(\mathrm{OTf})_{3}\right]$. 
Table S8. Calculated initial rates of conversion of NHPI ester $\mathbf{4}$ as a function of $\left[\mathrm{Yb}(\mathrm{OTf})_{3}\right]$ after $\mathrm{t}=5$ mins.

\begin{tabular}{|c|c|}
\hline$[$ Yb(OTf) 3$]$ ( $\mathbf{m M})$ & Initial Rate (mmol/min) \\
\hline 10 & 0.0026 \\
\hline 20 & 0.0038 \\
\hline 30 & 0.0044 \\
\hline 40 & 0.006 \\
\hline 50 & 0.006 \\
\hline
\end{tabular}

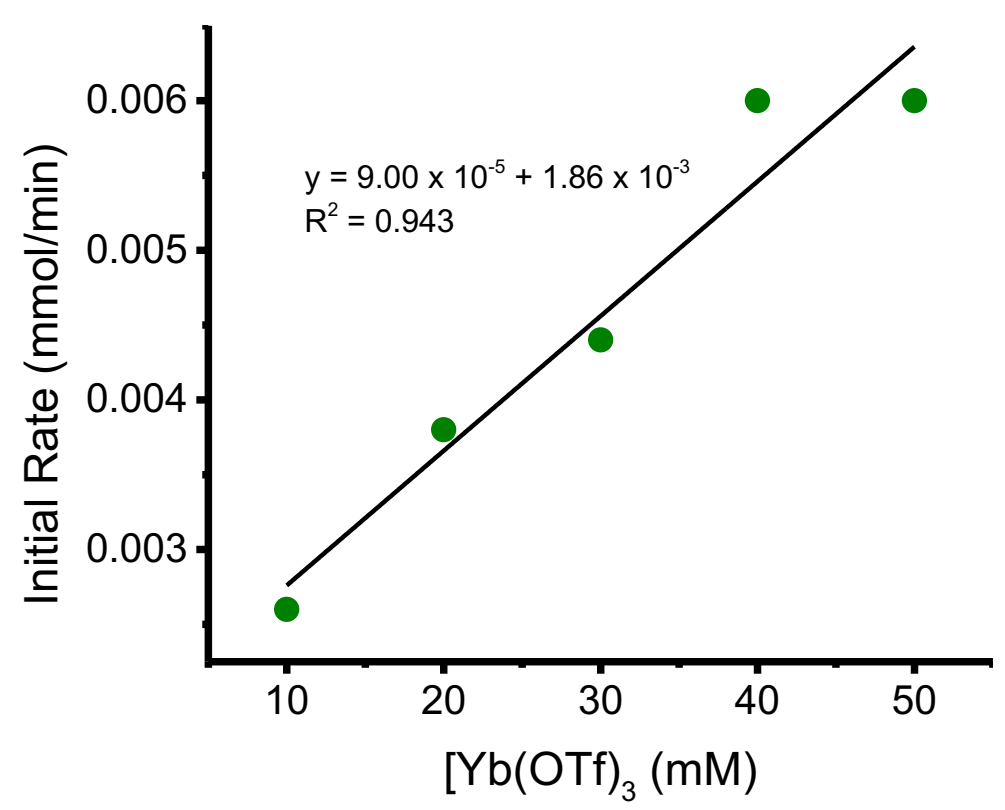

Figure S7. Plot of initial rate of conversion of NHPI ester 4 vs. [Yb(OTf $\left.)_{3}\right]$. 


\section{N. Unsuccessful or Low Yielding Scope Examples}

Table S9. Scope of unsuccessful or low yielding substrates for the Gd(OTf $)_{3}$-assisted Giesecoupling reaction. Potential reasons for low yield or failure are also included.
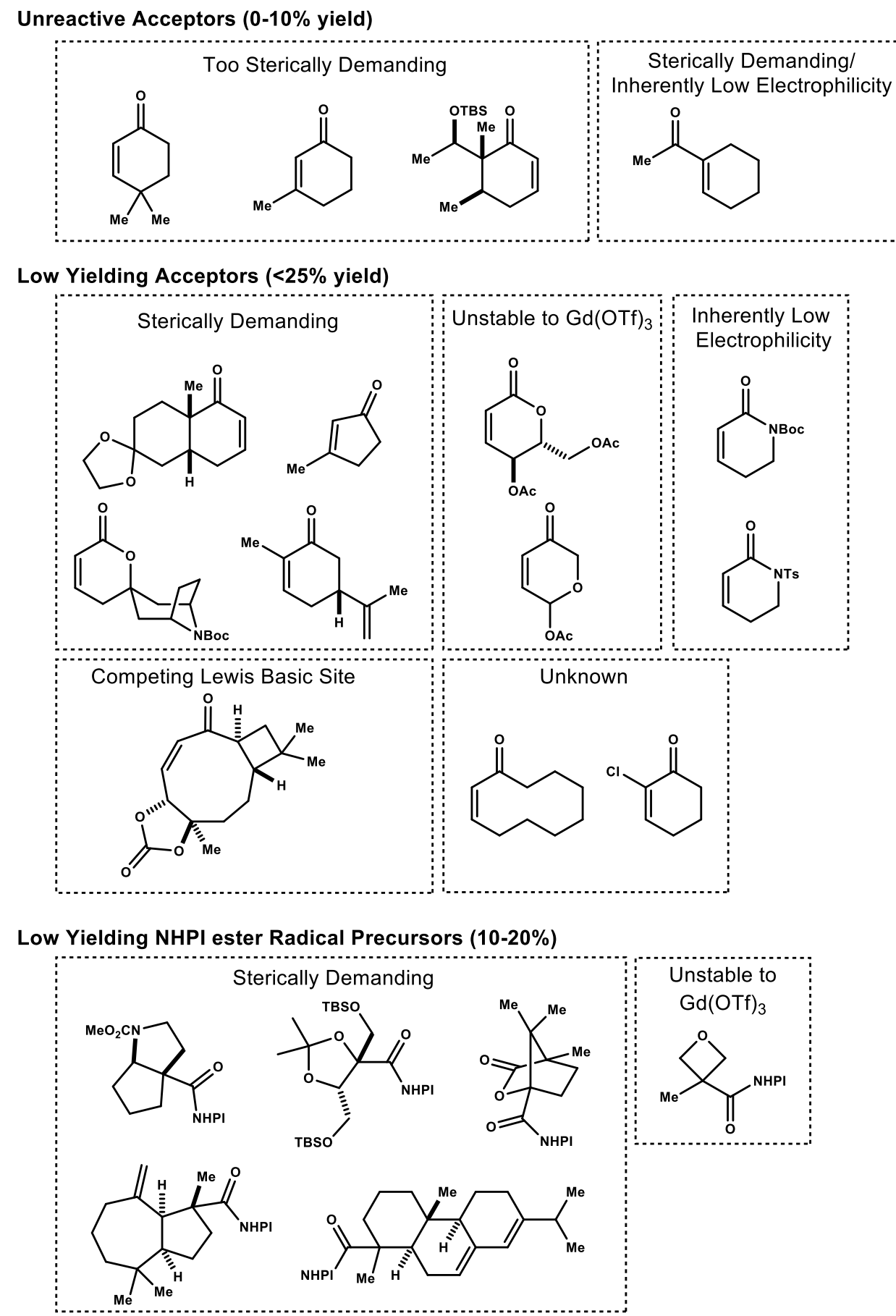


\section{O. Syntheses of Substrates that Coupled in Low Yield}

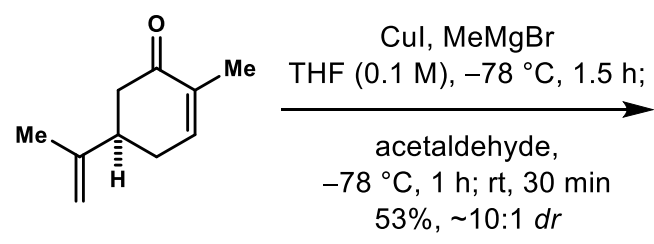

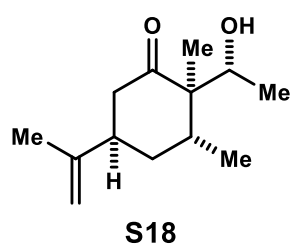

1. TBSOTf, 2,6-Lutidine, $\mathrm{CH}_{2} \mathrm{Cl}_{2}, 0^{\circ} \mathrm{C}, 16 \mathrm{~h}$

2. $\mathrm{O}_{3}, \mathrm{MeOH},-50^{\circ} \mathrm{C}$; $\mathrm{Cu}(\mathrm{OAc})_{2}, \mathrm{FeSO}_{4}$, $-30{ }^{\circ} \mathrm{C}$ to rt, $16 \mathrm{~h}$ $51 \%$ over 2 steps

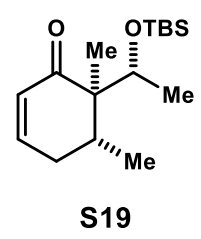

S19

$\boldsymbol{\beta}$-Hydroxy Ketone S18: The following was adapted from the conjugate addition/aldol procedure reported by Baran and coworkers. ${ }^{27}$ A flame-dried $100 \mathrm{~mL}$ round-bottom flask was charged with $\mathrm{CuI}(0.95 \mathrm{~g}, 4.98 \mathrm{mmol}, 1$ equiv) and THF $(50 \mathrm{~mL}, 0.1 \mathrm{M})$. The vigorously stirred suspension was then cooled to $-78^{\circ} \mathrm{C}$ and $\mathrm{MeMgBr}\left(3.0 \mathrm{M}\right.$ in $\mathrm{Et}_{2} \mathrm{O}, 3.7 \mathrm{~mL}, 11.1 \mathrm{mmol}, 2.2$ equiv) was added. The mixture was stirred at $-78^{\circ} \mathrm{C}$ for $30 \mathrm{~min}$, then warmed to $0{ }^{\circ} \mathrm{C}$ and stirred for $1 \mathrm{~h}$. The suspension changed in color from a light tan to yellow-brown. The mixture was then cooled to $-78{ }^{\circ} \mathrm{C}$ and $(R)$ - $(-)$-carvone $(0.78 \mathrm{~mL}, 4.98 \mathrm{mmol})$ was added dropwise over $5 \mathrm{~min}$. The mixture changed to a bright yellow color and was stirred for $1.5 \mathrm{~h}$ at $-78^{\circ} \mathrm{C}$. Then freshly distilled acetaldehyde $(0.57$ $\mathrm{mL}, 10 \mathrm{mmol}, 2$ equiv) was added dropwise. The mixture was stirred at $-78^{\circ} \mathrm{C}$ for $1 \mathrm{~h}$ and then warmed to rt over $30 \mathrm{~min}$. The reaction was then quenched with sat. aq. $\mathrm{NH}_{4} \mathrm{Cl}(30 \mathrm{~mL})$ and $2 \mathrm{M}$ aq. $\mathrm{NaOH}(20 \mathrm{~mL})$. The mixture was vigorously stirred at $\mathrm{rt}$ for $30 \mathrm{~min}$. The biphasic mixture was then poured into a separatory funnel containing sat. aq. $\mathrm{NH}_{4} \mathrm{Cl}(50 \mathrm{~mL})$ and was extracted with EtOAc $(50 \mathrm{~mL} \times 3)$. The combined organic layers were then dried over $\mathrm{MgSO}_{4}$, filtered, and concentrated under reduced pressure to afford an orange oil. The crude residue was purified by flash column chromatography $\left(\mathrm{SiO}_{2}, 95: 5 \rightarrow 10: 1 \mathrm{Hex} / \mathrm{EtOAc}\right)$ to afford pure alcohol S18 $(506.4$ $\mathrm{mg}, 48 \%)$ as a colorless oil and the minor diastereomer $(54.5 \mathrm{mg}, 5 \%)$. $\mathrm{R}_{\mathrm{f}}$ (major) $=0.30(4: 1$ $\mathrm{Hex} /$ EtOAc, visualized with $\mathrm{KMnO}_{4}$ ).

${ }^{1} \mathbf{H}$ NMR $\left(600 \mathrm{MHz}, \mathrm{CDCl}_{3}\right) \delta 4.85(\mathrm{~s}, 1 \mathrm{H}), 4.70(\mathrm{~s}, 1 \mathrm{H}), 3.98(\mathrm{q}, J=6.5 \mathrm{~Hz}, 1 \mathrm{H}), 2.62(\operatorname{app} \mathrm{p}, 6.4$ $\mathrm{Hz}, 1 \mathrm{H}), 2.51(\mathrm{dd}, J=5.3,14.6 \mathrm{~Hz}, 1 \mathrm{H}), 2.40(\mathrm{dd}, J=7.9,14.6 \mathrm{~Hz}, 1 \mathrm{H}), 2.27-2.20(\mathrm{~m}, 1 \mathrm{H}), 1.98-$ $1.91(\mathrm{~m}, 1 \mathrm{H}), 1.75(\mathrm{~s}, 3 \mathrm{H}), 1.74-1.70(\mathrm{~m}, 1 \mathrm{H}), 1.59$ (br s, $1 \mathrm{H}), 1.14(\mathrm{~d}, J=6.5 \mathrm{~Hz}, 3 \mathrm{H}), 1.13(\mathrm{~s}$, $3 \mathrm{H}), 0.89(\mathrm{~d}, J=7.0 \mathrm{~Hz}, 3 \mathrm{H})$

${ }^{13}$ C NMR $\left(150 \mathrm{MHz}, \mathrm{CDCl}_{3}\right) \delta 217.5,147.3,111.1,70.8,56.0,43.7,40.4,33.1,32.3,21.4,18.7$, $15.3,14.2$

IR (thin film, $\mathrm{cm}^{-1}$ ) 3445, 2935, 1693, 1644, 1454, 1381, 1283, 1219, 1150, 1092

HRMS (ESI-TOF) m/z: [M+Na $]^{+}$calcd for $\mathrm{C}_{13} \mathrm{H}_{22} \mathrm{O}_{2} \mathrm{Na} 233.1517$, found 233.1521

Optical Rotation: $[\alpha]^{21.6} \mathrm{D}+0.6,[\alpha]^{21.8}{ }_{577}+0.5,[\alpha]^{21.8}{ }_{546}+1.9,[\alpha]^{21.9}{ }_{435}+25.3\left(c=1.0, \mathrm{CHCl}_{3}\right)$

Enone S19: To a solution of alcohol $\mathbf{S 1 8}(421 \mathrm{mg}, 2.0 \mathrm{mmol})$ in $\mathrm{CH}_{2} \mathrm{Cl}_{2}(12 \mathrm{~mL}, 0.15 \mathrm{M})$ at $0{ }^{\circ} \mathrm{C}$, 2,6-lutidine ( $0.95 \mathrm{~mL}, 8.2 \mathrm{mmol}, 4.1$ equiv) and TBSOTf (0.94 mL, $4.1 \mathrm{mmol}, 2.0$ equiv) were added. The mixture was then allowed to warm to $\mathrm{rt}$ and stirred for $3 \mathrm{~h}$. The reaction was then quenched with $\mathrm{MeOH}(0.5 \mathrm{~mL})$ and stirred for $20 \mathrm{~min}$. The reaction was then diluted with $1 \mathrm{M}$ aq. $\mathrm{HCl}(20 \mathrm{~mL})$ and extracted with $\mathrm{CH}_{2} \mathrm{Cl}_{2}(2 \times 30 \mathrm{~mL})$. The combined organic layers were then dried over $\mathrm{MgSO}_{4}$, filtered, and concentrated to afford the crude silyl ether, which was used without further purification. 
This crude product was dissolved in $\mathrm{MeOH}(20 \mathrm{~mL}, 0.1 \mathrm{M})$ and cooled to $-50{ }^{\circ} \mathrm{C}$. Then $\mathrm{O}_{3}$ was bubbled through the solution until a pale blue color persisted. The solution was then sparged with $\mathrm{O}_{2}$ until the color dissipated. The reaction was then sparged with argon for $15 \mathrm{~min}$. The reaction was then warmed to $-30{ }^{\circ} \mathrm{C}$ and $\mathrm{Cu}(\mathrm{OAc})_{2}(641 \mathrm{mg}, 3.21 \mathrm{mmol}, 1.6$ equiv) was added. The reaction was vigorously stirred for $10 \mathrm{~min}$ and then $\mathrm{FeSO}_{4}(567 \mathrm{mg}, 2.04 \mathrm{mmol}, 1$ equiv) was added. The reaction was vigorously stirred and allowed to slowly warm to rt overnight ( $\sim 16 \mathrm{~h})$. The reaction was then diluted with $\mathrm{H}_{2} \mathrm{O}(100 \mathrm{~mL})$ and extracted with $\mathrm{Et}_{2} \mathrm{O}(5 \times 30 \mathrm{~mL})$. The combined organic layers were then dried over $\mathrm{MgSO}_{4}$, filtered, and concentrated. The crude residue was purified by flash column chromatography $\left(\mathrm{SiO}_{2}, 1: 0 \rightarrow 9: 1 \mathrm{Hex} / \mathrm{EtOAc}\right)$ to afford enone S19 (288 mg, 51\% yield over two steps) as a white solid. $\mathrm{R}_{\mathrm{f}}=0.53$ (9:1 Hex/EtOAc, visualized with $\mathrm{KMnO}_{4}$ stain).

${ }^{1} \mathbf{H}$ NMR $\left(600 \mathrm{MHz}, \mathrm{CDCl}_{3}\right) \delta 6.78-6.72(\mathrm{~m}, 1 \mathrm{H}), 5.91(\mathrm{dd}, J=1.9,10.0 \mathrm{~Hz}, 1 \mathrm{H}), 4.21(\mathrm{q}, J=6.3$ $\mathrm{Hz}, 1 \mathrm{H}), 2.82-2.73(\mathrm{~m}, 1 \mathrm{H}), 2.49(\mathrm{p}, J=6.8 \mathrm{~Hz}, 1 \mathrm{H}), 2.10-2.02(\mathrm{~m}, 1 \mathrm{H}), 1.01(\mathrm{~d}, J=6.3 \mathrm{~Hz}, 3 \mathrm{H})$, $0.97(\mathrm{~s}, 3 \mathrm{H}), 0.96(\mathrm{~d}, J=6.0 \mathrm{~Hz}, 3 \mathrm{H}), 0.87(\mathrm{~s}, 9 \mathrm{H}), 0.06(\mathrm{~s}, 3 \mathrm{H}), 0.04(\mathrm{~s}, 3 \mathrm{H})$

${ }^{13} \mathrm{C}$ NMR (150 MHz, $\left.\mathrm{CDCl}_{3}\right) \delta 203.9,147.0,129.2,70.0,55.3,34.0,31.7,25.8,19.5,18.1,16.6$, $13.2,-3.8,-4.9$

IR (thin film, $\mathrm{cm}^{-1}$ ) 2955, 2928, 2856, 1677, 1462, 1386, 1250, 1219, 1106

HRMS (ESI-TOF) m/z: [M+H] $]^{+}$calcd for $\mathrm{C}_{16} \mathrm{H}_{31} \mathrm{O}_{2} \mathrm{Si} 283.2093$, found 283.2094

Optical Rotation: $[\alpha]^{21.7} \mathrm{D}+15.2,[\alpha]^{21.8}{ }_{577}+15.1,[\alpha]^{21.9}{ }_{546}+14.9,[\alpha]^{21.9}{ }_{435}+11.9\left(c=1.0, \mathrm{CHCl}_{3}\right)$

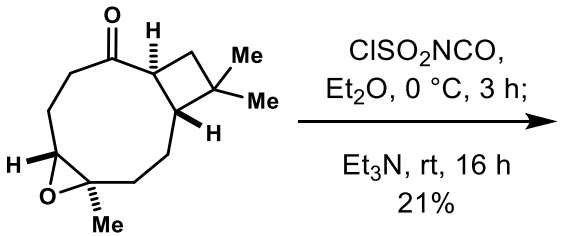
in $\mathrm{Et}_{2} \mathrm{O}(18 \mathrm{~mL}, 0.3 \mathrm{M})$ and cooled to $0{ }^{\circ} \mathrm{C}$. Then chlorosulfonyl isocyanate ${ }^{29}(0.93 \mathrm{~mL}, 11 \mathrm{mmol}$, 2 equiv) was added dropwise. The reaction was stirred for $3 \mathrm{~h}$ while warming to rt. TLC indicated that the starting material had been consumed. Then $\mathrm{Et}_{3} \mathrm{~N}(5.2 \mathrm{~mL}, 37.1 \mathrm{mmol}, 7$ equiv $)$ was added slowly resulting in a partitioning of the reaction into a yellow viscous bottom layer and an opaque colorless layer on top. The mixture was vigorously stirred for $20 \mathrm{~min}$ to ensure adequate mixing of the two layers. Then $\mathrm{H}_{2} \mathrm{O}(20 \mathrm{~mL})$ was added and the mixture was vigorously stirred overnight. The reaction was then extracted with EtOAc $(2 \times 30 \mathrm{~mL})$. The combined organic layers was then dried over $\mathrm{MgSO}_{4}$, filtered, and concentrated to a crude yellow solid. This residue was purified by flash column chromatography $\left(\mathrm{SiO}_{2}, 1: 0 \rightarrow 4: 1 \rightarrow 2: 1 \rightarrow 1: 1 \mathrm{Hex} / \mathrm{EtOAc}\right)$ to afford carbonate $\mathbf{S 2 0}$ (299 $\mathrm{mg}, 21 \%$ ) as a colorless solid. $\mathrm{R}_{\mathrm{f}}=0.36$ (2:1 Hex/EtOAc, visualized with CAM stain). 
${ }^{1} \mathbf{H}$ NMR $\left(600 \mathrm{MHz}, \mathrm{CDCl}_{3}\right) \delta 4.11$ (app t, $J=6.0 \mathrm{~Hz}, 1 \mathrm{H}$ ), 3.09 (app q, $\left.J=8.8 \mathrm{~Hz}, 1 \mathrm{H}\right), 2.58-$ $2.52(\mathrm{~m}, 1 \mathrm{H}), 2.50-2.44(\mathrm{~m}, 1 \mathrm{H}), 2.28-2.05(\mathrm{~m}, 4 \mathrm{H}), 1.93-1.78(\mathrm{~m}, 3 \mathrm{H}), 1.47(\mathrm{~d}, J=7.6 \mathrm{~Hz}, 1 \mathrm{H})$, $1.46(\mathrm{~d}, J=7.6 \mathrm{~Hz}, 1 \mathrm{H}), 1.43(\mathrm{~s}, 3 \mathrm{H}), 1.02(\mathrm{~s}, 6 \mathrm{H})$

${ }^{13} \mathrm{C}$ NMR $\left(150 \mathrm{MHz}, \mathrm{CDCl}_{3}\right) \delta 211.3,153.5,86.6,82.1,53.6,48.5,40.0,39.4,35.4,32.2,29.3$, $24.7,23.4,22.3,19.1$

IR (thin film, cm ${ }^{-1}$ ) 2949, 1796, 1696, 1453, 1373, 1253, 1227, 1187, 1060, 1042, 1015

HRMS (ESI-TOF) m/z: [M+NH 4$]^{+}$calcd for $\mathrm{C}_{15} \mathrm{H}_{26} \mathrm{NO}_{4} 284.1862$, found 284.1866

Optical Rotation: $[\alpha]^{21.8} \mathrm{D}-29.2,[\alpha]^{21.8}{ }_{577}-30.6,[\alpha]^{22.0_{546}}-34.7,[\alpha]^{22.0}{ }_{435}-45.3\left(c=1.0, \mathrm{CHCl}_{3}\right)$

Enone S21: To a solution of ketone $\mathbf{S 2 0}(293 \mathrm{mg}, 1.09 \mathrm{mmol})$ in THF $(11 \mathrm{~mL}, 0.1 \mathrm{M})$ at $-78^{\circ} \mathrm{C}$, LiHMDS (1.3 mL, $1 \mathrm{M}$ in THF, $1.3 \mathrm{mmol}, 1.2$ equiv) was added dropwise. The reaction was stirred at $-78{ }^{\circ} \mathrm{C}$ for $1 \mathrm{~h}$. Then freshly distilled TMSCl $(0.21 \mathrm{~mL}, 1.7 \mathrm{mmol}, 1.5$ equiv) was added dropwise. The reaction was stirred at $-78^{\circ} \mathrm{C}$ for $1 \mathrm{~h}$ then warmed to $0{ }^{\circ} \mathrm{C}$ for $2 \mathrm{~h}$. The reaction was then diluted with pentane $(30 \mathrm{~mL})$ and washed with $\mathrm{H}_{2} \mathrm{O}(50 \mathrm{~mL})$. The aqueous layer was washed with pentane $(2 \times 20 \mathrm{~mL})$. The combined organic layers were dried over $\mathrm{MgSO}_{4}$, filtered, and concentrated to a colorless oil, which was used without further purification.

This crude silyl enol ether was dissolved in DMSO (2.7 mL, 0.4 M) and IBX (461 mg, 1.65 mmol, 1.5 equiv) and 4-methoxypyridine $\mathrm{N}$-oxide (183 mg, $1.67 \mathrm{mmol}, 1.5$ equiv) were added simultaneously. The reaction was then stirred at $\mathrm{rt}$ for $16 \mathrm{~h}$. The reaction was then diluted with $\mathrm{H}_{2} \mathrm{O}(40 \mathrm{~mL})$ and extracted with EtOAc $(2 \times 20 \mathrm{~mL})$. The combined organic layers were then dried over $\mathrm{MgSO}_{4}$, filtered, and concentrated to yellow solids. The residue was purified by flash column chromatography $\left(\mathrm{SiO}_{2}, 1: 0 \rightarrow 2: 1 \mathrm{Hex} / \mathrm{EtOAc}\right)$ to afford enone $\mathbf{S 2 1}(172 \mathrm{mg}, 59 \%)$ as a colorless solid, as well as starting carbonate $\mathbf{S 2 0}(90.6 \mathrm{mg}, 31 \%)$. $\mathrm{R}_{\mathrm{f}}=0.52(2: 1 \mathrm{Hex} / \mathrm{EtOAc}$, visualized with CAM stain).

${ }^{1} \mathbf{H}$ NMR $\left(600 \mathrm{MHz}, \mathrm{CDCl}_{3}\right) \delta 6.33(\mathrm{~d}, J=12.3 \mathrm{~Hz}, 1 \mathrm{H}), 5.69(\mathrm{dd}, J=12.3,9.4 \mathrm{~Hz}, 1 \mathrm{H}), 4.82(\mathrm{~d}$, $J=9.4 \mathrm{~Hz}, 1 \mathrm{H}), 3.02(\mathrm{q}, J=8.7 \mathrm{~Hz}, 1 \mathrm{H}), 2.25-2.13(\mathrm{~m}, 2 \mathrm{H}), 1.98-1.78(\mathrm{~m}, 4 \mathrm{H}), 1.50(\mathrm{~d}, J=7.6$ $\mathrm{Hz}, 1 \mathrm{H}), 1.48(\mathrm{~d}, J=7.6 \mathrm{~Hz}, 1 \mathrm{H}), 1.42(\mathrm{~s}, 3 \mathrm{H}), 1.04(\mathrm{~s}, 3 \mathrm{H}), 1.02(\mathrm{~s}, 3 \mathrm{H})$

${ }^{13}$ C NMR (125 MHz, $\left.\mathrm{CDCl}_{3}\right) \delta 206.3,153.0,137.6,124.4,86.3,81.1,52.6,49.7,41.2,34.4,31.8$, $29.2,24.8,22.0,20.7$

IR (thin film, $\mathrm{cm}^{-1}$ ) 2948, 1796, 1690, 1457, 1403, 1334, 1244, 1227, 1124, 1074, 1062, 1042

HRMS (ESI-TOF) m/z: [M+NH 4$]^{+}$calcd for $\mathrm{C}_{15} \mathrm{H}_{24} \mathrm{NO}_{4} 282.1705$, found 282.1699

Optical Rotation: $[\alpha]^{21.7} \mathrm{D}+24.5,[\alpha]^{21.9}{ }_{577}+27.1,[\alpha]^{22.0}{ }_{546}+36.5,[\alpha]^{22.0}{ }_{435}+132\left(c=1.0, \mathrm{CHCl}_{3}\right)$ 


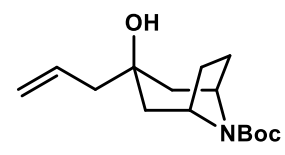

S22

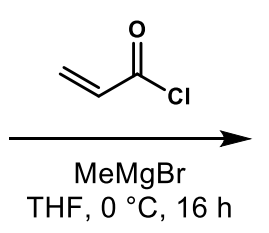

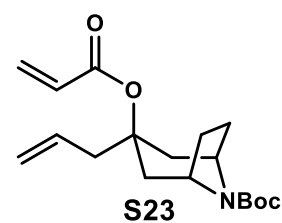

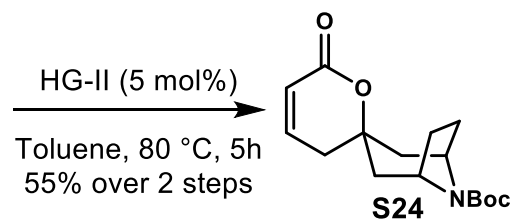

Spirolactone S24: A $100 \mathrm{~mL}$ round-bottom flask was charged with homoallylic alcohol S22 $^{30}$ $(1.52 \mathrm{~g}, 5.71 \mathrm{mmol})$ in THF $(29 \mathrm{~mL}, 0.2 \mathrm{M})$ and cooled to $0{ }^{\circ} \mathrm{C}$. $\mathrm{MeMgBr}(2.2 \mathrm{~mL}, 6.6 \mathrm{mmol}, 1.2$ equiv, $3 \mathrm{M}$ in $\mathrm{Et}_{2} \mathrm{O}$ ) was added dropwise. The reaction mixture was then stirred for $1 \mathrm{~h}$ at $0{ }^{\circ} \mathrm{C}$ and progressed to a cloudy, colorless mixture. Acryloyl chloride $(0.93 \mathrm{~mL}, 11 \mathrm{mmol}, 2$ equiv) was then added dropwise over $5 \mathrm{~min}$. The reaction was then allowed to slowly warm to $\mathrm{rt}$ overnight. The resulting yellow reaction mixture was then quenched by the addition of sat. aq. $\mathrm{NaHCO}_{3}(50 \mathrm{~mL})$. The resulting mixture was vigorously stirred for $15 \mathrm{~min}$. Then the mixture was extracted with EtOAc $(20 \mathrm{~mL} \times 3)$. The combined organic layers were then dried over $\mathrm{MgSO}_{4}$, filtered, and concentrated to a pale-yellow oil, which was carried on without further purification.

Crude diene $\mathbf{S 2 3}$ was then dissolved in toluene $(280 \mathrm{~mL}, 0.02 \mathrm{M})$ and warmed to $80{ }^{\circ} \mathrm{C}$. A solution to Hoyveda-Grubbs $2^{\text {nd }}$ generation catalyst $(170 \mathrm{mg}, 0.271 \mathrm{mmol}, 5 \mathrm{~mol} \%)$ in toluene (20 $\mathrm{mL}$ ) was then added dropwise via syringe pump over $4 \mathrm{hrs}$. The reaction was stirred at $80{ }^{\circ} \mathrm{C}$ for an additional hour. TLC indicated the that starting material had been consumed. The reaction mixture was then concentrated to a brown oil and purified by flash column chromatography $\left(\mathrm{SiO}_{2}\right.$, $10: 1 \rightarrow 4: 1 \rightarrow 1: 1 \mathrm{Hex} /$ EtOAc) to afford spirolactone $\mathbf{S 2 4}$ (924 mg, 55\% over two steps) as a light brown solid. $\mathrm{R}_{\mathrm{f}}=0.44(1: 1 \mathrm{Hex} /$ EtOAc) .

${ }^{1} \mathbf{H}$ NMR $\left(600 \mathrm{MHz}, \mathrm{CDCl}_{3}\right) \delta 6.73(\mathrm{dt}, J=4.4,9.8 \mathrm{~Hz}, 1 \mathrm{H}), 6.01(\mathrm{~d}, J=9.8 \mathrm{~Hz}, 1 \mathrm{H}), 4.26-4.20$ (m, 2H), 2.31-2.27 (m, 2H), 2.21-2.12 (m, 4H), 1.99-1.92 (m, 2H), 1.91-1.85 (m, 2H), 1.45 (s, 9H)

${ }^{13} \mathrm{C}$ NMR $\left(150 \mathrm{MHz}, \mathrm{CDCl}_{3}\right) \delta 163.5,153.4,142.9,121.3,80.7,79.6,52.4,41.2,37.5,28.5,27.7$

IR (thin film, $\mathrm{cm}^{-1}$ ) 2974, 2921, 1715, 1689, 1382, 1366, 1334, 1254, 1169, 1101, 1021

MP: $106-108^{\circ} \mathrm{C}$

HRMS (ESI-TOF) m/z: [M+Na $]^{+}$calcd for $\mathrm{C}_{16} \mathrm{H}_{23} \mathrm{NO}_{4} \mathrm{Na} 316.1525$, found 316.1525

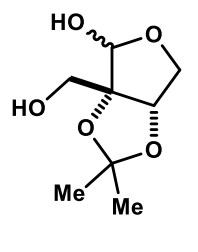

S25

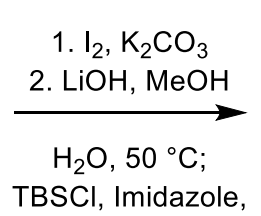

$\mathrm{CH}_{2} \mathrm{Cl}_{2}$

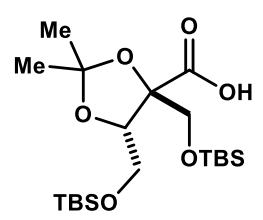

S26

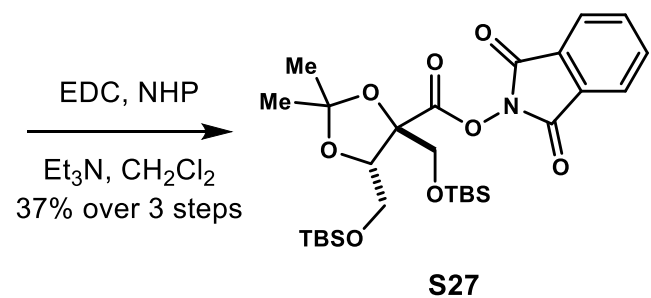

S27

Acetonide NHPI ester S27: A $100 \mathrm{~mL}$ round-bottom flask was charged with lactol $\mathbf{S 2 5}^{31}$ (1.57 g, $8.24 \mathrm{mmol})$ in $t$ - $\mathrm{BuOH}(27 \mathrm{~mL}, 0.3 \mathrm{M})$ and the solution was warmed to $75{ }^{\circ} \mathrm{C} . \mathrm{K}_{2} \mathrm{CO}_{3}(2.29 \mathrm{~g}$, $16.5 \mathrm{mmol}, 2$ equiv) and $\mathrm{I}_{2}(4.18 \mathrm{~g}, 16.5 \mathrm{mmol}, 2$ equiv) were added and the mixture was then heated to $100{ }^{\circ} \mathrm{C}$. After $2 \mathrm{~h}$, TLC indicated that the starting material had been consumed and the reaction was cooled to rt. Then sat. aq. $\mathrm{Na}_{2} \mathrm{~S}_{2} \mathrm{O}_{3}(20 \mathrm{~mL})$ was added followed by EtOAc $(20 \mathrm{~mL})$ 
and the mixture was stirred until the dark color dissipated. The reaction was extracted with EtOAc (30 $\mathrm{mL} \times 2$ ) and the combined organic layers were washed with brine, dried over $\mathrm{MgSO}_{4}$, filtered and concentrated to a yellow oily solid. This crude residue was suspended in 1:1 Hex/EtOAc (20 $\mathrm{mL})$ and flushed through $\mathrm{SiO}_{2}$ plug with 1:1 Hex/EtOAc $(200 \mathrm{~mL})$. The filtrate was concentrated to a colorless oily solid ( $855 \mathrm{mg}, 4.54 \mathrm{mmol}, 55 \%$ ), which was carried on without further purification.

Crude lactone was taken up in a 2:1 mixture of $\mathrm{MeOH}: \mathrm{H}_{2} \mathrm{O}(9 \mathrm{~mL}, 0.5 \mathrm{M})$ and $\mathrm{LiOH}(228$ $\mathrm{mg}, 5.44 \mathrm{mmol}, 1.2$ equiv) was added. The flask was sealed and the mixture was heated to $50{ }^{\circ} \mathrm{C}$ for $16 \mathrm{hr}$ until TLC indicated that the starting material had been consumed. The reaction was then concentrated under reduced pressure. The crude orange residue was then stripped with toluene (10 $\mathrm{mL} \times 3$ ) under reduced pressure affording the intermediate lithium carboxylate salt as yellow foam. The foam was suspended in $\mathrm{CH}_{2} \mathrm{Cl}_{2}(22 \mathrm{~mL}, 0.2 \mathrm{M})$ and imidazole (1.57 g, $23.0 \mathrm{mmol}, 5$ equiv) was added. The suspension was then vigorously stirred and $\mathrm{TBSCl}$ (2.29 g, $15.2 \mathrm{mmol}, 3.3 \mathrm{equiv})$ was added in three portions over $30 \mathrm{~min}$. The reaction was vigorously stirred for $24 \mathrm{hr}$, then $\mathrm{MeOH}$ $(2 \mathrm{~mL}$ ) was added and the mixture was stirred for $30 \mathrm{~min}$. The reaction was then diluted with $1 \mathrm{M}$ aq. $\mathrm{HCl}(30 \mathrm{~mL})$ and extracted with $\mathrm{CH}_{2} \mathrm{Cl}_{2}(30 \mathrm{~mL} \times 3)$. The combined organic layers were then dried over $\mathrm{MgSO}_{4}$, filtered and concentrated to a pale-yellow oil, which was carried on without further purification.

Crude acid $\mathbf{S 2 6}$ was then taken up in $\mathrm{CH}_{2} \mathrm{Cl}_{2}(22 \mathrm{~mL}, 0.2 \mathrm{M})$ and $\mathrm{EDC} \cdot \mathrm{HCl}(1.30 \mathrm{~g}, 6.80$ mmol, 1.5 equiv) was added followed by $\mathrm{Et}_{3} \mathrm{~N}(1.9 \mathrm{~mL}, 14 \mathrm{mmol}, 3$ equiv). The reaction was stirred for $5 \mathrm{~min}$ and then NHP (1.11 g, $6.80 \mathrm{mmol}, 1.5$ equiv) was added. The resulting red solution was stirred at rr for $20 \mathrm{hrs}$. The reaction was then diluted with aq. $1 \mathrm{M} \mathrm{HCl}(30 \mathrm{~mL})$ and extracted with $\mathrm{CH}_{2} \mathrm{Cl}_{2}(30 \mathrm{~mL} \times 3)$. The combined organic layers were then dried over $\mathrm{MgSO}_{4}$, filtered, and concentrated to a yellow oily solid. The crude residue was purified by flash column chromatography $\left(\mathrm{SiO}_{2}, 1: 0 \rightarrow 10: 1 \rightarrow 4: 1 \rightarrow 1: 1 \mathrm{Hex} /\right.$ EtOAc) to afford NHPI ester $\mathbf{S 2 7}(980 \mathrm{mg}, 37 \%$ over 3 steps $)$ as a clear oil that solidified upon storage at $-20^{\circ} \mathrm{C} . \mathrm{R}_{\mathrm{f}}=0.66(4: 1 \mathrm{Hex} / \mathrm{EtOAc})$

${ }^{1} \mathbf{H}$ NMR $\left(500 \mathrm{MHz}, \mathrm{CDCl}_{3}\right) \delta$ 7.91-7.85 (m, 2H), 7.80-7.74 (m, 2H), $4.46(\mathrm{t}, J=6.2 \mathrm{~Hz}, 1 \mathrm{H})$, 4.14-3.99 (m, 4H), $1.62(\mathrm{~s}, 3 \mathrm{H}), 1.48(\mathrm{~s}, 3 \mathrm{H}), 0.93(\mathrm{~s}, 9 \mathrm{H}), 0.90(\mathrm{~s}, 9 \mathrm{H}), 0.12(\mathrm{~s}, 6 \mathrm{H}), 0.10(\mathrm{~s}, 3 \mathrm{H})$, $0.09(\mathrm{~s}, 3 \mathrm{H})$

${ }^{13} \mathrm{C}$ NMR $\left(125 \mathrm{MHz}, \mathrm{CDCl}_{3}\right) \delta 167.5,161.5,134.7,129.1,124.0,111.4,86.2,79.3,64.2,62.2$, $26.6,26.4,26.0,25.9,18.5,18.3,-5.3,-5.35,-5.37,-5.5$

IR (thin film, $\mathrm{cm}^{-1}$ ) 2953, 2929, 2884, 2857, 1813, 1788, 1747, 1470, 1372, 1254, 1220, 1185 , $1100,1039,1005$

HRMS (ESI-TOF) m/z: [M+H] ${ }^{+}$calcd for $\mathrm{C}_{28} \mathrm{H}_{46} \mathrm{NO}_{8} \mathrm{Si}_{2}$ 580.2762, found 580.2761

Optical Rotation: $[\alpha]^{22.0}{ }_{\mathrm{D}}+17.8,[\alpha]^{22.0}{ }_{577}+18.7,[\alpha]^{22.0}{ }_{546}+20.2,[\alpha]^{22.0}{ }_{435}+33.3\left(c=1.0, \mathrm{CHCl}_{3}\right)$ 


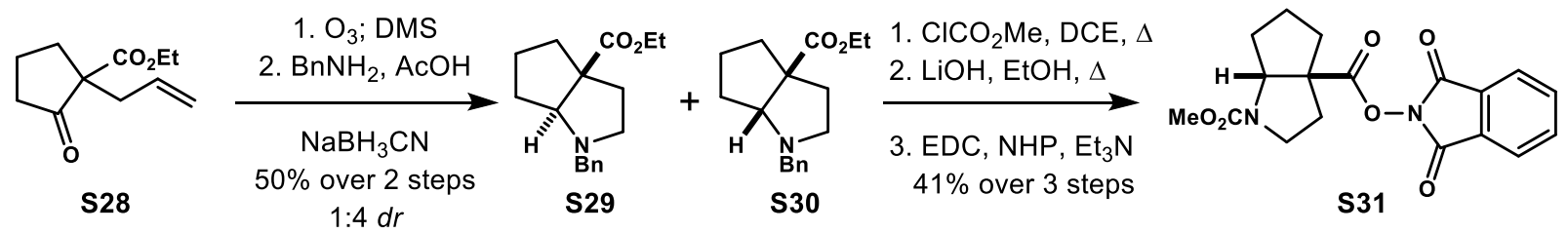

Azabicyclo[3.3.0]octanes S29 and S30: A $100 \mathrm{~mL}$ round-bottom flask was charged with olefin $\mathbf{S 2 8}^{32}(1.37 \mathrm{~g}, 6.99 \mathrm{mmol})$ in $\mathrm{CH}_{2} \mathrm{Cl}_{2}(35 \mathrm{~mL}, 0.2 \mathrm{M})$ and cooled to $-78{ }^{\circ} \mathrm{C}$. Ozone was bubbled through the mixture until the solution became a persistent blue color. Argon was then bubbled through the mixture until the blue color dissipated. Then dimethyl sulfide $(5.2 \mathrm{~mL}, 70 \mathrm{mmol}, 10$ equiv) was added to the reaction and the mixture was allowed to slowly warm to rt overnight. The reaction was then concentrated to afford the intermediate aldehyde as a yellow oil, which was carried on without further purification.

The crude aldehyde was dissolved in $\mathrm{MeOH}(35 \mathrm{~mL}, 0.2 \mathrm{M})$ and $\mathrm{BnNH}_{2}(2.3 \mathrm{~mL}, 21 \mathrm{mmol}$, 3 equiv) was added. The mixture was stirred at $\mathrm{rt}$ for $20 \mathrm{~min}$. Then $\mathrm{NaBH}_{3} \mathrm{CN}(0.342 \mathrm{~g}, 5.44 \mathrm{mmol}$, 0.78 equiv) was added in one portion and the mixture was stirred at $\mathrm{rt}$ for $1 \mathrm{hr}$. Another portion of $\mathrm{NaBH}_{3} \mathrm{CN}$ (0.34 g, 5.4 mmol, 0.78 equiv) was added and the mixture was stirred for 45 min. A final portion of $\mathrm{NaBH}_{3} \mathrm{CN}$ ( $1.5 \mathrm{~g}, 24 \mathrm{mmol}, 3.5$ equiv, $\sim 5$ equiv overall) was added followed by $\mathrm{AcOH}$ (1.2 mL, $21 \mathrm{mmol}, 3$ equiv). The reaction was then stirred at rt overnight $(\sim 16 \mathrm{hrs})$. The reaction was then concentrated to a viscous yellow oil. The residue was taken up in $\mathrm{CH}_{2} \mathrm{Cl}_{2}$ (30 $\mathrm{mL}$ ) and washed with sat. aq. $\mathrm{NaHCO}_{3}\left(30 \mathrm{~mL}\right.$ x 2). The organic layer was then dried over $\mathrm{MgSO}_{4}$, filtered, and concentrated to an orange oil. The residue was purified by column chromatography $\left(\mathrm{SiO}_{2}\right.$ neutralized with 5\% $\mathrm{Et}_{3} \mathrm{~N}$ in Hexanes, 1:0 $\rightarrow 4: 1 \mathrm{Hex} / \mathrm{EtOAc}$ ) to afford the transazabicyclononane S29 (190 $\mathrm{mg}, 0.697 \mathrm{mmol}, 10 \%)$ as a light orange solid and cisazabicyclononane $\mathbf{S 3 0}$ (761.9 $\mathrm{mg}, 2.78 \mathrm{mmol}, 40 \%)$ as a pale yellow oil. $\mathrm{R}_{\mathrm{f}}=0.32$ and $0.15(4: 1$ $\mathrm{Hex} /$ EtOAc, visualized with $\mathrm{KMnO}_{4}$ stain), respectively.

\section{Trans-azabicyclononane $\mathbf{S 2 9}$}

${ }^{1}$ H NMR $\left(600 \mathrm{MHz}, \mathrm{CDCl}_{3}\right) \delta$ 7.38-7.35 (m, 1H), 7.34-7.30 (m, 4H), $4.27(\mathrm{q}, J=7.1 \mathrm{~Hz}, 2 \mathrm{H})$, $3.96(\mathrm{~d}, J=13.5 \mathrm{~Hz}, 1 \mathrm{H}), 3.53(\operatorname{app~t}, J=6.7 \mathrm{~Hz}, 2 \mathrm{H}), 2.91(\mathrm{dd}, J=7.2,9.2 \mathrm{~Hz}, 1 \mathrm{H}), 2.64(\mathrm{dd}, J$ $=6.4,12.5 \mathrm{~Hz}, 1 \mathrm{H}), 2.52(\mathrm{dt}, J=6.5,10.0 \mathrm{~Hz}, 1 \mathrm{H}), 2.42-2.36(\mathrm{~m}, 1 \mathrm{H}), 2.19-2.15(\mathrm{~m}, 1 \mathrm{H}), 2.09-$ $1.95(\mathrm{~m}, 2 \mathrm{H}), 1.94-1.88(\mathrm{~m}, 1 \mathrm{H}), 1.83-1.77(\mathrm{~m}, 1 \mathrm{H}), 1.63(\mathrm{ddd}, J=7.3,10.5,12.5 \mathrm{~Hz}, 1 \mathrm{H}), 1.35$ (t, $J=7.1 \mathrm{~Hz}, 3 \mathrm{H})$

${ }^{13} \mathrm{C}$ NMR $\left(150 \mathrm{MHz}, \mathrm{CDCl}_{3}\right) \delta 173.8,138.4,128.9,128.4,128.4,128.2,127.3,74.4,65.3,61.9$, $54.7,51.9,38.7,37.4,34.6,24.4,14.0$

IR (thin film, $\mathrm{cm}^{-1}$ ) 2938, 1729, 1452, 1288, 1159, 1025

HRMS Several attempts to acquire HRMS data for this product using ESI-TOF and CI-TOF ionization techniques were unsuccessful.

\section{Cis-azabicyclononane S30}

${ }^{1} \mathbf{H}$ NMR $\left(600 \mathrm{MHz}, \mathrm{CDCl}_{3}\right) \delta$ 7.34-7.28 (m, 4H), 7.25-7.21 (m, 1H), $4.14(\mathrm{q}, J=7.1 \mathrm{~Hz}, 2 \mathrm{H})$, $3.87(\mathrm{~d}, J=13.1 \mathrm{~Hz}, 1 \mathrm{H}), 3.39(\mathrm{~d}, J=13.1 \mathrm{~Hz}, 1 \mathrm{H}), 3.19(\mathrm{~d}, J=5.8 \mathrm{~Hz}, 1 \mathrm{H}), 2.82(\operatorname{app~t}, J=7.7$ $\mathrm{Hz}, 1 \mathrm{H}), 2.37(\mathrm{dd}, J=5.6,12.3 \mathrm{~Hz}, 1 \mathrm{H}), 2.28(\mathrm{ddd}, J=5.6,9.0,11.1 \mathrm{~Hz}, 1 \mathrm{H}), 1.99-1.92(\mathrm{~m}, 1 \mathrm{H})$, $1.88-1.78(\mathrm{~m}, 1 \mathrm{H}), 1.72-1.58(\mathrm{~m}, 4 \mathrm{H}), 1.54-1.46(\mathrm{~m}, 1 \mathrm{H}), 1.26(\mathrm{t}, J=7.1 \mathrm{~Hz}, 3 \mathrm{H})$ 
${ }^{13} \mathrm{C}$ NMR $\left(150 \mathrm{MHz}, \mathrm{CDCl}_{3}\right) \delta 177.7,139.8,128.8,128.1,126.8,74.1,60.6,59.4,59.1,53.8$, $38.0,36.3,32.6,25.0,14.2$

IR (thin film, $\mathrm{cm}^{-1}$ ) 2951, 2865, 1725, 1452, 1160

HRMS Several attempts to acquire HRMS data for this product using ESI-TOF and CI-TOF ionization techniques were unsuccessful.

NHPI Ester S31: A $100 \mathrm{~mL}$ round-bottom flask was charged with $\mathbf{S 3 0}^{33}(1.64 \mathrm{~g}, 6.00 \mathrm{mmol})$ and 1,2-dichloroethane $(60 \mathrm{~mL}, 0.1 \mathrm{M})$. Methyl chloroformate $(2.8 \mathrm{~mL}, 36 \mathrm{mmol}, 6$ equiv) was added and the mixture was heated to reflux for $24 \mathrm{~h}$. The reaction was then concentrated to a brown oil and then flushed through a $\mathrm{SiO}_{2}$ plug with 4:1 $\mathrm{Hex} / \operatorname{EtOAc}(500 \mathrm{~mL})$. The filtrate was concentrated to a yellow oil $(1.12 \mathrm{~g})$, which was carried on without further purification.

The crude ester was then dissolved in a $2: 1$ mixture of $\mathrm{MeOH}: \mathrm{H}_{2} \mathrm{O}(9 \mathrm{~mL}, 0.5 \mathrm{M})$ and $\mathrm{LiOH} \cdot \mathrm{H}_{2} \mathrm{O}(293.6 \mathrm{mg}, 7.00 \mathrm{mmol}, 1.5$ equiv) was added. The flask was sealed and the mixture was heated to $60{ }^{\circ} \mathrm{C}$ for $18 \mathrm{hr}$. The reaction was then cooled to $\mathrm{rt}$ and the $\mathrm{MeOH}$ was removed under vacuum. The mixture was then acidified with $1 \mathrm{M}$ aq. $\mathrm{HCl}(10 \mathrm{~mL})$ and the mixture was extracted with EtOAc $(20 \mathrm{~mL}$ x 3$)$. The combined organic layers were then dried over $\mathrm{MgSO}_{4}$, filtered, and concentrated to an orange oil that solidified under vacuum $(931 \mathrm{mg})$. The crude acid was carried on without further purification.

The crude acid was dissolved in $\mathrm{CH}_{2} \mathrm{Cl}_{2}(22 \mathrm{~mL}, 0.2 \mathrm{M})$ along with $\mathrm{EDC} \cdot \mathrm{HCl}(1.26 \mathrm{~g}, 6.56$ mmol, 1.5 equiv) and $\mathrm{Et}_{3} \mathrm{~N}(1.2 \mathrm{~mL}, 8.7 \mathrm{mmol}, 2$ equiv). The mixture was stirred for $5 \mathrm{~min}$ at $\mathrm{rt}$, then N-hydroxyphthalimide ( $1.06 \mathrm{~g}, 6.56 \mathrm{mmol}, 1.5$ equiv) was added. The resulting red solution was stirred overnight at rt. The reaction was then quenched by the addition of $1 \mathrm{M}$ aq. $\mathrm{HCl}(20$ $\mathrm{mL}$ ) and the mixture was extracted with $\mathrm{CH}_{2} \mathrm{Cl}_{2}(20 \mathrm{~mL} \times 2)$. The combined organic layers were then washed with sat. aq. $\mathrm{NaHCO}_{3}(30 \mathrm{~mL})$ and dried over $\mathrm{MgSO}_{4}$, filtered, and concentrated to an orange foam. The crude residue was purified by flash column chromatography $\left(\mathrm{SiO}_{2}\right.$, $4: 1 \rightarrow 2: 1 \rightarrow 1: 1 \mathrm{Hex} / \mathrm{EtOAc}$ ) to afford NHPI ester $\mathbf{S 3 1}$ ( $878.6 \mathrm{mg}, 41 \%$ over 3 steps) as a colorless solid. $\mathrm{R}_{\mathrm{f}}=0.64(1: 1 \mathrm{Hex} / \mathrm{EtOAc})$.

(Note: NHP ester $\mathbf{S 3 1}$ exists as a mixture of rotamers leading to the broadening of ${ }^{1} \mathrm{H}$ NMR peaks and extra ${ }^{13} \mathrm{C}$ NMR peaks).

${ }^{1} \mathbf{H}$ NMR $\left(600 \mathrm{MHz}, \mathrm{CDCl}_{3}\right) \delta 7.89(\mathrm{dd}, J=3.1,4.7 \mathrm{~Hz}, 2 \mathrm{H}), 7.80(\mathrm{dd}, J=3.2,4.7 \mathrm{~Hz}, 2 \mathrm{H}), 4.75-$ $4.59(\mathrm{~m}, 1 \mathrm{H}), 3.87-3.62(\mathrm{~m}, 1 \mathrm{H}), 3.72(\mathrm{~s}, 3 \mathrm{H}), 3.50(\mathrm{q}, J=8.3 \mathrm{~Hz}, 1 \mathrm{H}), 2.65-2.53(\mathrm{~m}, 1 \mathrm{H}), 2.47-$ $2.37(\mathrm{~m}, 1 \mathrm{H}), 2.15-1.71(\mathrm{~m}, 6 \mathrm{H})$

${ }^{13}$ C NMR $\left(150 \mathrm{MHz}, \mathrm{CDCl}_{3}\right) \delta 172.2,162.0,155.0,134.9,129.0,124.1,66.9,66.7,66.3,52.6$, $52.4,46.7,46.4,37.0,35.1,34.6,34.5,33.8,29.7,25.2$

IR (thin film) 2957, 2918, 2874, 1808, 1782, 1740, 1697, 1448, 1383, 1275, 1260, 1185, 1125, 1080, 1046, 971

HRMS (ESI-TOF) m/z: [M+Na] calcd for $\mathrm{C}_{18} \mathrm{H}_{18} \mathrm{~N}_{2} \mathrm{O}_{6} \mathrm{Na} 381.1063$, found 381.1065 


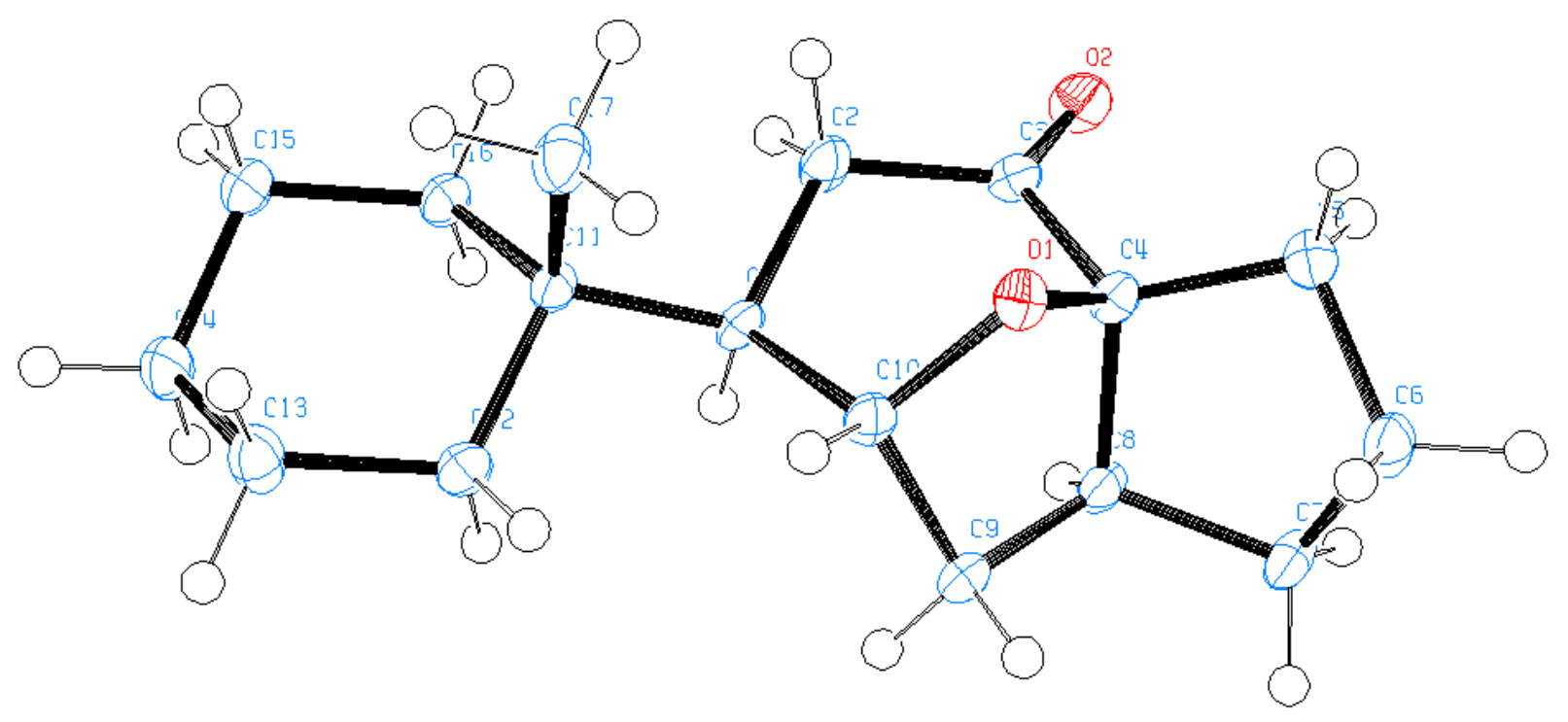

The thermal ellipsoid plot is shown at the $50 \%$ probability level and $\mathrm{H}$ atoms are shown as small spheres of arbitrary radii; Blue $=$ Carbon, Red $=$ Oxygen .

Table S10. Crystal data and structure refinement for leo299.

Identification code

Empirical formula

Formula weight

Temperature

Wavelength

Crystal system

Space group

Unit cell dimensions

Volume

Z

Density (calculated)

Absorption coefficient

$\mathrm{F}(000)$

Crystal color

Crystal size

Theta range for data collection

Index ranges

Reflections collected

Independent reflections

Completeness to theta $=25.242^{\circ}$ leo299 (Tyler Allred)

$\mathrm{C}_{17} \mathrm{H}_{26} \mathrm{O}_{2}$

262.38

133(2) K

$0.71073 \AA$

Orthorhombic

$P 2{ }_{1} 2_{1} 2_{1}$

$\mathrm{a}=7.6977(7) \AA \quad \alpha=90^{\circ}$.

$\mathrm{b}=8.6508(8) \AA \quad \beta=90^{\circ}$.

$\mathrm{c}=21.9047(19) \AA \quad \gamma=90^{\circ}$.

1458.7(2) $\AA^{3}$

4

$1.195 \mathrm{Mg} / \mathrm{m}^{3}$

$0.076 \mathrm{~mm}^{-1}$

576

colorless

$0.539 \times 0.292 \times 0.150 \mathrm{~mm}^{3}$

1.859 to $29.124^{\circ}$

$-10 \leq h \leq 10,-11 \leq k \leq 11,-30 \leq l \leq 30$

31185

$3924[\mathrm{R}(\mathrm{int})=0.0414]$

$100.0 \%$ 
Absorption correction

Max. and min. transmission

Refinement method

Data / restraints / parameters

Goodness-of-fit on $\mathrm{F}^{2}$

Final R indices $[\mathrm{I}>2 \operatorname{sigma}(\mathrm{I})=3692$ data $]$

$\mathrm{R}$ indices (all data, $0.73 \AA$ )

Largest diff. peak and hole
Semi-empirical from equivalents

0.8622 and 0.7258

Full-matrix least-squares on $\mathrm{F}^{2}$

3924 / 0 / 276

1.064

$\mathrm{R} 1=0.0376, \mathrm{wR} 2=0.0947$

$\mathrm{R} 1=0.0416, \mathrm{wR} 2=0.0974$

0.358 and -0.183 e. $\AA^{-3}$ 


\section{Q. References}

1. Lackner, G. L.; Quasdorf, K. W.; Overman, L. E. J. Am. Chem. Soc. 2013, 135(41), 15342-15345.

2. Lo, J. C.; Yabe, Y.; Baran, P. S. J. Am. Chem. Soc. 2014, 136(4), 1304-1307.

3. Qin, T.; Malins, L.R.; Edwards, J.T.; Merchant, R.R.; Novak, A.J.E.; Zhong, J.Z.; Mills, R.B.; Yan, M.; Yuan, C.; Eastgate, M.D.; Baran, P.S. Angew. Chem. Int. Ed. 2017, 56(1), 260-265.

4. El-Hage, F,; Schöll, C.; Pospech, J. J. Org. Chem. 2020, 85(21), 13853-13867.

5. Dang, H. T.; Haug, G. C.; Nguten, V. T.; Vuong, N. T. H.; Nguyen, V. D.; Arman, H. D.; Larionov, O. V. ACS Catal. 2020, 10(19), 11448-11457.

6. Park, H.; Chekshin, N.; Shen, P.-X.; Yu, J.-Q. ACS Catal. 2018, 8(10), 9292-9297.

7. Chan, C.-O.; Crich, D.; Natarajan, S. Tetrahedron Lett. 1992, 33, 3405-3408.

8. Haut, F.-L.; Habiger, C.; Speck, K.; Wurst, K.; Mayer, P.; Korber, J.N.; Müller, T.; Magauer, T. J. Am. Chem. Soc. 2019, 141(34), 13352-13357.

9. Nicolaou, K. C.; Montagnon, T.; Baran, P.S.; Zhong, Y.-L. J. Am. Chem. Soc. 2002, 124(10), $2245-2258$.

10. Chong, B.-D.; Ji, Y.-I.; Oh, S.-S.; Yang, J.-D.; Baik, W.; Koo, S. J. Org. Chem. 1997, 62(26), 93239325.

11. Samame, R.A.; Owens, C.M.; Rychnovsky, S.D. Chem. Sci. 2016, 7, 188-190.

12. Roosen, P.C. \& Vanderwal, C.D. Org. Lett. 2014, 16(17), 4368-4371.

13. Tang, F.; Banwell, M.G.; Willis, A.C. J. Org. Chem. 2016, 81(7), 2950-2957.

14. Chen, K., Ishihara, Y.; Galán, M.M.; Baran, P.S. Tetrahedron, 2010, 66(26), 4738-4744.

15. Diao, T. \& Stahl, S.S. J. Am. Chem. Soc. 2011, 133(37), 14566-14569.

16. Schuster, H.; Martinez, R.; Bruss, H.; Antonchick, A.P.; Kaiser, M.; Schürmannb, M.; Waldmann, H. Chem. Commun. 2011, 47(23), 6545-6547.

17. Barros, M.T.; Maycock, C.D.; Ventura, M.R. J. Chem. Soc., Perkins Trans 1, 2001, 2001(2), $166-173$.

18. Erica L. Woodall, E.L.; Simanis, J.A.; Hamaker, C.G.; Goodell, J.R.; Mitchell, T.A. Org. Lett. 2013, 15(13), 3270-3273.

19. Slutskyy, Y.; Jamison, C.R.; Lackner, G.L.; Muller, D.S.; Dieskau, A.P.; Unteidt, N.L.; Overman, L.E. J. Org. Chem. 2016, 81, 7029-7035.

20. Cren, S.; Schär, P.; Renaud, P.; Schenk, K. J. Org. Chem. 2009, 74(8), 2942-2946.

21. Pratsch, G.; Lackner, G.L.; Overman, L.E. J. Org. Chem. 2015, 80(12), 6025-6036.

22. Wang, C.; Dong, G. J. Am. Chem. Soc. 2018, 140(19), 6057-6061.

23. Baran, P.; Li, C.; Wang, J.; Chatterjee, A.K.; Kumar, M.; Yu, S.; Johnson, K.A.; Qin, T.; Shang, M. Cuand Ni-catalyzed decarboxylative borylation reactions. U.S. Patent WO 2018175173, March 14, 2018.

24. Qin, T.; Cornella, J.; Li, C.; Malins, L.R.; Edwards, J.T.; Kawamura, S.; Maxwell, B.D.; Eastgate, M.D.; Baran, P.S. Science, 2016, 352, 801-805.

25. The stereochemistry of ketone $\mathbf{3 6}$ was assigned based on the ${ }^{1} \mathrm{H}$ NMR coupling constants observed for the hydrogen at $\mathrm{C} 4(4.09, \mathrm{dd}, J=9.3,10.7 \mathrm{~Hz})$.

26. Benesi, H.A.; Hildebrand, J.H. J. Am. Chem. Soc. 1949, 71(8), 2703-2707.

27. Richter, J.M.; Ishihara, Y.; Masuda, T.; Whitefield, B.W.; Llamas, T.; Pohjakallio, A.; Baran, P.S. J. Am. Chem. Soc. 2008, 130(52), 17938-17954.

28. Leonidas-Dimitrios Syntrivanis, L.-D.; Némethová, I.; Schmid, D.; Levi, S.; Prescimone, A.; Bissegger, F.; Major, D.T.; Tiefenbacher, K. J. Am. Chem. Soc. 2020, 142(12), 5894-5900.

29. Jouanneau, M.; Vellalath, S.; Kang, G.; Romo, D. Tetrahedron, 2019, 75(24), 3348-3354.

30. Weires, N.A.; Slutskyy, Y.; Overman, L.E. Angew. Chem. Int. Ed. 2019, 58(25), 8561-8565.

31. Kim, H.-J.; Ricardo, A.; Illangkoon, H.I.; Kim, M.J.; Carrigan, M.A.; Frye, F.; Benner, S.A. J. Am. Chem. Soc. 2011, 133(24), 9457-9468.

32. Zi-Long Li, Z.-L.; Lv, A.; Du, F.-S.; Li, Z.-C. Macromolecules, 2014, 47(17), 5942-5951.

33. The trans-bicyclic amine does not react under these conditions. 


\section{R. NMR Spectra}




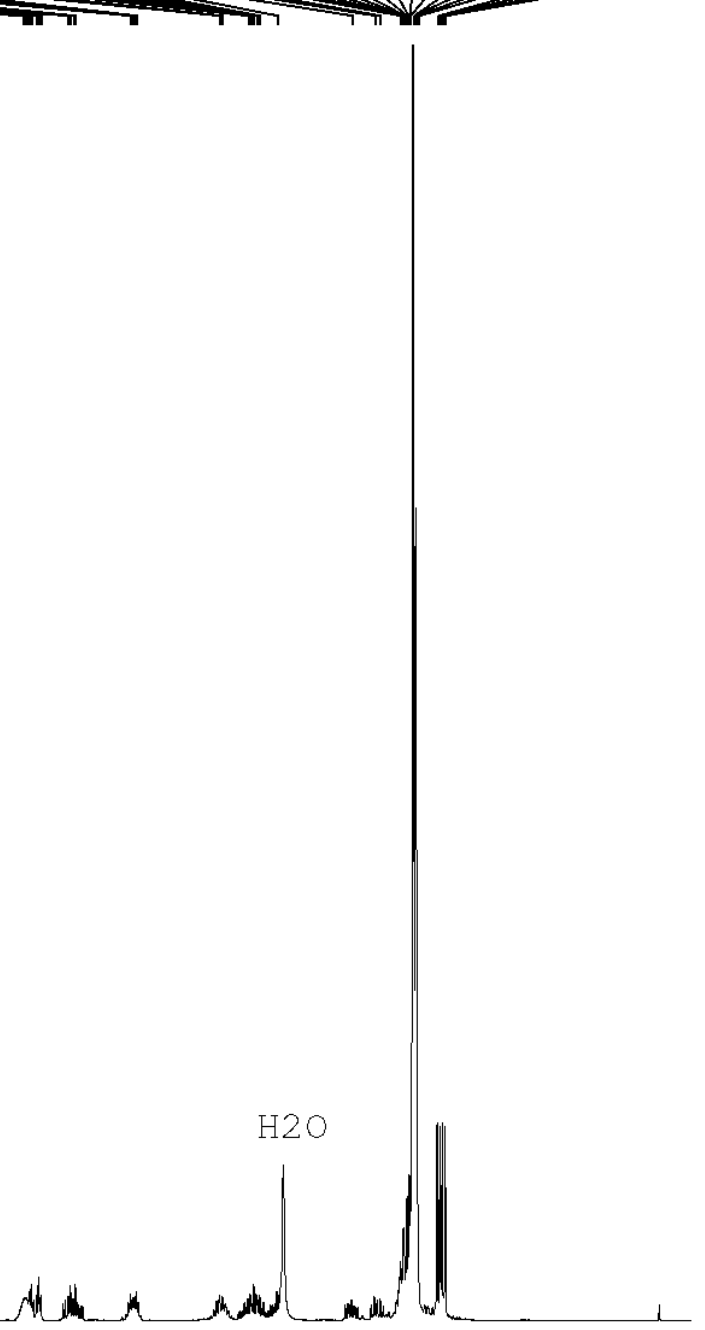

F2 - Acquisition Parame<smiles>CC(CO[18O])CC1C=CC(=O)CC1</smiles>

TD
SOLVENT

NS

$\begin{array}{lr}\text { DS } & 2 \\ \text { SWH } & 9615.385 \\ \text { FIDRES } & 0.098042\end{array}$

$\begin{array}{rr}\mathrm{AQ} & 0.098042 \\ \mathrm{BQ} & 5.0998478\end{array}$

RG

$\mathrm{DW}$

TE

D1
TDO

S1

\begin{tabular}{|c|c|c|}
\hline $\begin{array}{l}\text { SFO1 } \\
\text { NUC1 } \\
\text { P1 } \\
\text { PLW1 }\end{array}$ & & $\begin{array}{r}600.1342009 \\
1 \mathrm{H} \\
9.50 \\
30.00000000\end{array}$ \\
\hline F2 - & Proces & ssing paramet \\
\hline SI & & 65536 \\
\hline $\mathrm{SF}$ & & 600.1300356 \\
\hline WDW & & $\mathrm{EM}$ \\
\hline$S S B$ & 0 & \\
\hline $\mathrm{LB}$ & & 0.30 \\
\hline GB & 0 & \\
\hline PC & & 1.00 \\
\hline
\end{tabular}

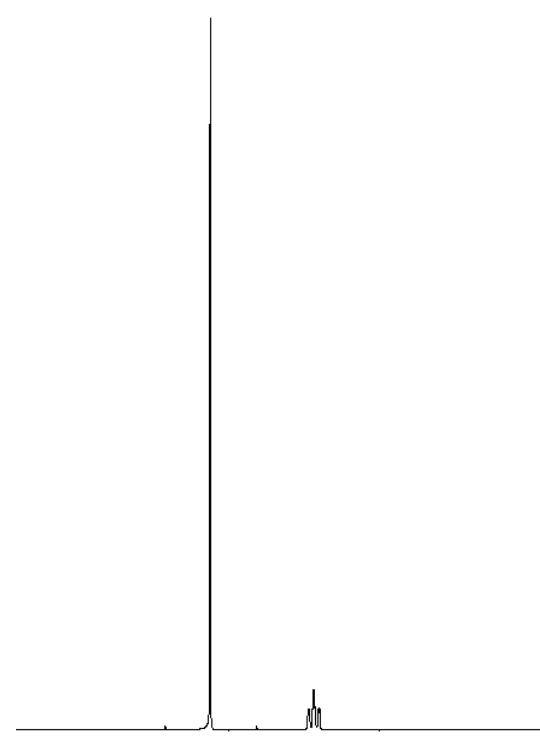

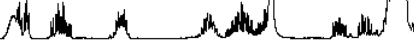

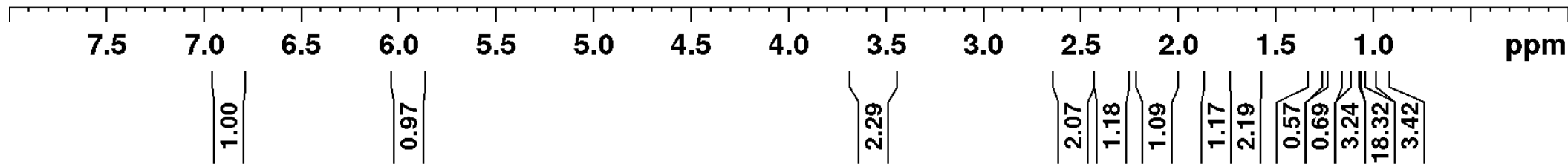




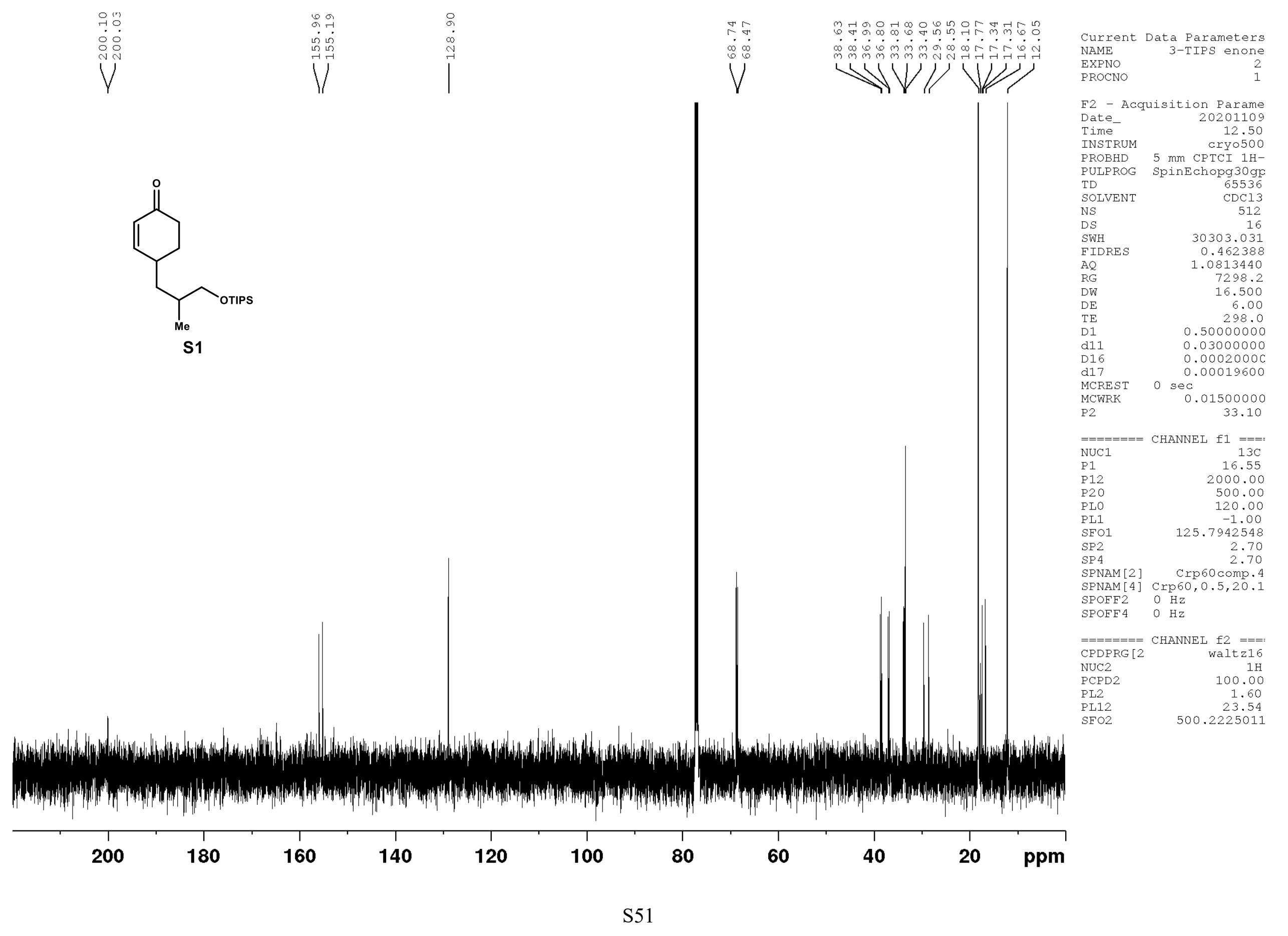




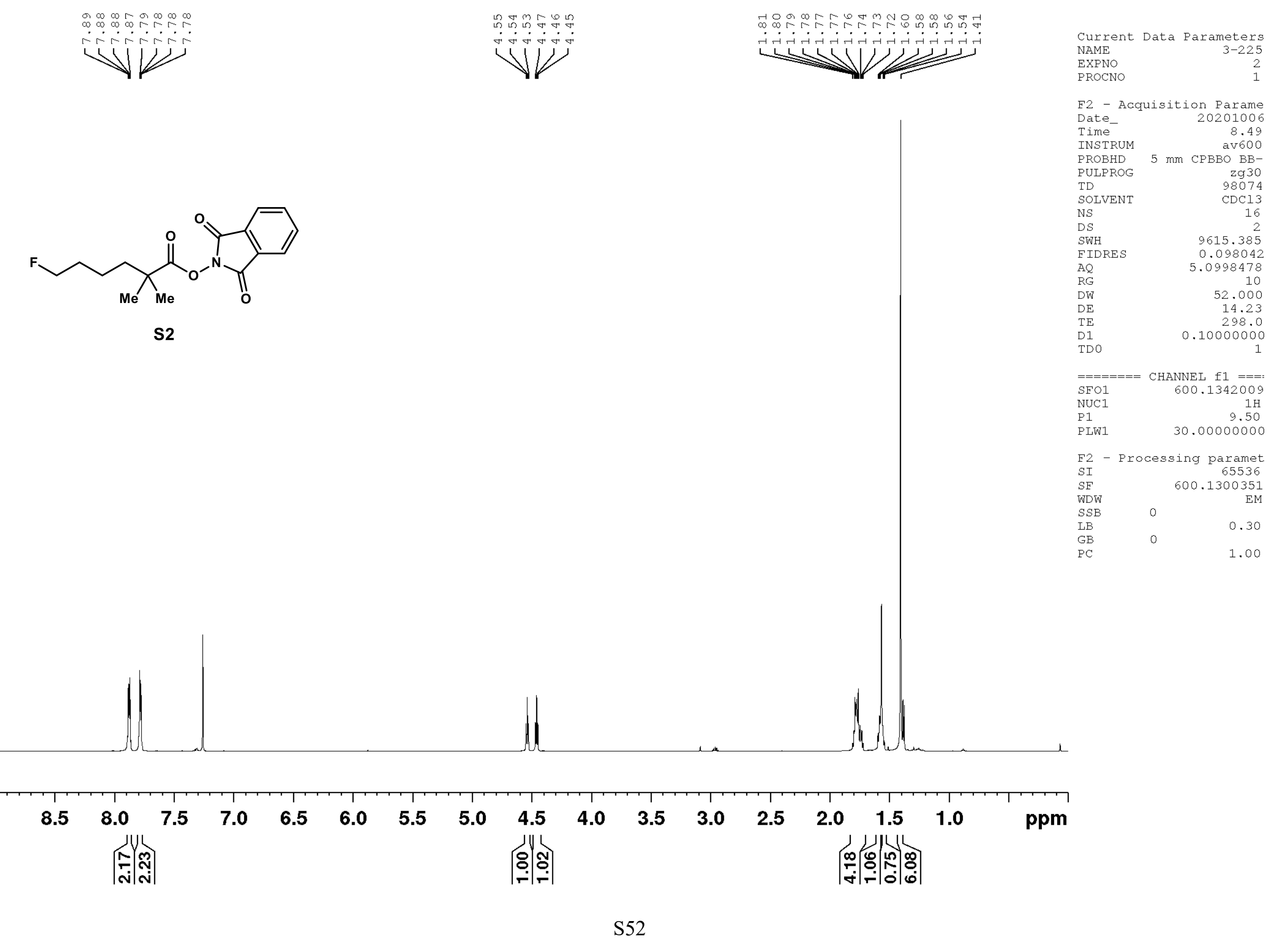



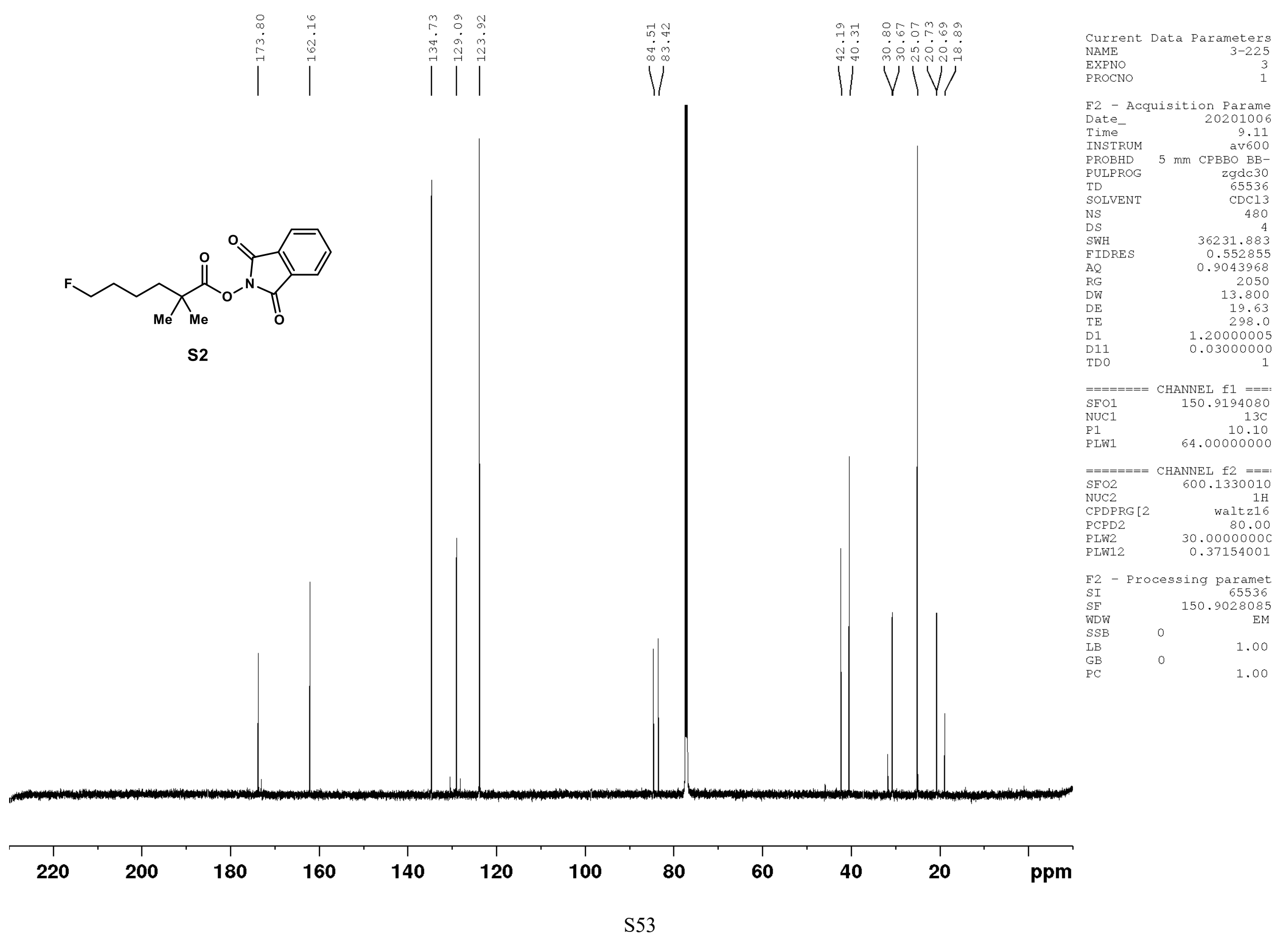

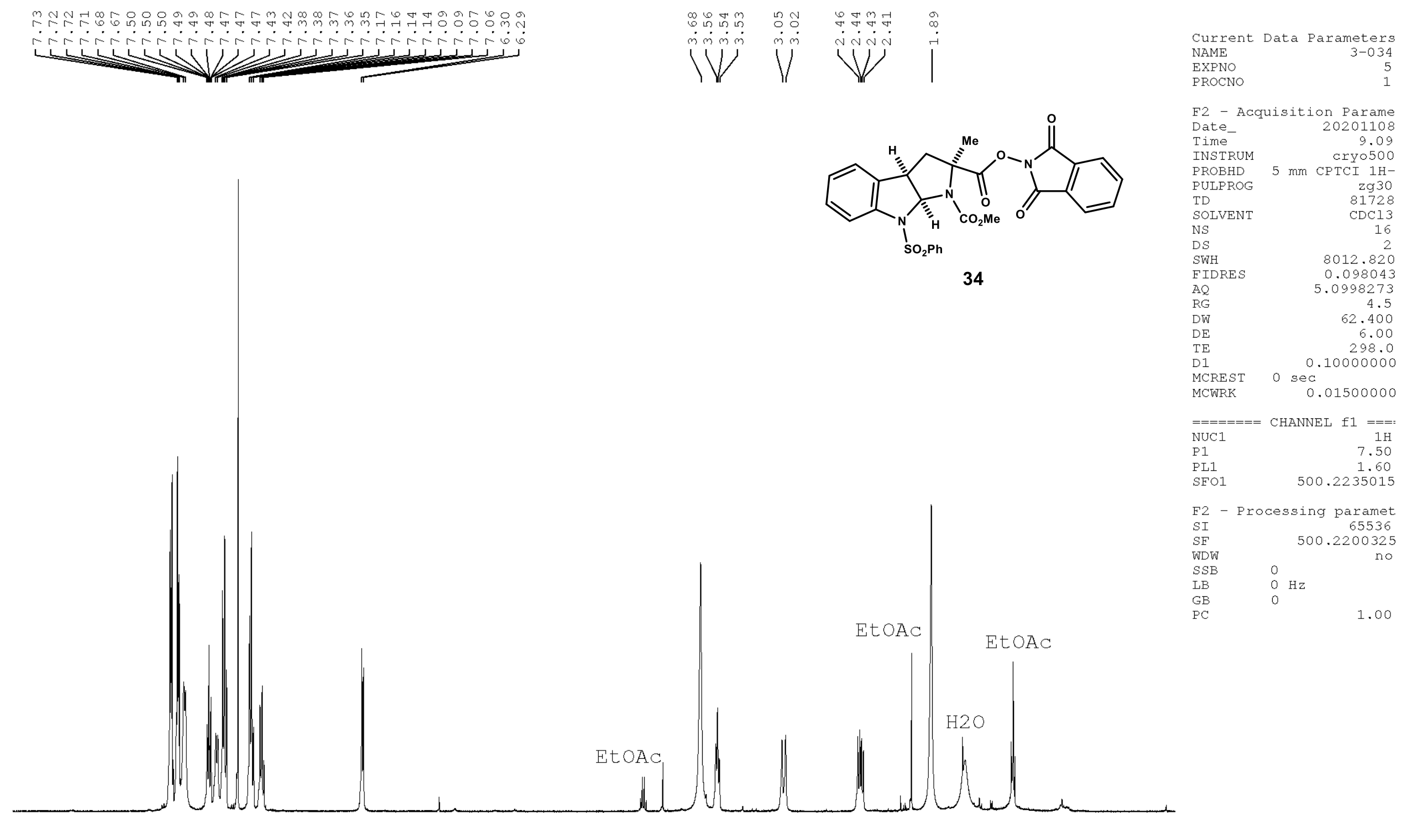

$\begin{array}{lllllllllllllllllll}8.5 & 8.0 & 7.5 & 7.0 & 6.5 & 6.0 & 5.5 & 5.0 & 4.5 & 4.0 & 3.5 & 3.0 & 2.5 & 2.0 & 1.5 & 1.0 & \mathrm{ppm}\end{array}$

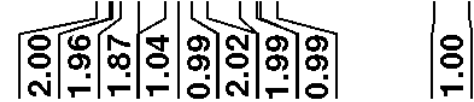

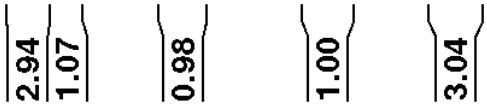



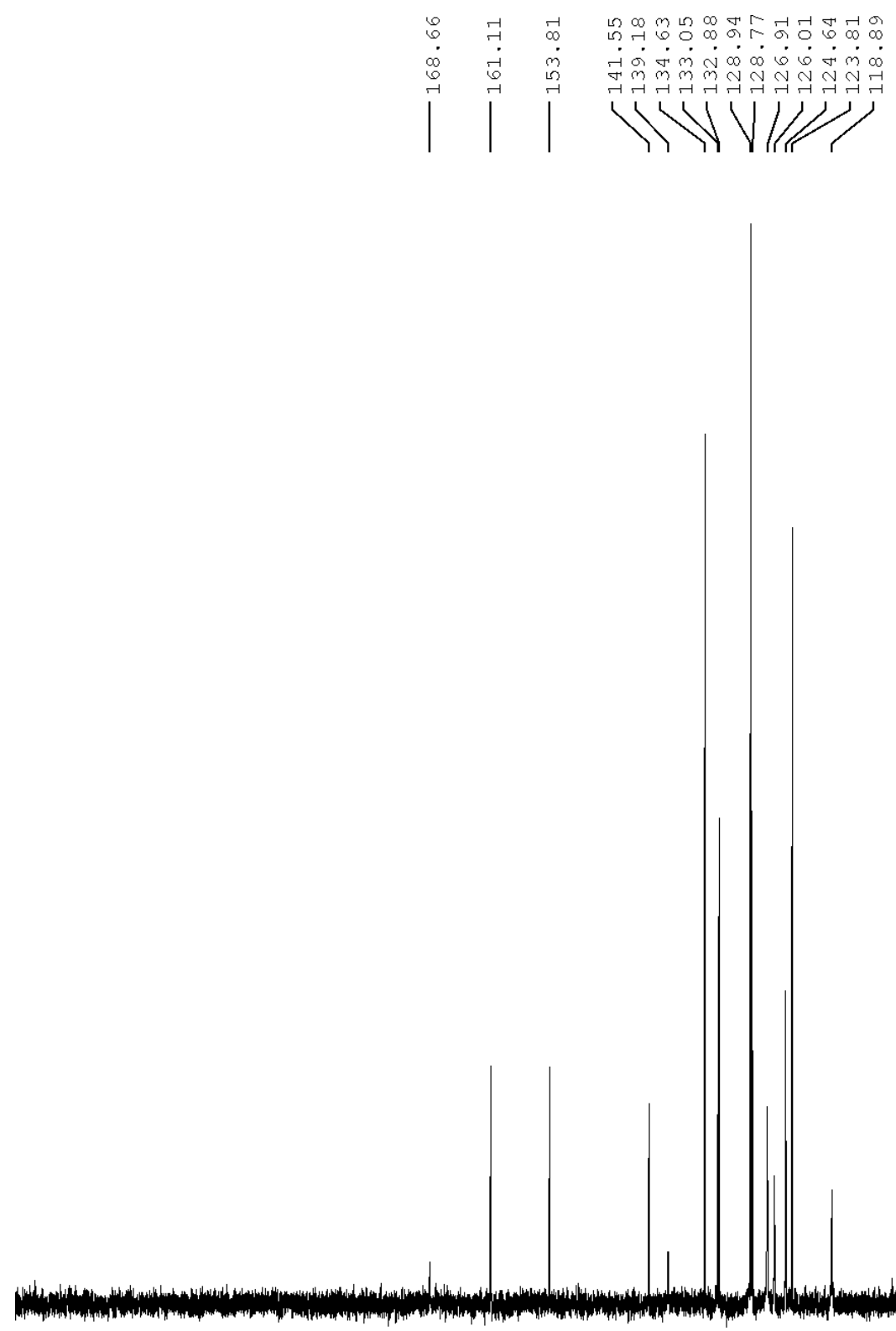

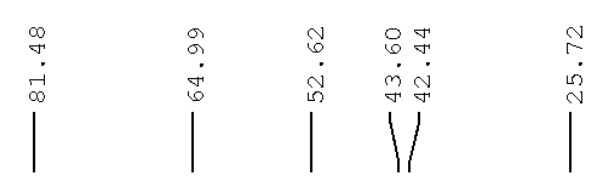

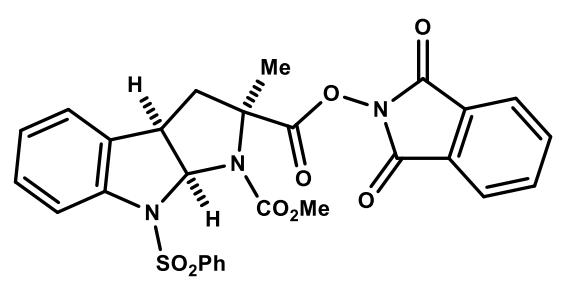

34
Curre

EXPNO

PROCNO

F2 - Acquisition Parame Date__ 20201108 Time

aryo500 PROBHD $5 \mathrm{~mm}$ CPTCI $1 \mathrm{H}-$ PULPROG SpinEchopg 30 gk TD

NS

NS

DS
SWH
FID

$\begin{array}{lr} & 16 \\ \text { WWH } & 30303.031\end{array}$

FIDRES $\quad 0.462388$ AQ $\quad 1.0813440$ $\begin{array}{lr}\text { RG } & 7298.2 \\ \text { DW } & 16.500\end{array}$ $\begin{array}{lr}\text { DW } & 16.500 \\ \text { DE } & 6.00\end{array}$ D1 $\quad 1.20000005$ d11 $\quad 0.03000000$ D16 $\quad 0.0002000 \mathrm{C}$ d17

$\begin{array}{ll}\text { MCREST } & 0 \text { sec } \\ \text { MCWRK } & 0.0150000 \mathrm{C}\end{array}$ P2

.01500000
33.10 $=======$ CHANNEL $\mathrm{f} 1===$. NUC1 $13 \mathrm{C}$ $\begin{array}{lr}\text { P1 } & 16.55\end{array}$ $\begin{array}{lr}\mathrm{P} 12 & 2000.00 \\ \mathrm{P} 20 & 500.00\end{array}$ $\begin{array}{ll}\text { P20 } & 500.00 \\ \text { PLO } & 120.00\end{array}$ PL1 -1.00 $\begin{array}{lr}\text { SP2 } & 125.7942548 \\ \text { SP2 } & 2.70\end{array}$ Crp60comp.4 SPOFF 2 O Hz

$=======$ CHANNEL $\mathrm{f} 2===$ CPDPRG

PCPD 2

$\mathrm{PL} 2$

$\mathrm{PL} 12$
$\mathrm{SFO} 2$ 


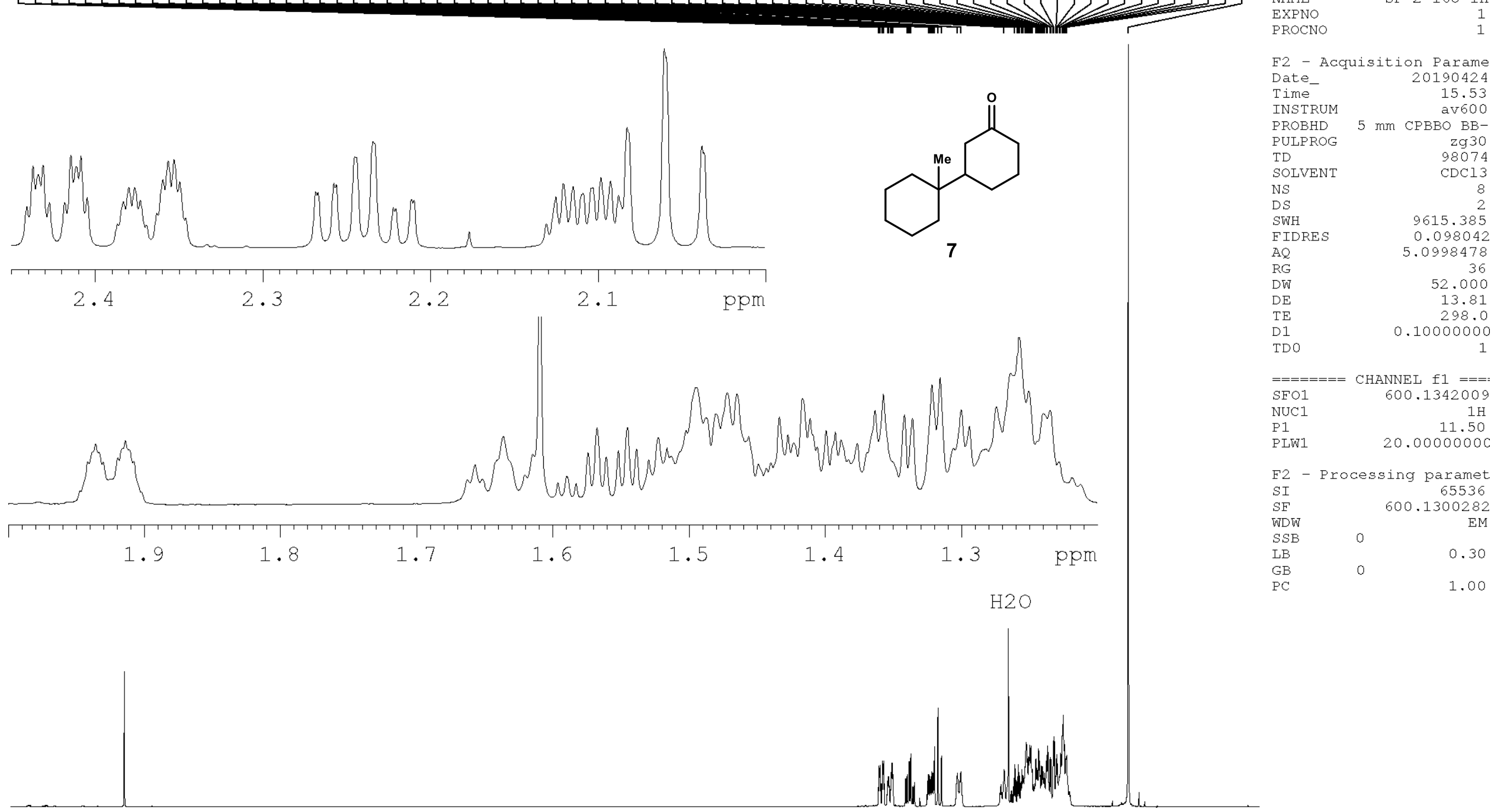

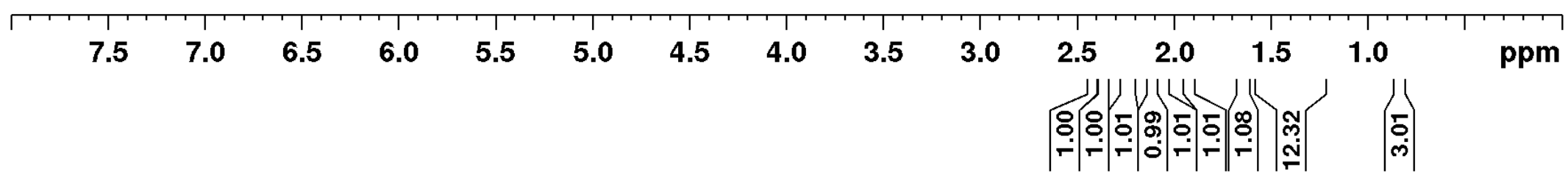


F2 - Acquisition Parame Date

Date

Time

PROBHD

TD

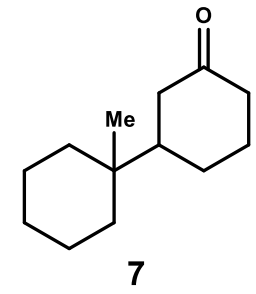

TD
SOLVENT

NS

DS

FIDRE

AQ

RG

DE

TE

D1

D11
TDO

$====$

SFO1
NUC1

P1

PLW1

20190424
15.46 15.46

$5 \mathrm{~mm}$ CPBBO BB-

65536

261
4

36231.883

0.552855 0.9043968 2050
13.800

18.01

0.40000001

0.03000000

CHANTET 11 =

150.9194080

$13 \mathrm{C}$
9.60

$=======$ CHAnTEL f2

$\mathrm{SFO} 2$

ANNEL $12===$

$1 \mathrm{H}$

CPDPRG [2 waltz16

20.00000000

PLW12

20.00000000
0.32734001

F2 - Processing paramet

SI

SF $\quad 150.9028085$

WDW

$S S B$

EM

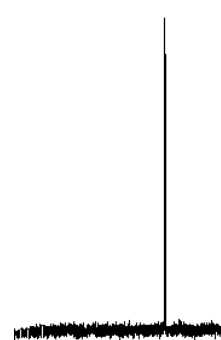

$L B$

GB

1.00

1.00

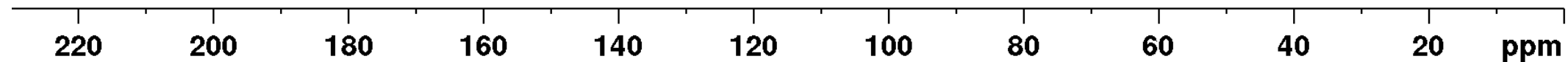


F2 - Acquisition Parame
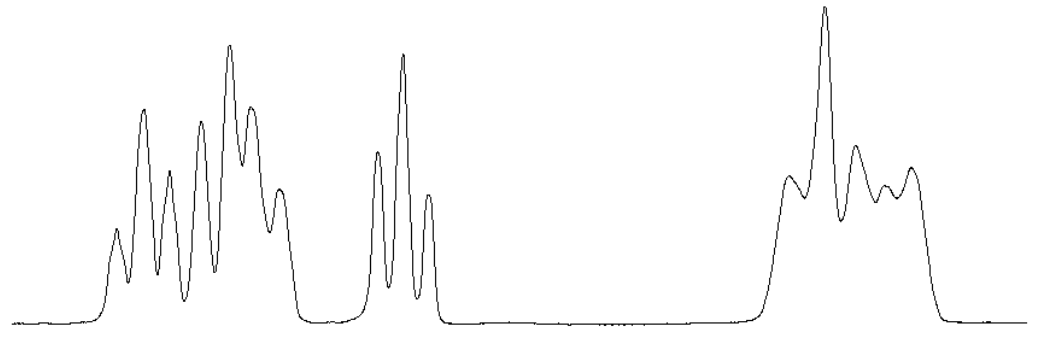

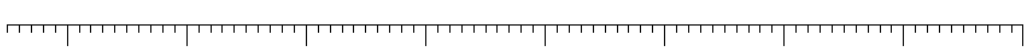
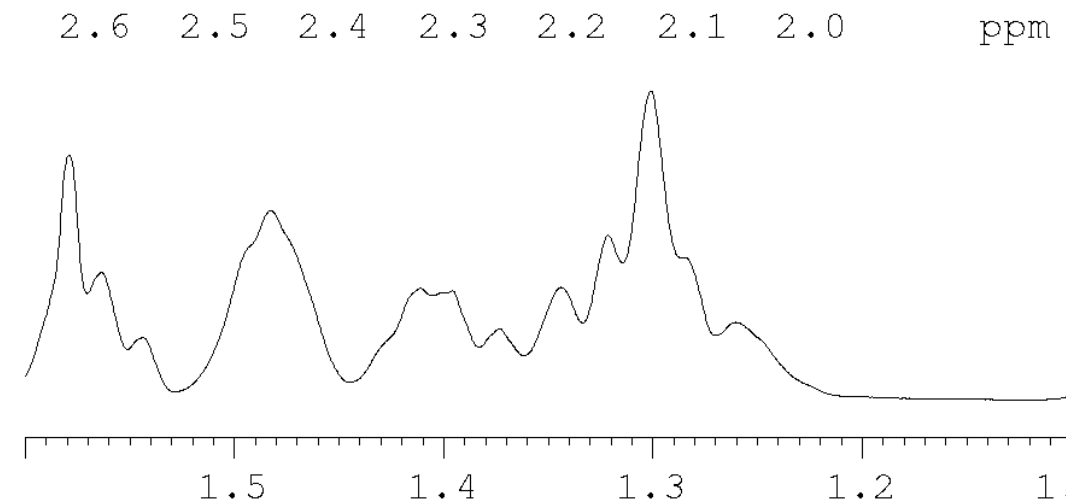

1.5

1.4

1.3

1.2

1.1

ppm

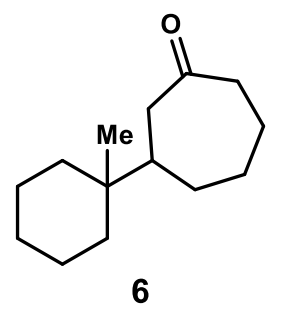

6

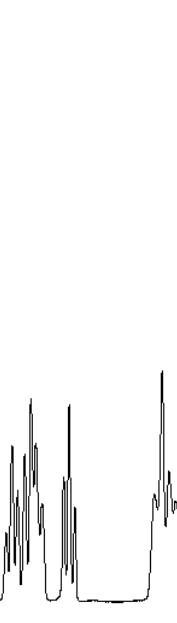

Date

Iime

INSTRUM

PROBHD
PULPROG

TD

SOLVEN

DS

FIDRES

$\mathrm{AQ}$
$\mathrm{RG}$

RG
DW

DW

TE
D1

D1
TDO

$===$

SFO1
NUC1

PUC1

PLW1

20190425
15.14

av 600

zg30
98074

CDCl3

8
2
9615.385

0.098042 5.0998478

52.000

13.81

0.10000000

CHANITET 600.1342009 $1 \mathrm{H}$
11.50

F2 - Processing paramet

$\begin{array}{lr}\text { SI } & 65536 \\ \text { SF } & 600.1300352\end{array}$

WDW

SSB

LB

GB

0.30

1.00

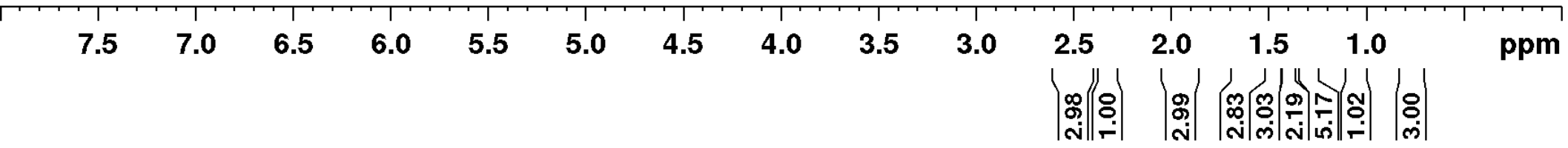




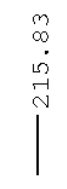

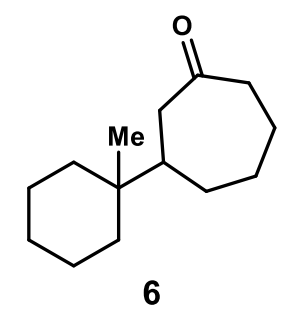

220

200

180

160

140

120

100

80

60

40

20

ppm 
F2 - Acquisition Parame
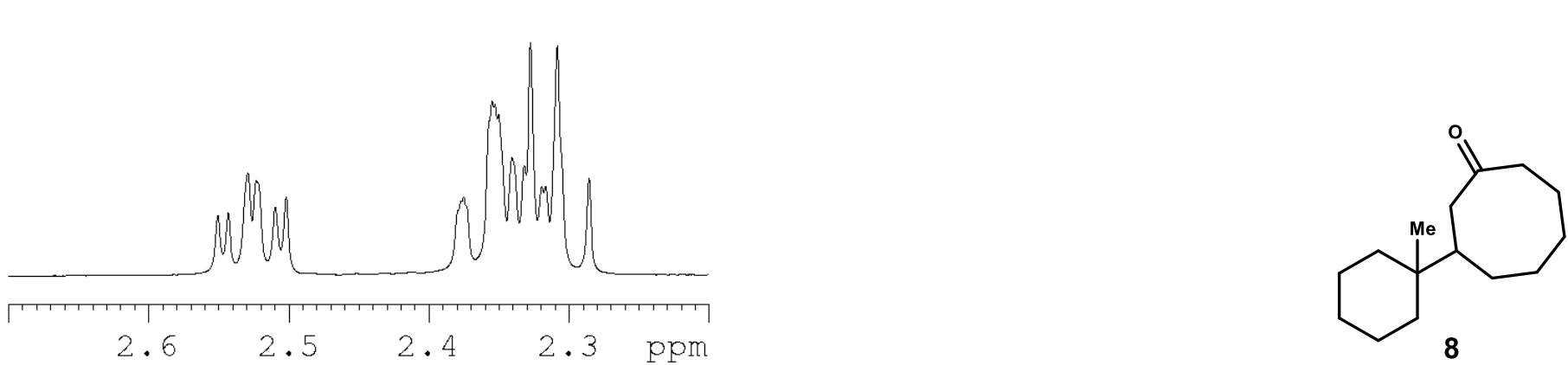

Time

av600

PROBHD $5 \mathrm{~mm}$ CPBBO BB-

$\begin{array}{lr}\text { PULPROG } & \text { zg30 } \\ \text { TD } & 98074\end{array}$

TD

NS

$\begin{array}{lr}\text { DS } & 2 \\ \text { SWH } & 9615.385 \\ \text { FTDRES } & 0.098042\end{array}$

$\begin{array}{ll}\mathrm{AQ} & 0.098042 \\ \mathrm{R} & 5.0998478\end{array}$

AQ
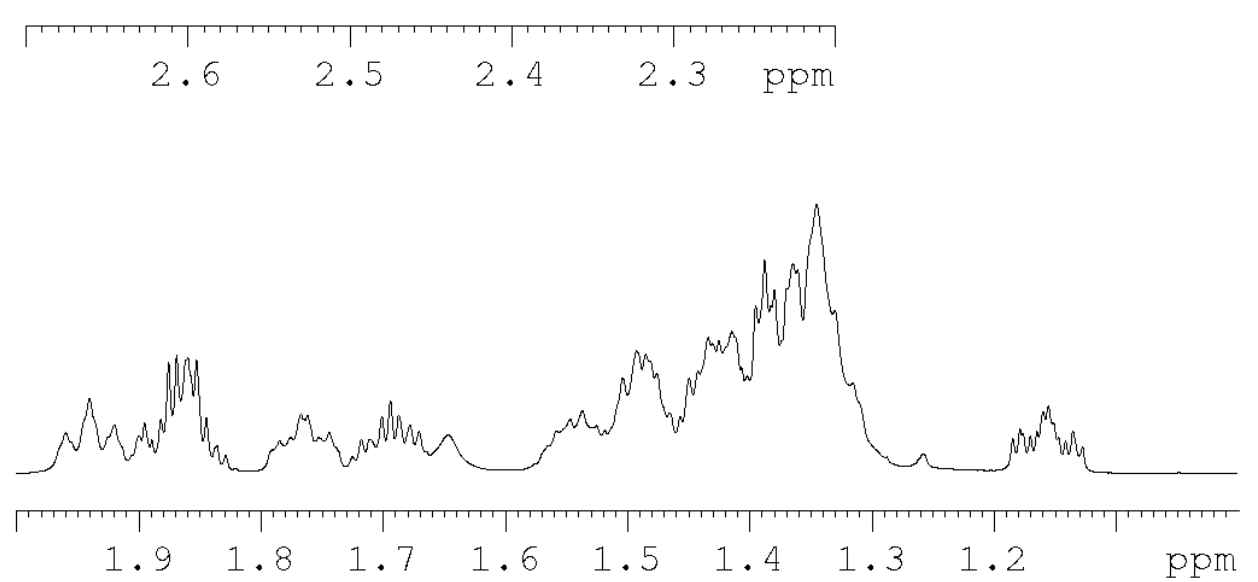

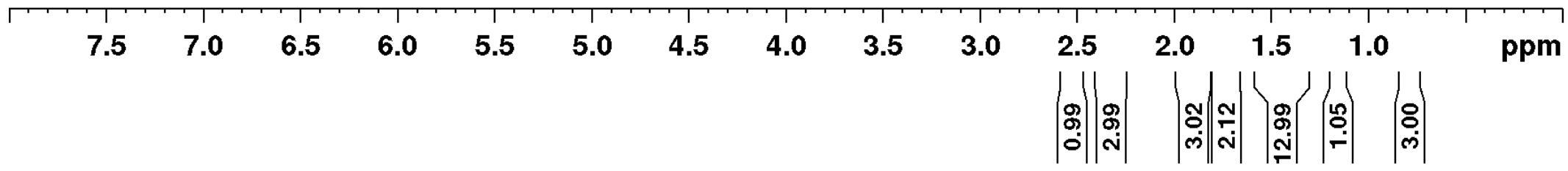




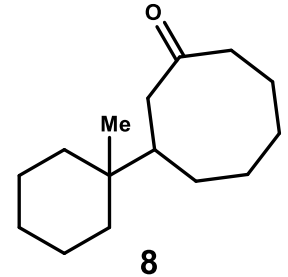

Date_

Time

PROBHD

PUI PROG

TD

SOLVENT

NS

DS

FIDRES

$\mathrm{AQ}$

RG
DW

$\mathrm{DE}$

TE

TE
D1
D 11

D11
TD0

$====$

SFO1
NUC1

P1
PLW1

\section{$==$}

$\mathrm{SFO} 2$

CPDPRG [2

PCPD2

PLW2
PLW12

F2 - Processing paramet

\section{CHANNEL $\mathrm{f} 1==$}

150.9194080
$13 \mathrm{C}$

10.10
64.0000000

64.00000000

CHANNEL f2 $===$

$1 \mathrm{H}$

30.00000000

.37154001

150.9028085

1.00

1.00

\begin{tabular}{|rrrrrrrrrrrr|r}
\hline 220 & 200 & 180 & 160 & 140 & 120 & 100 & 80 & 60 & 40 & 20 & ppm
\end{tabular}




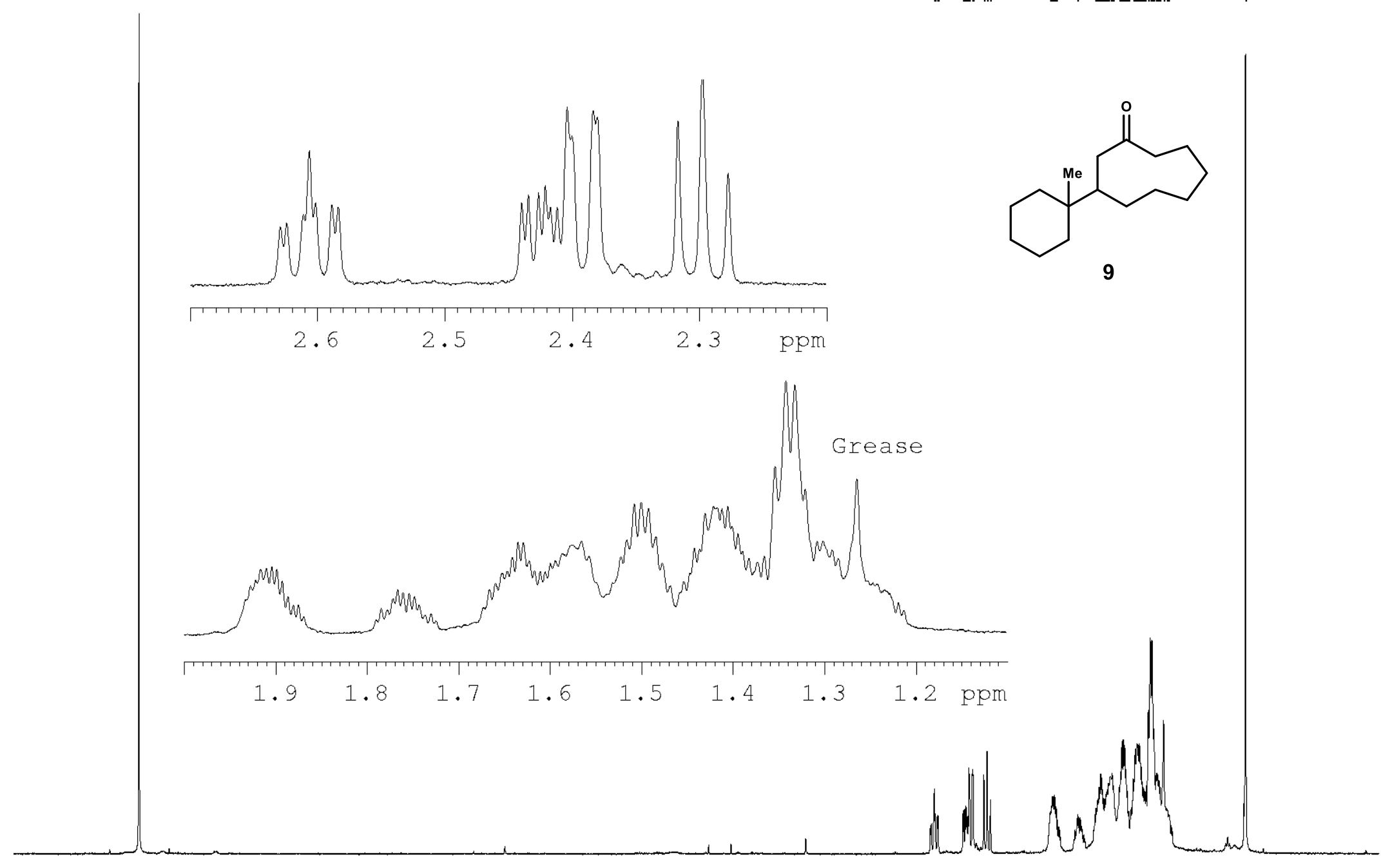

F2 - Acquisition Parame Date_ 20200303 $\begin{array}{lr}\text { Time } & 15.01 \\ \text { INSTRUM } & \text { av600 }\end{array}$ PROBHD $5 \mathrm{~mm}$ CPBBO BBPULPROG $\quad$ zg30 TD 98074 SOLVENT NS

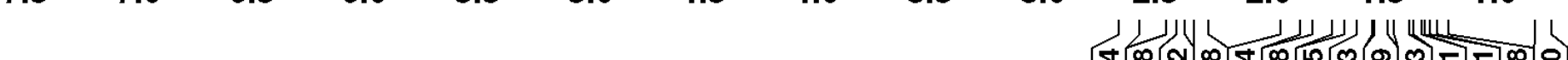 象}



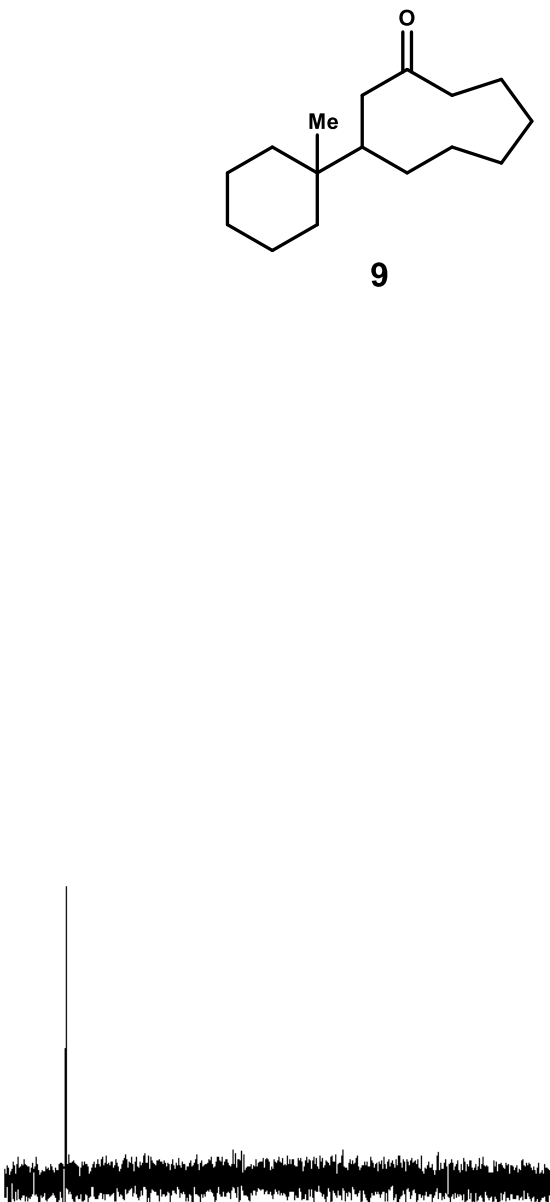

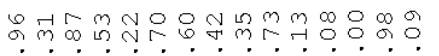

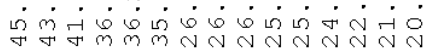
M Nill

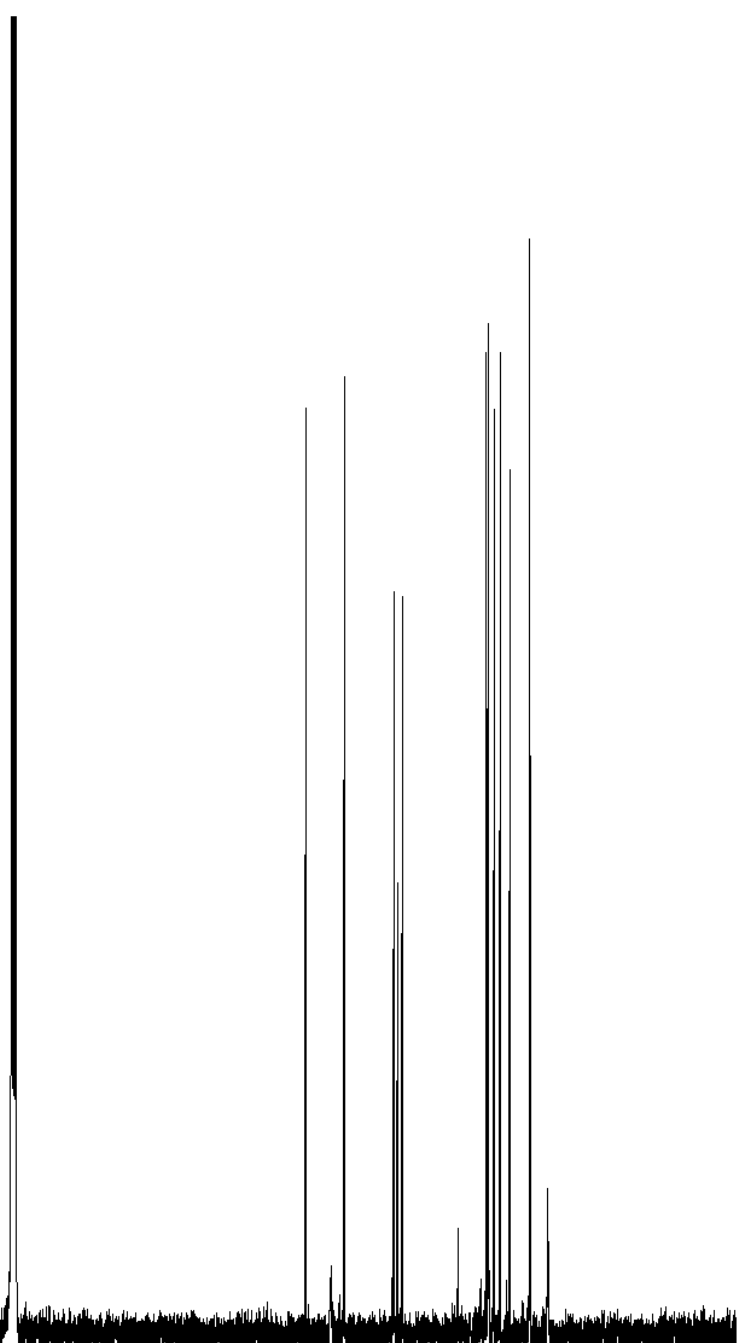

Grease
Current Data Parameters NAME

ExPNO

F2 - Acquisition Parame Date

INISTRUM 12.11
av600 $5 \mathrm{~mm} \mathrm{CPBBO} \mathrm{BB}-$ PULPROG 5 mn CPBBO TD 65536 SOLVENT NS

DS AQ $\quad 0.9043968$

$\mathrm{DE}$

TE

298.0 0.03000000
$D 11$$$
====
$$$$
\text { SFO1 }
$$$$
\text { NUC1 }
$$$$
\begin{aligned}
& \text { P1 } \\
& \text { PLW1 }
\end{aligned}
$$

CHANNEL $\mathrm{f} 1==$ 150.9194080
$13 \mathrm{C}$ 64.00000000

$========$ CHANNEL $\mathrm{f} 2===$ :

$\mathrm{SFO} 2600.1330010$

NUC2 $1 \mathrm{H}$

PCPD2 waltz16

PLW2 30.00000000

PLW12 0.37154001

F2 - Processing paramet
SI

SF $\quad 150.9028085$

WDW

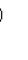

1.00

$\mathrm{PC}$

1.00

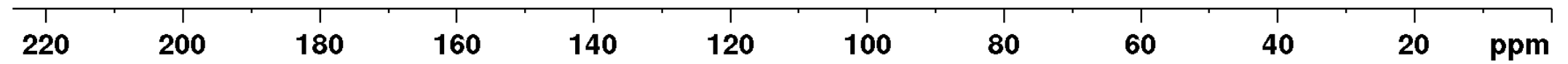



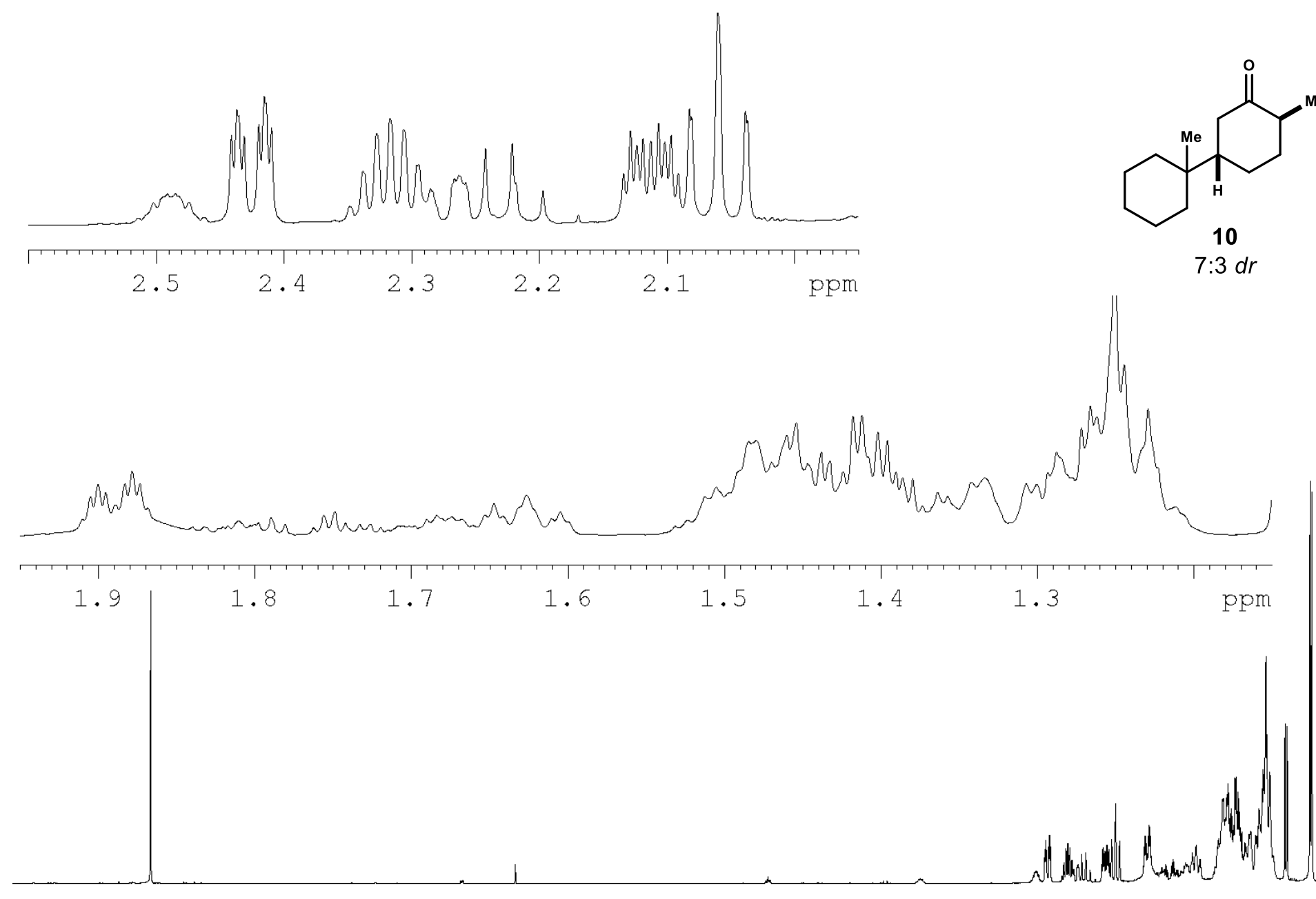

m
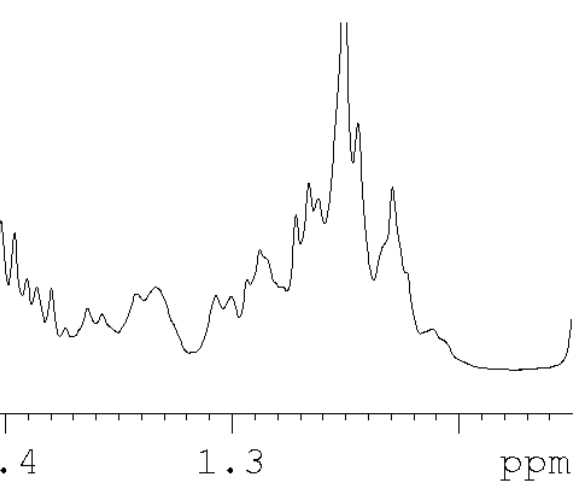

1.4

1.3

1.7

1.6

1.5$$
1.4
$$

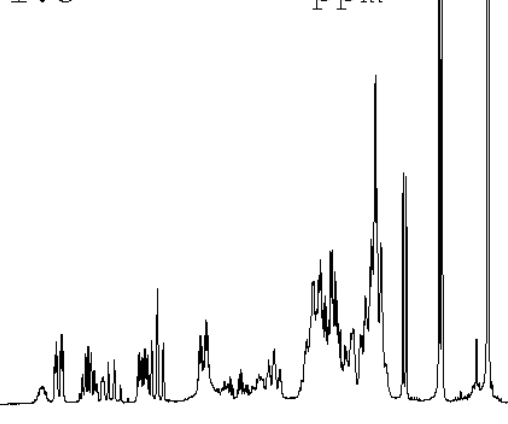

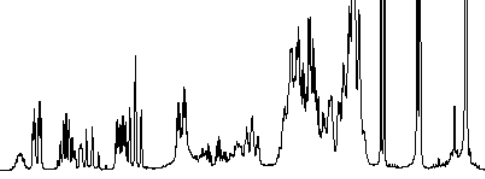

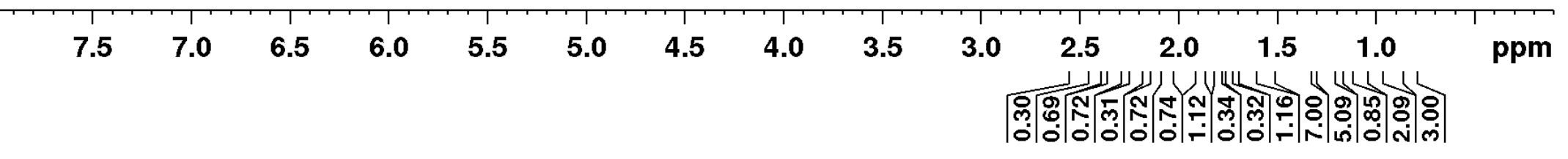


箱

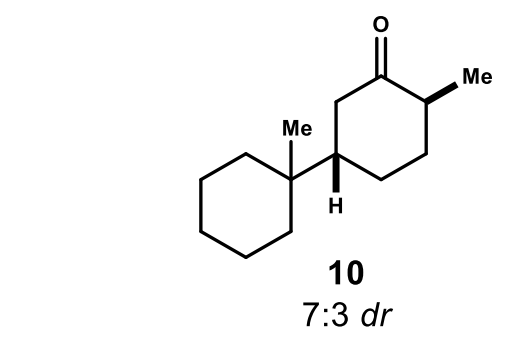

200

180

160

140

120

100

80

60

40

20

ppm
Current Data Parameters

NAME

EXPNO
PROCNO

F2 - Acquisition Parame Date_ 20200824 Time

INSTRUM

PROBHD

TD

SOLVENT

NS

DS

FIDRES

$\mathrm{AQ}$
$\mathrm{RG}$

RG

$\mathrm{DW}$

TE

TE
D1
D11
TDO

$====$
SFO1
NUC1

$\mathrm{N} 1$
$\mathrm{P} I \mathrm{C} 1$ av 600
$\mathrm{~mm}$ CPBBO BBzgdc 30

$\mathrm{CDCl} 3$

4
36231.883
0.552855 0.952855 2050
13.800

19.63
298.0

1.50000000 0.03000000 CHANNEL $\mathrm{f} 1===$ 150.9194080
$13 \mathrm{C}$ PLW1 64.00000000

$=======$ CHANNEL $\mathrm{f} 2===$

SFO2 600.1330010

NUC2 $1 \mathrm{H}$

Waltz16

PLW2 30.00000000

PLW12 12

F2 - Processing paramet

SI 65536

$\begin{array}{ll}\text { SF } & 150.9028085 \\ \text { WDW } & \end{array}$

WDW

LB $\quad 1.00$

GB

1.00 

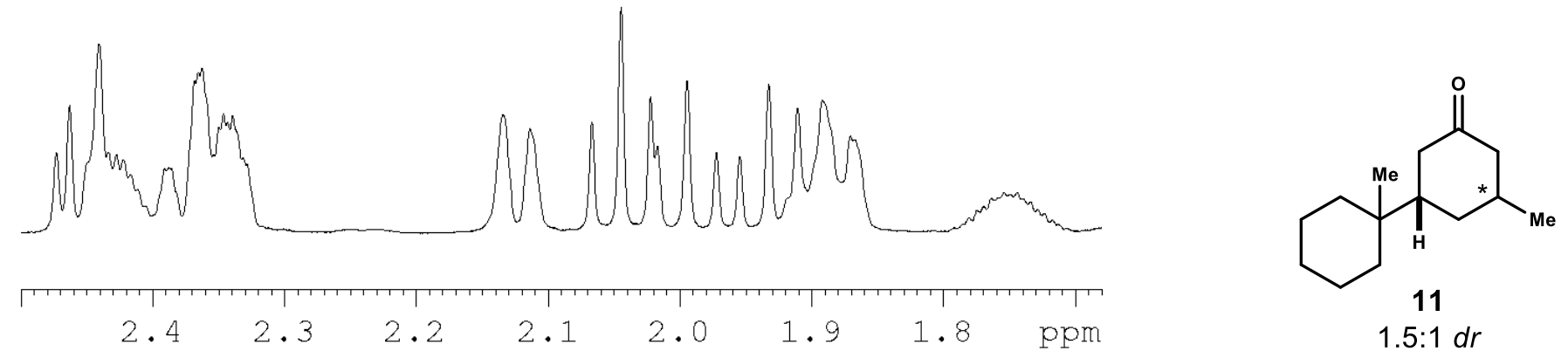

F2 - Acquisition Parame Date_r 20200825

\section{6}

1.5

1.3

1.2

How

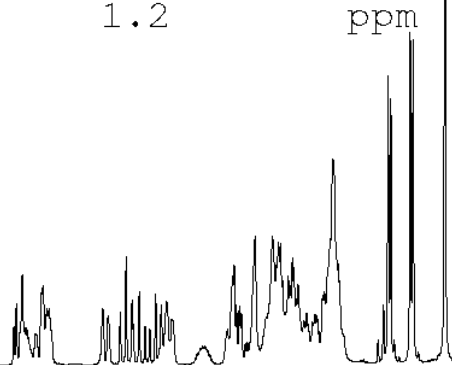

1.00

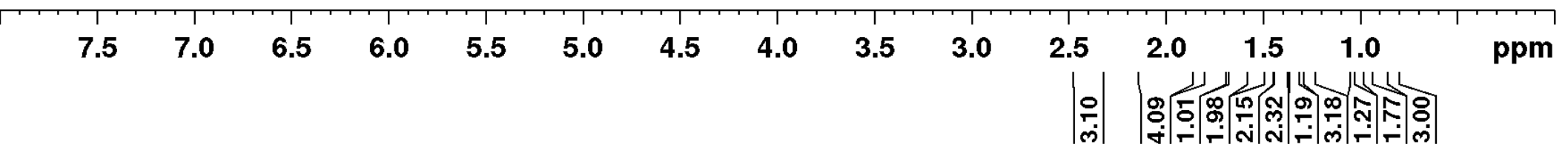


F2 - Acquisition Parane

Date

Time

IN⿴囗十)

PROBHD

TD

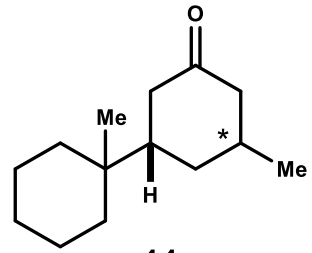

$1.5: 1 d r$

NS

DS

FIDRE

$\mathrm{AQ}$

RG

$\mathrm{DW}$

$\mathrm{DE}$

D1
D11

D11
TD0

$====$
$\mathrm{SFO1}$

SFO1
NUC1

P 1

PLW1

$====$
SFO2
NUC2

CPDPRG [2

PCPD

PLW12

20200825
10.04 10.04 mm CPBBO BBzgdc 30
65536

$\mathrm{F} 2$

SI

SE
WDW
SSB
TB

LSB
GB

GB
PC

4

36231.883

0.9043968

2050

13.800
19.63

298

1.0000000

0.03000000
1

CHANNEL $\mathrm{f1}===$

$13 \mathrm{C}$
150.9

64.00000000

CHANNEL $f 2===$

600.1330010

$1 \mathrm{H}$
waltz16
80.00

30.00000000

0.37154001

rocessing paramet

150.9028085

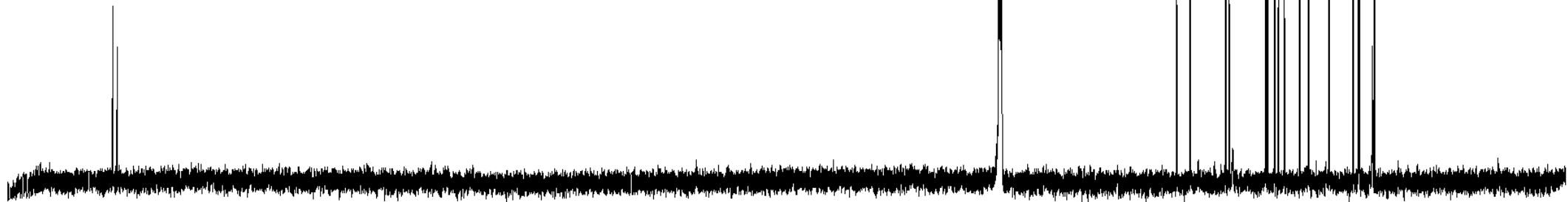

1.00

1.00

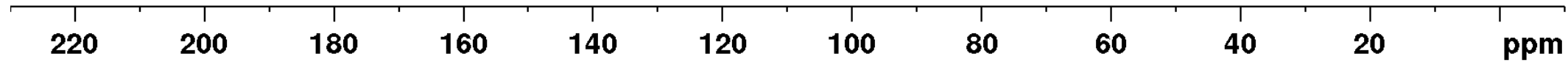



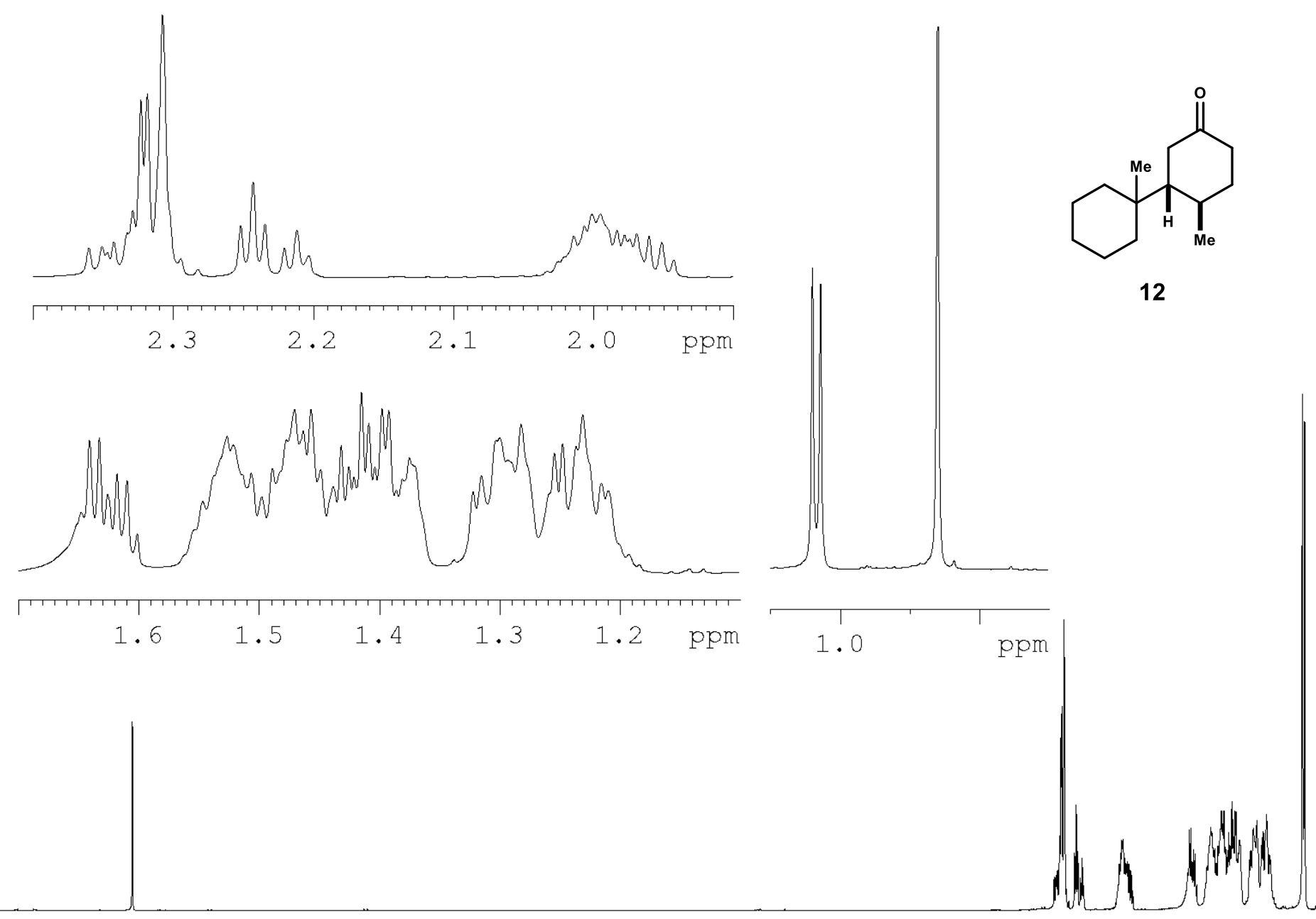
Tate Acquisition Parame

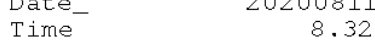
INSTRUM 5 aV600 PROBHD 5 Jm CPBBO BBZULPRO TD

SOLVEN

NS

DS

SWH $\quad 9615.385$ AQ $\quad 5.0998478$ RQ RG $\mathrm{DE}$ TE
D1 TDO

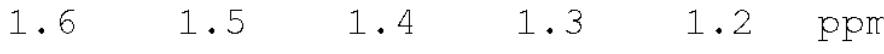



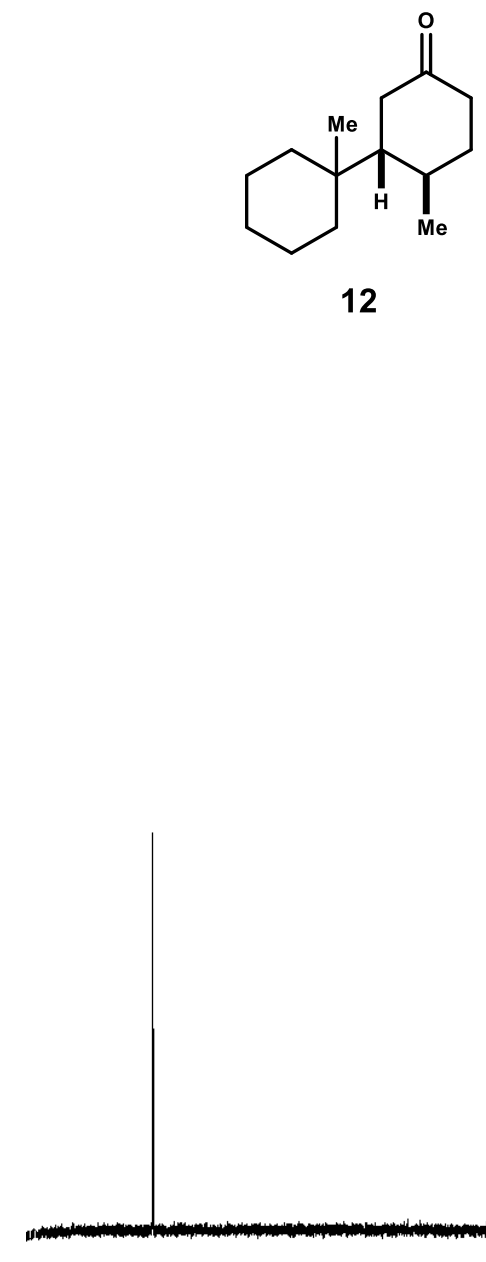

Current Data Parameters NAME

EXPNO

F2 - Acquisition Parane Date

Time

mm $\mathrm{CPBBO} \mathrm{BB}-$

zgdc 30

SOLVENT

NS

$\begin{array}{lr}\text { DS } & 4 \\ \text { SWH } & 36231.883\end{array}$

AO $\quad 0.552855$

RG

DW

$\mathrm{DE}$

D1

D11

$====$

$\mathrm{SEO} 1$
NUC1

P1

PLW1

2050

13.800

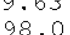

1.50000000 0.03000000

CHANNTI 11

150.9194080

$13 \mathrm{C}$
10.10

64.00000000

$=======\begin{array}{r}\text { CHANNEL } f 2=== \\ \text { SFO2 }\end{array} \quad=00.1330010$ 600.1330010
$1 \mathrm{H}$

CPDPRG [2 waltz16

PCPD2 80.00

PLW2 30.00000000

PLW12

0.37154001

F2 - Processing paramet

SI 65536

$\begin{array}{ll}\text { SF } & 150.9028085 \\ \text { WDWN } & \end{array}$

SSB

0

1.00

GB

1.00 

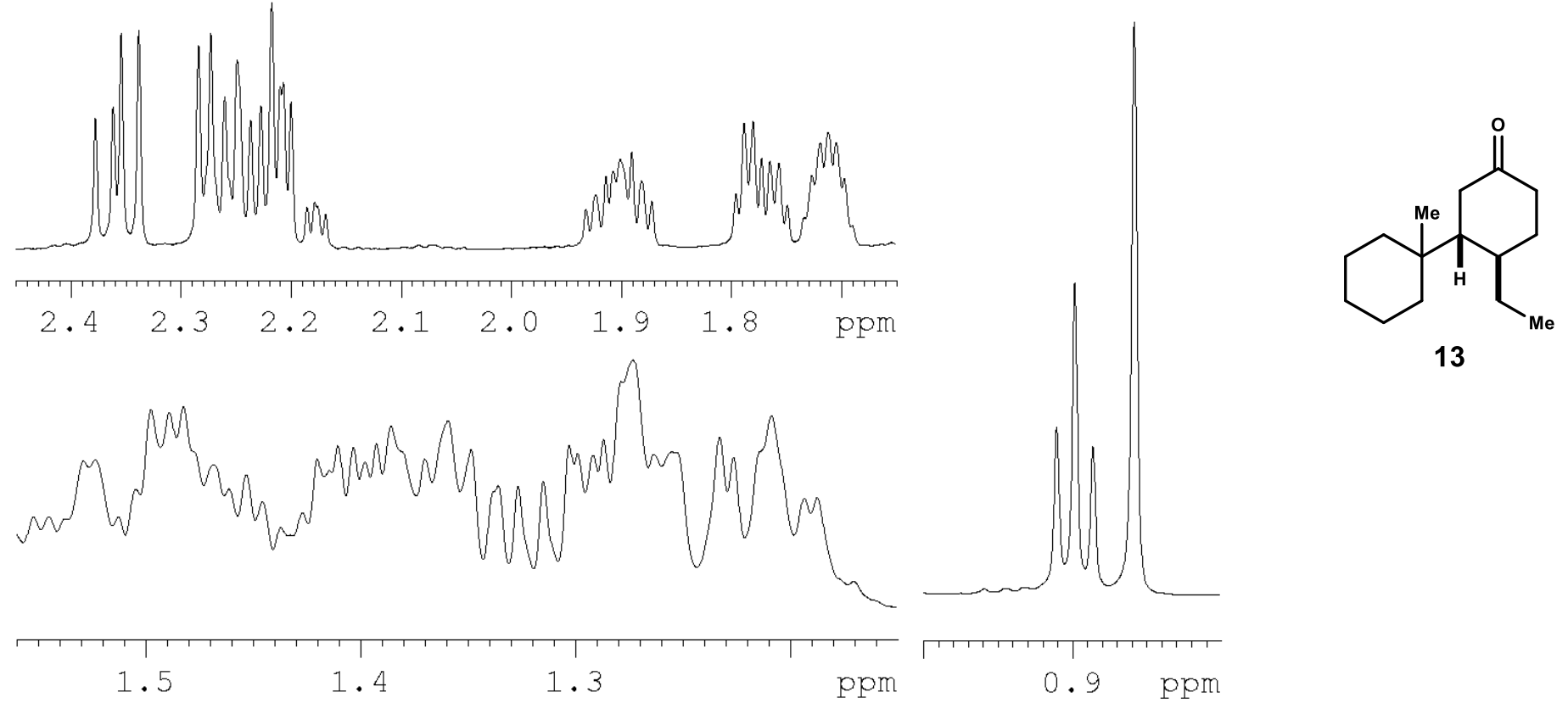

13

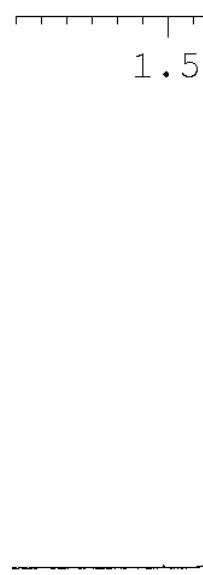

1.3

ppm

$0.9 \mathrm{ppm}$
F2 - Acquisition Parame Date

Iime

$5 \mathrm{~mm}$ CRBBO

PULPROG $\quad \mathrm{zg} 30$

TD $\quad 98074$

SOLVENT

NS

SWH
FIDRES

$\mathrm{FID}$
$\mathrm{AQ}$

RG

DW

DE

D1
TDO

$====$

SFO1

P1

PLW1

16
2
9615.385

0.098042 5.0998478

52.000

14.23

0.10000000 600.1342009 $1 \mathrm{H}$

F2 - Processing paramet

SI 65536

$\begin{array}{ll}\mathrm{SF} & 600.1300354\end{array}$

WDW

600.1300354
EM

BB

0.30

PC

1.00

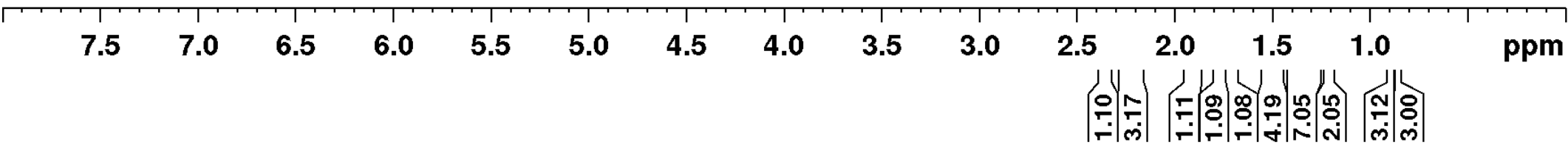




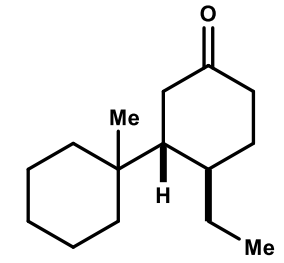

13

\begin{tabular}{|c|c|c|c|c|c|c|c|c|c|c|}
\hline $\begin{array}{c}1 \\
220\end{array}$ & 200 & $\begin{array}{c}18 \\
180\end{array}$ & 160 & 140 & $\begin{array}{c}1 \\
120\end{array}$ & 100 & 80 & 60 & 40 & 20 \\
\hline
\end{tabular}



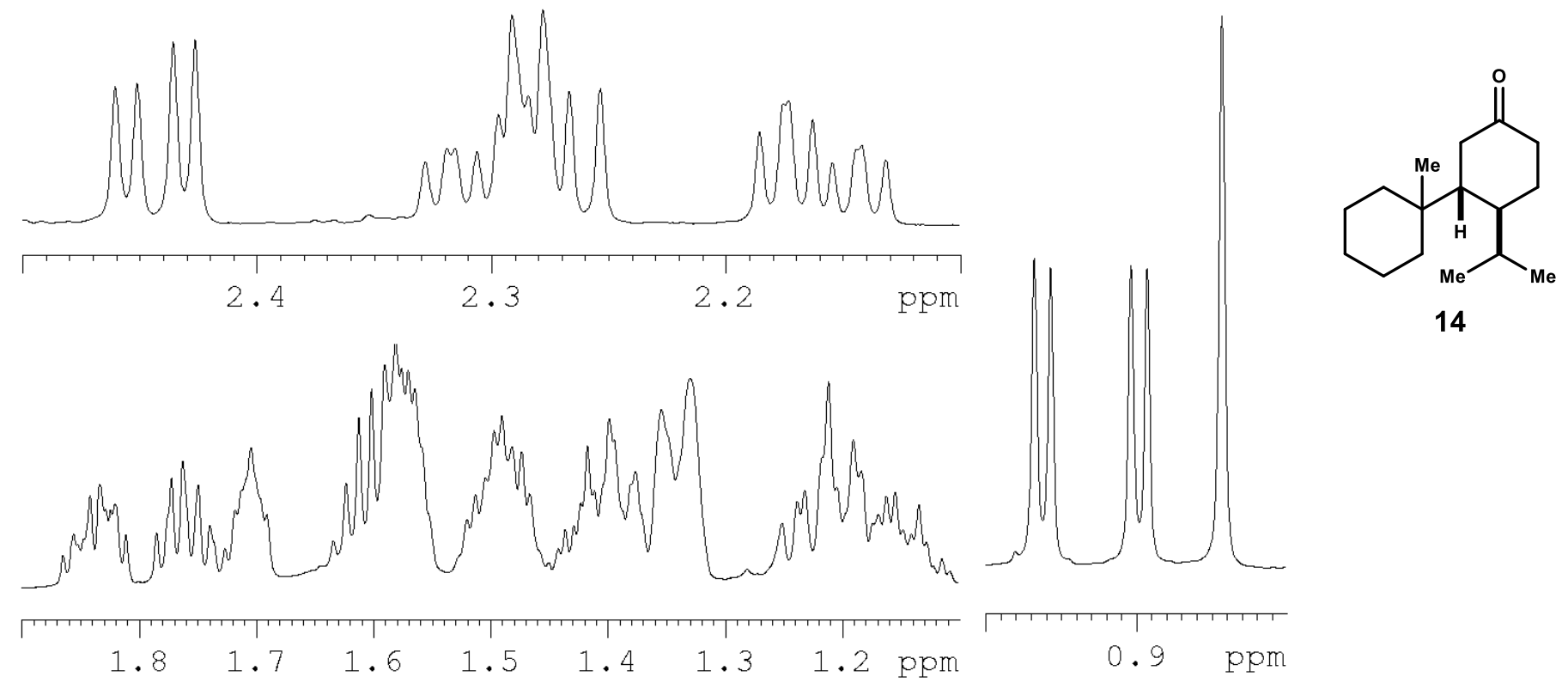

14

-9 ppm

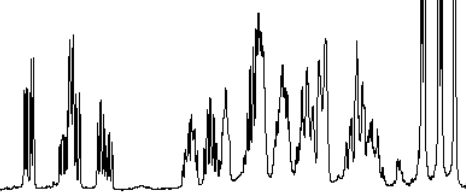

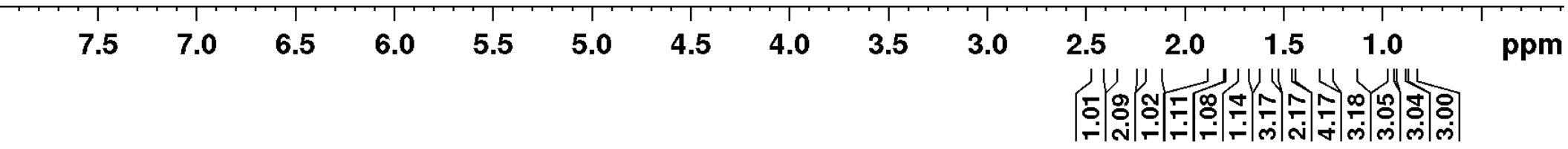




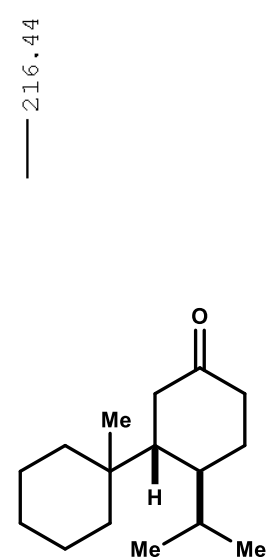

14

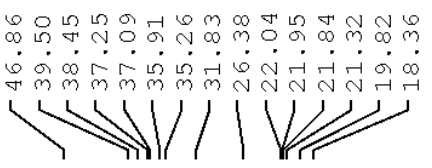

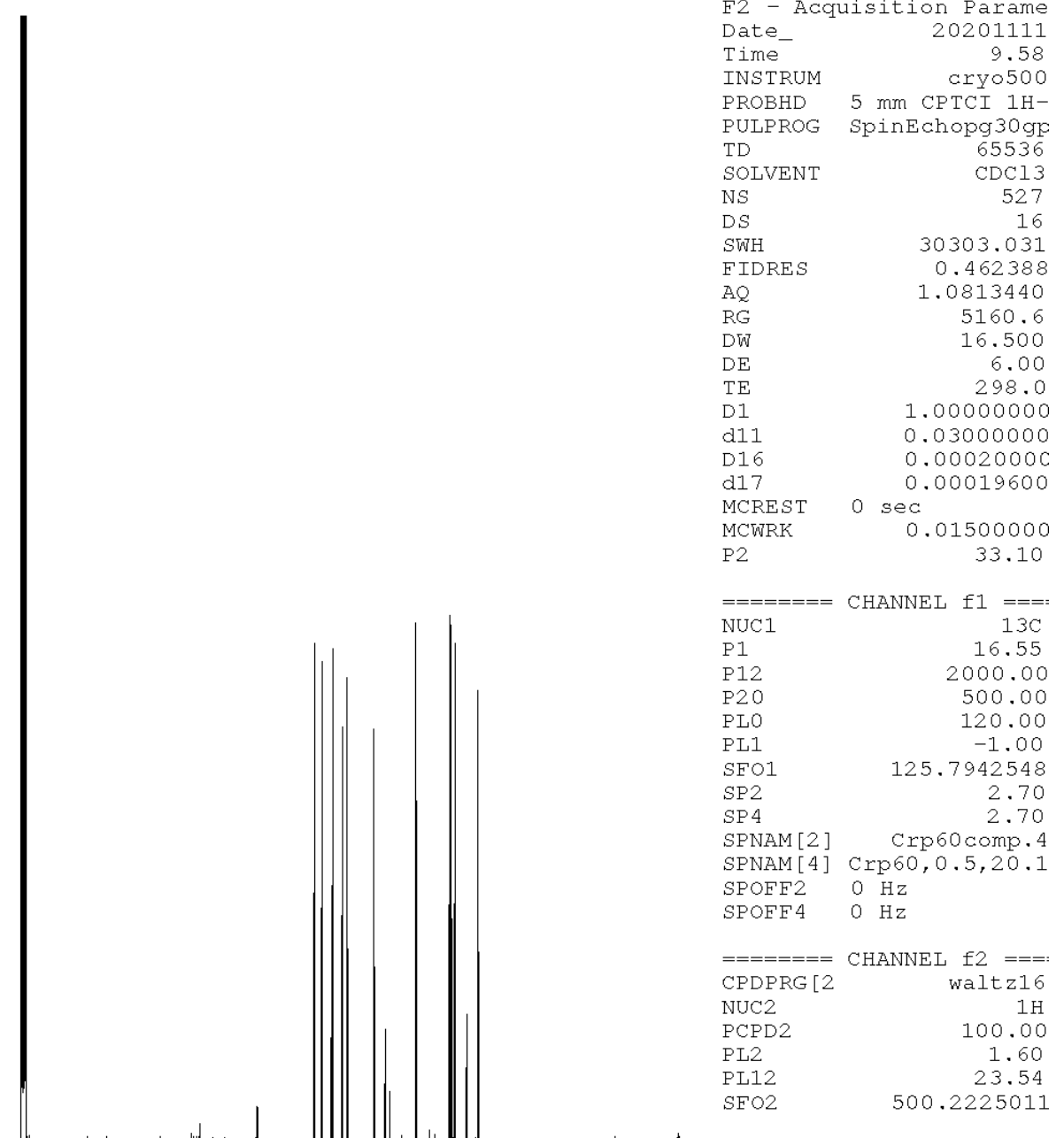

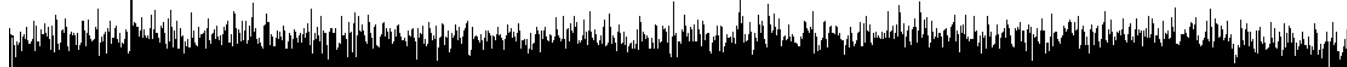

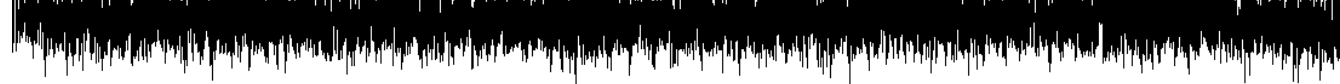

Grease

\begin{tabular}{|c|c|c|c|c|c|c|c|c|c|c|}
\hline 220 & 200 & 180 & 160 & 140 & 120 & 100 & 80 & 60 & 40 & 20 \\
\hline
\end{tabular}



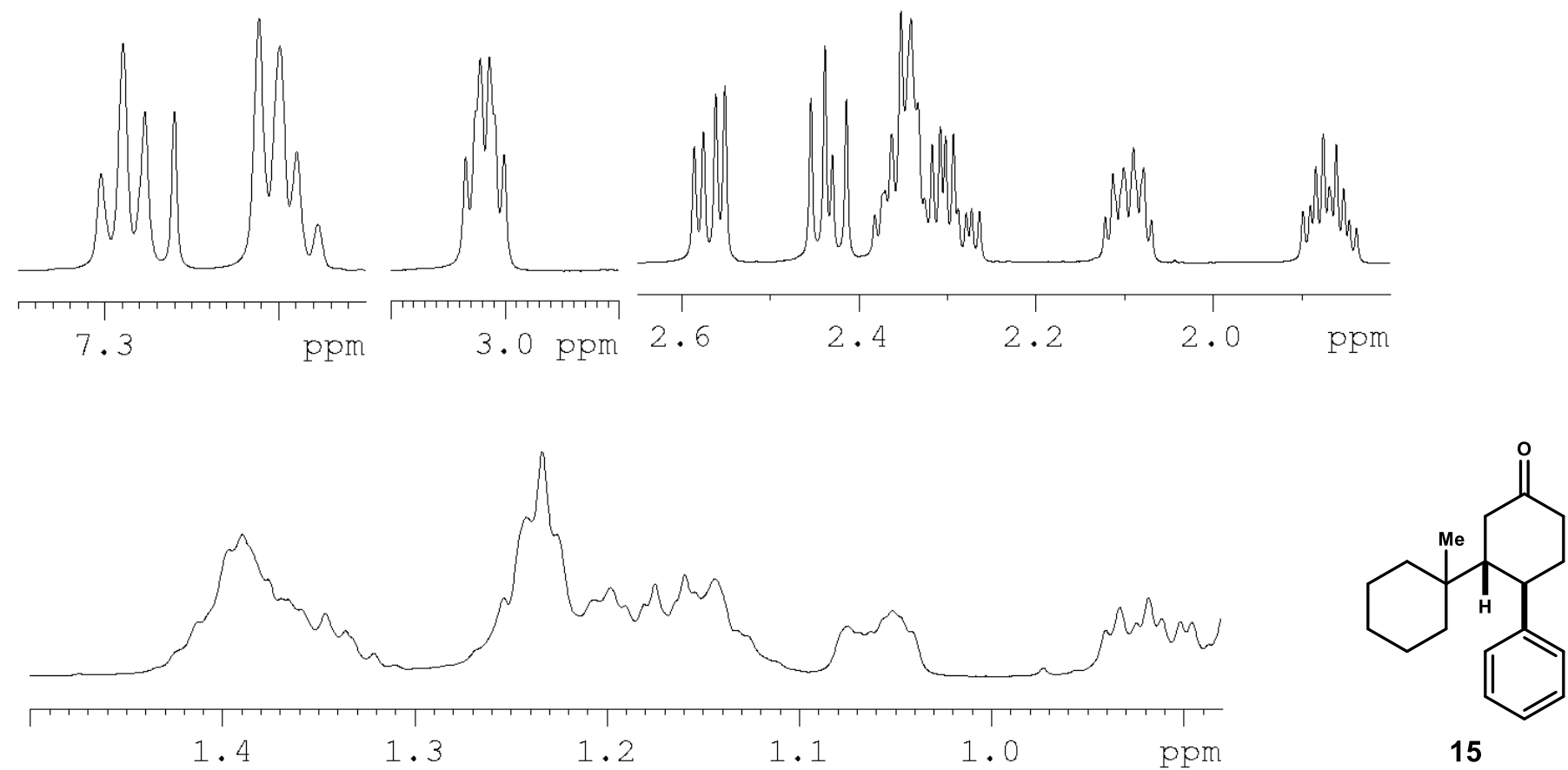

$$
\text { F2 - }
$$

Date

Time
INSTRUM

PROBHD

PUT

TD
SOLVENT

NS

DS

SWH $\quad 9615.385$

$\begin{array}{lr}\text { FIDRES } & 0.098042 \\ \text { AQ } & 5.0998478 \\ \text { RG } & 10\end{array}$

DW

$\mathrm{DE}$

D1

TDO

$====$
SFO1
NUC1

P1

PLW1

10
52.000

14.23
298.0

0.10000000

F2 - Processing paramet

$\begin{array}{lr}\text { SI } & 65536 \\ \text { SF } & 600.1300348\end{array}$

$\begin{array}{lr}\text { SF } & 600.1300348 \\ \text { WDW } & \text { EM }\end{array}$
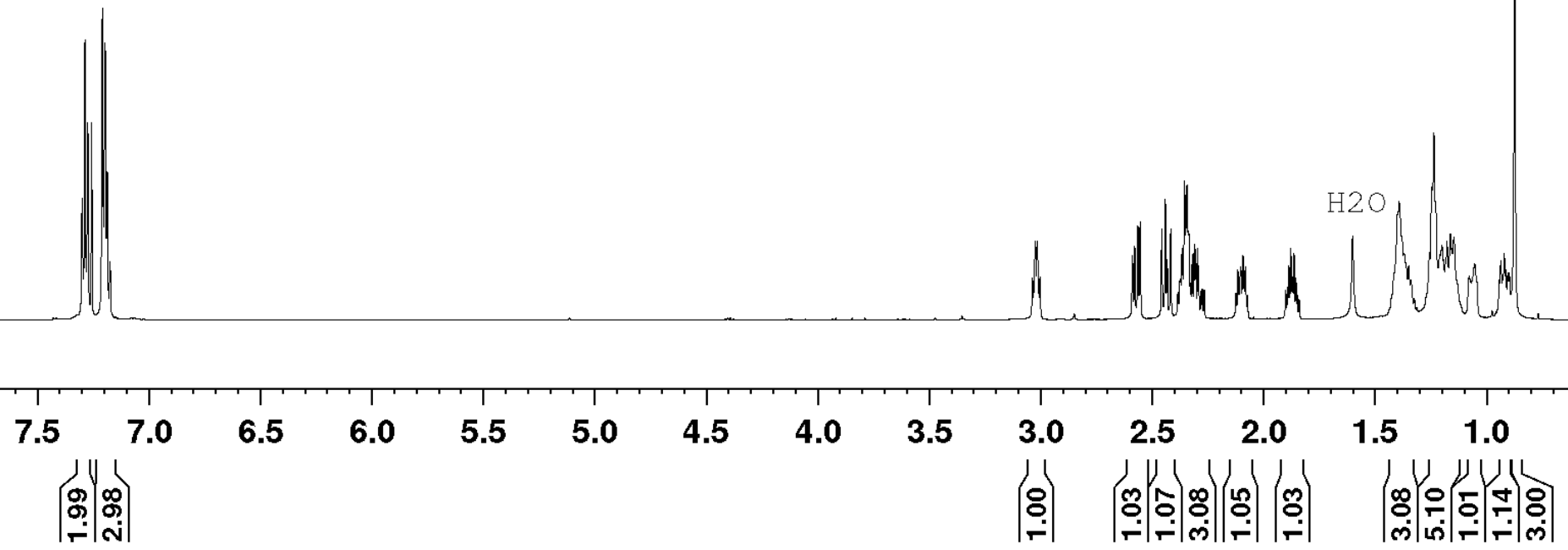


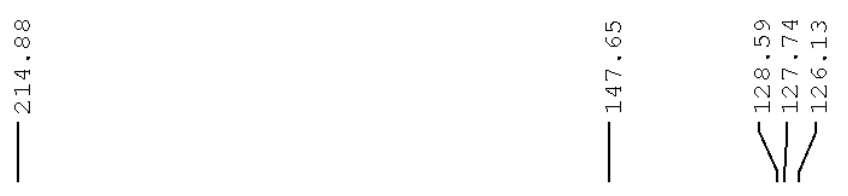

15

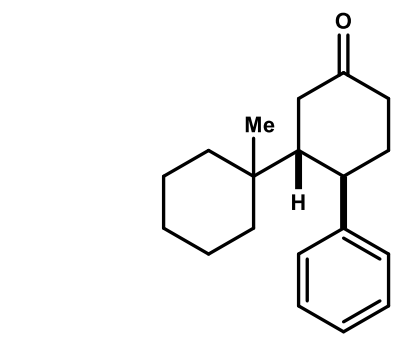

익

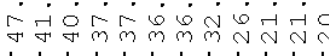

MWKIIVI
|
NAME

EXPNO

PROCNO

F2 - Acquisition Parame Deter

Date

Time

PROBHD

PUTPROG

TD

DS

DS

FIDRES

$\mathrm{AQ}$

RG

$\mathrm{DW}$

TE

D1

D11
TD0

$$
====
$$

$\mathrm{SFO}$

NUC1

P1
PLW1

\section{$==$}

$\mathrm{SFO} 2$

CPDPRG [2

PCPD2

PLPD2

PLW12

0.00000000 F2 - Processing paramet

SI

WD

SS

LB

GB
PC

20200901

av600 zgde 30
65536

zgdc 30
65536

560

4
36231.883

0.552855 0.9043968 2050
13.800

19.63
298.0

1.50000000 0.03000000

1

150.9194080

64.00000000

CHANNEL $\mathrm{f} 2==$ $1 \mathrm{H}$ 80.00 150.9028085 EM 1.00

1.00
$13 \mathrm{C}$
10.10

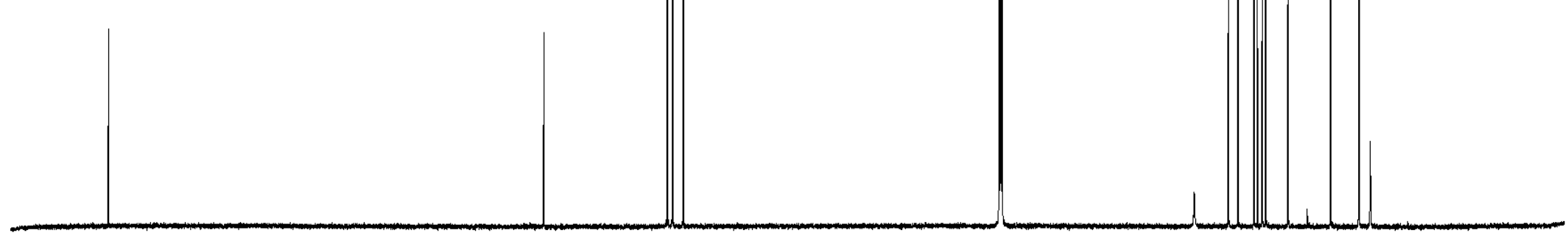

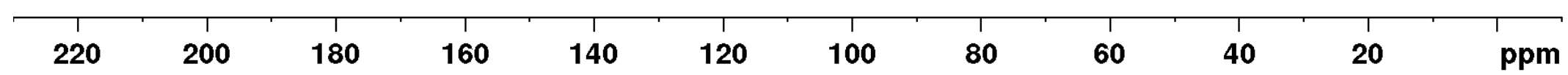



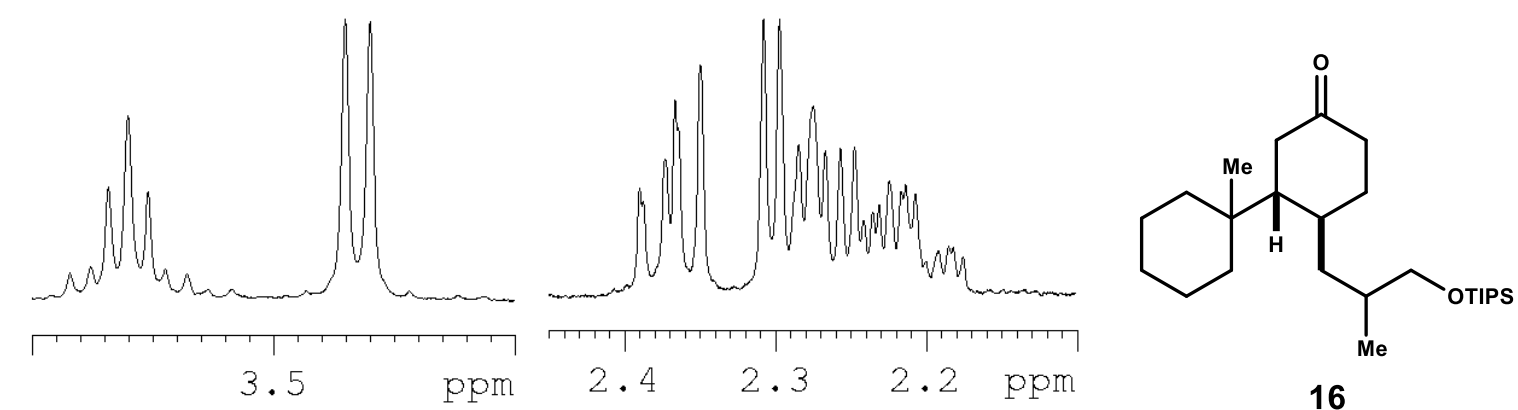

F2 - Acquisition Parame Date

Time

INSTRUM

PROBHD

TD

SOLVENT

NS

DS

FIDRES
AQ

RG

DW

$\mathrm{DE}$

$\mathrm{TE}$
$\mathrm{D} 1$

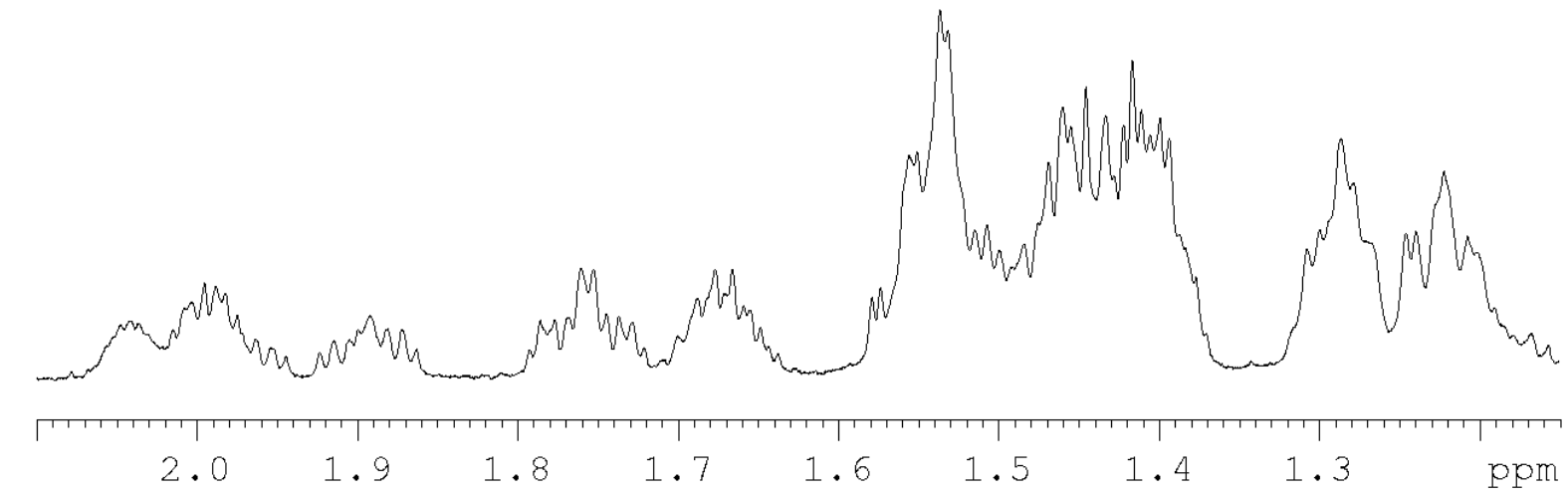

TDO

$===$

$\mathrm{SFO} 1$
NUC1

P1

7.32

$5 \mathrm{~mm}$ СPBBO $\mathrm{BB}-$

a $\mathrm{BB}-$

$\mathrm{zg} 30$
98074

PLW

9615.385

0.098042 5.0998478

52.000

14.23

0.10000000

CHANNET 11000 600.1342009

30.50

F2 - Processing paramet

SI 65536

SF $\quad 600.1300295$

WDW

$\begin{array}{lllllllll}2.0 & 1.9 & 1.8 & 1.7 & 1.6 & 1.5 & 1.4 & 1.3 & \text { ppm }\end{array}$

0.30

1.00

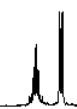

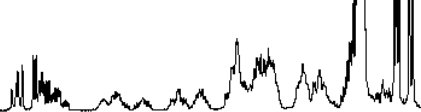

$\begin{array}{llllllll}7.5 & 7.0 & 6.5 & 6.0 & 5.5 & 5.0 & 4.5 & 4.0\end{array}$

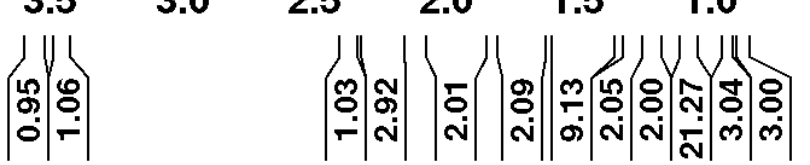

ppm 
F2 - Acquisition Parame

Date_ 20200819

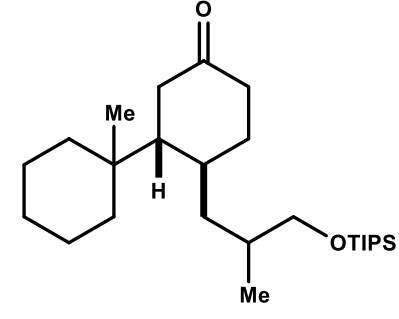

16
TD

SOLVEN

NS

DS

FIDRE

AQ

RG

DE

D1

D11

\section{$====$}

SEO1
NUC1

NUC1
$\mathrm{P} 1$

PLW1

$====$
SFO2
NUC2
CPDPR

CPDPRG [2

PCPD2

$\mathrm{PLW} 2$

F2 - Processing paramet SI

WF

SSB

LB

PC zgdc30
65536

CDC13

400

36231.883

0.552855

.9043968

2050
13.800

19.63
298.0

1.50000000 0.03000000

\section{CHANNEL $\mathrm{f1}==$}

150.9194080
$13 \mathrm{C}$

CHANNEL $f 2===$

$1 \mathrm{H}$

80.00

0.00000000

65536

150.9028085

1.00

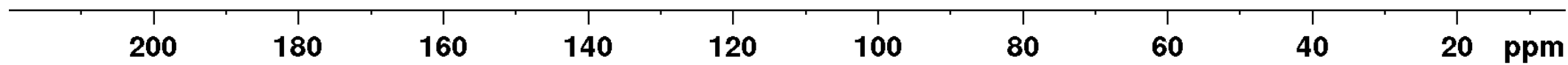




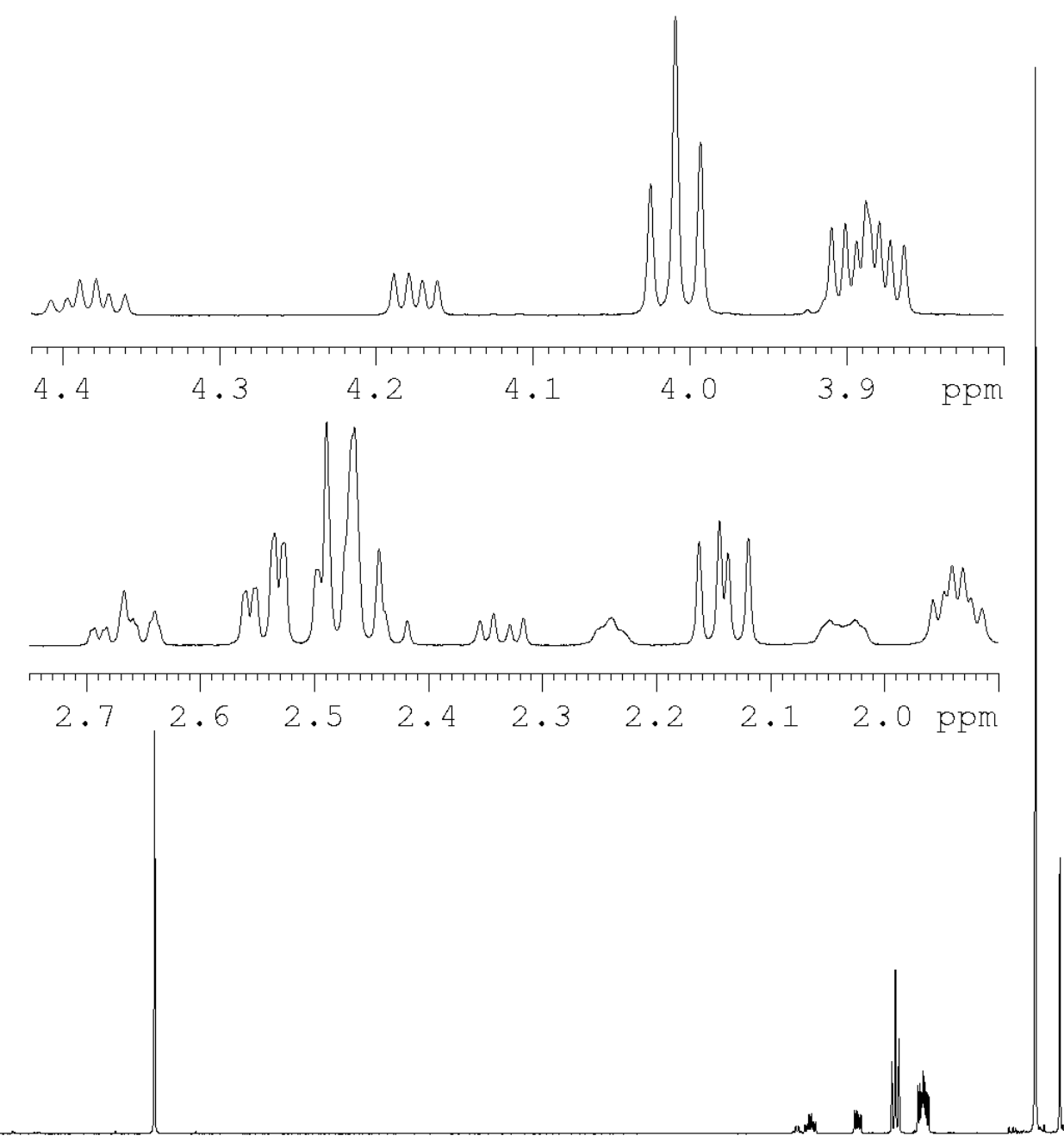

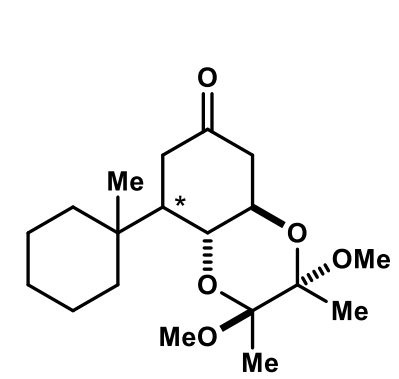

17

$\sim 4: 1 d r$

F2 - Acquisition Parame Date__ 20190607 20196076
TNSTRUM $\quad 16.360$

PROBHD $5 \mathrm{~mm} \mathrm{CPBBO} \mathrm{BB}-$

PROBHD
PULPROG

$\mathrm{zg} 30$
98074

TD
SOLVENT $\quad 98074$

NS
DS

SWH $\quad 9615.385$

FIDRES $\quad 0.098042$ AQ $\quad 5.0998478$

RG

DW

TE

D1
TDO

$===$

$\mathrm{SFO1}$

NuC

P1
PLW1

52.000
13.70

0.10000000

CHANNEI 1 fNEL f1 $===$
600.1342009

12.00
$\quad 30.00000000$

F2 - Processing paramet

SI $\quad 65536$

WF

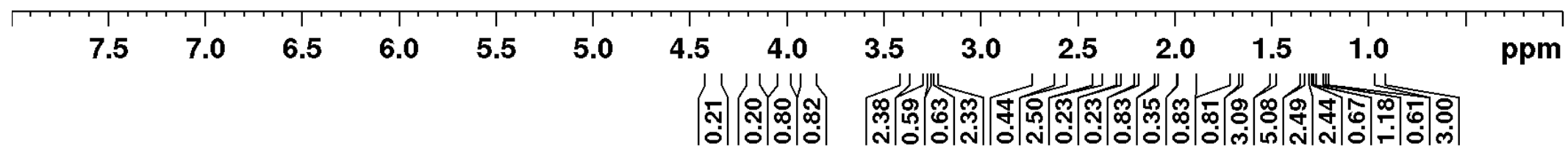


20190608
13.09

av600

PROBHD $5 \mathrm{~mm}$

zgdc 30

TD

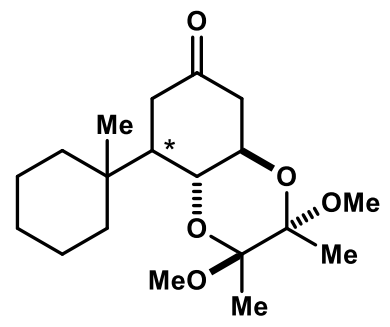

17

$\sim 4: 1 d r$

\begin{tabular}{rrrrr|r|r|rrr|r}
\hline 200 & 180 & 160 & 140 & 120 & 100 & 80 & 60 & 40 & 20 & ppm
\end{tabular}



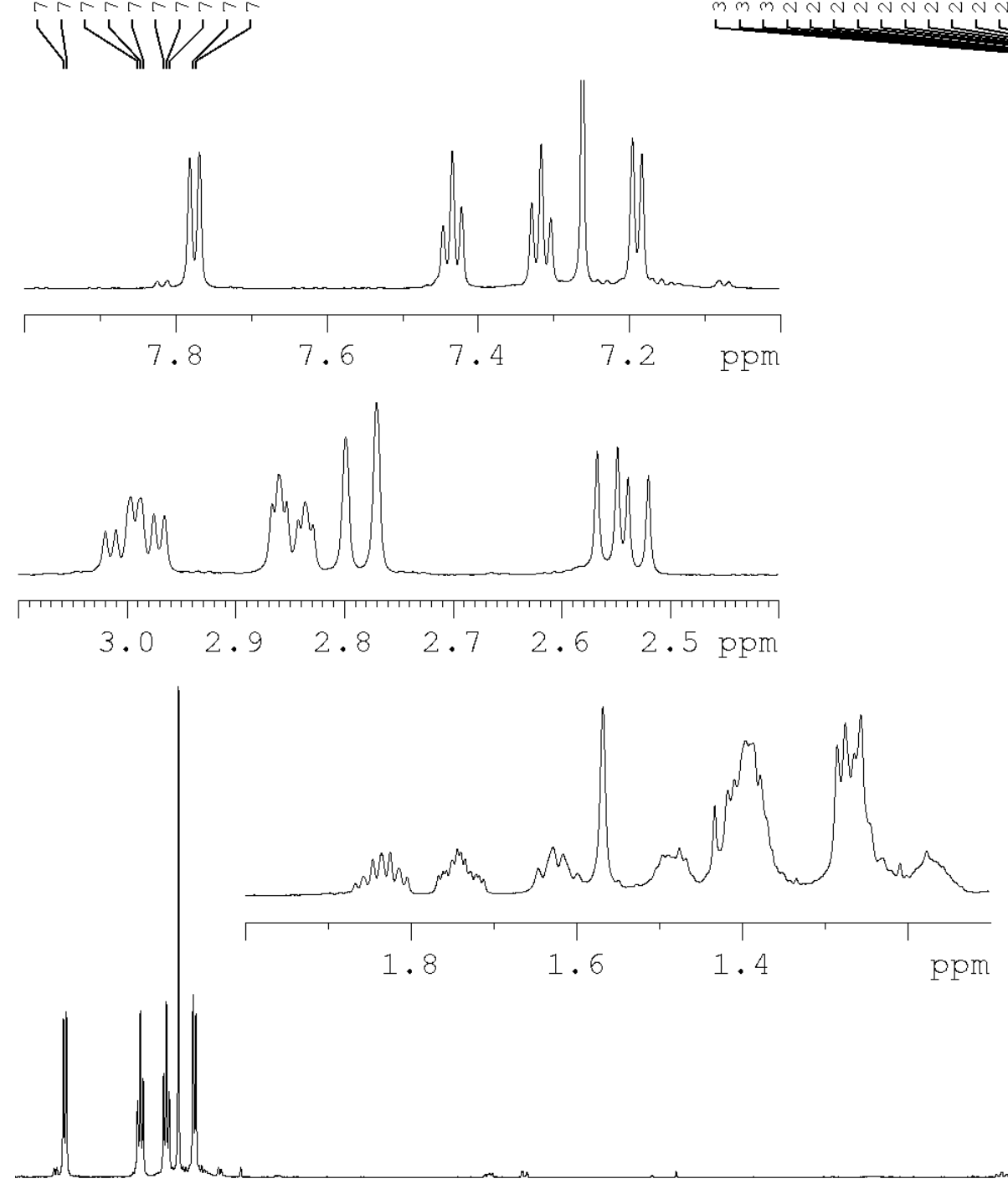
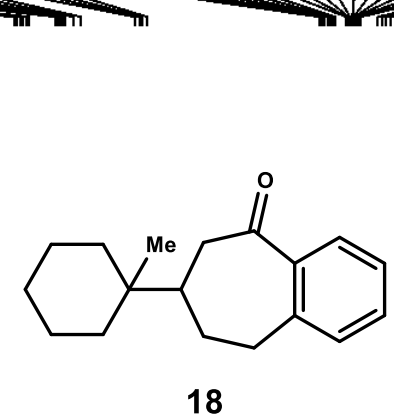

18
Current Data Parameters NAME EXPNO
PROCNO F2 - Acquisition Parame Date_

Time PROBHD $\mathrm{zg} 30$
98074 SOLVENT NS SWH FIDRES $\quad 9615.385$ $\quad 0.098042$ AQ $\quad 5.0998478$ DW

TE

D1
TDO

$====$ $\mathrm{SFO1}$ NUC1

P1
PLW1

$$
52.000
$$$$
14.23
$$

0.10000000 1 $1 \mathrm{H}$
9.50

F2 - Processing paramet SI 65536 SF $\begin{array}{llr}\text { WDW } & \\ \text { SSB } & 0 & 0.30\end{array}$

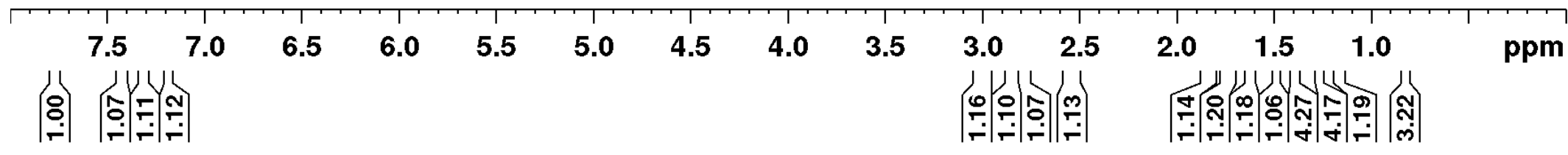



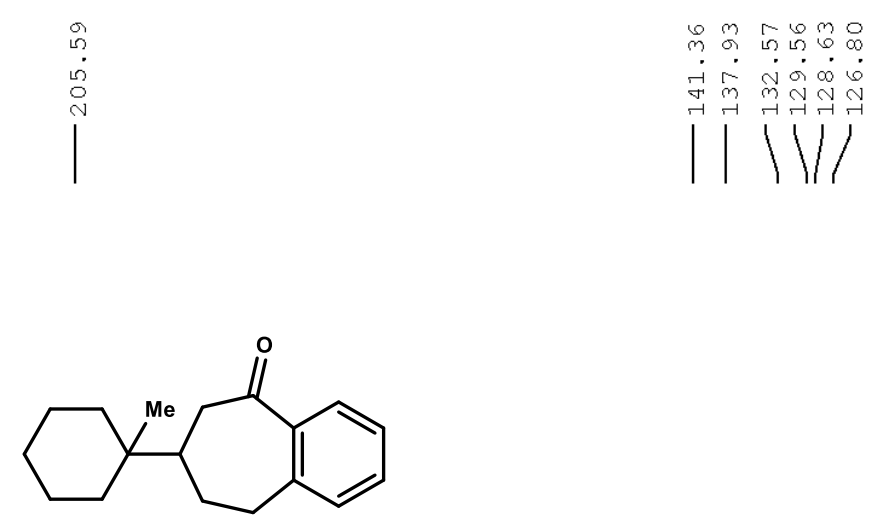

18

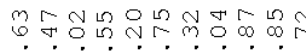

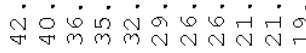
IIVIIVVI

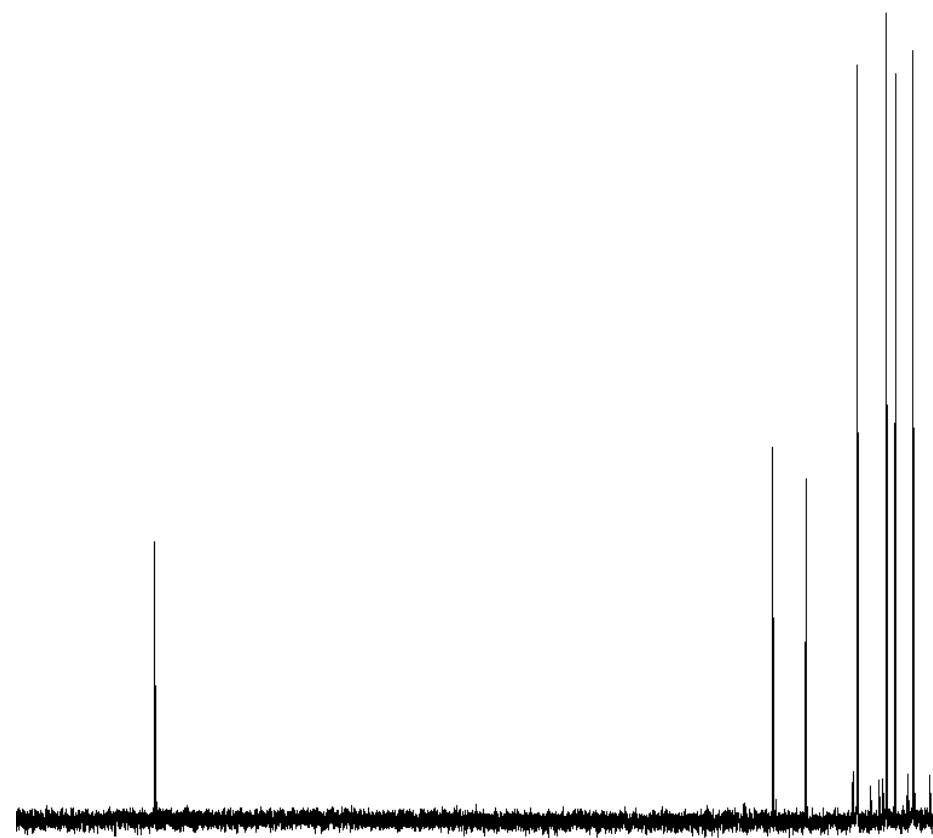

Current Data Parameters NAME

EXPNO Date

Time

INSTRUM

PROBHD

TD

SOLVENT

NS

DS

FIDRE

AQ

RW

$\mathrm{DE}$

D1

D11

$====$
SFO1

SFO1
NUC1

NUC1
P1

PLW1

$====$

$\mathrm{SFO} 2$

NUC2
CPDPRG [2

PCPD 2

PLW1

0.37154001

F2 - Processing paramet

SI 65536

WDW

LB

GB

PC

150.9028085

0

1.00

1.00

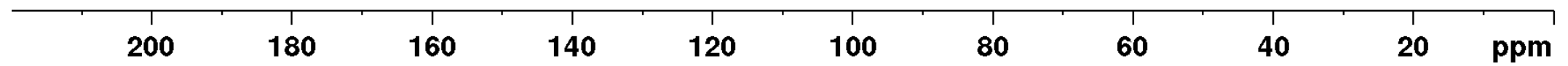



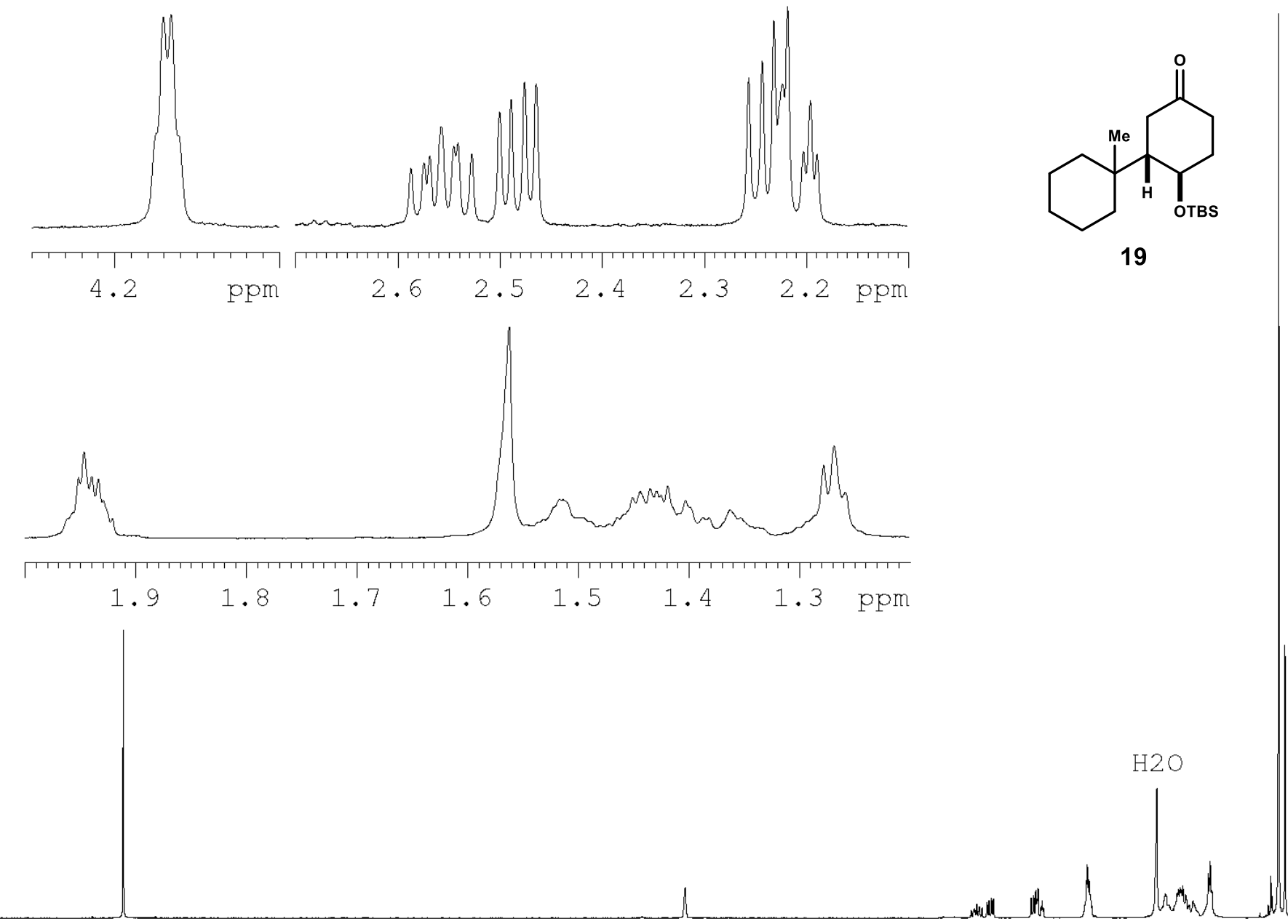

F2 - Acquisition Parame Date_ 20200314 Time 14.38 PROBHD $5 \mathrm{~mm}$ CPBBO BBPROBHD
PULPROG TD
TD SOIVTT

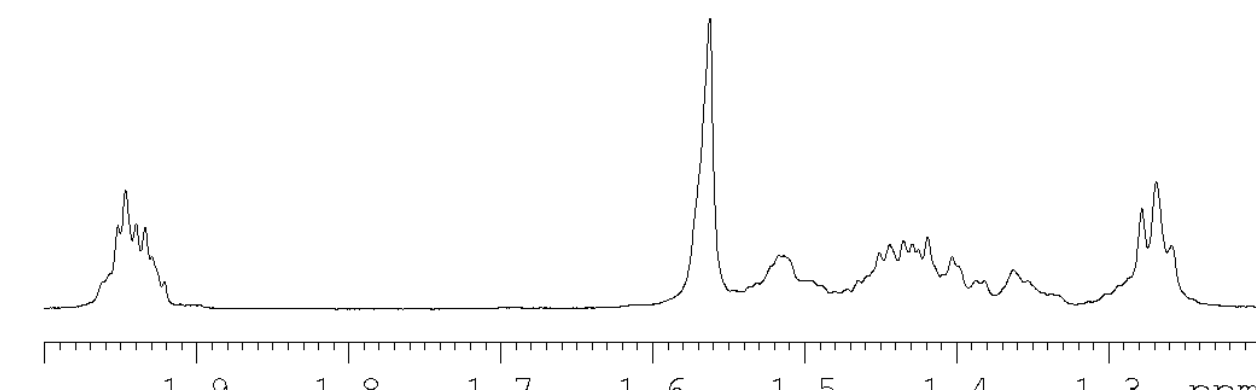

\section{SFO1}

NUC1

P1

PLW1

CHANNEL $\mathrm{f} 1===$

NNEL $\mathrm{fl}===$
600.1342009
$1 \mathrm{H}$

9.50 SI $\quad 65536$ SE

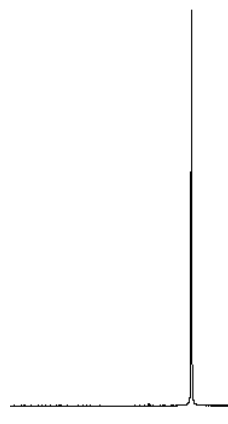

\section{1}

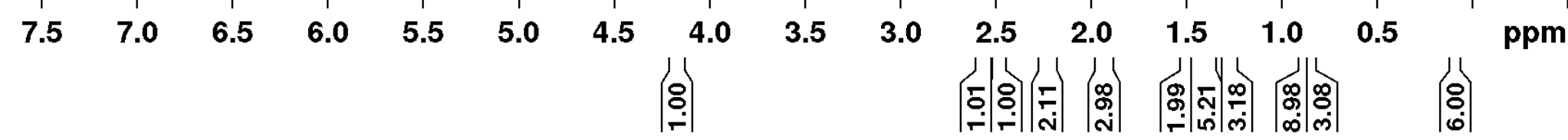


19
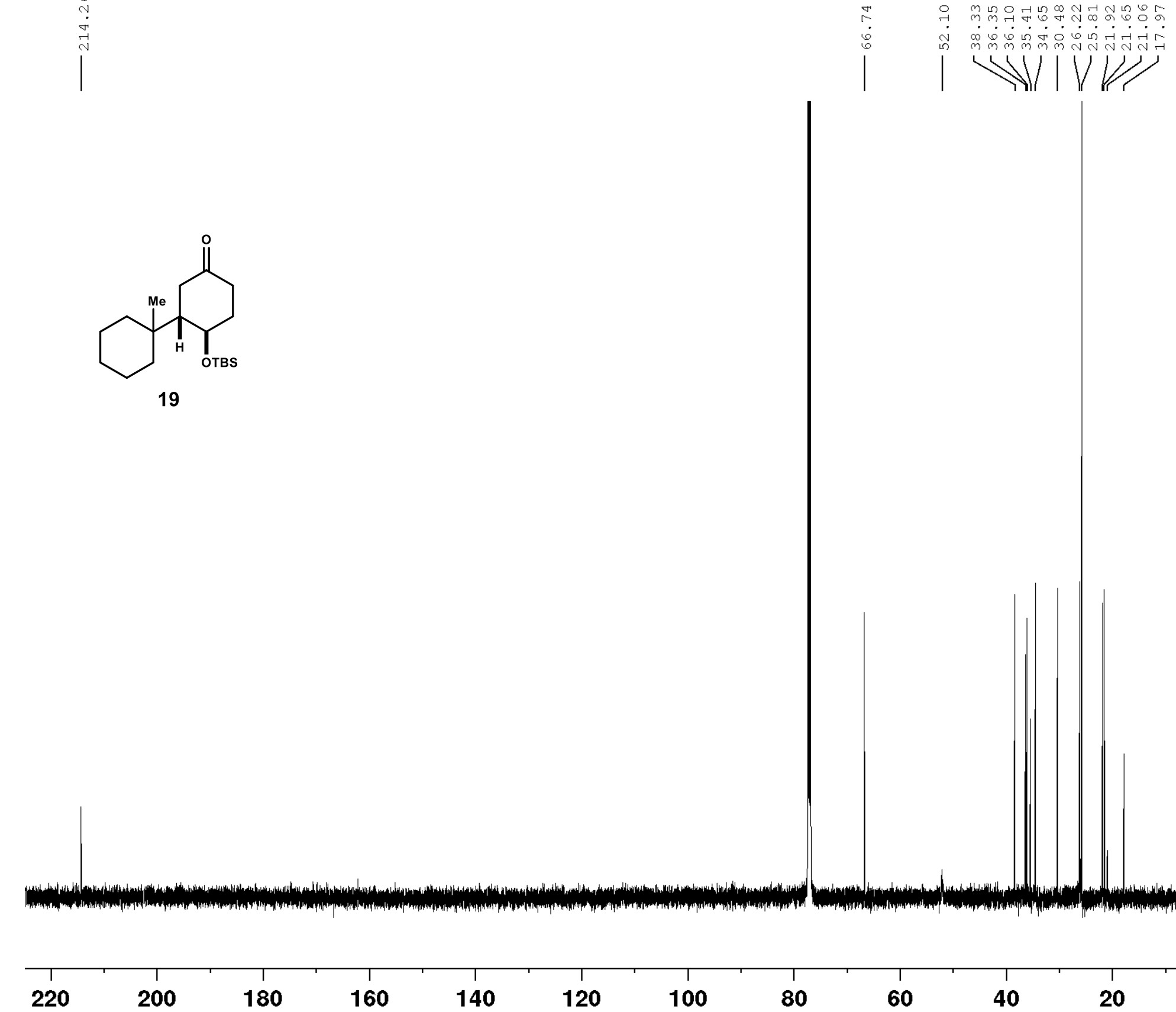

180

160

140

120
80

60

40

20

ppm

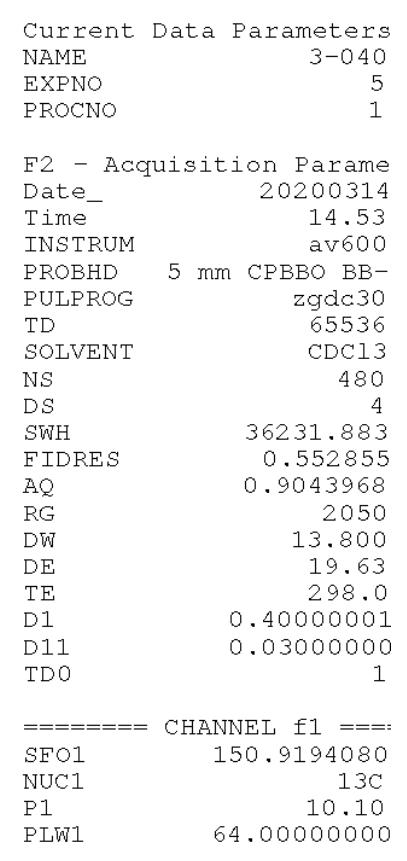

$====$
SFO2

CHANNEL $12===$

CPDPRG [2 waltz16

PCPD2 30.0000000

PLW12

0.37154001

F2 - Processing paramet

SI $\quad 65536$

SF $\quad 150.9028085$

LB

GB

$\mathrm{PC}$

o

1.00

1.00 

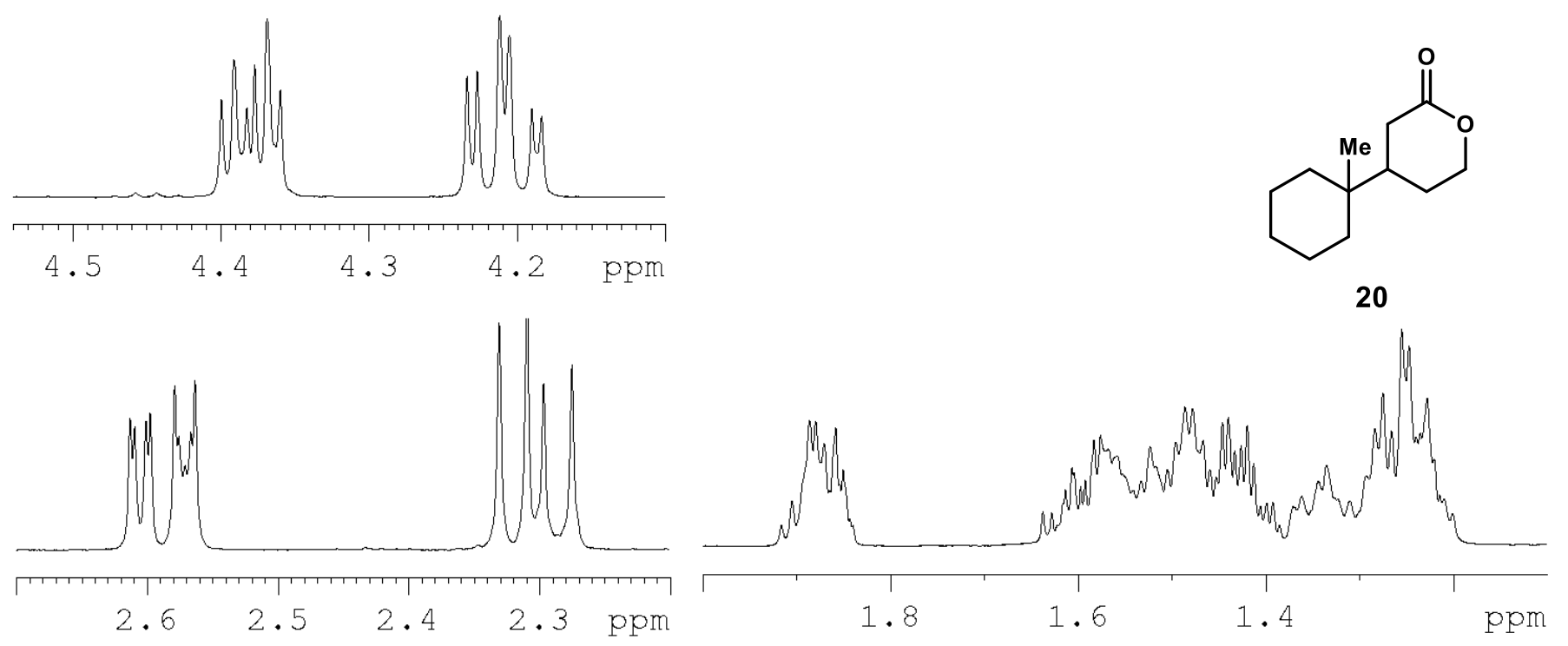

P-2-213-1H
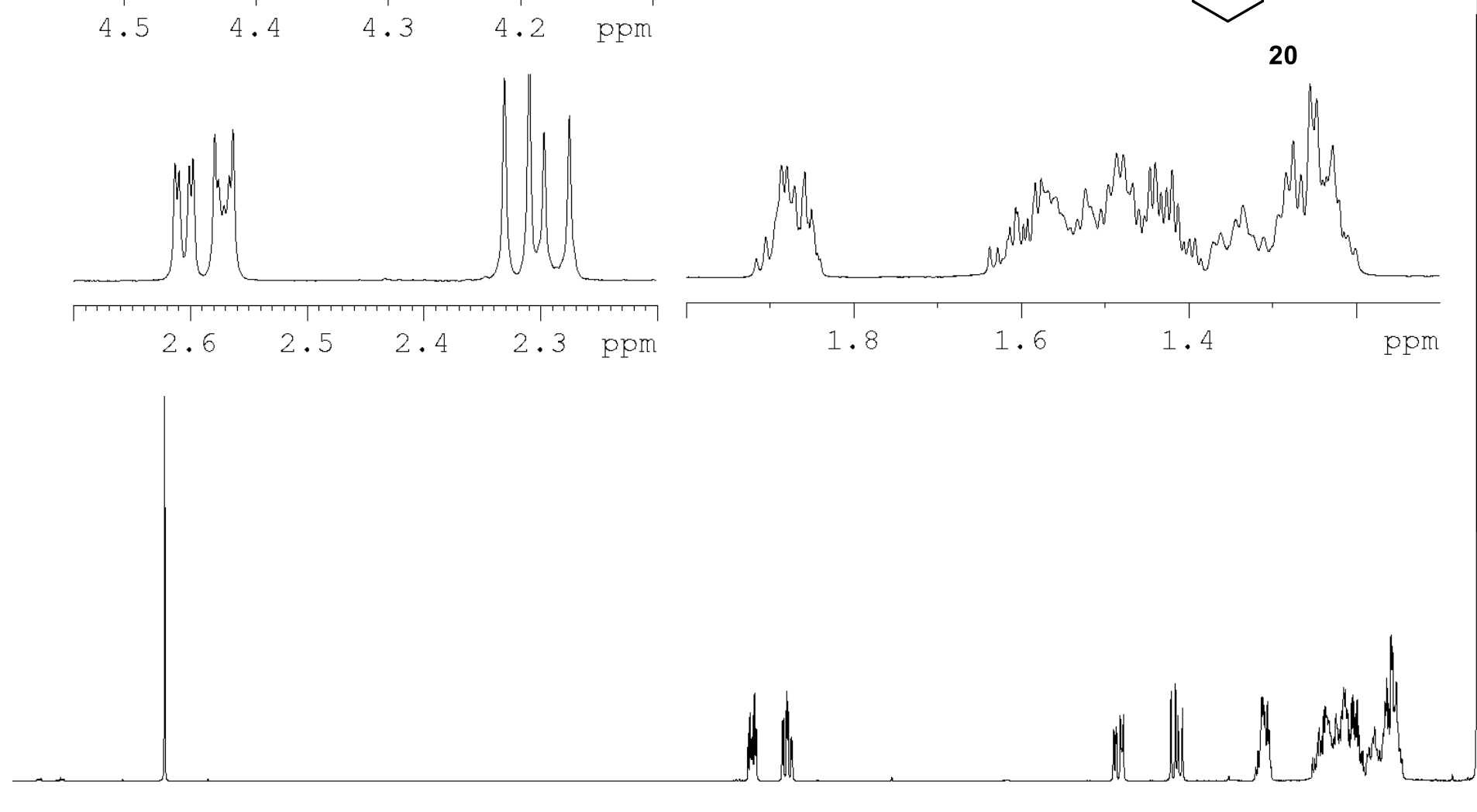

F2 - Acquisition Parame Date_

Time

$5 \mathrm{~mm}$ CPTCI $1 \mathrm{H}$

PUI PROC

TD

SOLVEN

NS

DS

FIDRES

AQ

DW

DE

D1

MCREST

MCWRK

$\mathrm{zg} 30$
81728

$====$

NuC1

P1
PL1

2
8012.820

0.098043 5.0998273

6.3
62.400

6.00
298.0

0.10000000 sec 0.01500000

SFO

CHANNEL $1===$
$1 \mathrm{H}$
7.50

500.2235015

F2 - Processing paramet

SI 65536

$S F$

\section{$\begin{array}{lllllllllllllll}7.5 & 7.0 & 6.5 & 6.0 & 5.5 & 5.0 & 4.5 & 4.0 & 3.5 & 3.0 & 2.5 & 2.0 & 1.5 & 1.0 & \mathrm{ppm}\end{array}$

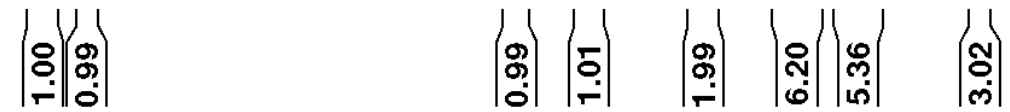




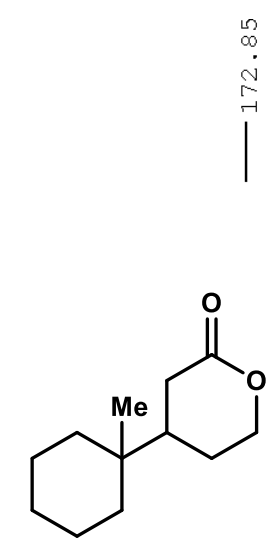

20
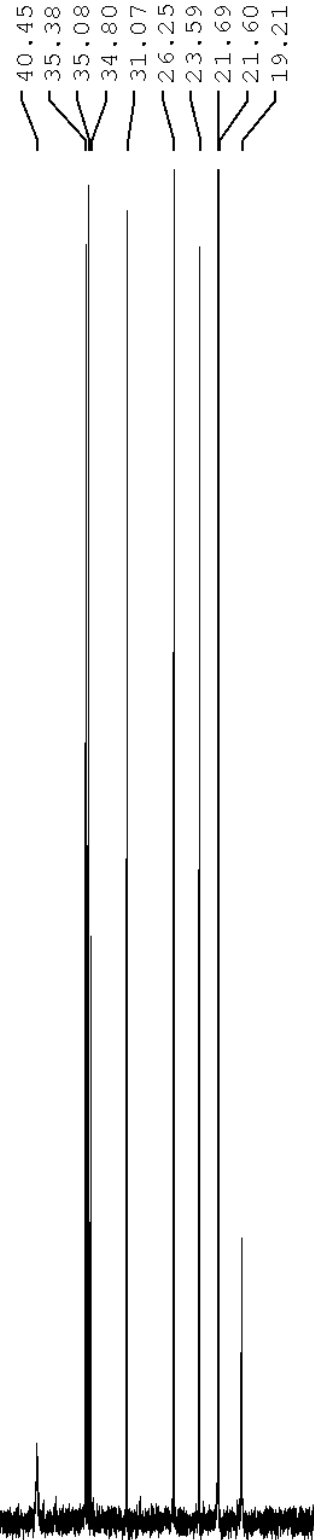

Current Data Parmeters

NAME

PROCNO

F2 - Acquisition Parame Date

Time

INSTRUM

PROBHD

TD

SOLVENT

NS

DS

FIDRES

$\mathrm{AQ}$

RG
DW

DE

D1

D11

$$
====
$$

$\mathrm{SFO1}$

NUC

P1

PLW1

SFO2

$\mathrm{SFO} 2$

CPDPRG [2

PCPD2

PLW2

F2

S2

SI

WDW

LB

GB
PC

$S P-2-213-13 \mathrm{C}$

1

20190605 9.12
av 600 $5 \mathrm{~mm}$ CPBBO BBzgda 30 CDC13 303

36231.883 0.552855 0.9043968 2050
13.800 19.63 0.40000001 0.03000000

(c) 150.9194080 $13 \mathrm{C}$ 64.00000000 HANNT2 == $1 \mathrm{H}$
waltz 16 30.00000000 0.63095999 Processing paramet 150.902536

1.00

1.00

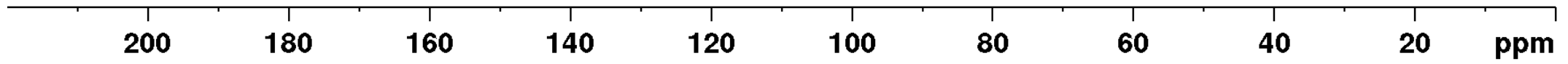



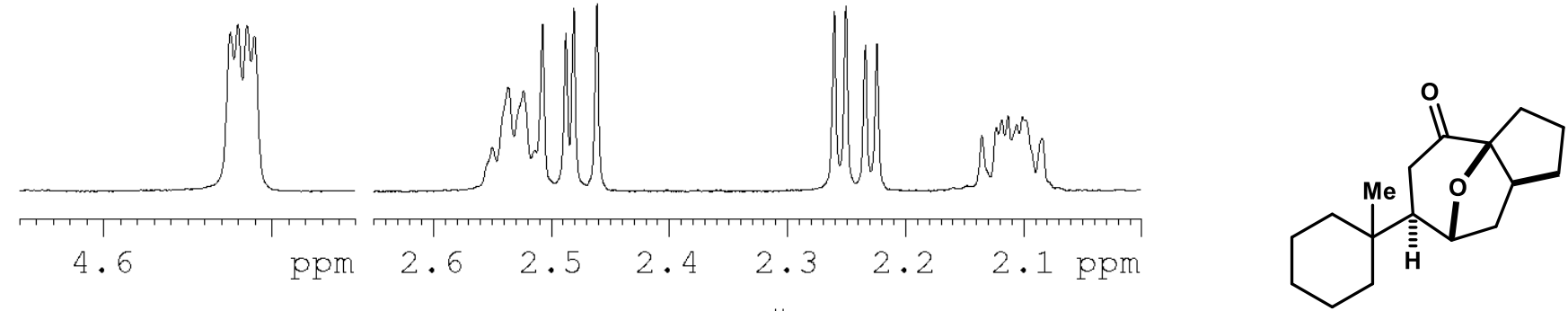

21

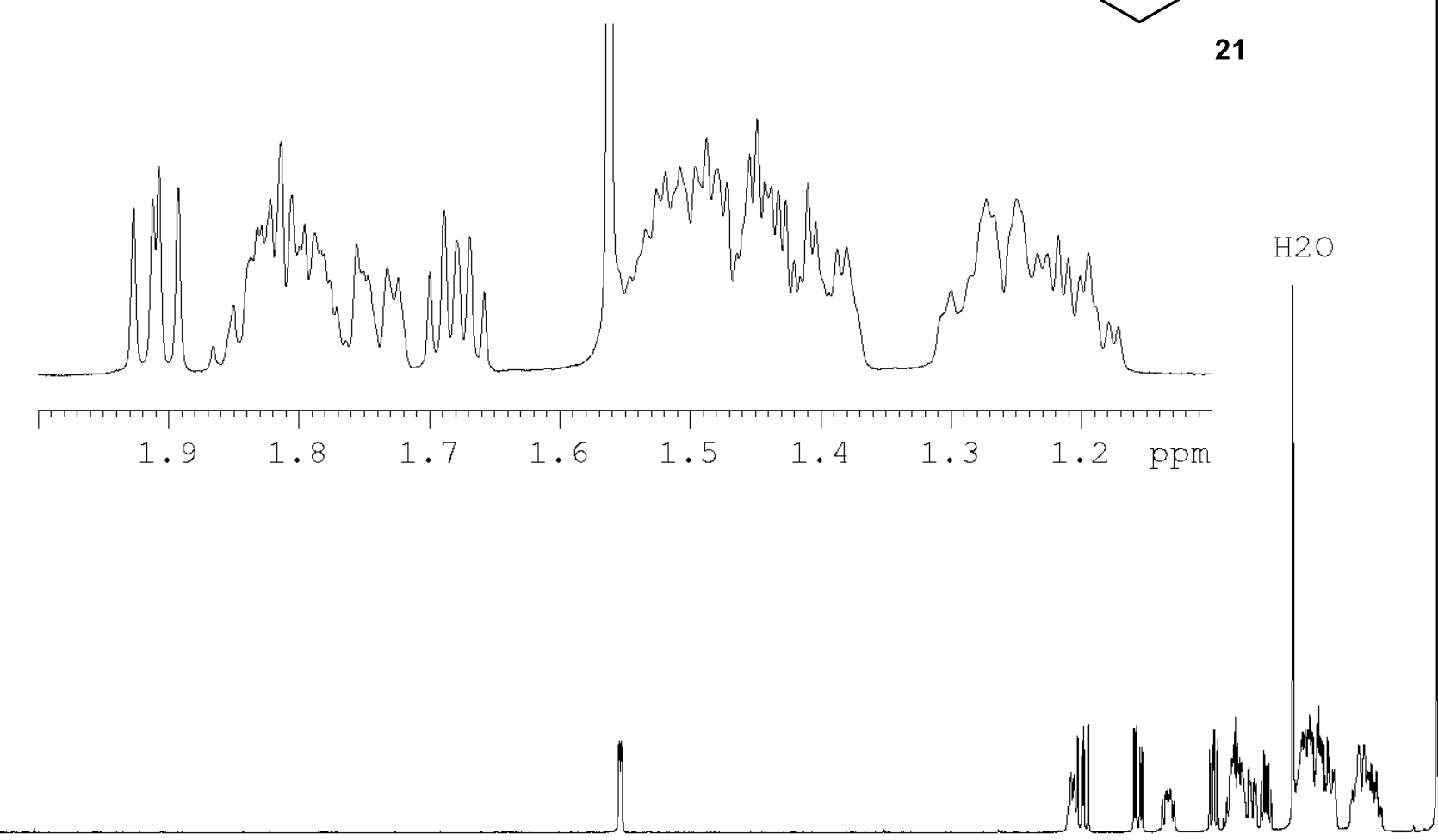

$\begin{array}{llllll}7.5 & 7.0 & 6.5 & 6.0 & 5.5 & 5.0\end{array}$

$\left|\begin{array}{c}\infty \\ 0 \\ 0 \\ 0\end{array}\right|$

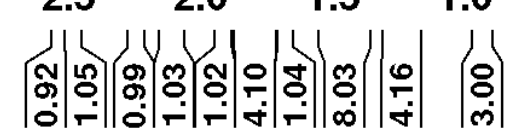

ppm 

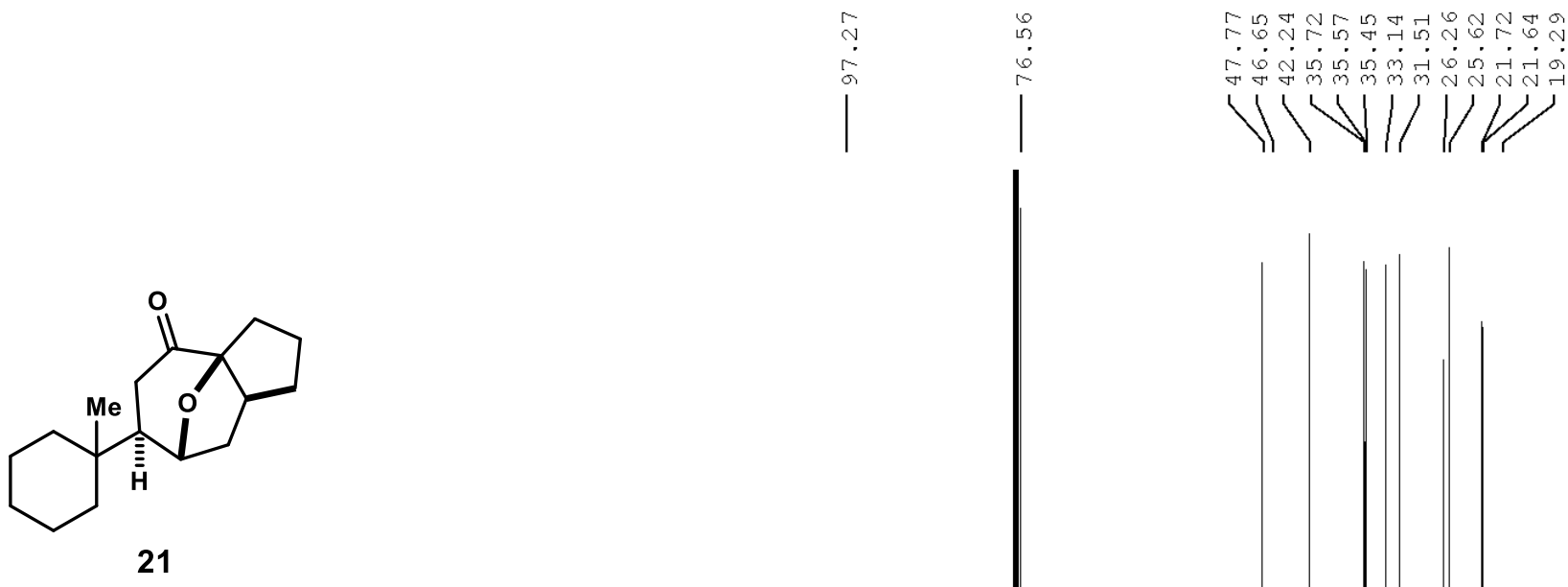

Current Data Parameters

PROCNO

$2-299$
4

F2 - Acquisition Parame

Date_ 20200208

INSTRUM

PROBHD

PULPROG

SOLVENT

NS

NS

SWH

SWDH

FID
AQ
RG
DW

$\mathrm{DE}$

TE

D11

av600

$\begin{array}{lr}====== & \text { CHANNEL } \mathrm{f1}===: \\ \text { SFO1 } & 150.9194080 \\ \text { NUC1 } & 13 \mathrm{C} \\ \text { P1 } & 10.10 \\ \text { PLW1 } & 64.00000000 \\ & \\ ======= & \text { CHANNEL } f 2===: \\ \text { SFO2 } & 600.1330010 \\ \text { NUC2 } & 1 \mathrm{H} \\ \text { CPDPRG } 2 & \text { waltz } \\ \text { PCPD2 } & 80.00 \\ \text { PLW2 } & 30.0000000 \mathrm{C} \\ \text { PLW12 } & 0.37154001\end{array}$

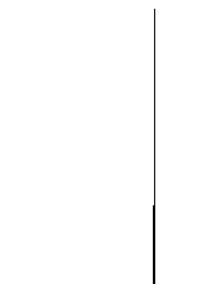

F2 - Processing paramet

$\begin{array}{lr}\text { SI } & 65536 \\ \text { SF } & 150.9028085\end{array}$

WDW EM

$\begin{array}{lll}S S B & 0 & 1.00\end{array}$

$\begin{array}{lll}\mathrm{GB} & 0 & 1.00\end{array}$

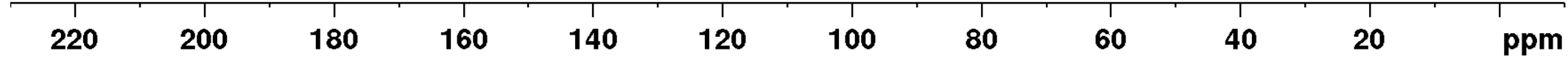


E2 - Rcquisition Parane
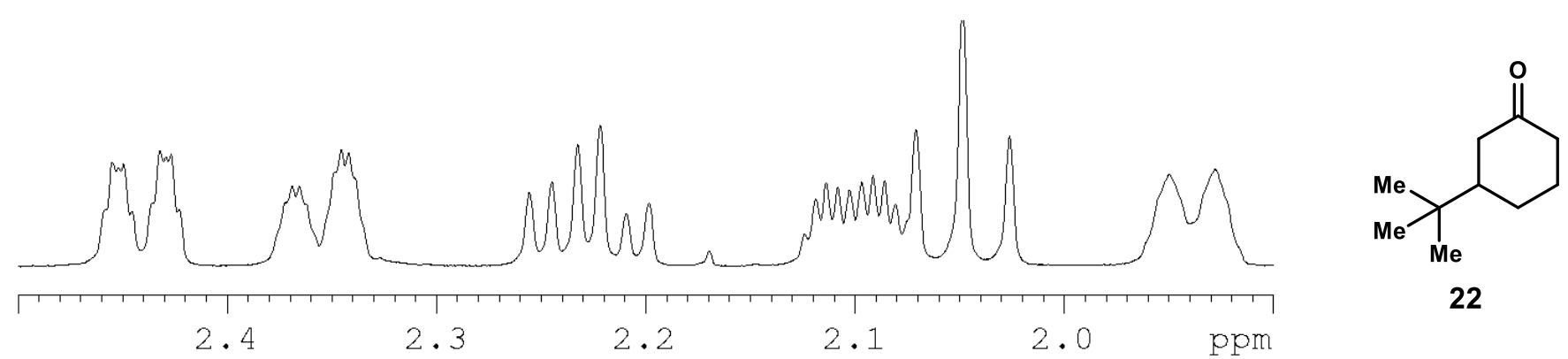

Date

20190514

Time

av600

PROBHD $5 \mathrm{~mm}$ CPBBO BB-

PULPROG $\quad$ zg30

TD 98074

SOLVENT

DS

SWH

FIDRE

RG

DW

$\mathrm{DE}$

TE

TDO

$===$

$\mathrm{SFO1}$

P1

P1
PLW1

2
2
9615.385 0.098042
5.0998478

18
52.000

13.81

0.10000000

F2 - Processing paramet

SI $\quad 65536$

WDW

$S S B$

G

1.60

1.55

1.45

1.40

1.35

ppm

$\mathrm{H} 2 \mathrm{O}$

Mandind Whathe

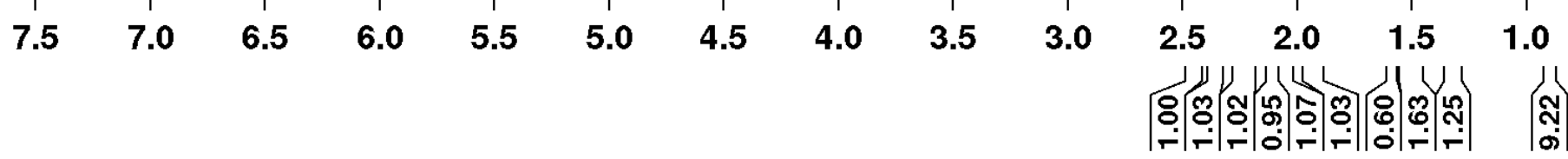


<smiles>CC(C)(C)[C@H]1CCCC(=O)C1</smiles>

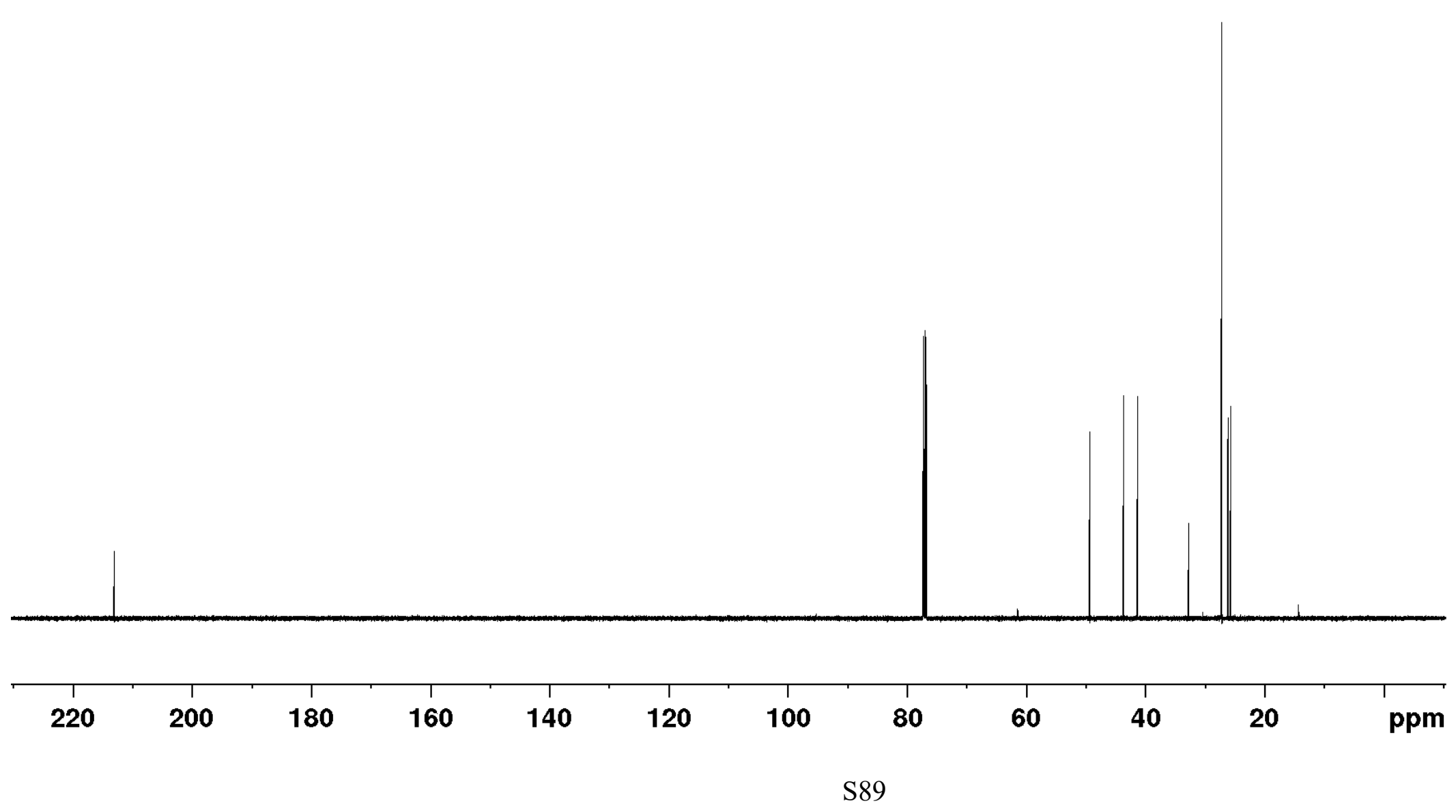

Current Data Parameters

EXPNO

PROCNO

F2 - Acquisition Parame Date

Time

INSTRUM

TD ROG SpInEchopg 30 gk

SOLVENT

NS

DS

FIDRES $\quad 0.462388$

$\mathrm{AQ}$

RG

$\mathrm{DE}$

TE

D1

d11
D16

d17

MCREST

MCWF

2

1.0813440

7298.2

6.00
298.0

1.20000005

0.03000000

$0.0002000 \mathrm{C}$

0.00019600

$=====$
NUC1

P1

P12
P20

PLO

PLI

SP2

SP 4

Crp60comp.4 SPNAM [4] Crp60, 0.5,20.1 SPOFF2 $0 \mathrm{~Hz}$

$======$ CHANNEL $£ 2===$ CPDPRG [2 waltz16

NUC2

PCPD2

$\mathrm{PL12}$

100.00

1.60

500.2225011 


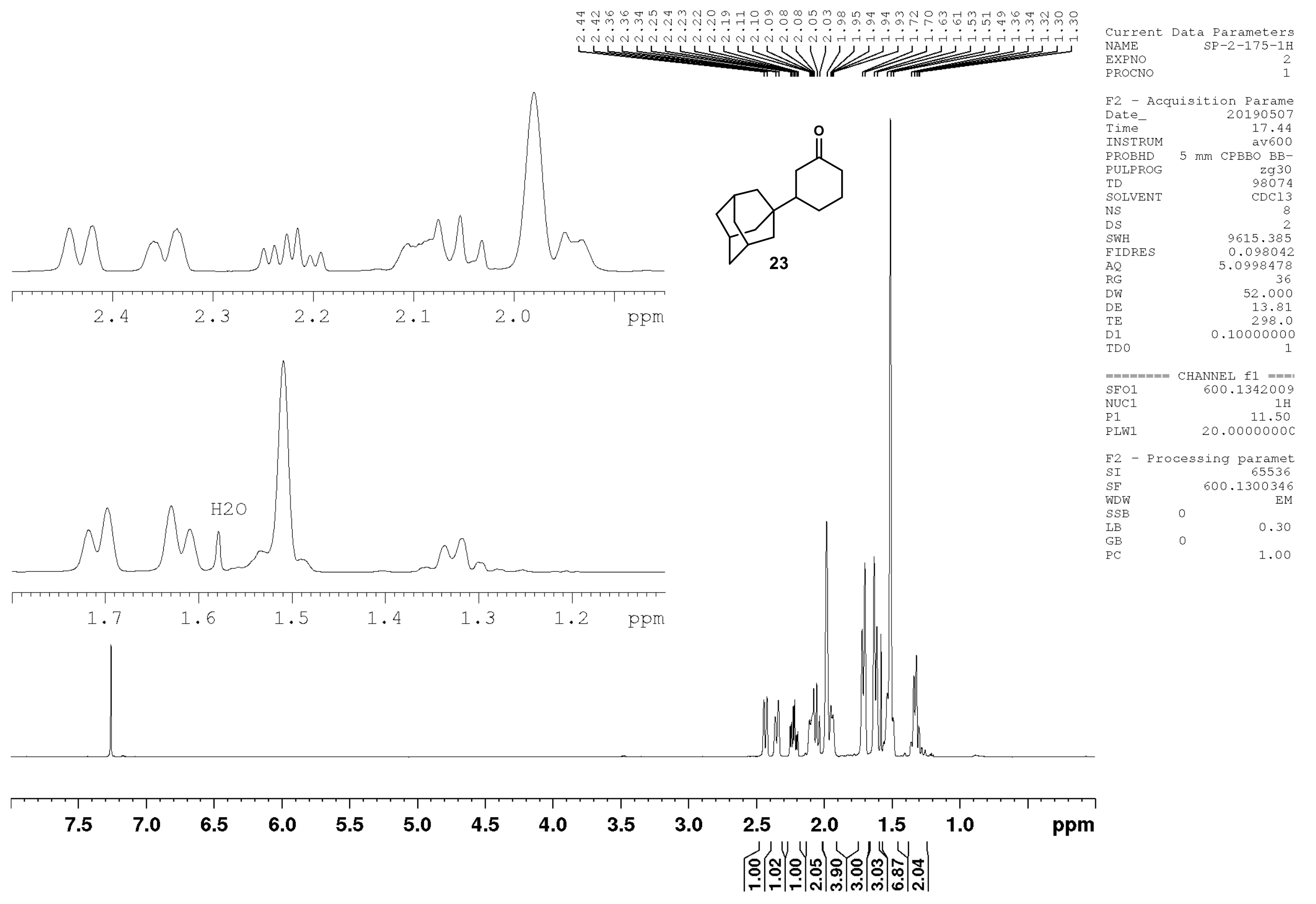




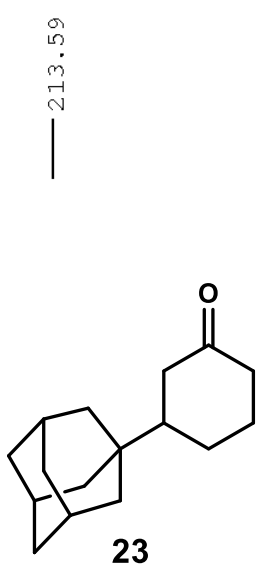

PROCNO

F2 - A

Daterion Parame Dime-

TINSTRUM

IROBHD

PUT PROG

$\begin{array}{r}17.40 \\ \hline\end{array}$

TD

NS

DS

FIDRES

$\mathrm{AQ}$
$\mathrm{RG}$

RG

DE

TE

D1
D11

D11
TD0

$====$

SFO1

NUC1
P1

P1
PLW1

zgda30
65536

$\mathrm{CDCl}$
217

36231.883

0.552855 0.9043968 2050
13.800

18.01
298.0

0.40000001 0.03000000

$=$ CHANNEL $\mathrm{f} 1===$

150.9194080
$13 \mathrm{C}$

$=======$ CHANNEL $\mathrm{f} 2===$

SFO2 600.1330010

NUC2 $1 \mathrm{H}$

CPDPRG 2 waltz16

PLW2

20.00000000

F2 - Processing paramet

SI

SF

WDW

150.9028085

1.00

$\mathrm{PC}$

1.00

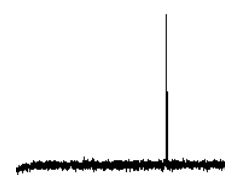

\begin{tabular}{|rrrrrrrrrrrr}
\hline 220 & 200 & 180 & 160 & 140 & 120 & 100 & 80 & 60 & 40 & 20 & ppm
\end{tabular}




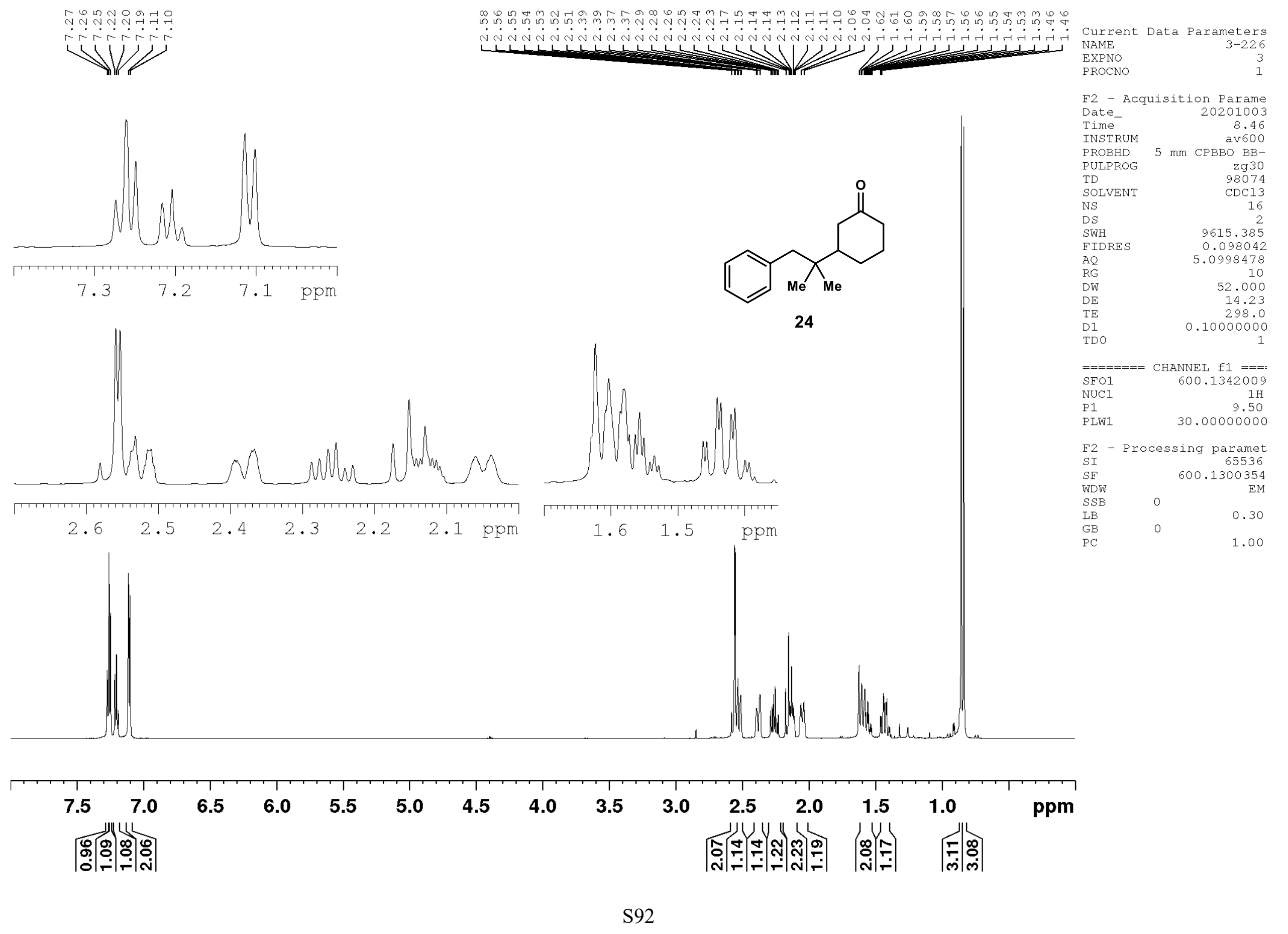


|⿱⺈⿻コ一心

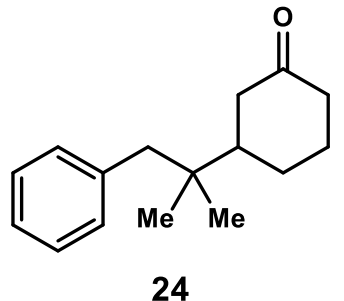

24

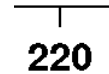

200

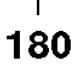

180

160

140

120

100

80

60

40

20

ppm
Current Data Parameters

EXPNO

F2 - Acquisition Parame Date

Date_

INTe

PROBHD

TD

SOLV

DS

AQ

RG

$\mathrm{DE}$

TE

D1
D11

TDO

$=====$

$\mathrm{SFO1}$

NUC1
P1
PLW1

0.9043968

2050
13.800

19.63

1.20000005

0.03000000

CHANNET 91

150.9194080

$13 \mathrm{C}$
10.10

64.00000000

$=======$ CHANNEL $f 2===$

$\mathrm{SFO} 2$

NUC2

CPDPRG

PCPD2

600.1330010

waltz16

$30.0000000 \mathrm{C}$

PLW12

0.37154001

F2 - Processing paramet

SI

$\begin{array}{ll}\text { SF } & 150.9028085\end{array}$

WDW

SSB

LB

$G B$
$P C$

EM

1.00

1.00 
F2 - Acquisition Parame
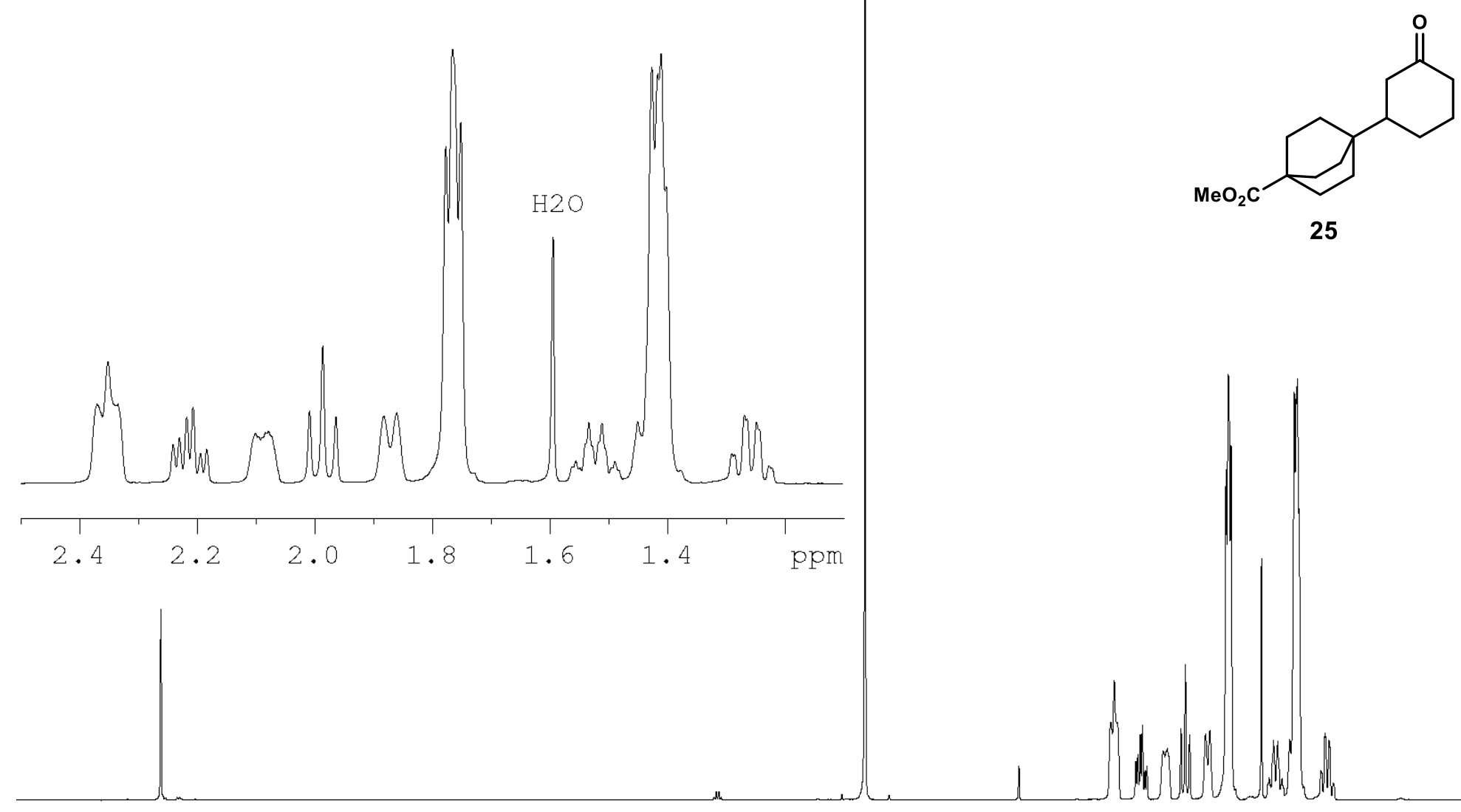

NS

DS

$\begin{array}{lr}\text { DS } & 2 \\ \text { SWH } & 9615.385 \\ \text { FIDRES } & 0.098042\end{array}$

$A Q$

RG

DW

DE

D1

25 5.0998478

TDO

52.000

13.81

0.10000000

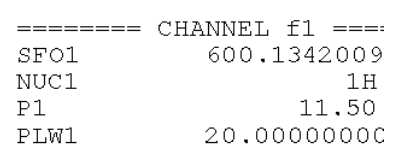

PLW1

$20.0000000 \mathrm{C}$

F2 - Processing paramet

SI 65536

$\begin{array}{ll}\mathrm{SF} & 600.1300348\end{array}$

WDW

EM

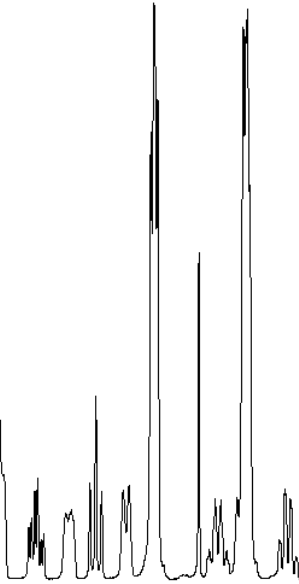

0.30

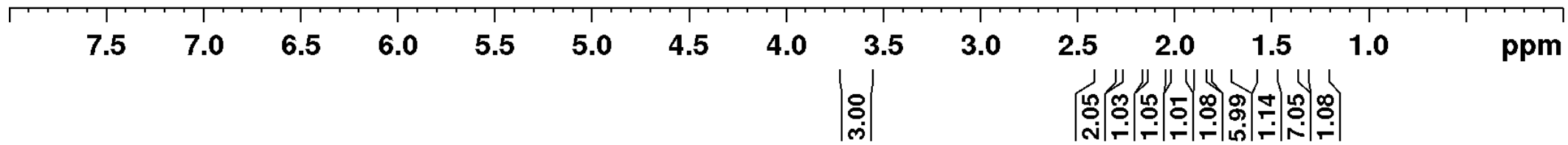



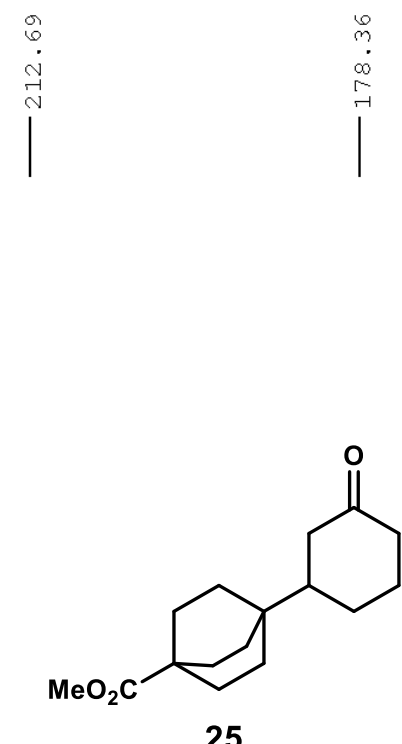

25
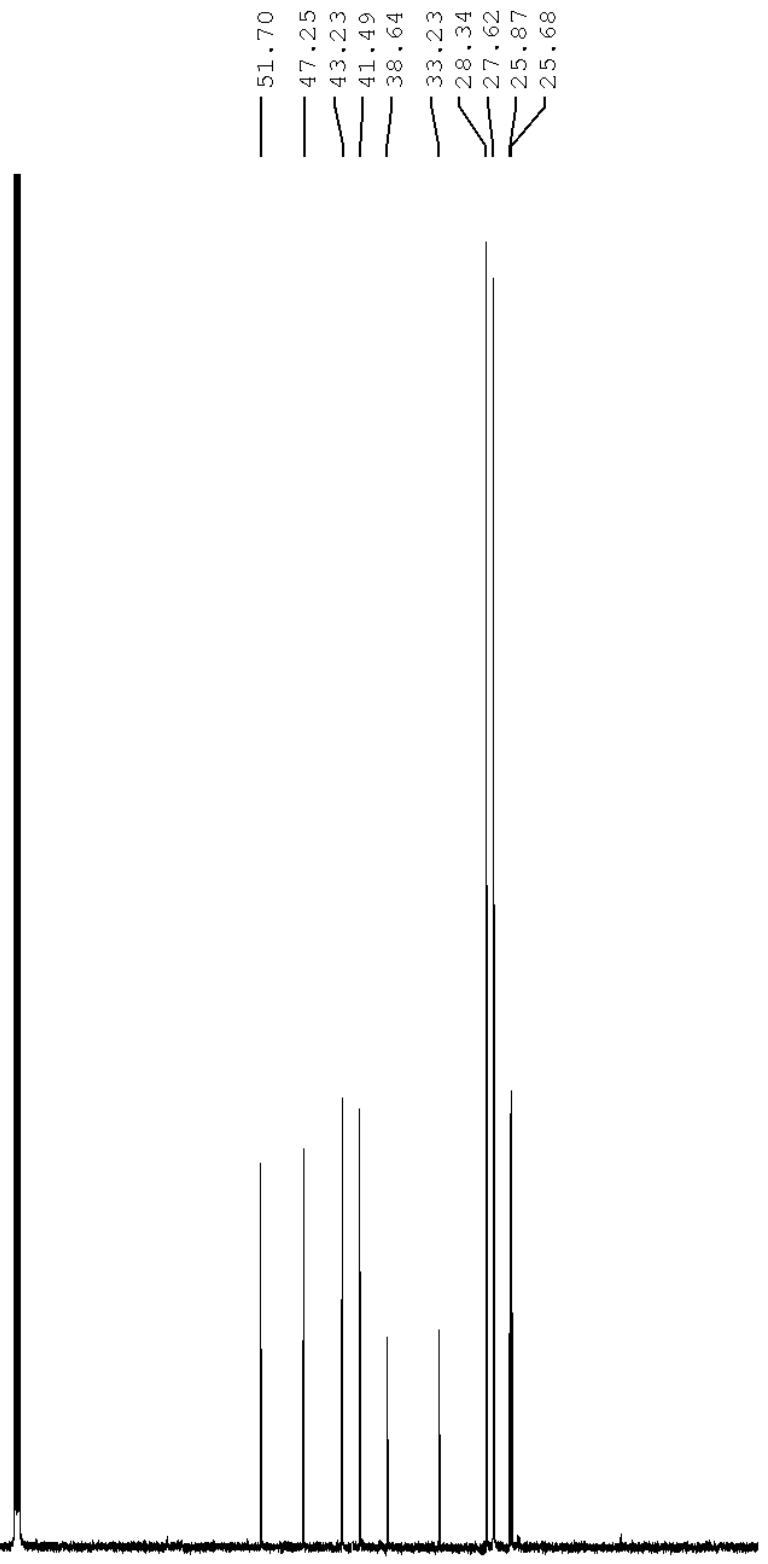

PLW1

CPDPRG [2

PCPD 2

PLW2 2

$\mathrm{HB}$

GB
PC
FO1

UC1

$===$

$\mathrm{FF} 2$

0.32734001

2 - Processing paramet 65536
150

SB

ta Parameters

- Acquisition Parame 20190508 av 600 $5 \mathrm{~mm}$ CPBBO BBzgde 30 0
36231.883 36231.883
0.552855 0.9043968 13.800 18.01
298.0 0.40000001 0.03000000 CHANNTI $f 1==$ 150.9194080 64.00000000 HANNEL $12===$ 600.1330010 $1 \mathrm{H}$
walt $z 16$ 0.00000000 150.9028085 0

1.00 1.00

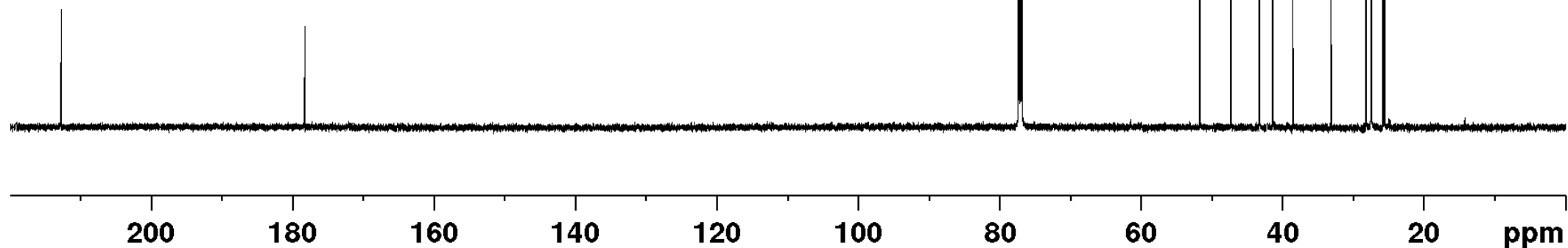




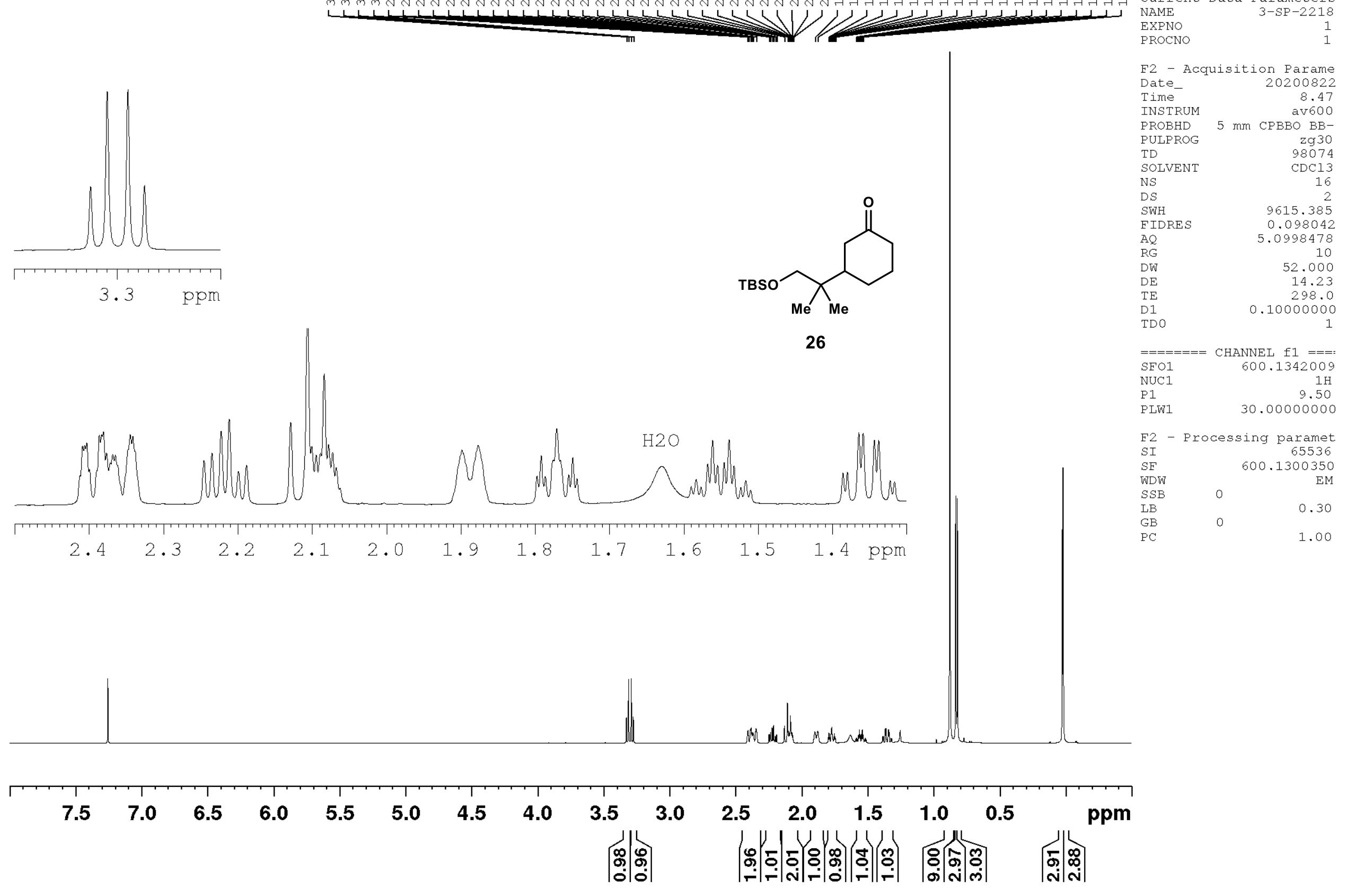


26

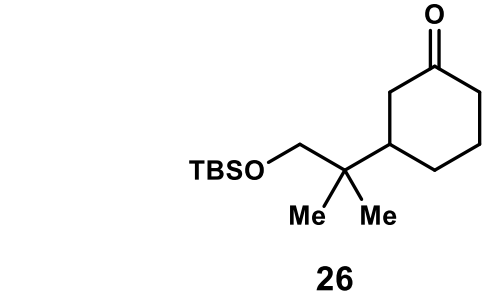

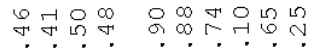

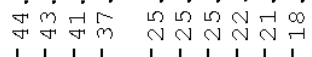

V/I V VI
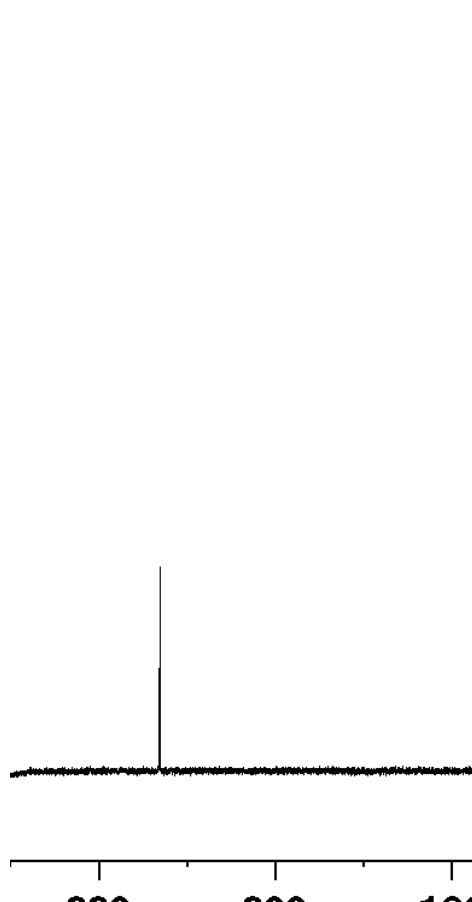

220

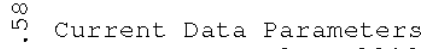
एि

$\checkmark$ EXPNO

F2 Date Acquisition Parame Date

Time

INRTRD

PUT $R$ ROG

9.13

PULPRO

TD
SOLVENT

NS

$\begin{array}{lr}\text { DS } & 4 \\ \text { SWH } & 36231.883\end{array}$

AQ $\quad 0.9043968$

$\mathrm{DW}$

TE

D1

D11
TD0

$=====$

$\mathrm{SFO1}$

NuC

P1

mm $\mathrm{CPBBO}$ BBzgdc 30

PLW1

$===$

$\mathrm{SFO} 2$

CPDPRG [2

PCPD2

PLW2 2

PLW12

F2 - Processing paramet SI

WDW

WDW

LB

GB
PC

\section{1}

150.9194080

10.10

64.00000000

HANNEL $£ 2===$

$1 \mathrm{H}$
$\mathrm{wal}$
$\mathrm{z} 16$

80.00

30.00000000

sing paramet

150.9028085

0

1.00

1.00 

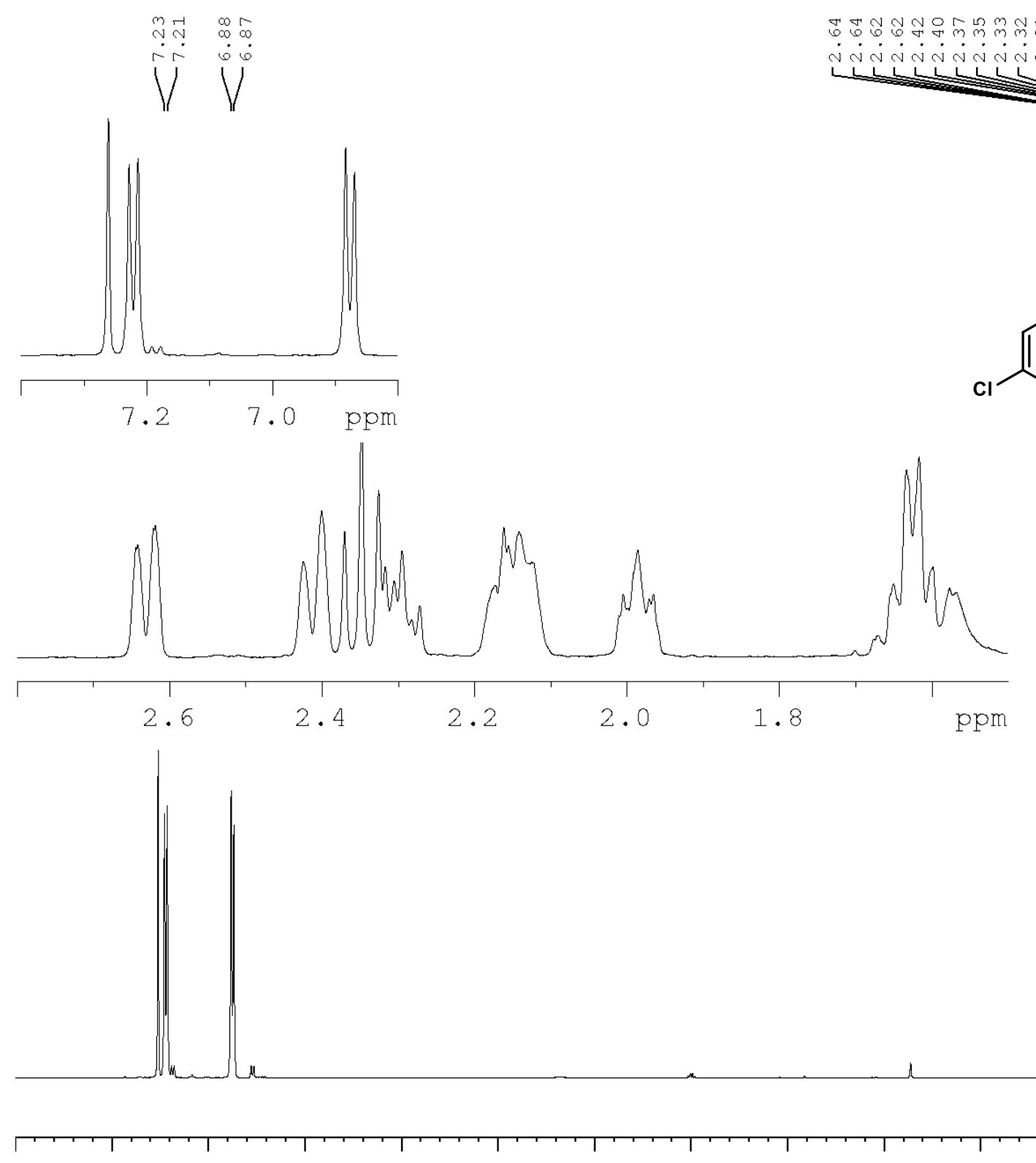

$\begin{array}{lr}\text { Current } & \text { Data Parameters } \\ \text { NAME } & \text { SP-2-235-1H } \\ \text { EXPNO } & 1 \\ \text { PROCNO } & 1\end{array}$

F2 - Acquisition Parame Date_r 20190618 $\begin{array}{lr}\text { Time } & 17.35 \\ \text { INSTRUM } & \text { av600 }\end{array}$ PROBHD $5 \mathrm{~mm}$ CPBBO BBPULPROG
zg 30 TD $\quad 98074$ SOLVENT CDC13 DS

DS

SWH $\quad 9615.385$ AQ $\quad 0.098042$ $\begin{array}{lr}\text { AQ } & 5.0998478 \\ \text { RG } & 45.2\end{array}$ DW DE TE
D1 TDO

$=======$ CHANNEL $\mathrm{fl}===:$
SFO1 $\begin{array}{lr}\text { SFO1 } & 600.1342009 \\ \text { NUC1 } & 1 \mathrm{H}\end{array}$ $\begin{array}{lr}\text { NUC1 } & 1 \mathrm{H} \\ \mathrm{P} 1 & 12.00\end{array}$ $\begin{array}{ll}\text { P1 } & 30.00000000\end{array}$ F2 - Processing paramet SI $\quad 65536$ SWW $\quad 600.1300344$ SSB 0 LB GB

1.00

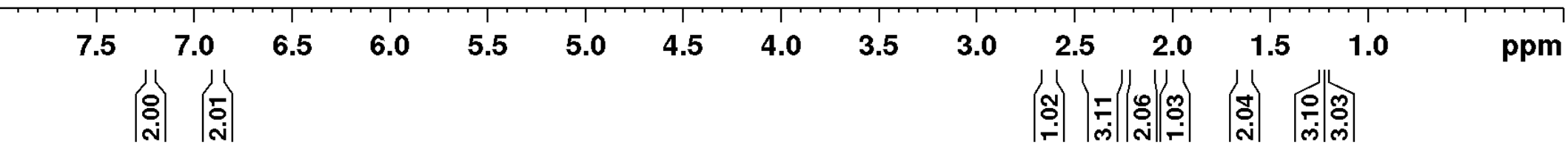


<smiles>CC(C)(Oc1ccc(Cl)cc1)C1CCCC(=O)C1</smiles>

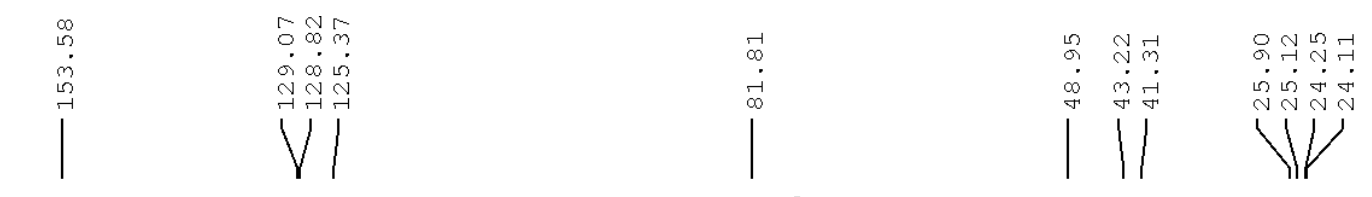

PROCNO

F2 - Acquisition Parame Date

Time-

INSTRUM

TD

SOLVEN

NS

DS

$\begin{array}{lr}\text { DS } & 0 \\ \text { SWH } & 36231.883 \\ \text { FIDRES } & 0.552855\end{array}$

$\mathrm{AQ}$

RW

DE

TE

D1
D11
TD0

\section{$====$}

SFO1
NUC1

NUC1
P1

0.9043968

2050
13.800

19.63
298.0

0.40000001

0.03000000

PLW1

CHANNEL $\mathrm{f1}===$

150.9194080
$13 \mathrm{C}$

64.00000000

$=======\quad \begin{gathered}\text { CHANNEL } f 2== \\ \text { SFO2 }\end{gathered} \quad$ 600.1330010

$\mathrm{SFO} 2$

600.1330010
$1 \mathrm{H}$
waltz 16
80.00

$\begin{array}{lr}\text { CPDPRG [2 } & \text { waltz16 } \\ \text { PCPD2 } & 80.00 \\ \text { PIW2 } & 30.0000000\end{array}$

PLW12

$30.0000000 \mathrm{C}$

F2 - Processing paramet
SI

$\begin{array}{ll}S F & 150.9028085\end{array}$

WDW

EM

1.00

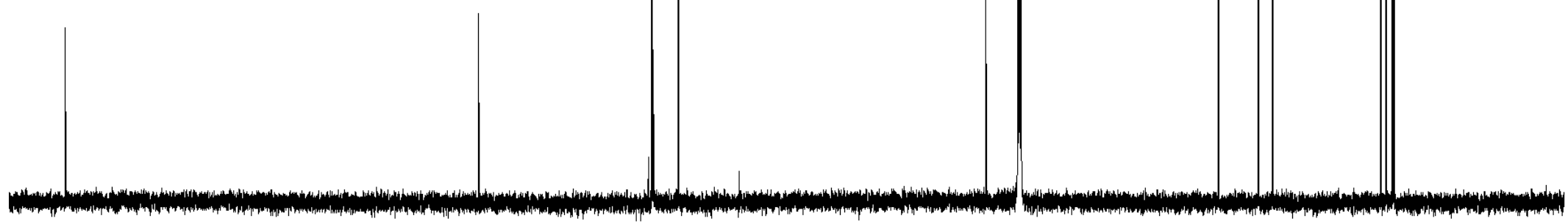

PC 1.00

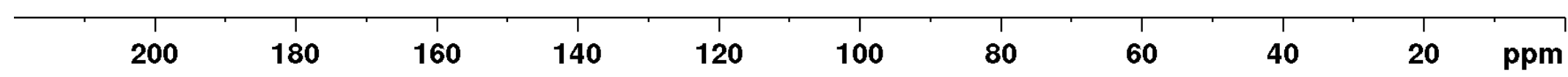




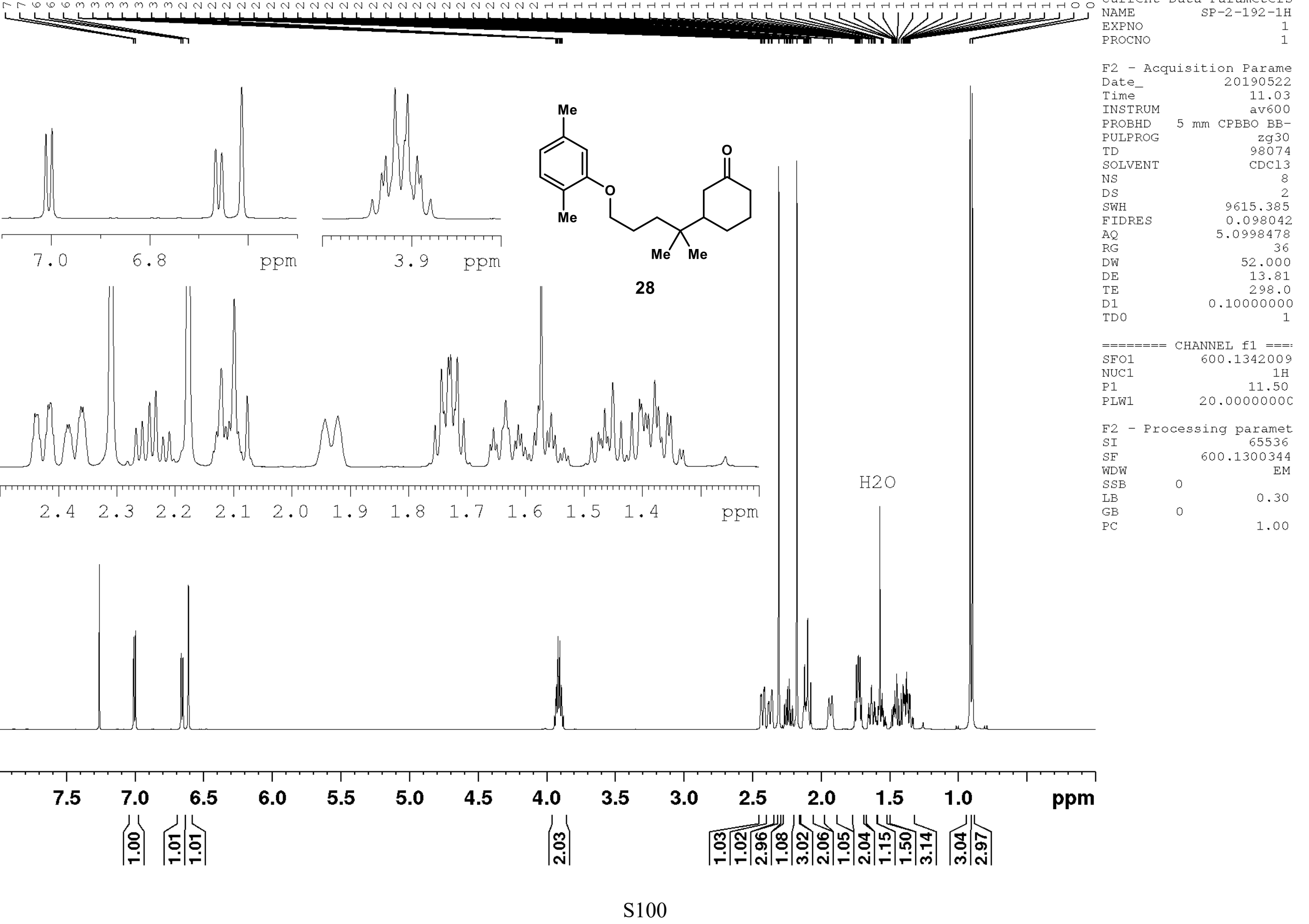


I

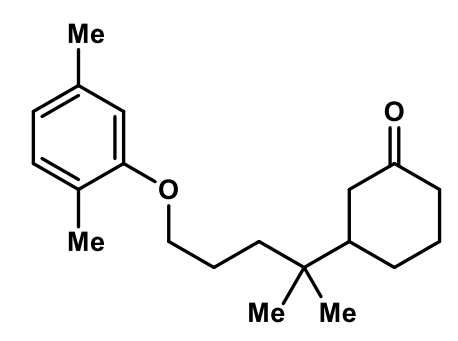

28
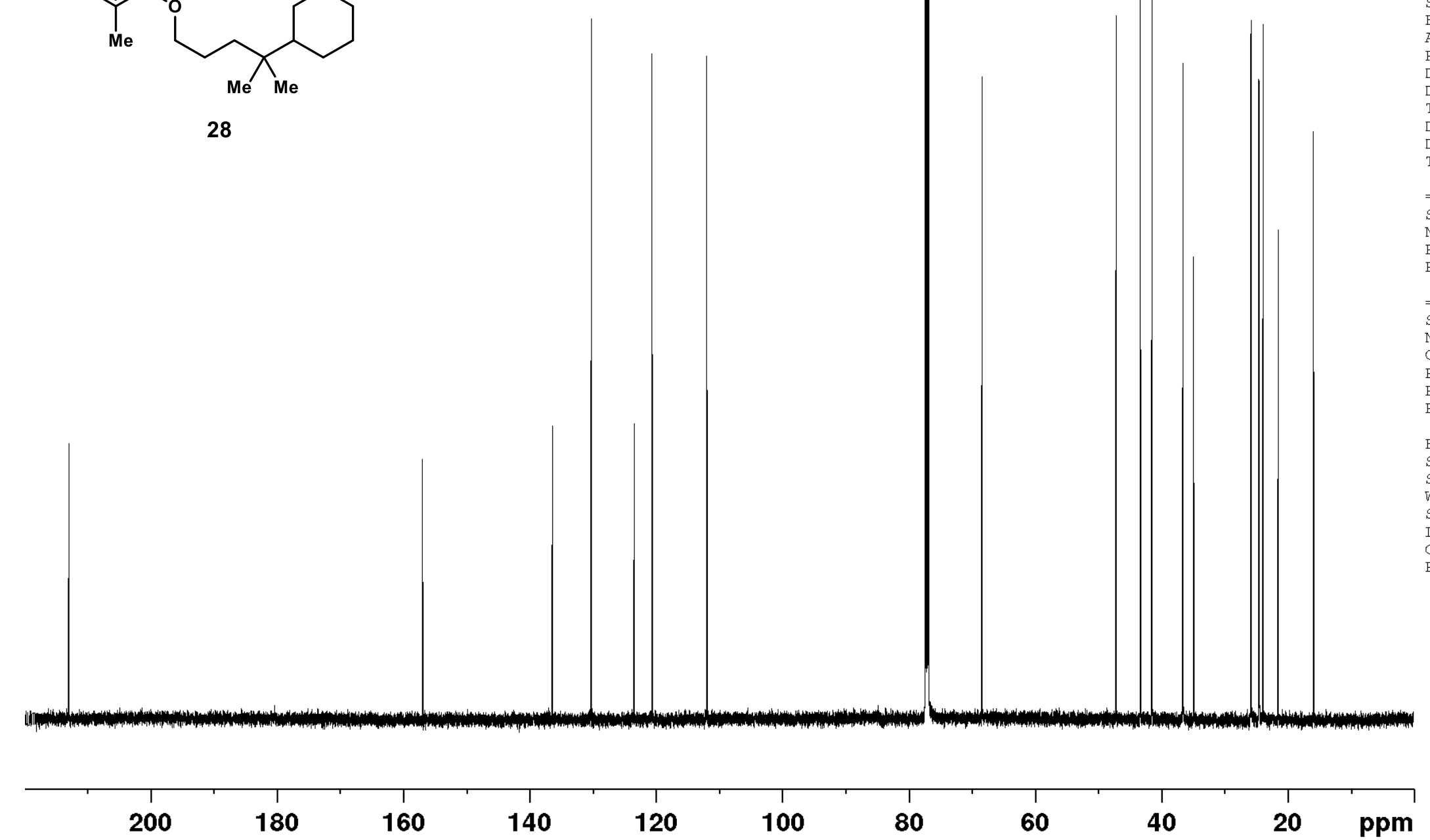

S101

ๆ

Date_

Time

ROBHD $5 \mathrm{~mm}$ CPBBO

TD

SOLVENT

NS

DS

0
36231.883

FIDRES $\quad 0.552855$

AQ

RG
DW

$\mathrm{DE}$

D1

D11

$====$
SFO1
NUC1

PLW1

0.9043968

2050

18.01
298.0

0.40000001 0.03000000

SFO2

NUC2
CPDPRG [

PCPD2

PLW2

1

CHANneL $\mathrm{f} 1===$

150.9194080
$13 \mathrm{C}$

9.60

64.00000000

CHANNEL $12===$

F2 - Processing paramet

SI 65536

$\begin{array}{ll}\text { SF } & 150.9028085\end{array}$

$S S B$

1.00

PC

1.00 


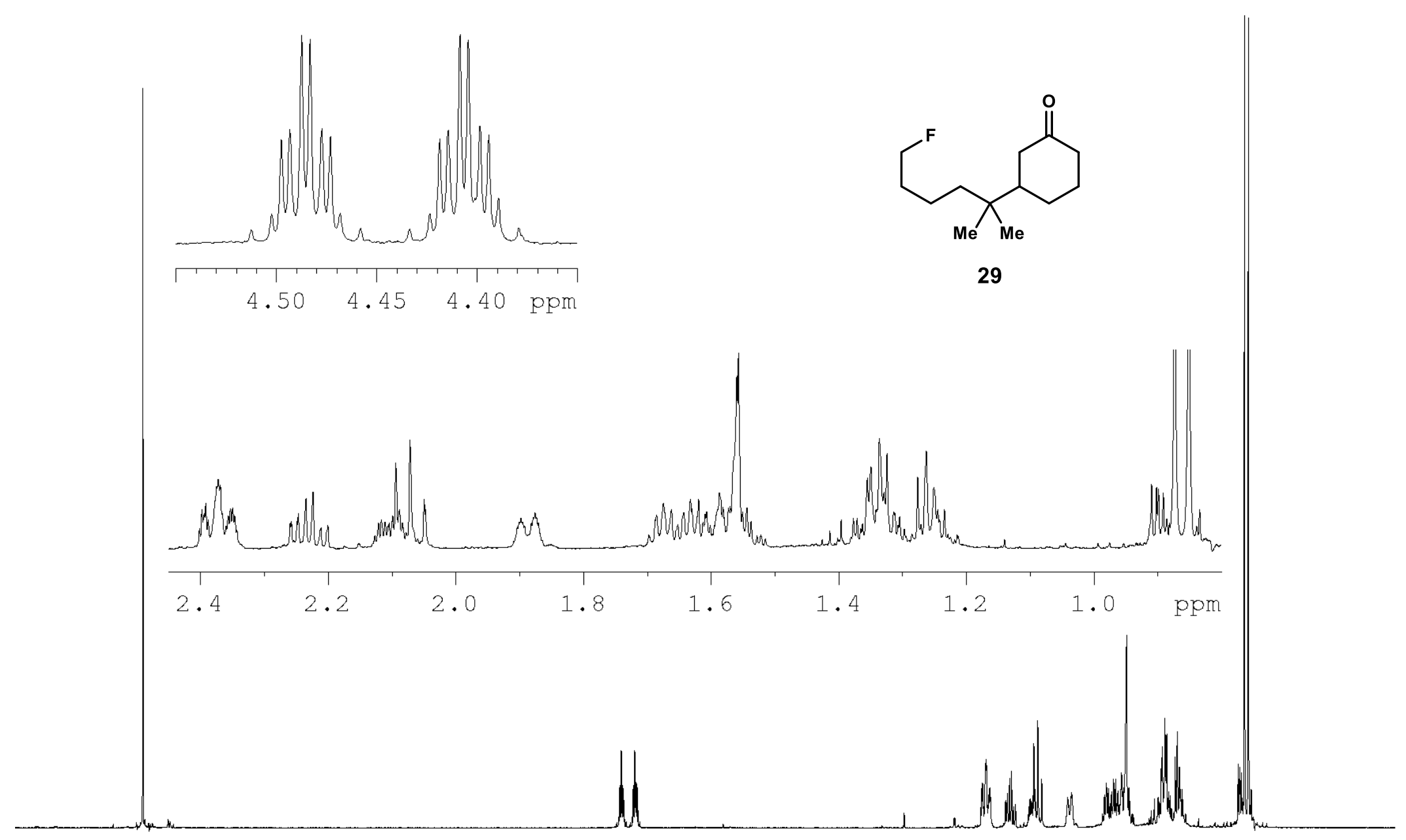

F2 - Acquisition Parame Date_r 20201007 Time 9.03 PROBHD $5 \mathrm{~mm}$ CPBBO BBPULPROG $\quad$ zg 30 $\begin{array}{lr}\text { TD } & \text { Zg30 } \\ \text { TD } & 98074\end{array}$ SOLVENT CDC13

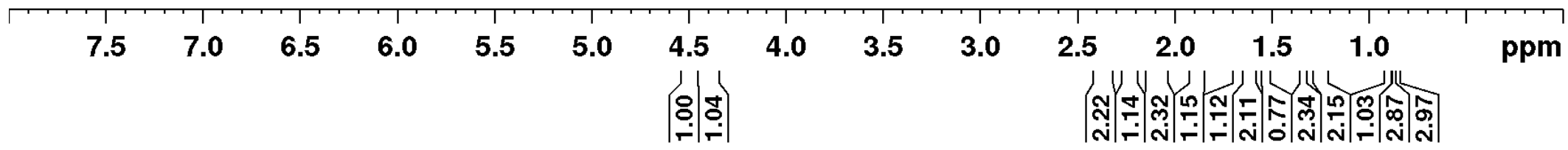




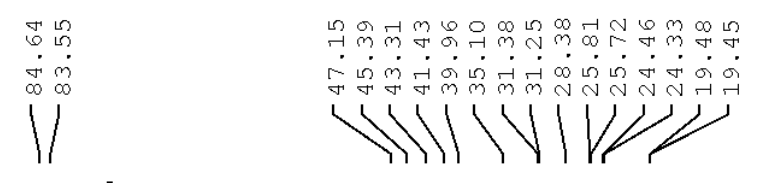

Current Data Parameters

EXPNO

PROCNO

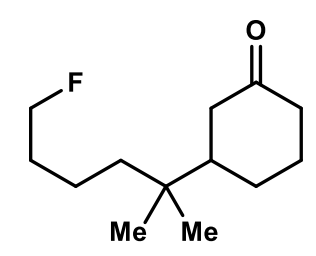

29

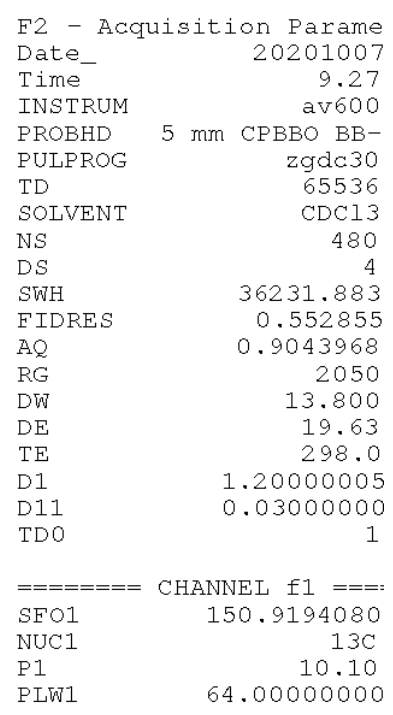

$\mathrm{SFO} 2$

HANNEL $12===$

NUC2 $1 \mathrm{H}$

waltz16

PLW2 30.00000000

PLW12

F2 - Processing paramet

SI 65536

$\begin{array}{ll}\text { SF } & 150.9028085\end{array}$

WDW

SSB

LB

$\mathrm{GB}$
$\mathrm{PC}$

EM

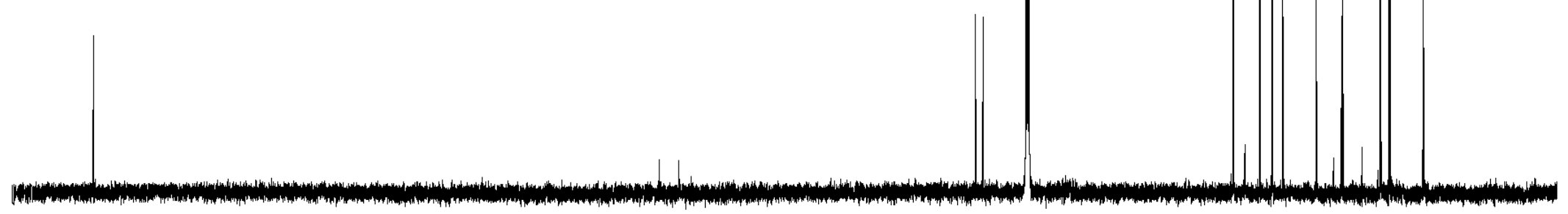

1.00

\begin{tabular}{|c|c|c|c|c|c|c|c|c|c|c|c|}
\hline$T$ & 1 & $T$ & $T$ & $T$ & $T$ & $T$ & $T$ & $T$ & T & 1 & \\
\hline 220 & 200 & 180 & 160 & 140 & 120 & 100 & 80 & 60 & 40 & 20 & ppm \\
\hline
\end{tabular}



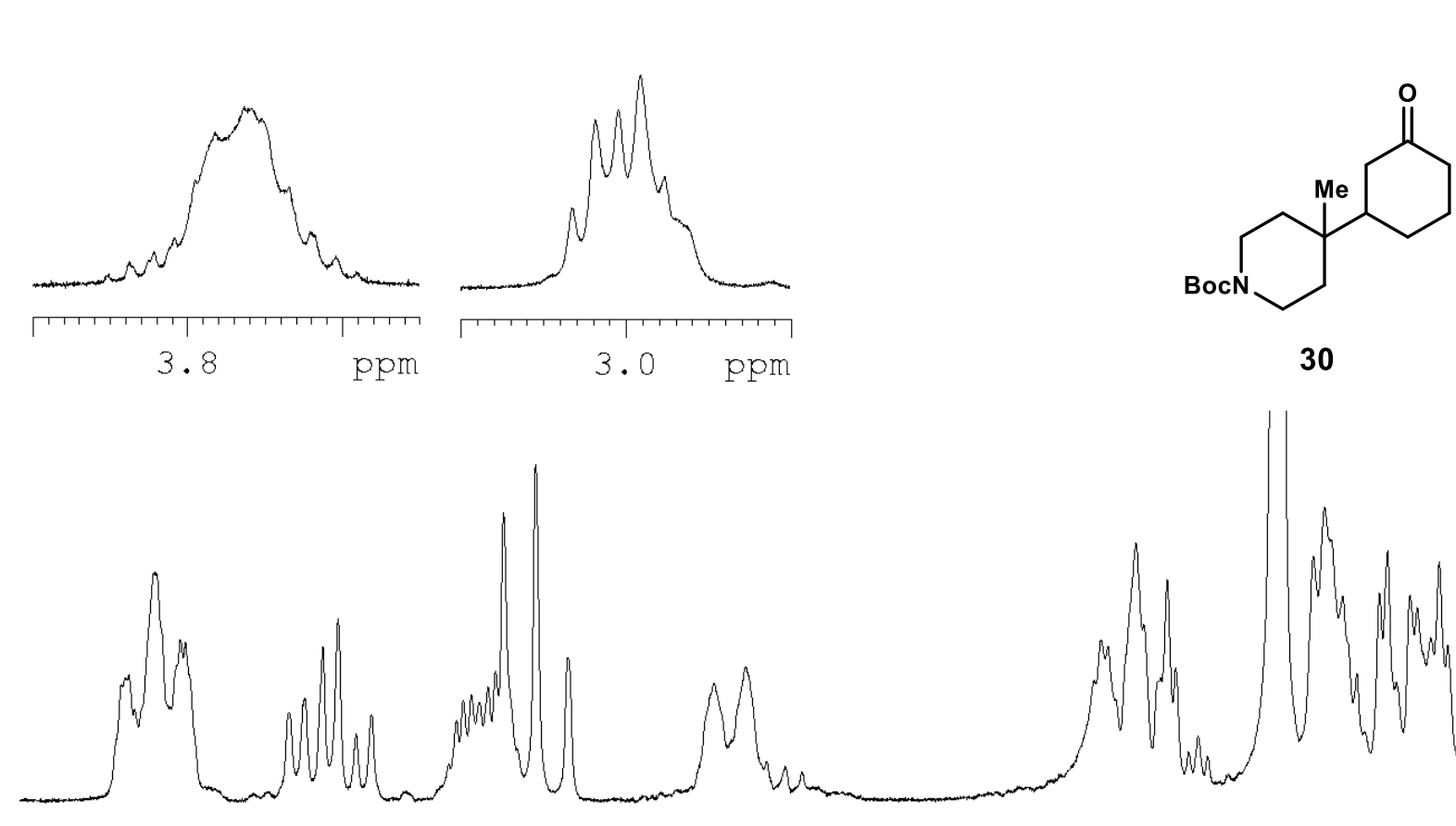

2 .

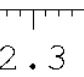

2.2

$2 \cdot 1$

2.01 .9

1.7

$\begin{array}{lll}.5 & 1.4 & 1.3 \mathrm{ppm}\end{array}$ ㅆ․

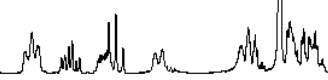

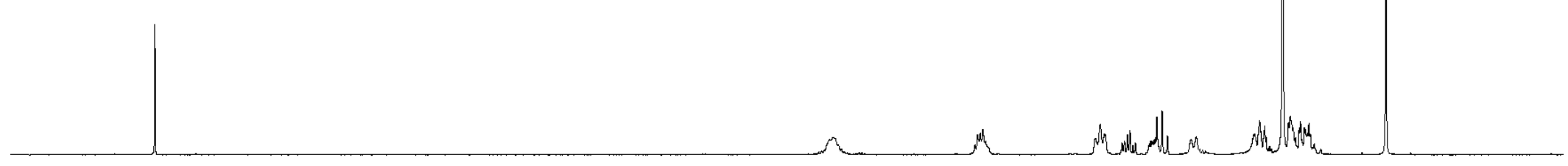

Current Data Parameters

F2 - Acquisition Parane Date Date_

INSTRUM

PROBHD

PUIPROG

TD

NS

DS

FIDRES

$\mathrm{AQ}$
$\mathrm{RG}$

DW

DE

D1

MCREST

MCWRK

$====$
NUC1

P1
PL1

10.06
100500

$5 \mathrm{~mm}$ CPTCI $1 \mathrm{H}$

TCI $1 \mathrm{H}-$

500.2235015

F2 - Processing paramet

SI $\quad 505536$

WDW no

$\begin{array}{lll}S S B & 0 \\ \text { LB } & 0 & H z\end{array}$

GB $\quad 0 \mathrm{~Hz}$

PC

1.00

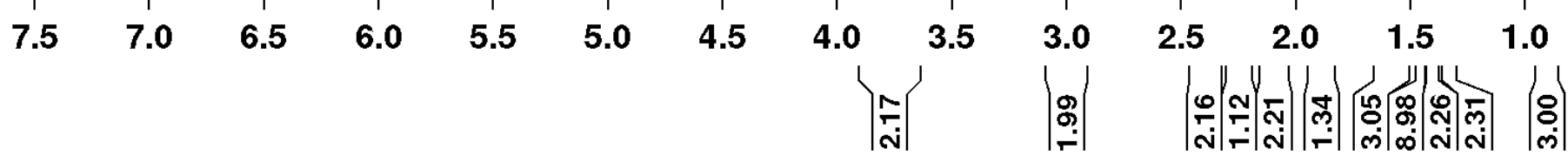




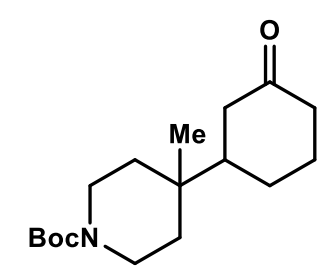

30
Curr

EXPNO

PROCNO

F2 - Acquisition Parame Date

Time-

INSTRUM

10.10
$0 r y 0500$

TD

SOLVENT

NS

$\begin{array}{lr}\text { DS } & 16 \\ \text { SWH } & 30303.031\end{array}$

FIDRES $\quad 0.462388$

$\mathrm{AQ}$

RG

DE

TE

D1 1

D16

d17

MCREST

MCWR

P2

$====$
NuC1

NuC1
P1
P12

P20

PLO

$\mathrm{PL1} 1$
$\mathrm{SFO} 1$

SFO1
SP2
SP 4

SPNAM [2] Crp60comp.4 SPNAM [4] Crp60, 0.5,20.1 SPOFF2 $0 \mathrm{~Hz}$ SPOFF 4 O Hz

$========$ CHANNEL $\mathrm{f} 2===$ CPDPRG [2 waltz16 NUC2

PCPD2

PL2

PL12

$\mathrm{SFO} 2$

1.0813440 7298.2
16.500

6.00
298.0

0.25000000

0.03000000

0.01500000 33.10

CHANNEL $\mathrm{f} 1===$
$13 \mathrm{C}$

2000.00

-100
-00

2.70
2.70

CHAI

100.00

1.60 500.2225011

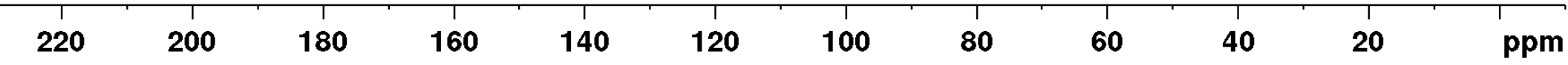




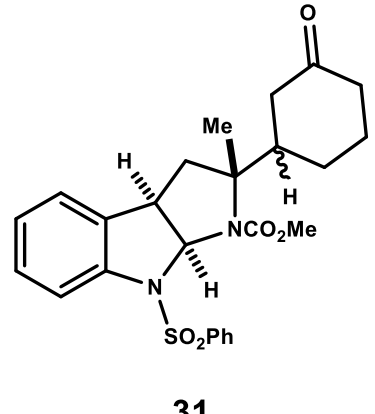

31
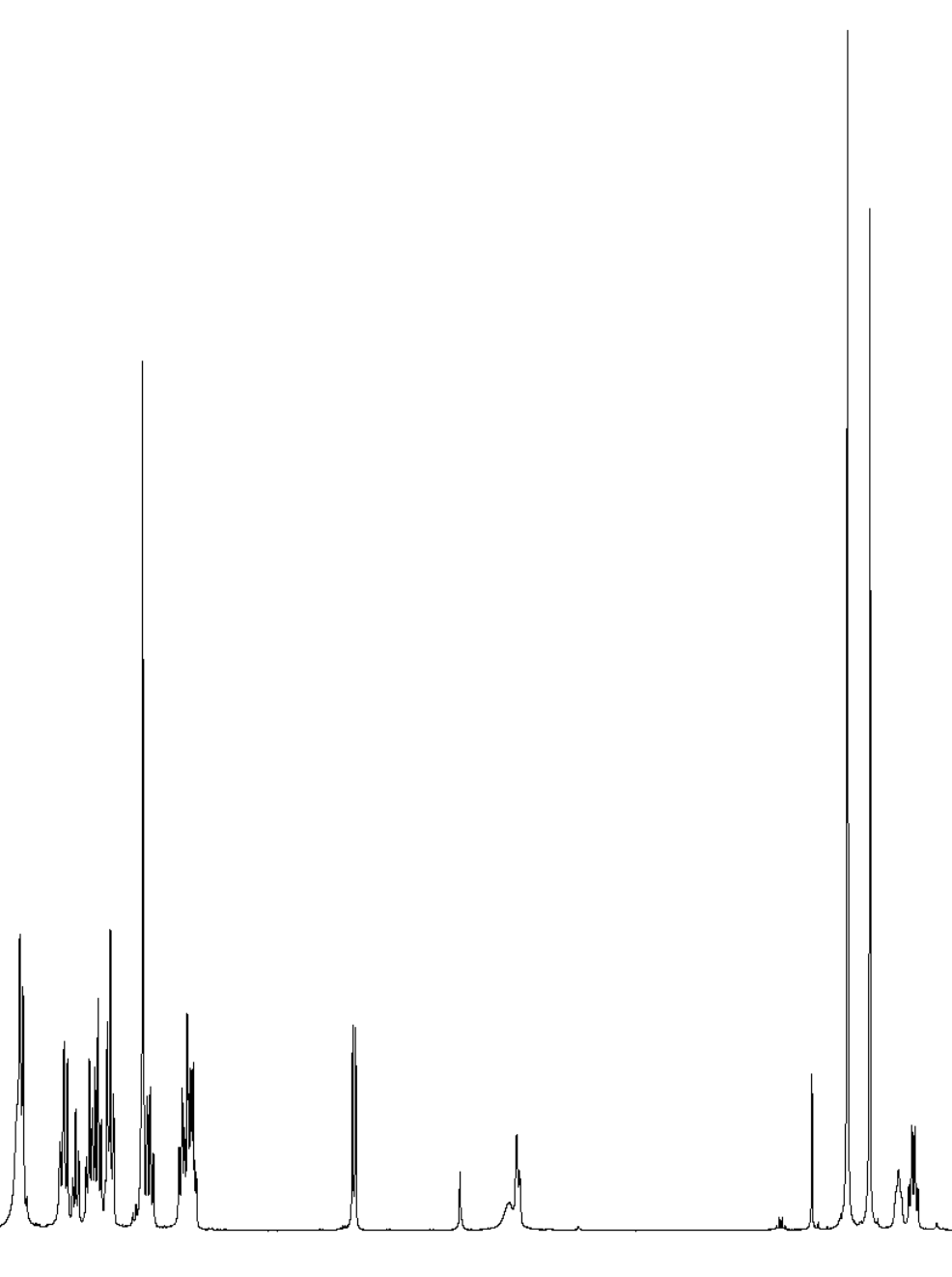

7

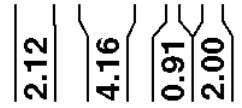

6

$\left|\begin{array}{l}\overline{0} \\ 0\end{array}\right|$
5

$\left|\begin{array}{l}0 \\ 0 \\ 0\end{array}\right|$
4

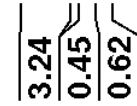

Current Data Parameters NAME EXPNO
PROCNO

F2 - hcquisition Parane on Parame Time

TNSTRUM

PUTPROG

TD

NS

DS

FIDRES

AQ

DW

DE

D1

MCREST

MCWRK

19.51

$5 \mathrm{~mm}$ CPTCT $1 \mathrm{H}$

Cryo500

NuC1

P1

500.2235015

F2 - Processing paramet

SI 65536

$\begin{array}{ll}S F & 500.2200324\end{array}$

SSB 0

LB

GC

1.00 

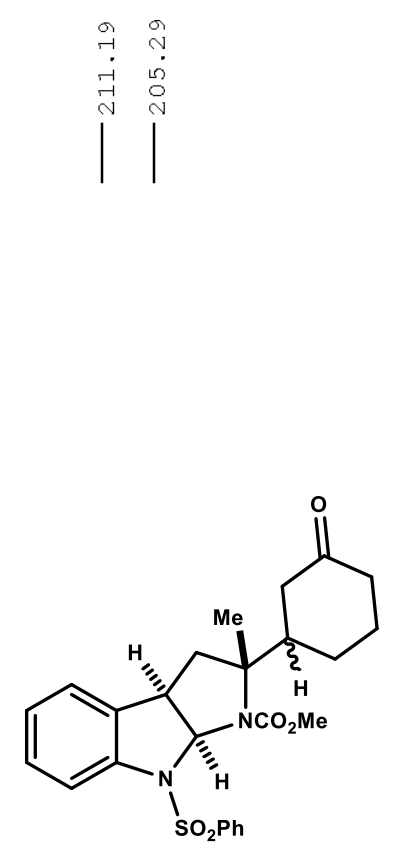

31
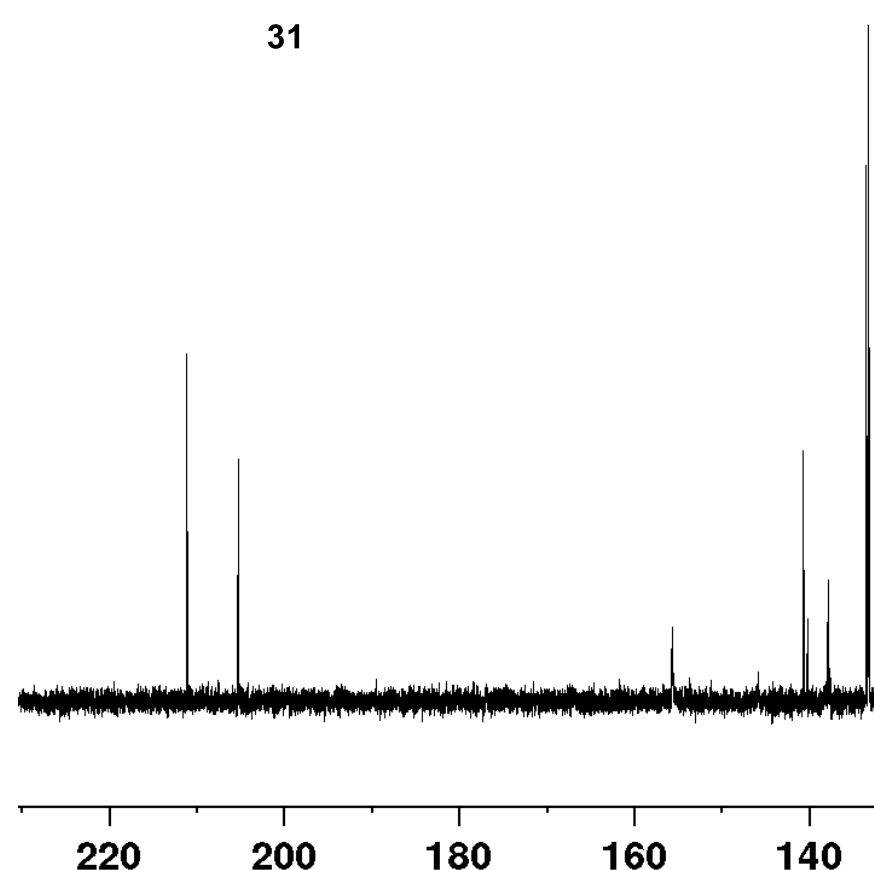

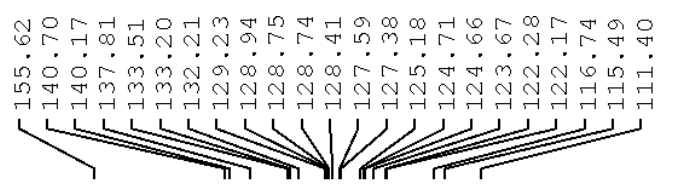

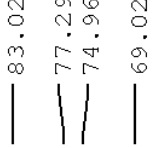
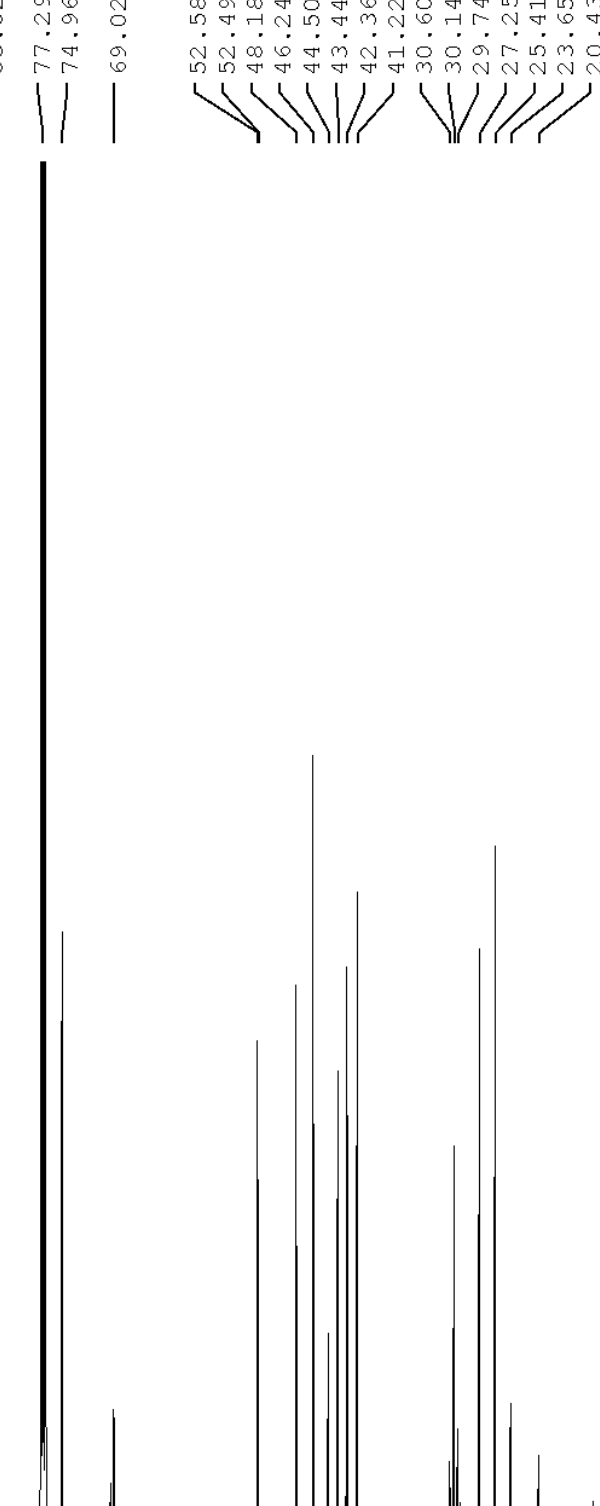

Current Data Parameters NAME

EXPNO
PROCNO

$3-043$
11

F2 - Acquisition Parame

Date__ 20201110

Time

INSTRUM

PROBHD

TD

SOLVENT

NS

DS

SWH $\quad 16$

FIDRES $\quad 0.462388$

$\mathrm{AQ}$

RG

DW

TE

d11

D16
d17

MCREST

MCWRK

P2

1.0813440

4096

16.500

298.0

1.20000005

0.03000000

$0.0002000 \mathrm{C}$

0.00019600

NUC

NUC1

$\begin{array}{lr}\text { P1 } & 13 \mathrm{C} \\ \mathrm{P} 12 & 16.55\end{array}$

PLO $\quad 120.00$

$\begin{array}{ll}P L 1 & -1.00\end{array}$

SP2

125.7942548
2.70
2.70

Crp 60

SPNAM [4] Crp60,0 $5,20.4$

SPOFF2 $0 \mathrm{~Hz}$

SPOFE 4 O

$=======$ CHANNEL f $2===$ :

NUC2

PCPD 2

PL2
PL12

$\mathrm{SFO} 2$

waltz16

100.00
1.60

23.54
500.2225011 

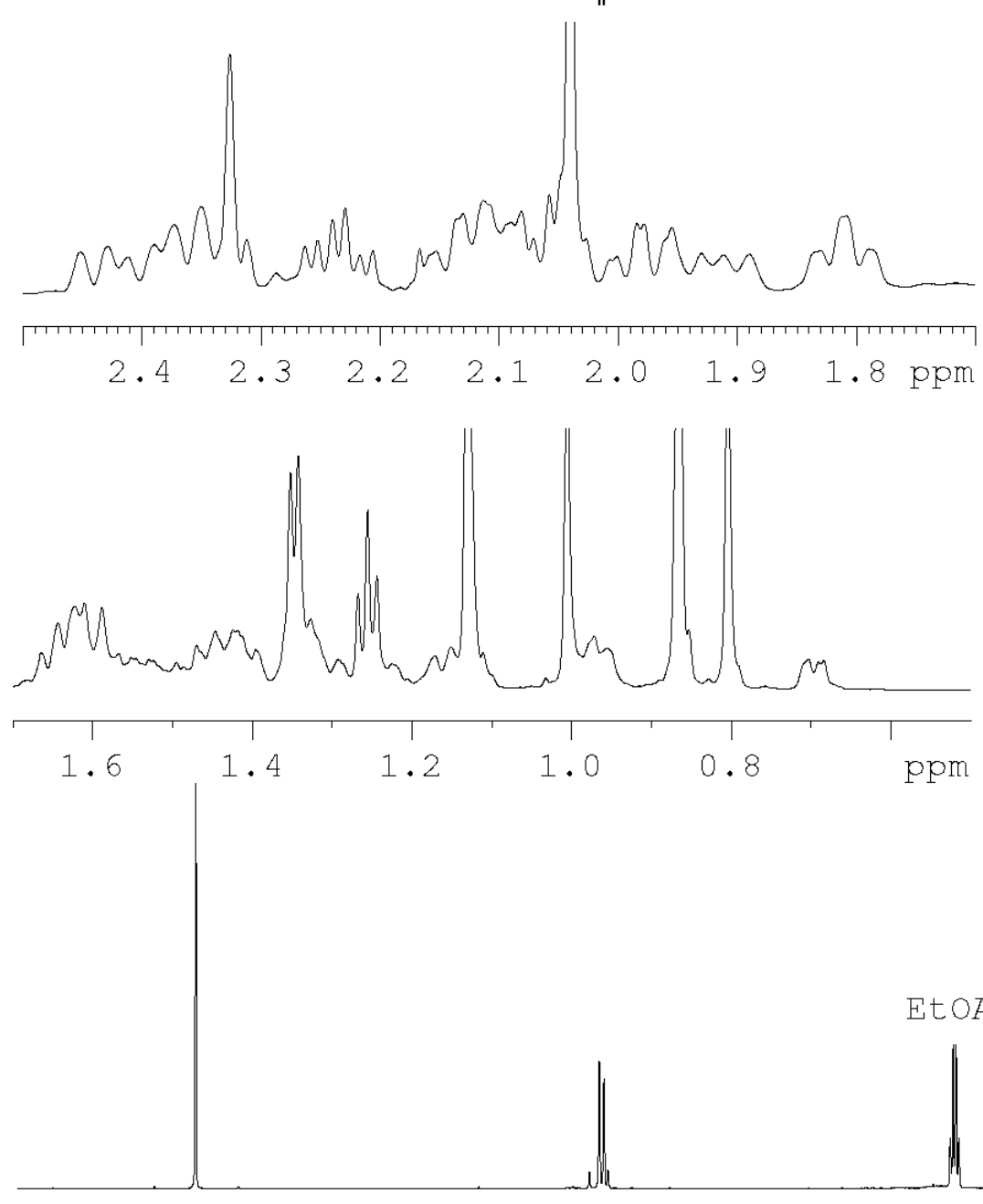

$1.91 .8 \mathrm{ppm}$
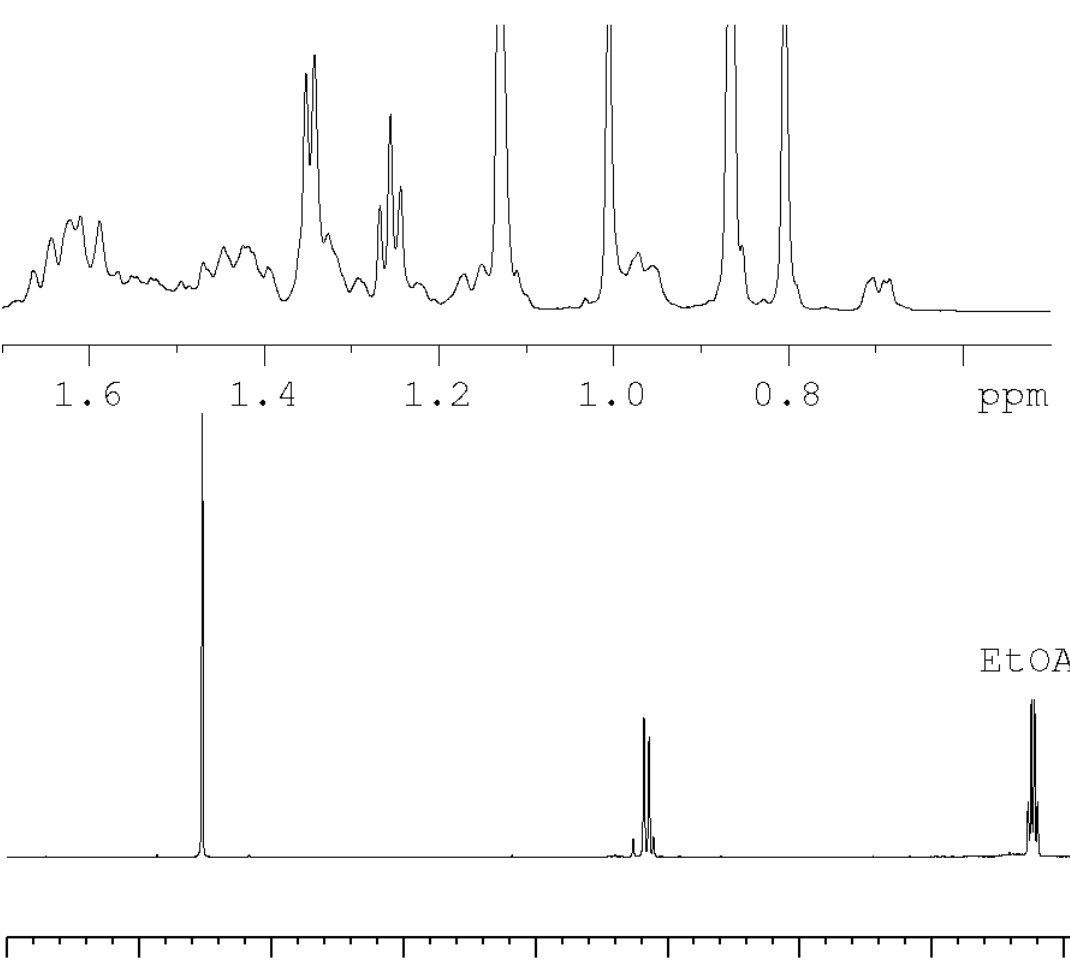

7.5

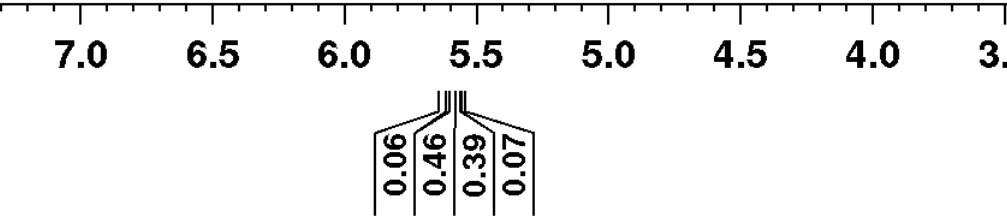

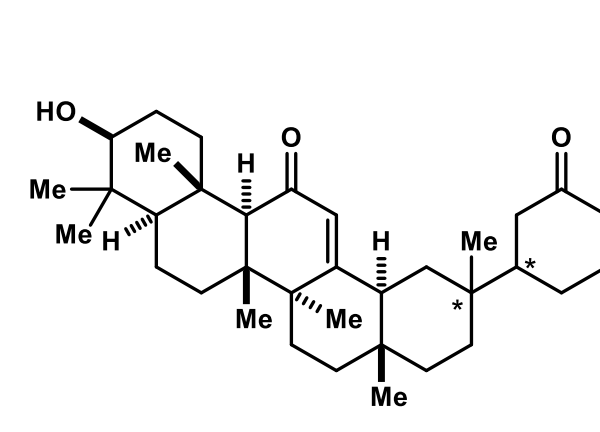

32
$8 \cdot 7 \cdot 1: 1$

$d r$

$$
\text { EtOAC }
$$

F2 - Acquisition Parame Date_ 20190610

INSTRUM PROBHD $5 \mathrm{~mm}$ CPBBO $\mathrm{BB}-$ PUIPROG zg30
98074 SOLVENT DS SWH FIDRES $\quad 9615.385$ $\begin{array}{ll}\mathrm{AQ} & 0.098042 \\ \mathrm{RG} & 5.0998478\end{array}$ RG

\section{$===$}

$\mathrm{SFO} 1$ $\mathrm{P} 1$

PLW1

52.000

13.70
298.0

0.10000000

CHANNET $f 1$ 600.1342009
$1 \mathrm{H}$ $1 \mathrm{H}$
2.00

F2 - Processing paramet SI $\begin{array}{ll}\text { SF } & 600.1300345\end{array}$ 


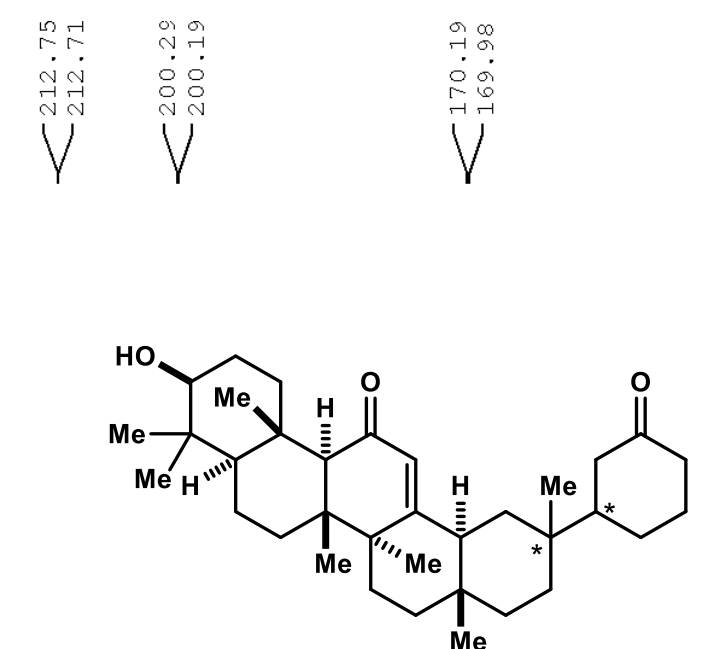

32

$8: 7: 1: 1 d r$

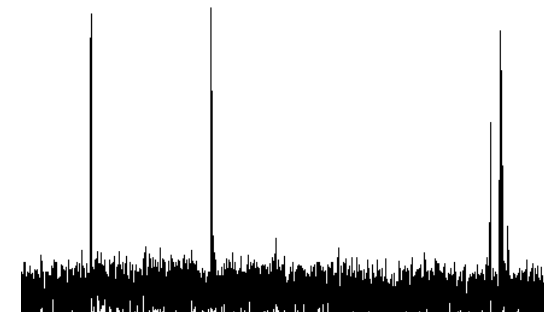

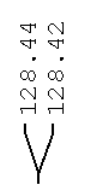

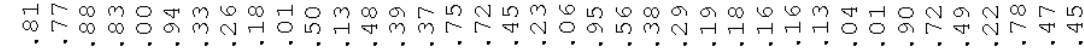
œ
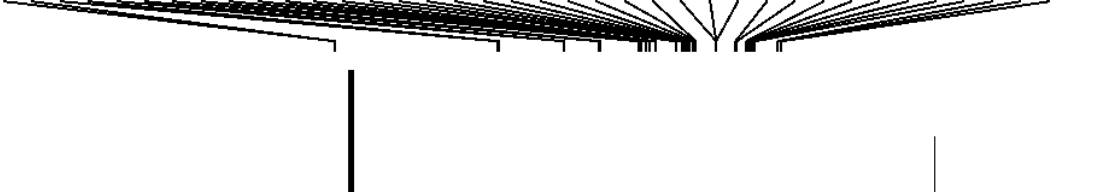

Time

av 600

$5 \mathrm{~mm}$ CPBBO $\mathrm{BB}-$

PULPROG

TD

SOLVENT

DS

SWH

FIDRE

AQ

DW

DE

D1

D11

$====$
SFO1

$\mathrm{SEO} 1$
NUC1

N1

PLW1

$===$
SEO2
NUC2

CPDPRG [2

PCPD2
PLW2

PLW12

30.00000000
0.63095999

F2 - Processing paramet

SI 65536

$\begin{array}{lr}\text { SI } & 65536 \\ \text { SF } & 150.9028085 \\ \text { WDW } & \text { EM }\end{array}$

SSB

LB

$\mathrm{PC}$

36231.883 0.552855
0.9043968 2050
13.800

19.63

0.40000001

0.03000000

CHANNEL $f 1===$

150.9194080
$13 \mathrm{C}$

64.00000000

CHANNEL $f 2===$ $1 \mathrm{H}$

80.00

0

1.00

1.00

$$
\text { EtOAC }
$$

EtOAC

60

40

20

ppm 


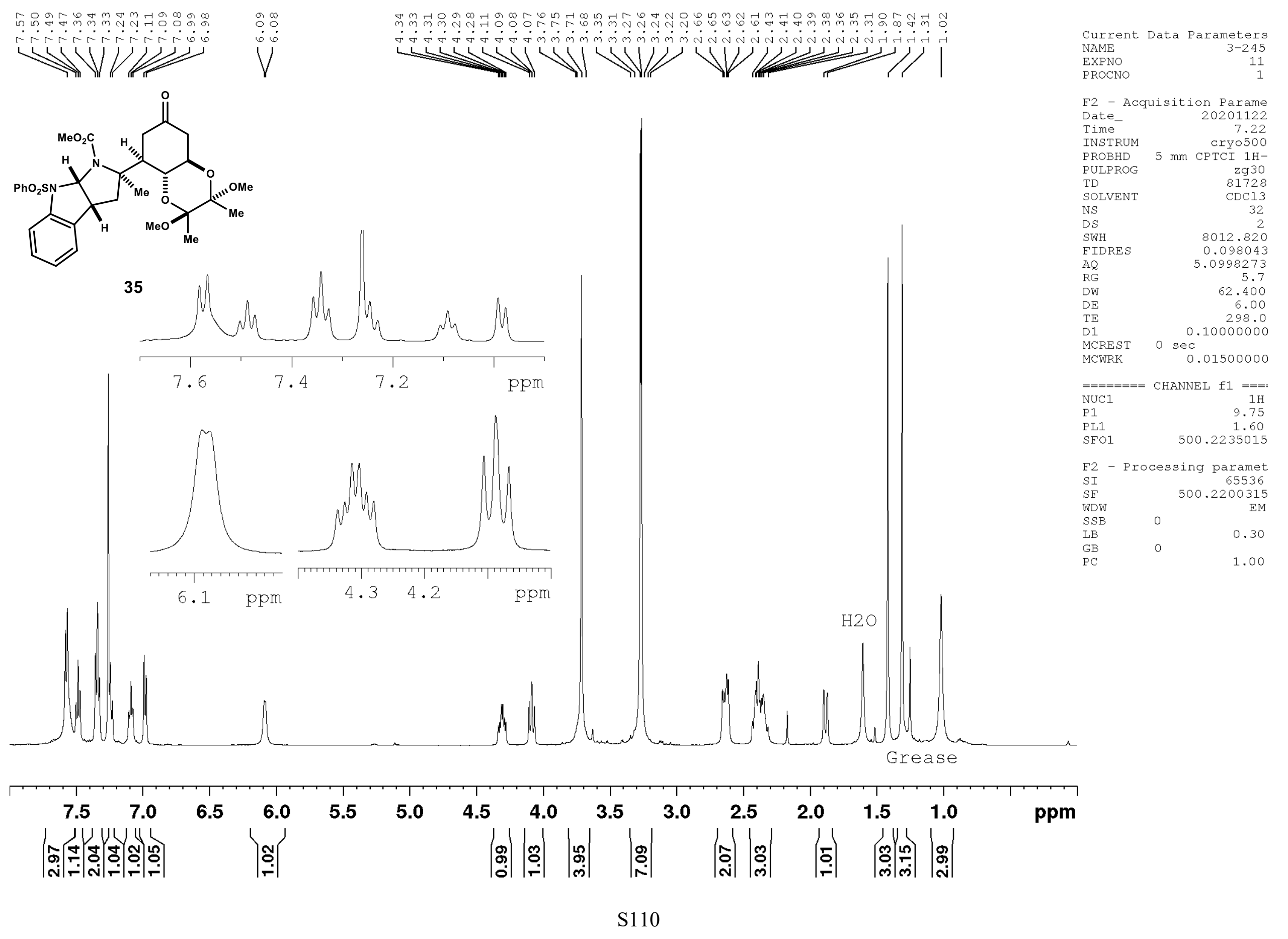




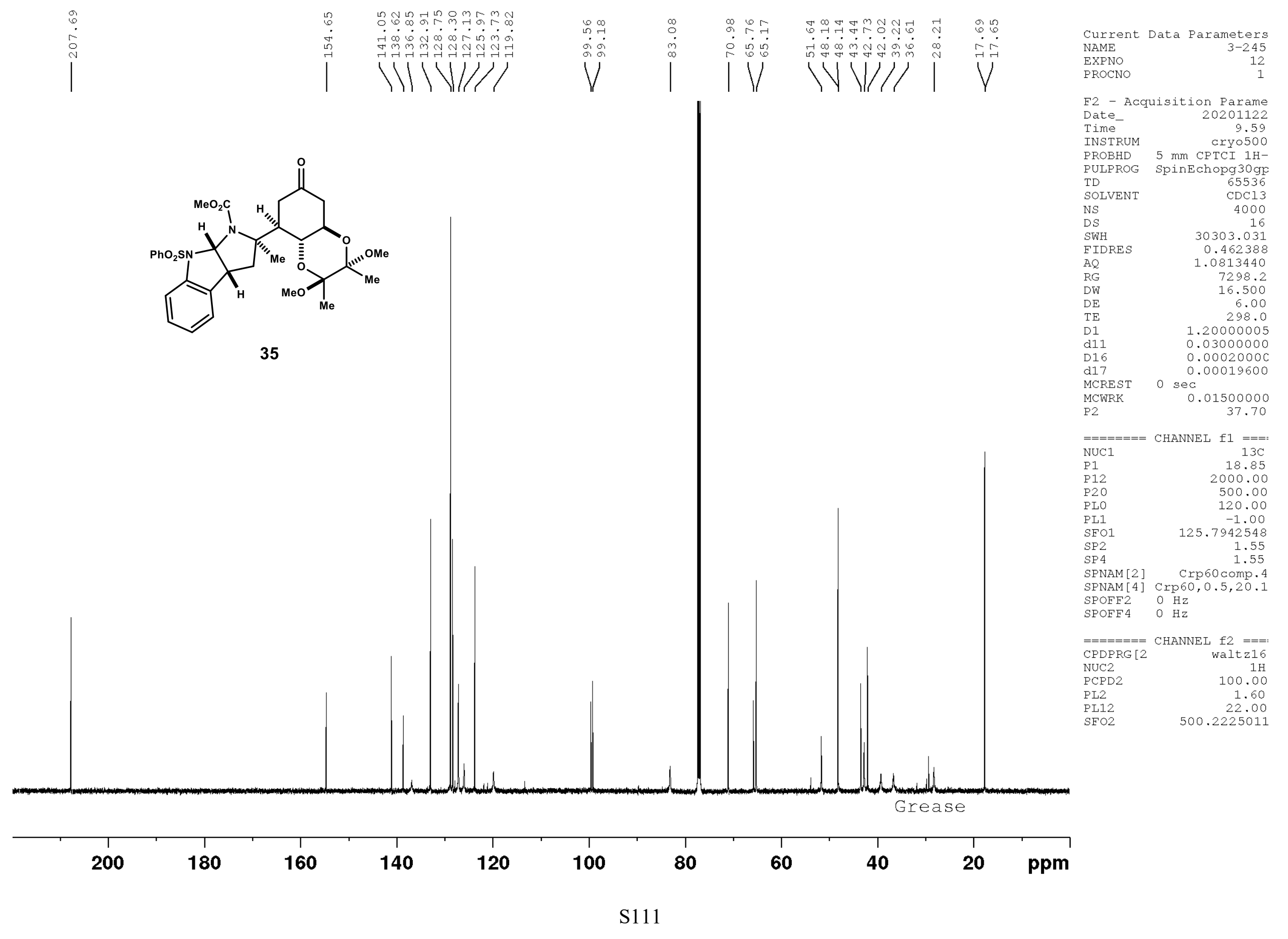




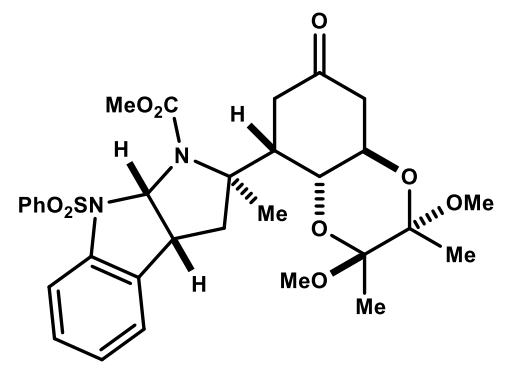

S17
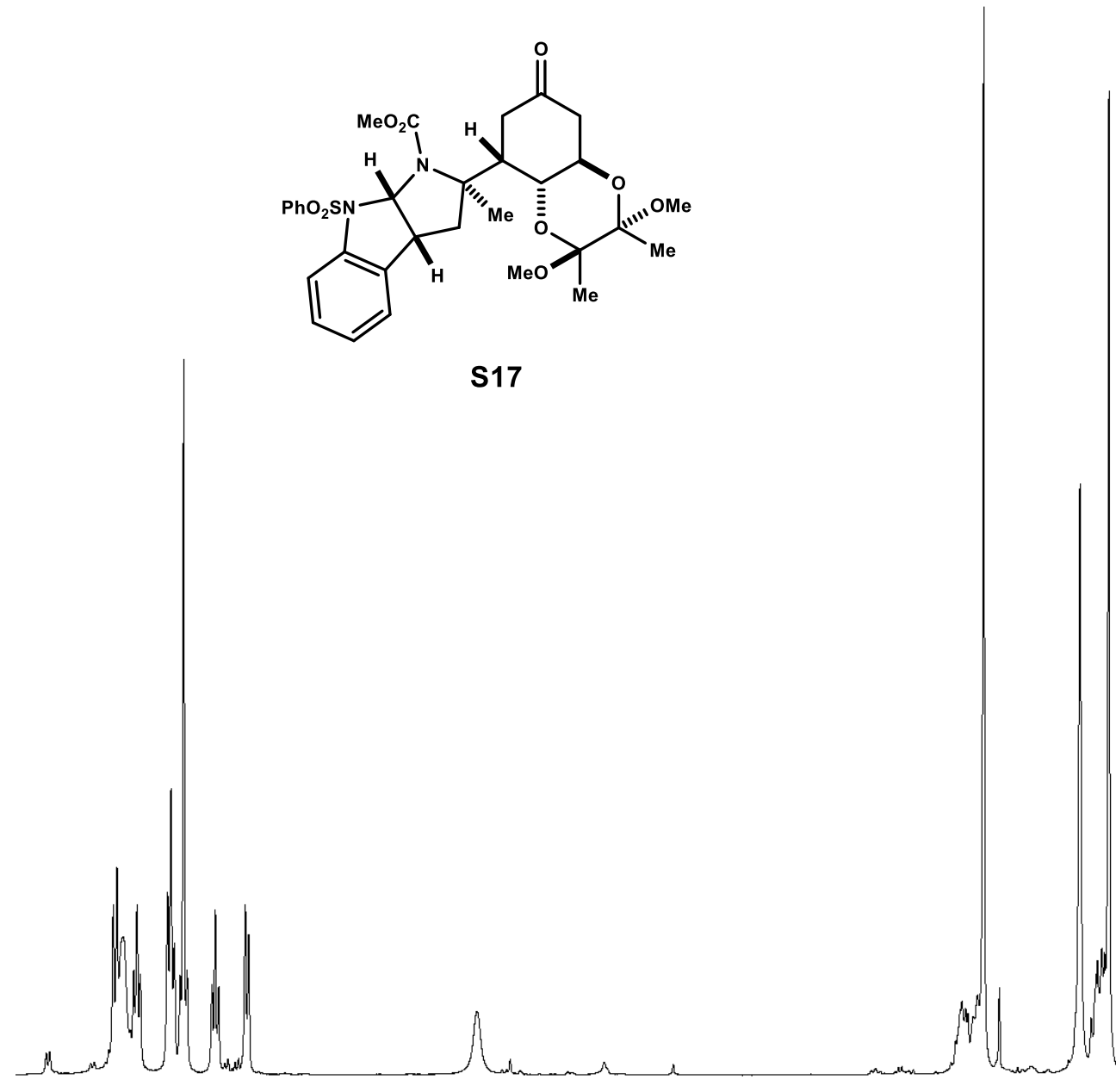

F2 - Acquisition Parame Date

Time

INSTRUM

PROBHD$$
\text { TD }
$$

TD
SOLVENT

NS

DS

$\begin{array}{lr}2 \\ \text { SWH } & 8012.820\end{array}$ 0.098043
5.0998273

F2 - Processing paramet SI $\quad 65536$ 


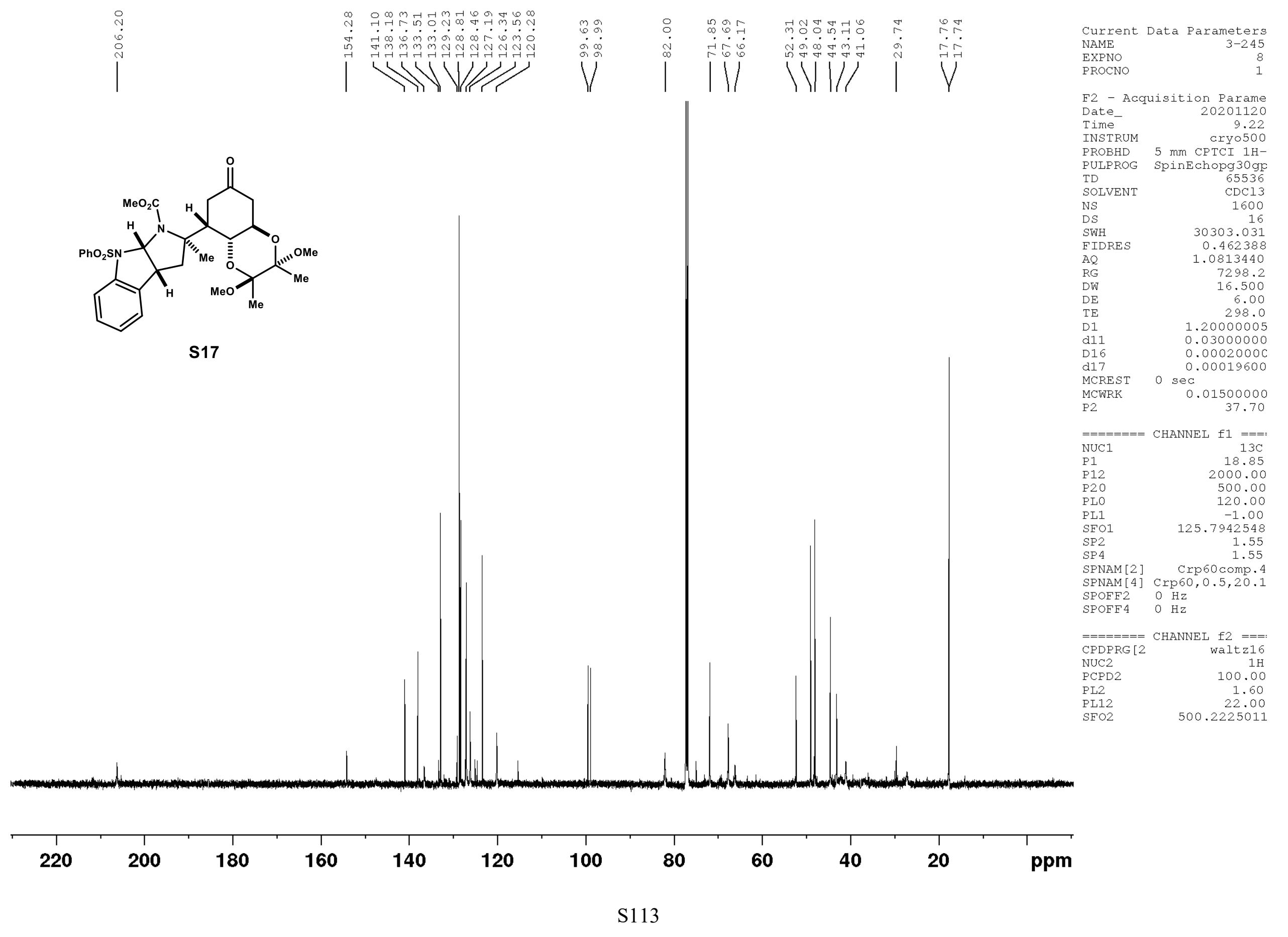


Tं Tं

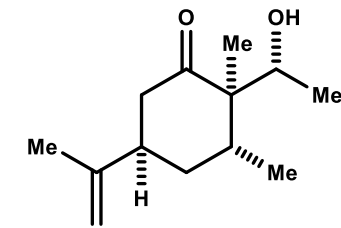

S18

F2 - Acquisition Parame Date_ 20200319
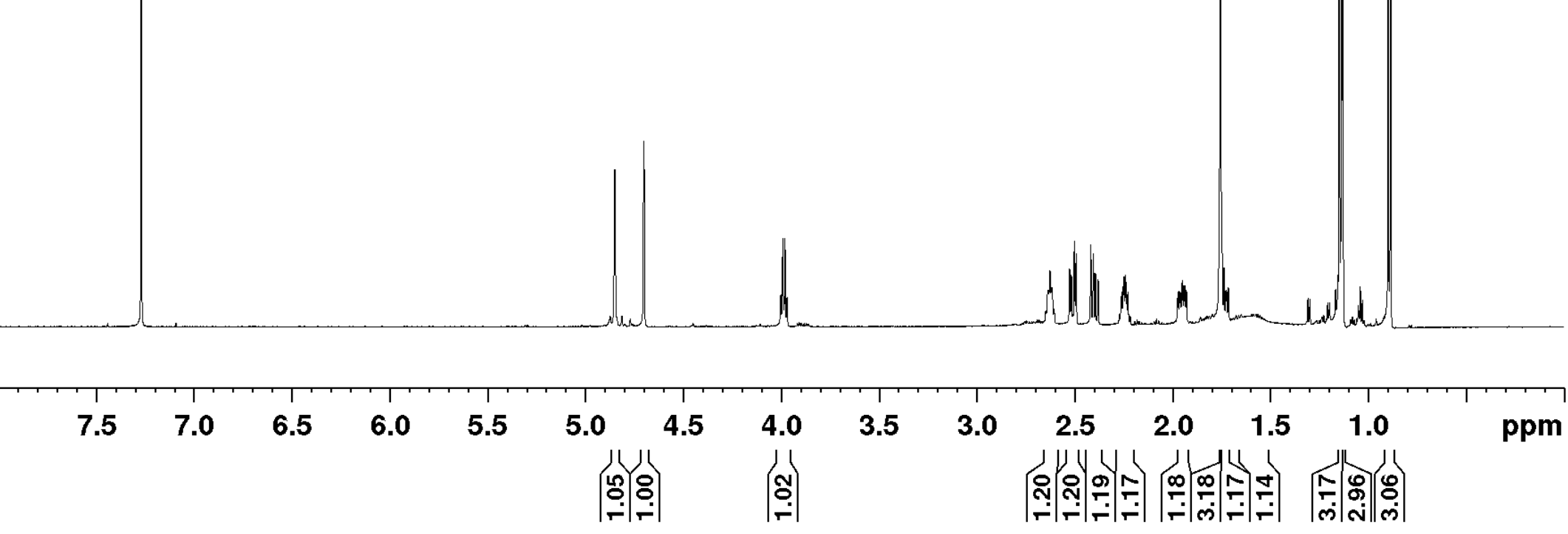


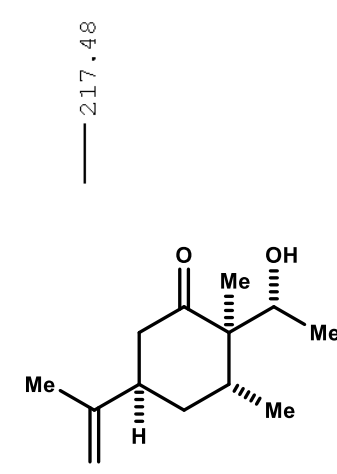

S18
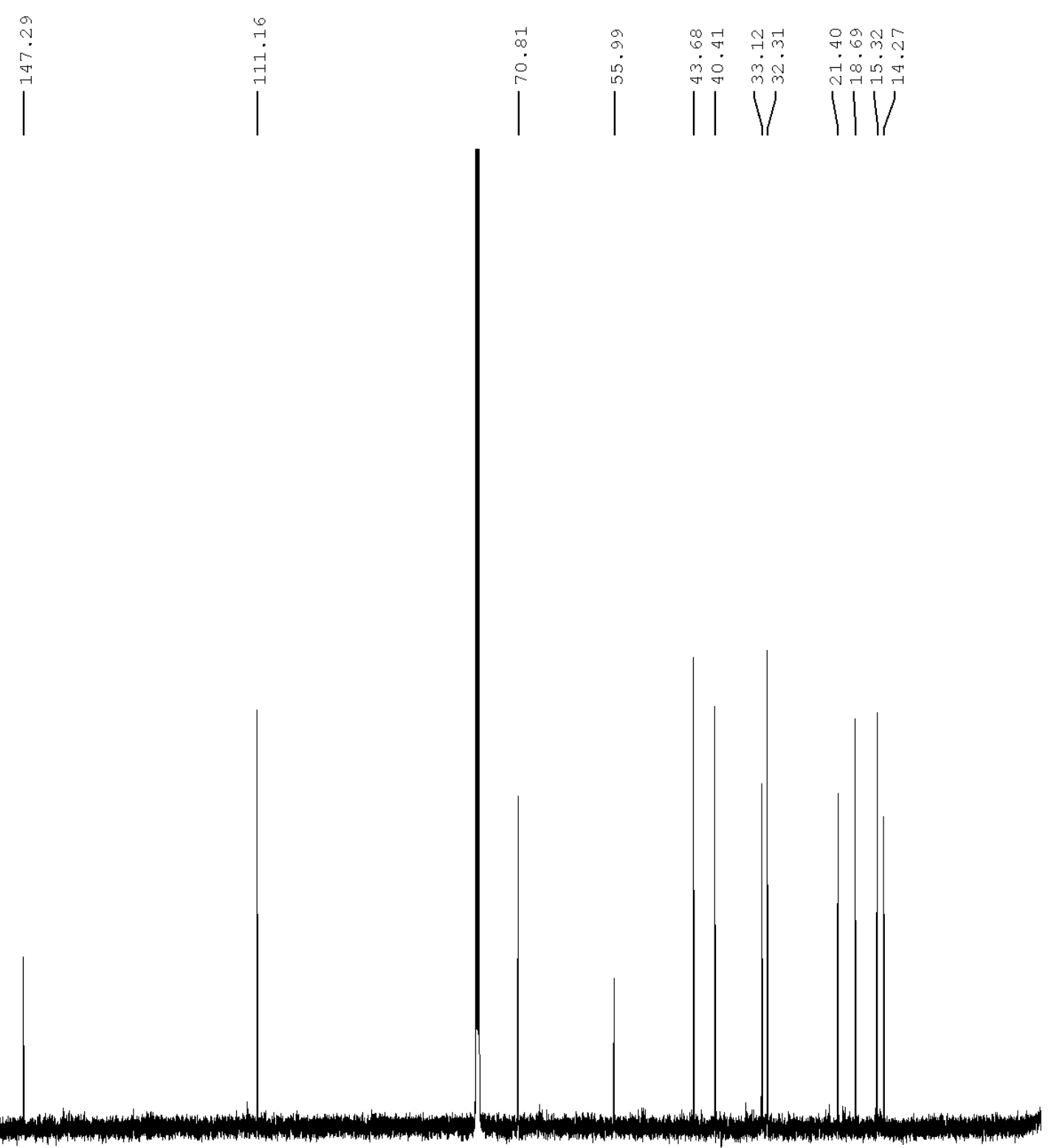


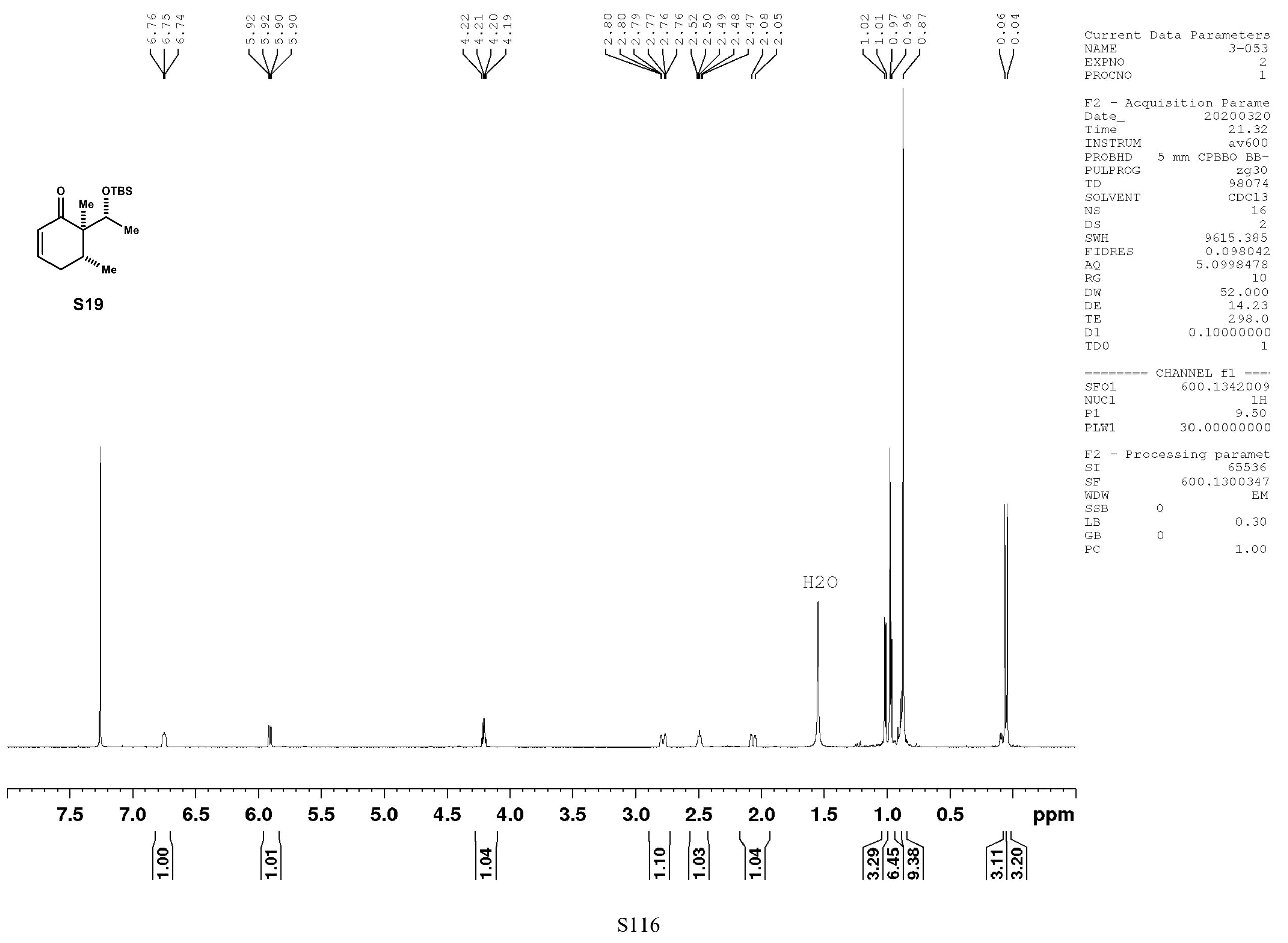



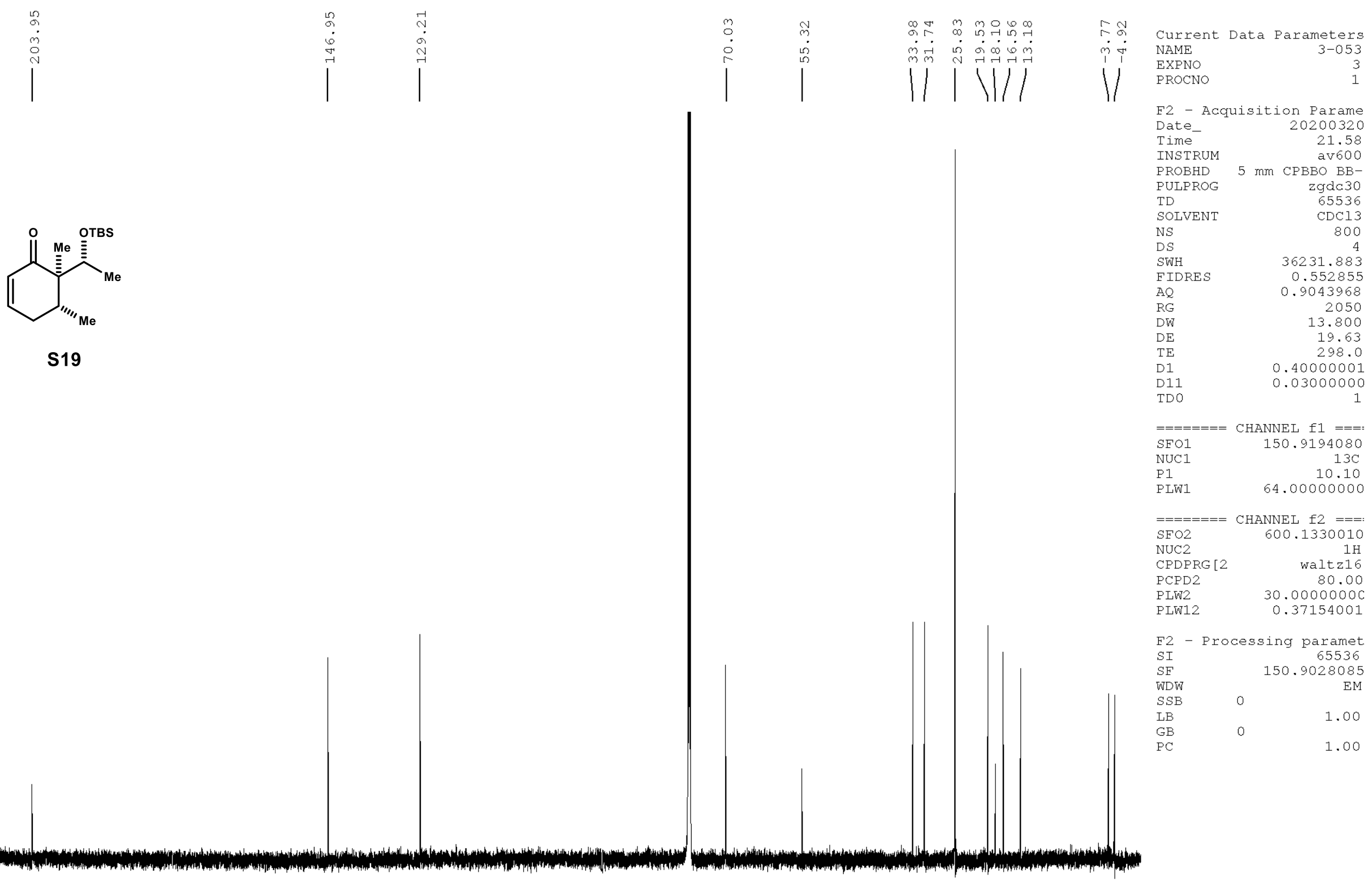

\begin{tabular}{rrrrrrrrrrrrr}
\hline 200 & 180 & 160 & 140 & 120 & 100 & 80 & 60 & 40 & 20 & ppm
\end{tabular}



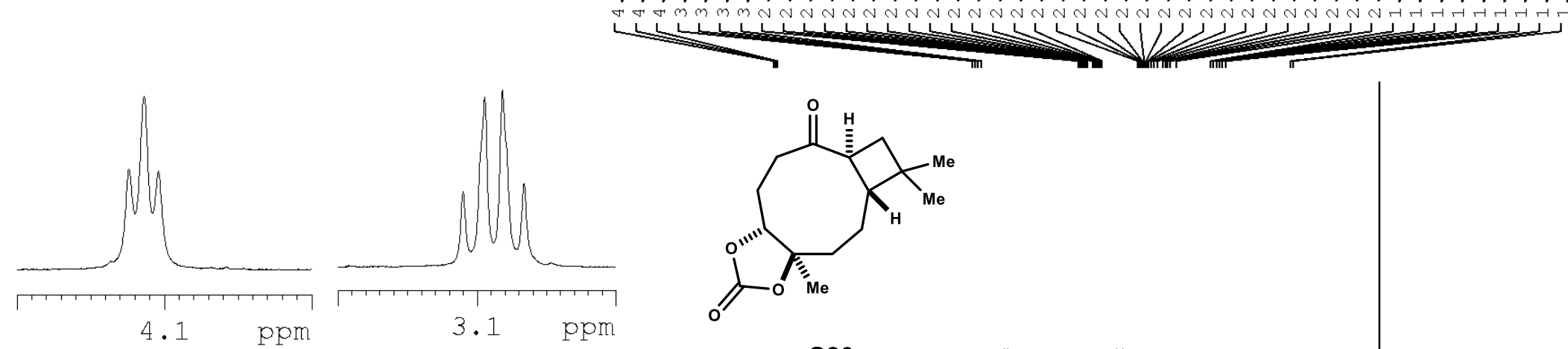

S20
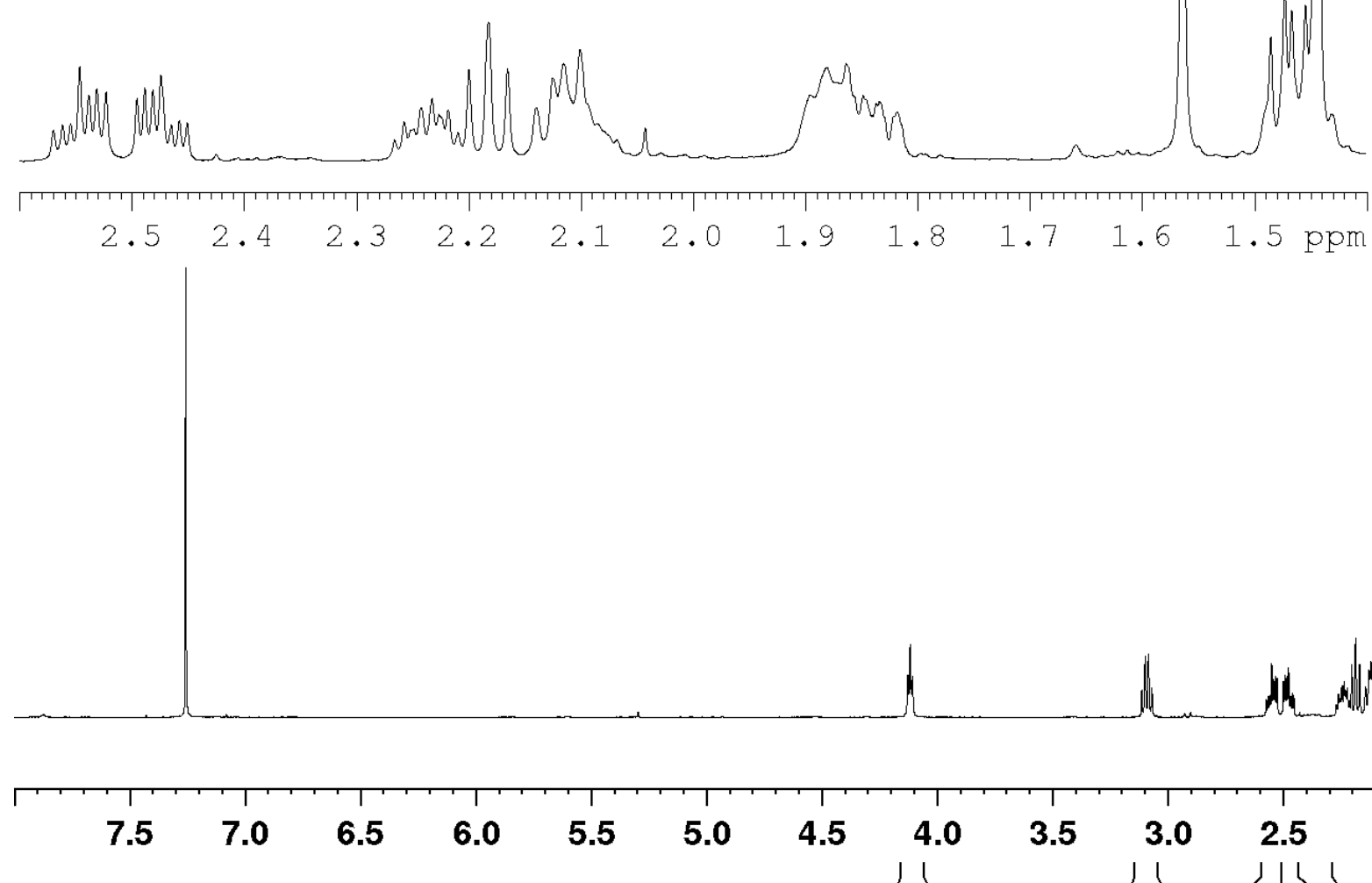

$\left|\begin{array}{l}0 \\ 8 \\ \hdashline\end{array}\right|$

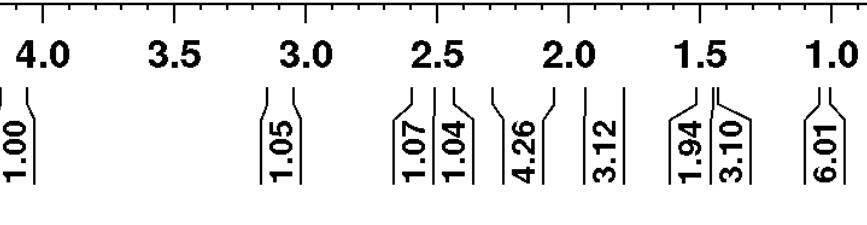

S118 
<smiles>CC1(C)C[C@@]2(C)C(=O)CC[C@@H]3CC(=O)O[C@@]3(C)CC[C@]12C</smiles>

S20
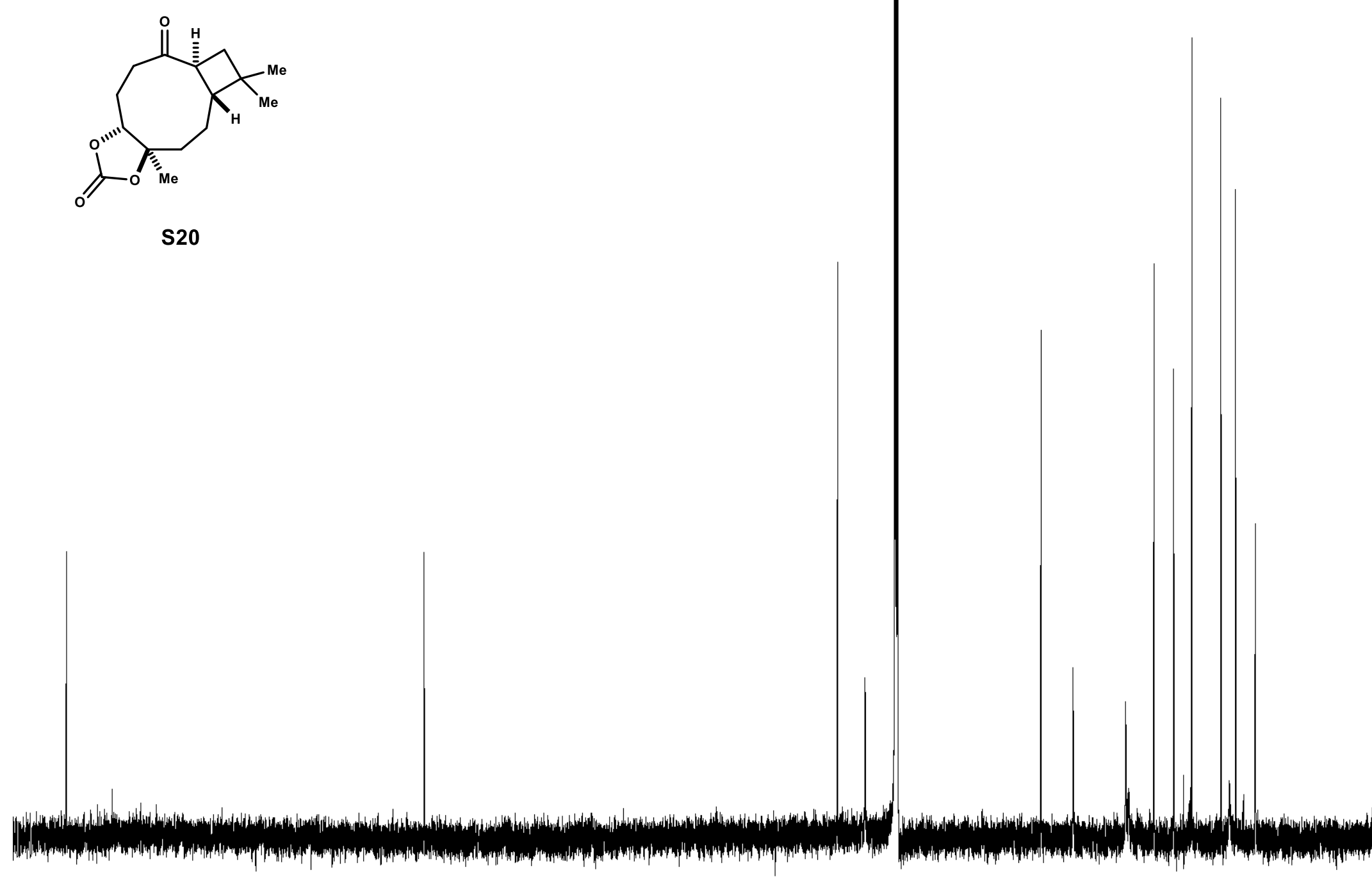

F2 - Acquisition Parame Date

INSTRUM

$5 \mathrm{~mm}$ CPBBO $\mathrm{BB}$

PULPROG

TD

NS

DS

RG

DW

TE

D1

TDO

$====$
SFO1

NUC1

P1

0.9043968

2050
13.800

19.63

1.20000005

0.03000000

CHANNEL $\mathrm{f} 1===$

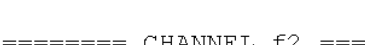

SFO2 600.1330010 600.1330010
$1 \mathrm{H}$

CPDPRG [2 waltz16

PCPD2 30.00000000

PLW12

0.37154001

F2 - Processing paramet

SI 65536

SF $\quad 150.9028085$

WDW

LB

GB

1.00

1.00

\begin{tabular}{rrrrrrrrrrr}
\hline 200 & 180 & 160 & 140 & 120 & 100 & 80 & 60 & 40 & 20 & ppm
\end{tabular}



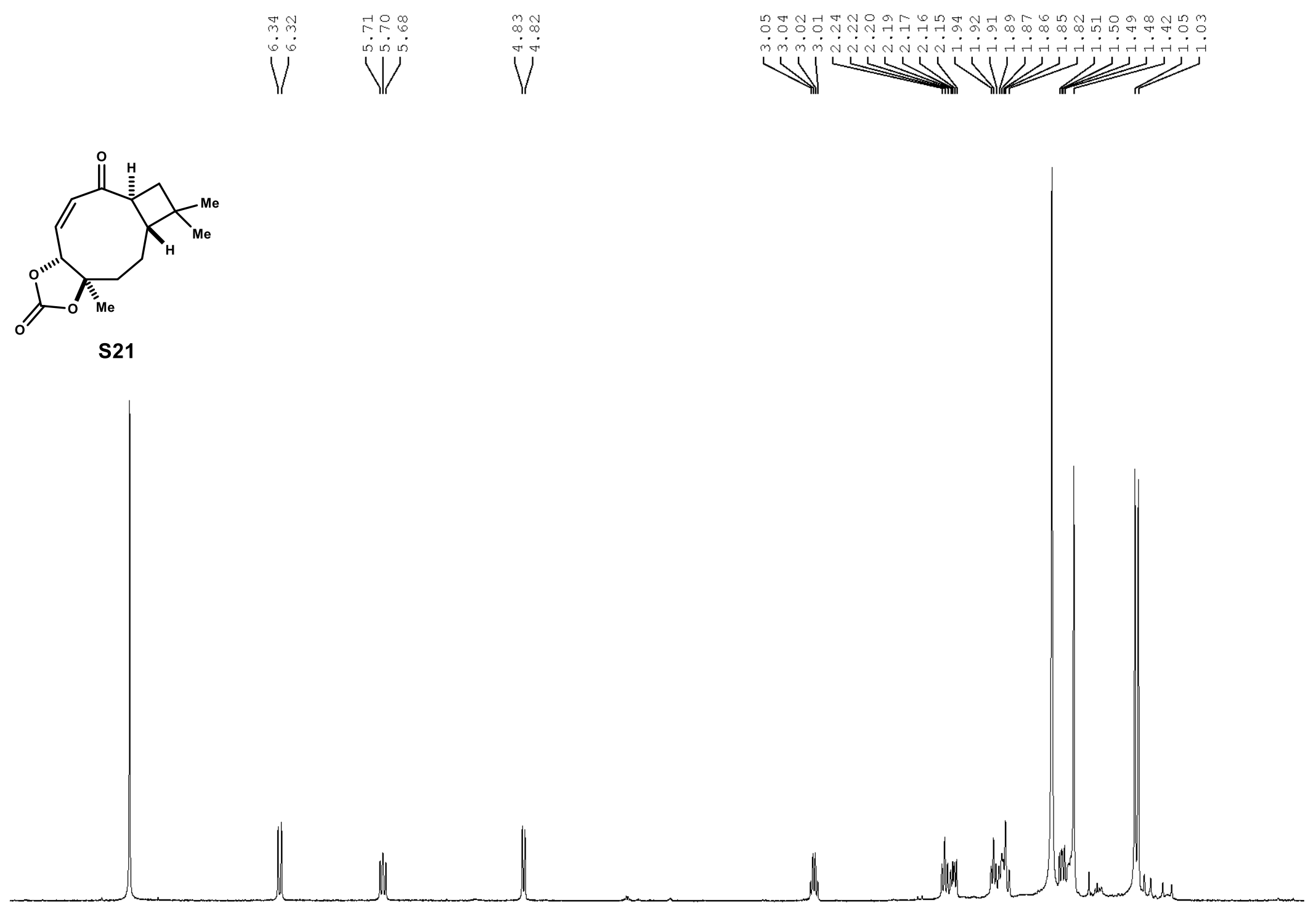

Current Data Parameters

$$
\text { NAME }
$$

PROCNO

F2 - Acquisition Parame Date_

Time

PROBHD

PULPROG

TD
SOLVENT

NS

$\begin{array}{lr}\text { DS } & 2 \\ \text { SWH } & 9615.385\end{array}$

AQ $\quad 5.0998478$

RG

DW

TE

TDO

20201021
8.43

$========$ CHANNEL $\mathrm{fl}===$

SFO1 600.1342009

NUC1

P1

$1 \mathrm{H}$
9.50

PLW1 $\quad 30.00000000$

F2 - Processing paramet

SI $\quad 65536$

$\begin{array}{lr}\text { SF } & 600.1300350 \\ \text { WDW } & \text { EM }\end{array}$

0.30

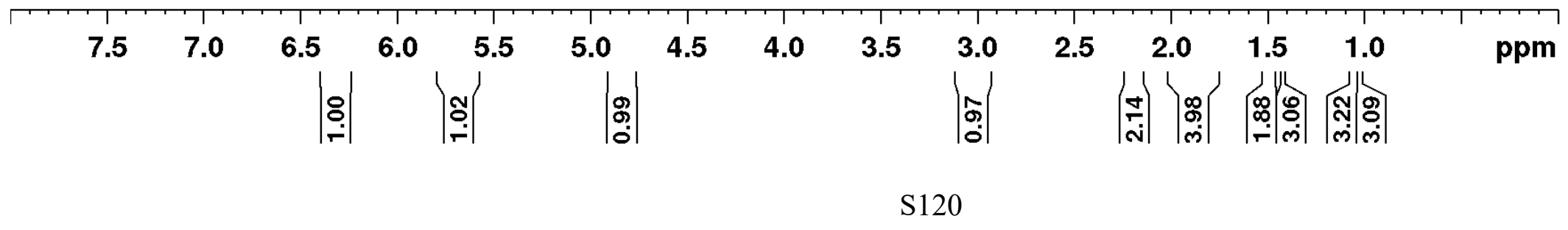




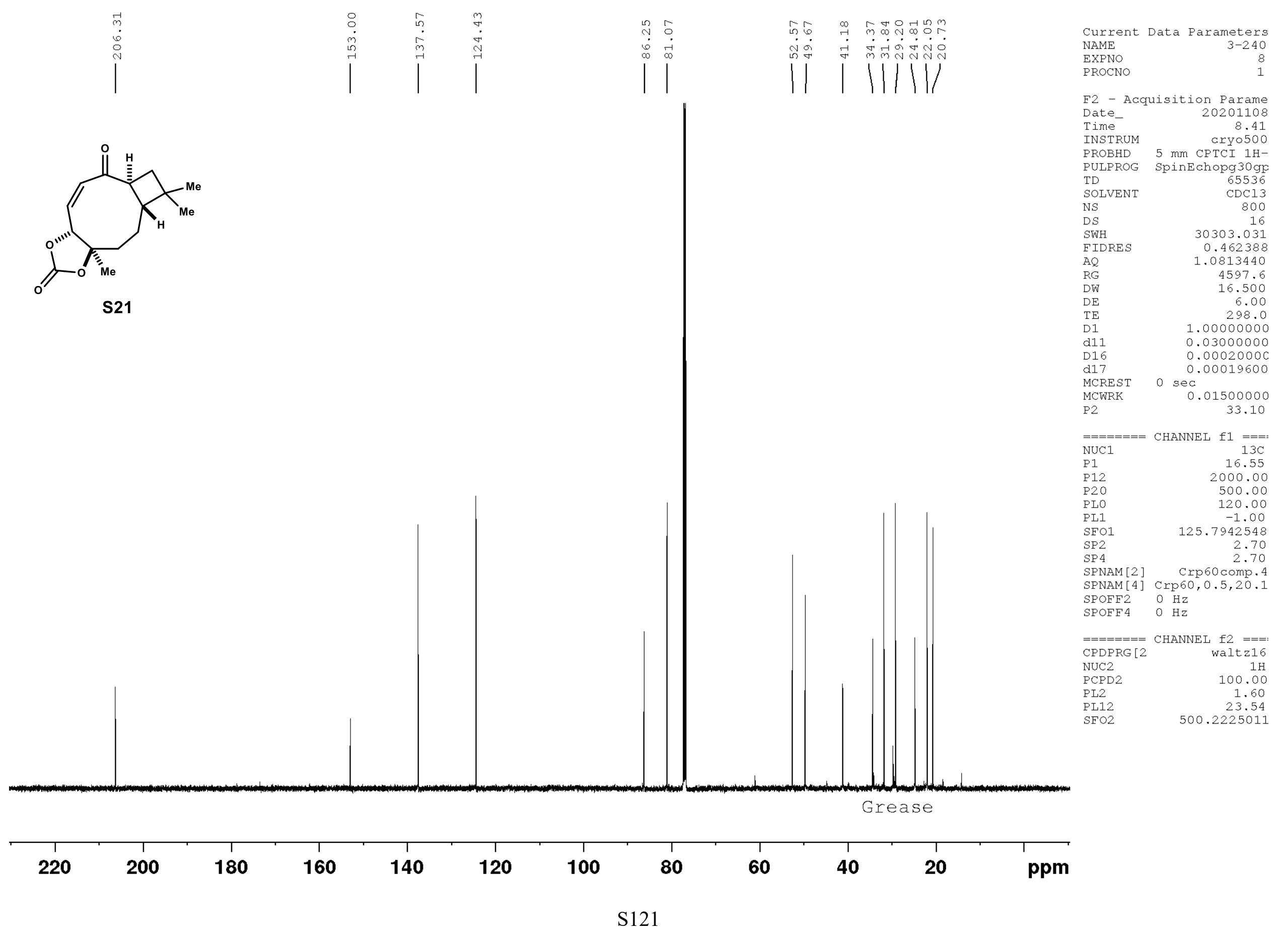



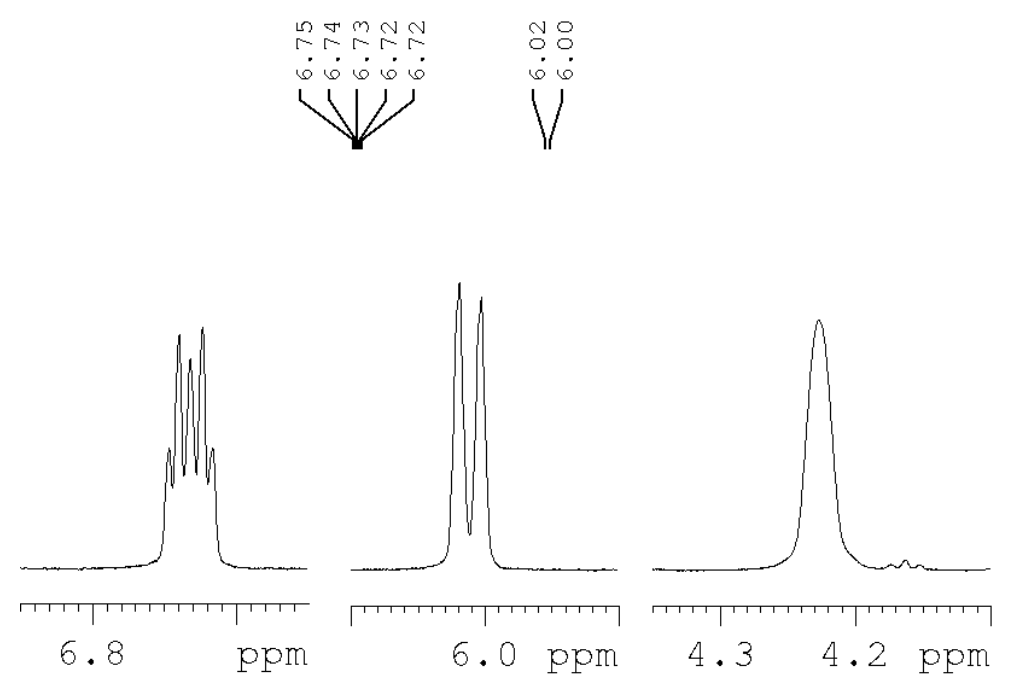

$6.0 \mathrm{ppm}$

$$
\text { 4.3 4.2 ppm }
$$
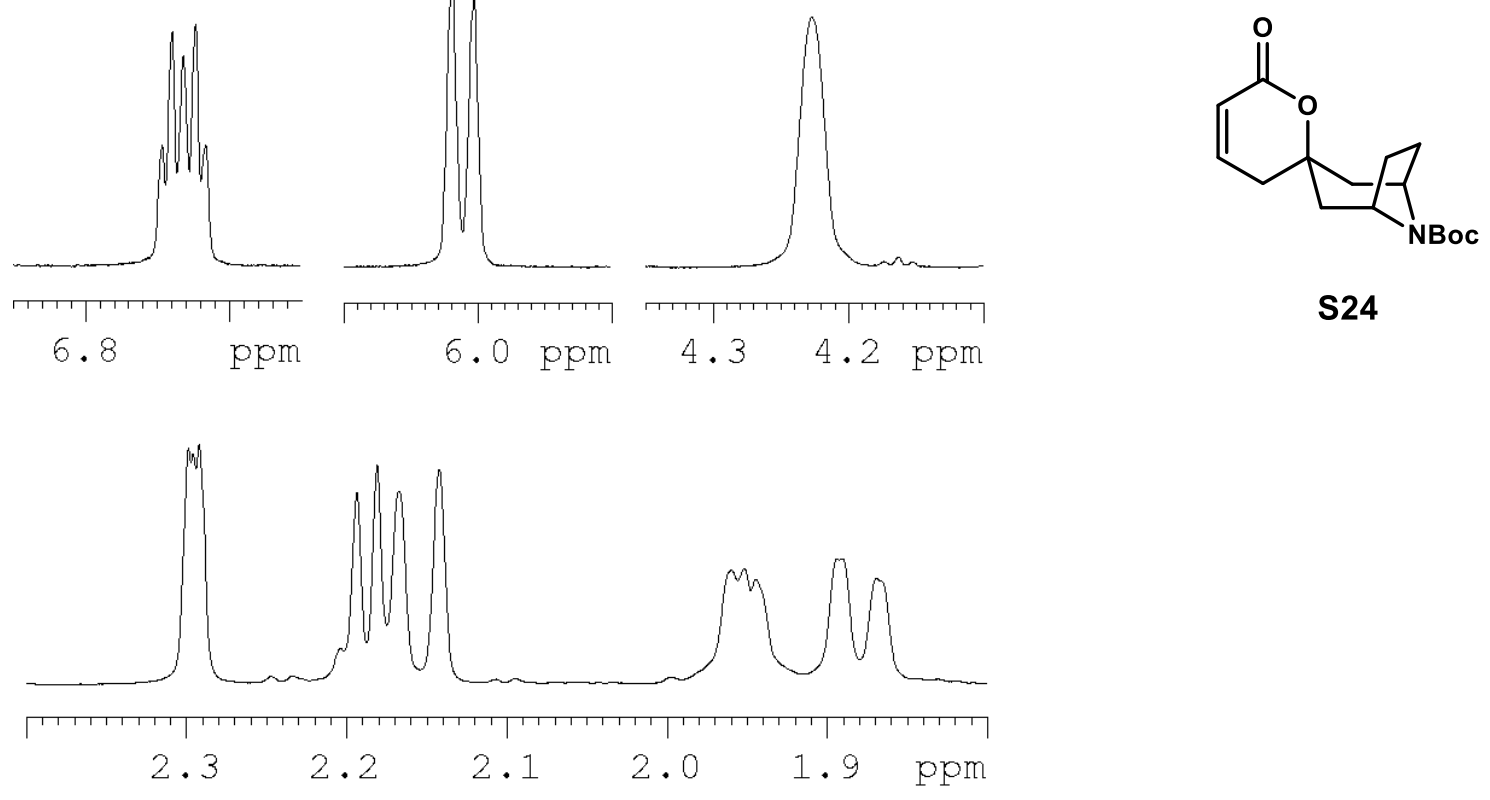

S24
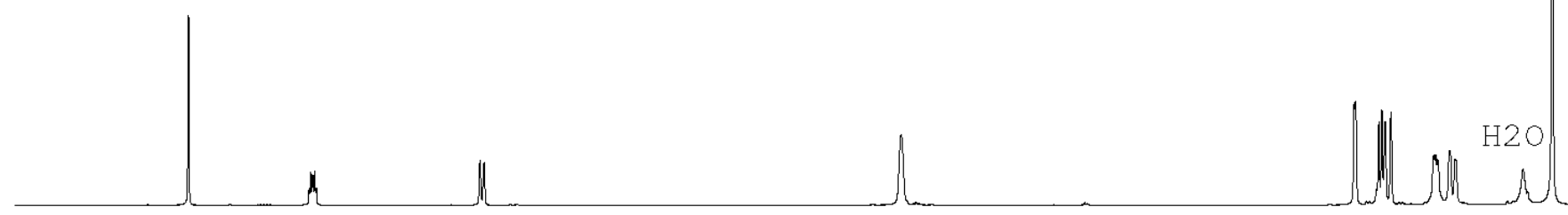

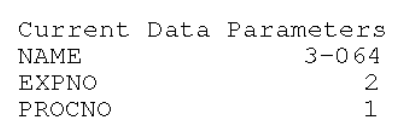

F2 - Acquisition Parame Date

Time

$5 \mathrm{~mm}$ CPBBO $\mathrm{BB}-$

PULPROG

SOLVENT

NS

$\begin{array}{lr}\text { DS } & 2 \\ \text { SWH } & 9615.385 \\ \text { FIDRES } & 0.098042\end{array}$

$\mathrm{AQ}$

RG
DW

DE

D1
TDO

$====$

$\mathrm{SFO} 1$
NUC1

NUC1

P1 1

F2 - Processing paramet

SI 65536

SF $\quad 600.1300348$

WDW

LB

PC 5.0998478 10
52.000
14.23 14.23
298.0 0.10000000 41 600.1342009 $\frac{1 \mathrm{H}}{9}$

0.30 1.00

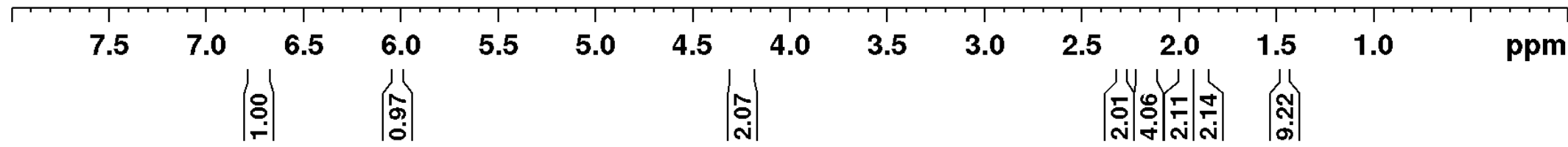



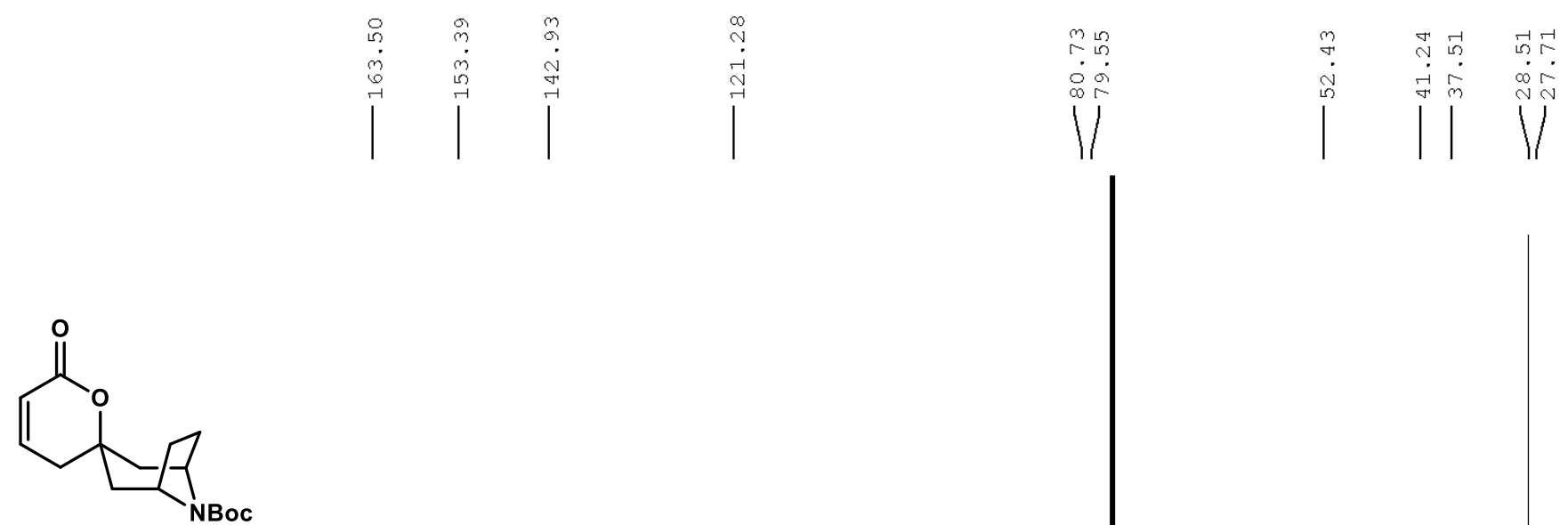
isition Parame Date_

Time

PROBHD

PROBHD

TD

SOLVENT

NS

$\begin{array}{lr}\text { DS } & 4 \\ \text { SWH } & 36231.883\end{array}$

0.552855

RG

$\mathrm{DE}$

TE

D1
D11

S24

TDO

$=====$

$\mathrm{SFO1}$

NUC1

P1
PLW1

av 600

av 600

zgdc 30

$======\mathrm{CH}$ (1)

$\mathrm{SFO} 2$

NUC2

CPDPRG
PCPD2

PLW2 2

PLW12

0.9043968

2050
13.800

19.63
298.0

0.40000001

0.03000000

CHANNEL $\mathrm{f} 1===$

150.9194080
$13 \mathrm{C}$

64.00000000

F2 - Processing paramet

SI 65536

$\begin{array}{ll}\text { SF } & 150.9028085\end{array}$

WDW

LB

GB

$\mathrm{PC}$

1.00

1.00

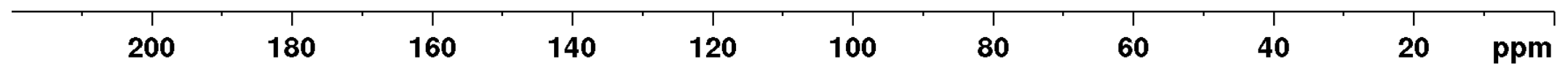




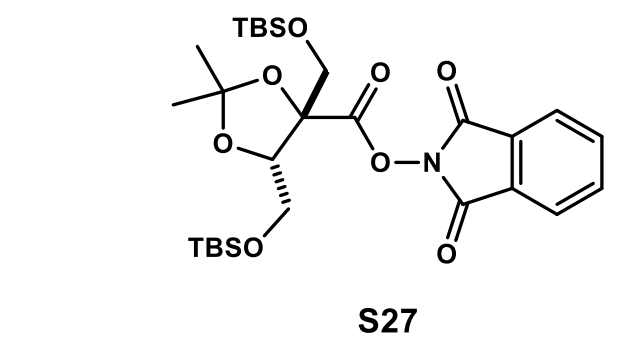

F2 - Acquisition Parame Date

Time

INSTRUM

PROBHD

PUT

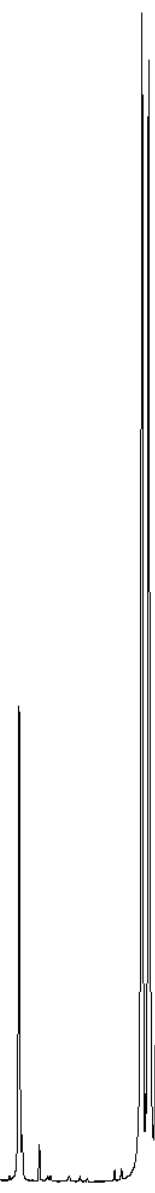

TD
SOLVENT

NS

DS

FIDRES

$\mathrm{AQ}$
$\mathrm{RG}$

RG

DW
$\mathrm{DE}$
$\mathrm{TE}$

$\mathrm{TE}$
$\mathrm{D} 1$

0.10000000

MCRES

MCWRK

0.01500000

$====$
NuC1

P1

CHANNEL f1 $===:$

1.60

F2 - Processing paramet

F2 - Processing paranet

$\begin{array}{lr}\text { SI } & 65536 \\ \text { SF } & 500.2200314\end{array}$
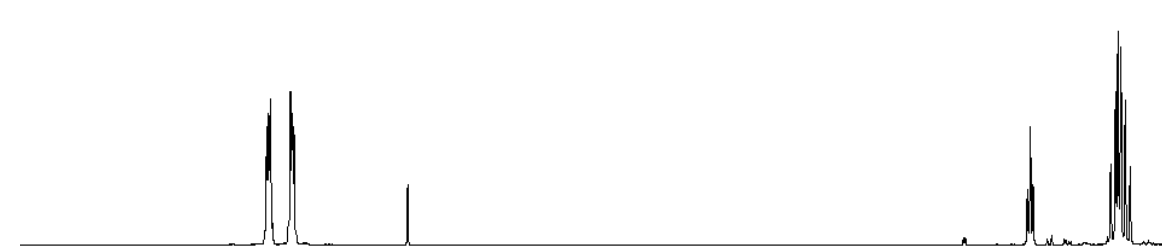

6

5

$\left|\begin{array}{l}0 \\ \hdashline \\ \hdashline\end{array}\right|\left|\begin{array}{c}0 \\ 0 \\ \dot{q}\end{array}\right|$

3

2

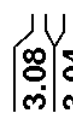

ppm

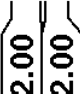




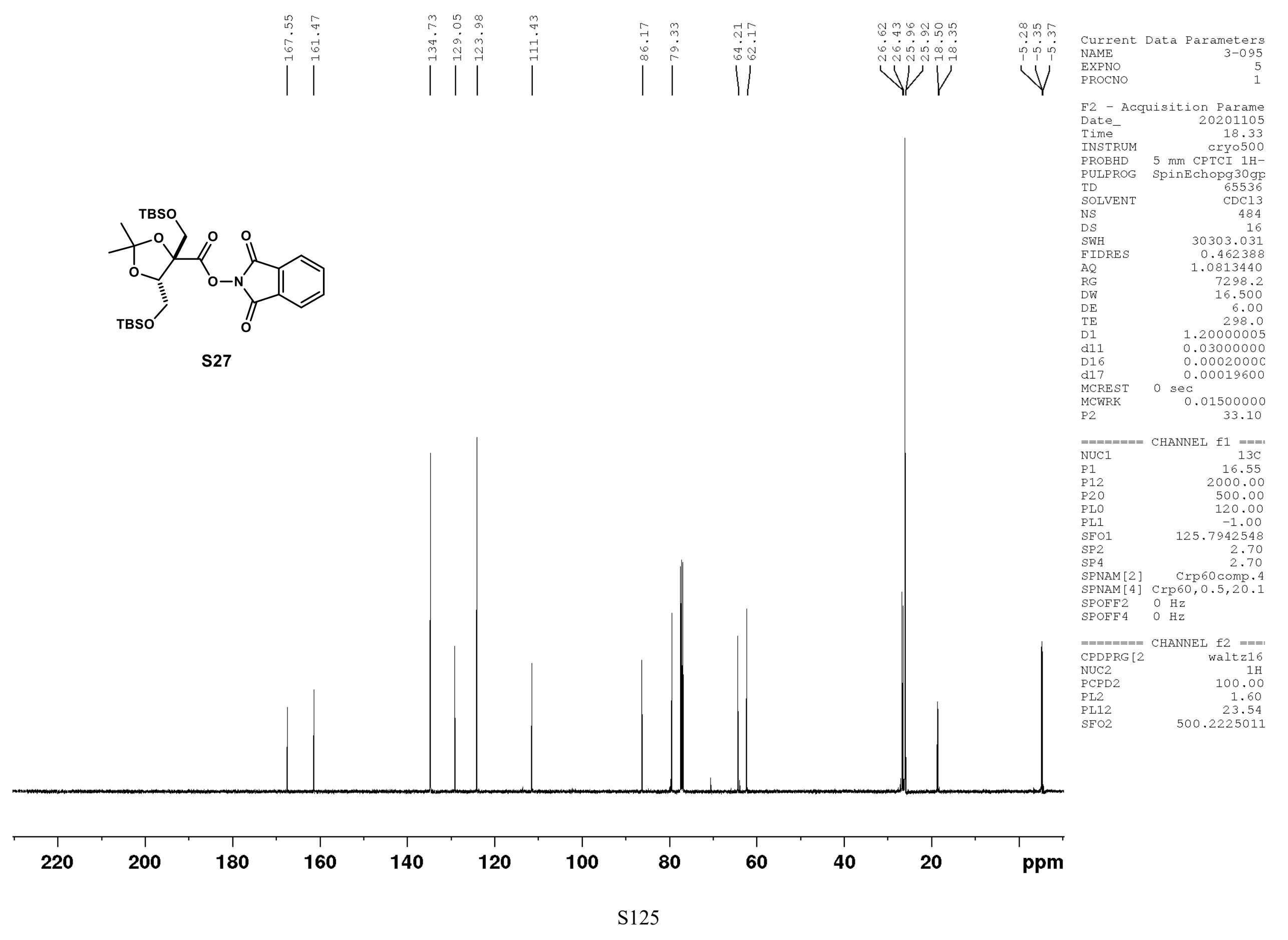




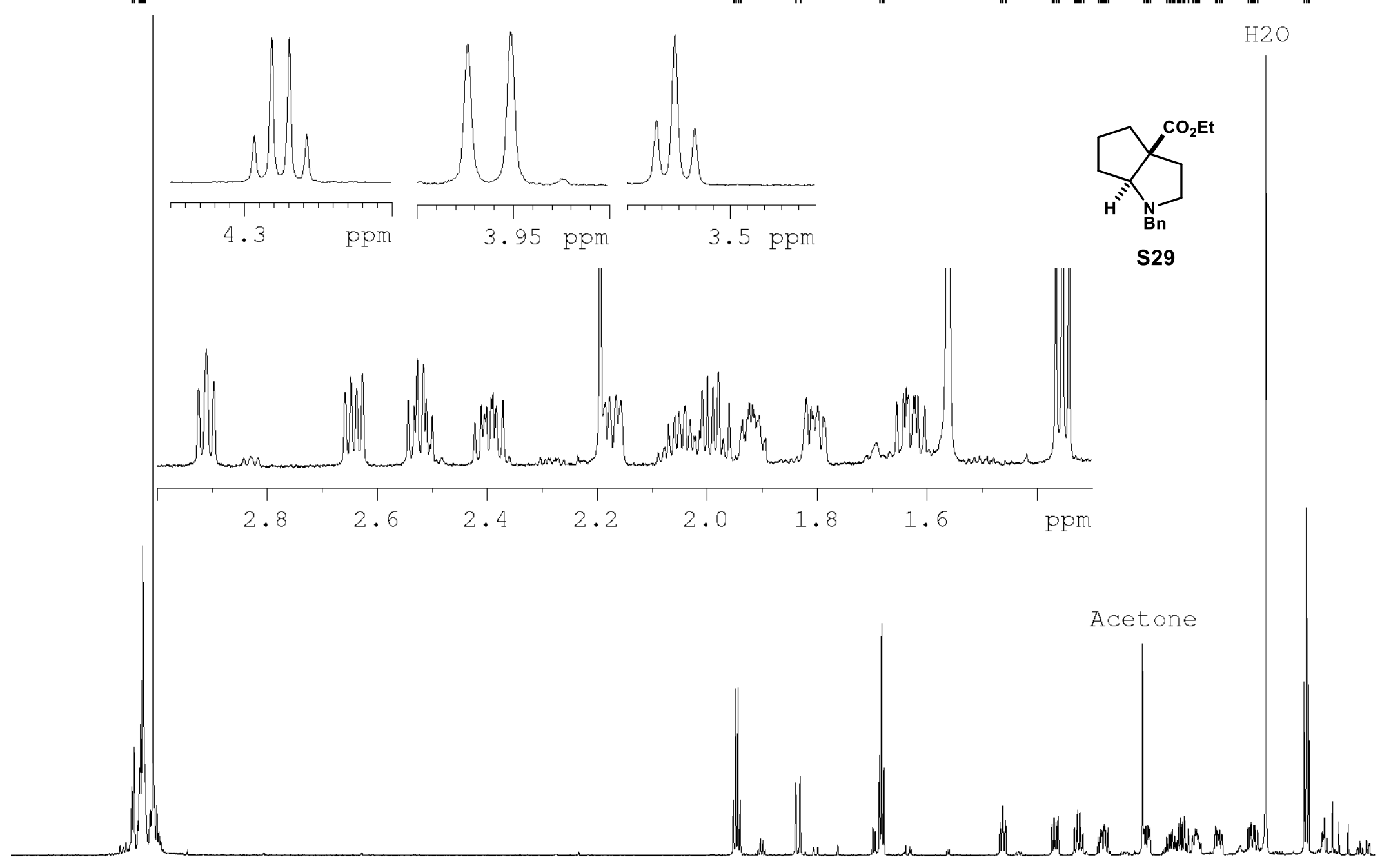

F2 - Acquisition Parame Date_ 20200303 14.54

$5 \mathrm{~mm}$ CPBBO $\mathrm{BB}-$ PUTPG $\mathrm{zg} 30$
98074 SOLVENT CDC13

DS

SWH $\quad 9615.385$ 0.098042
AO

2 - Processing paramet

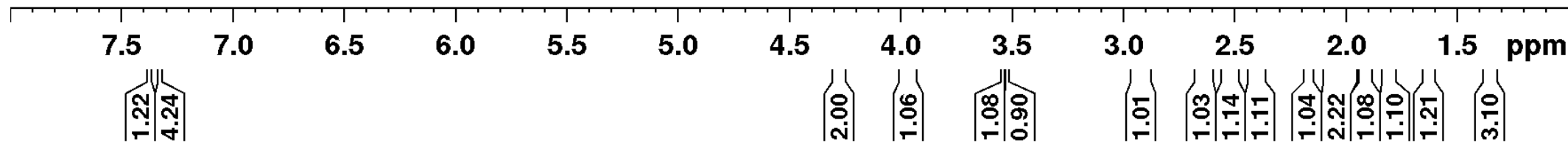




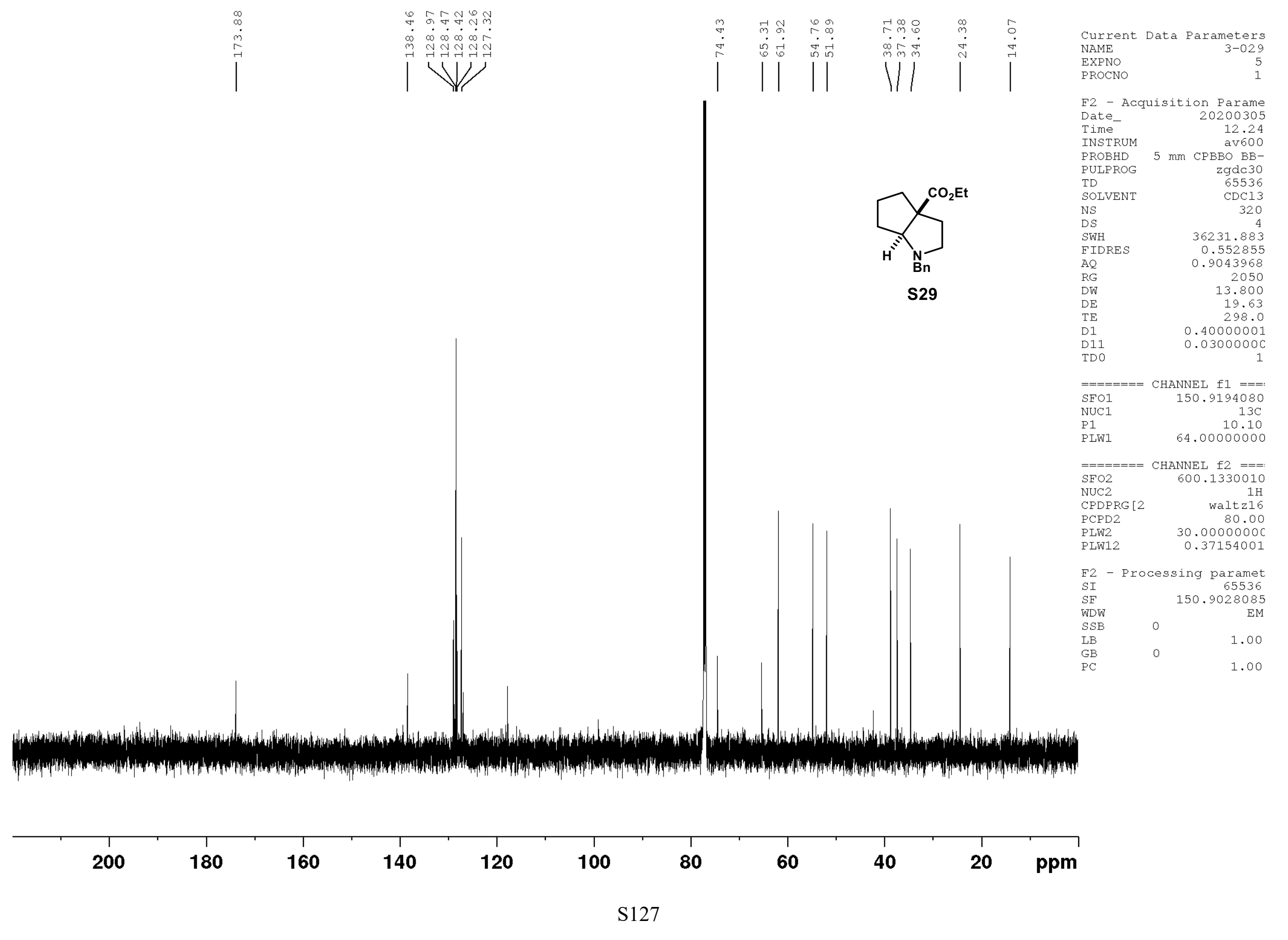




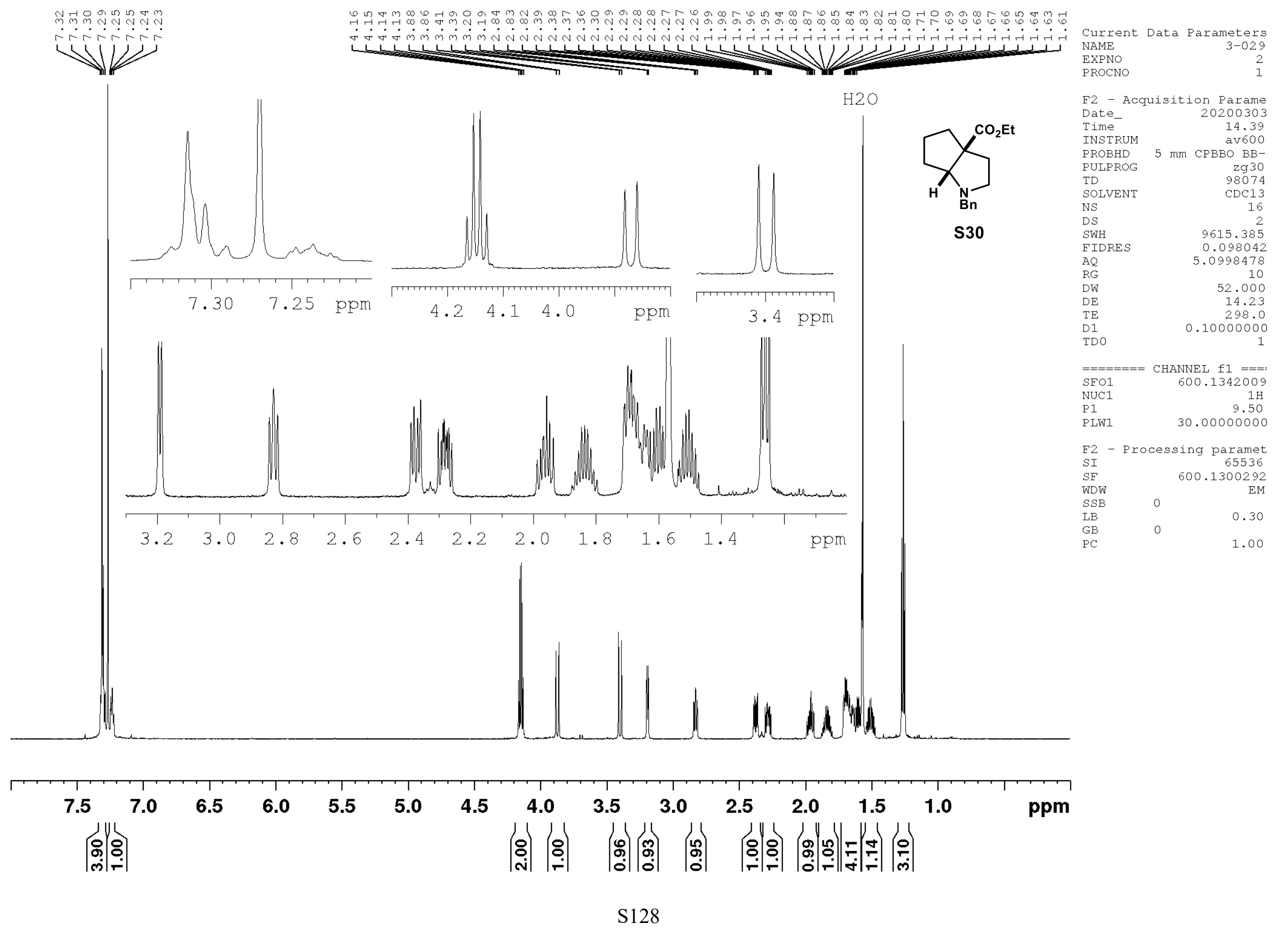




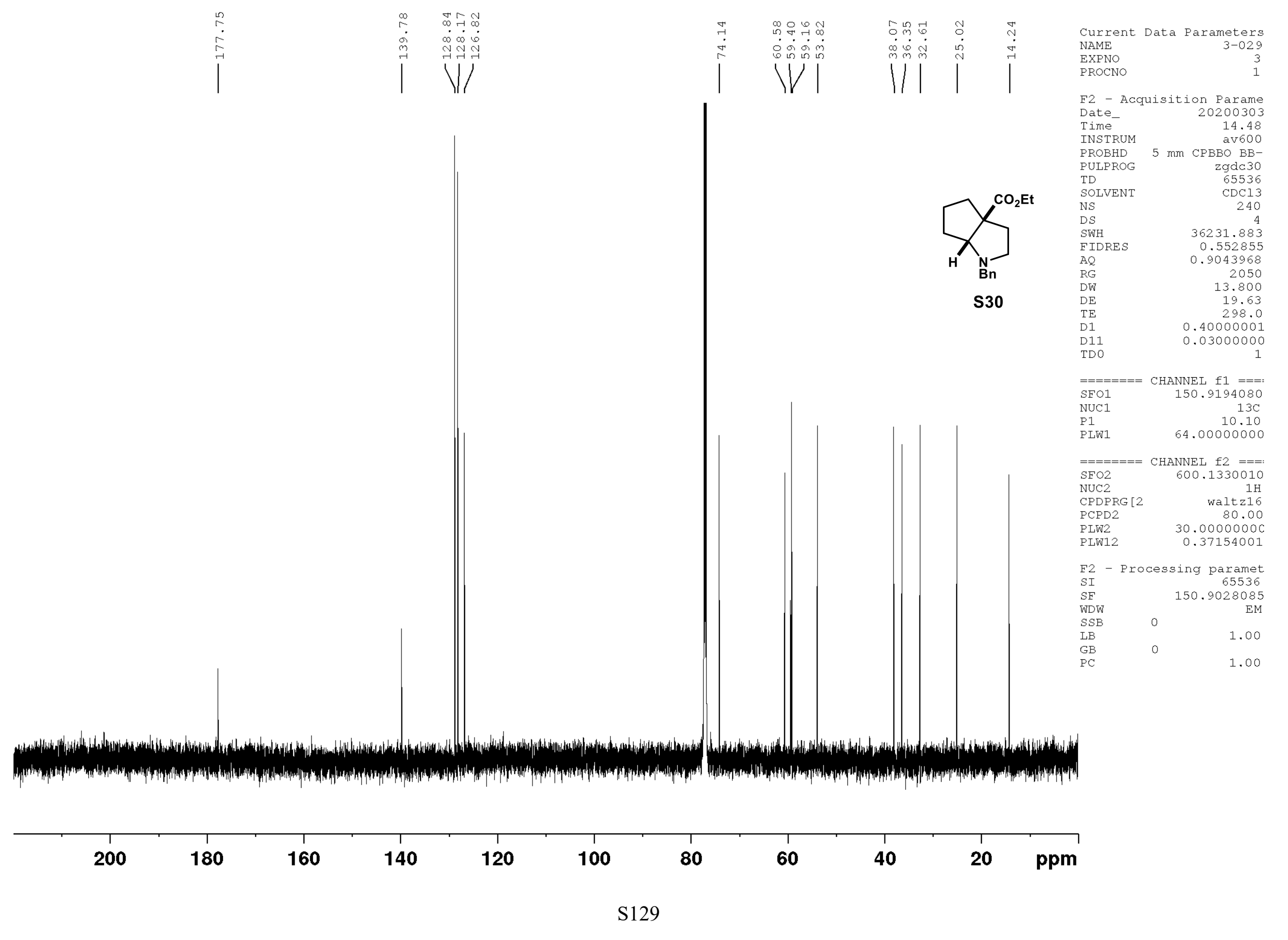




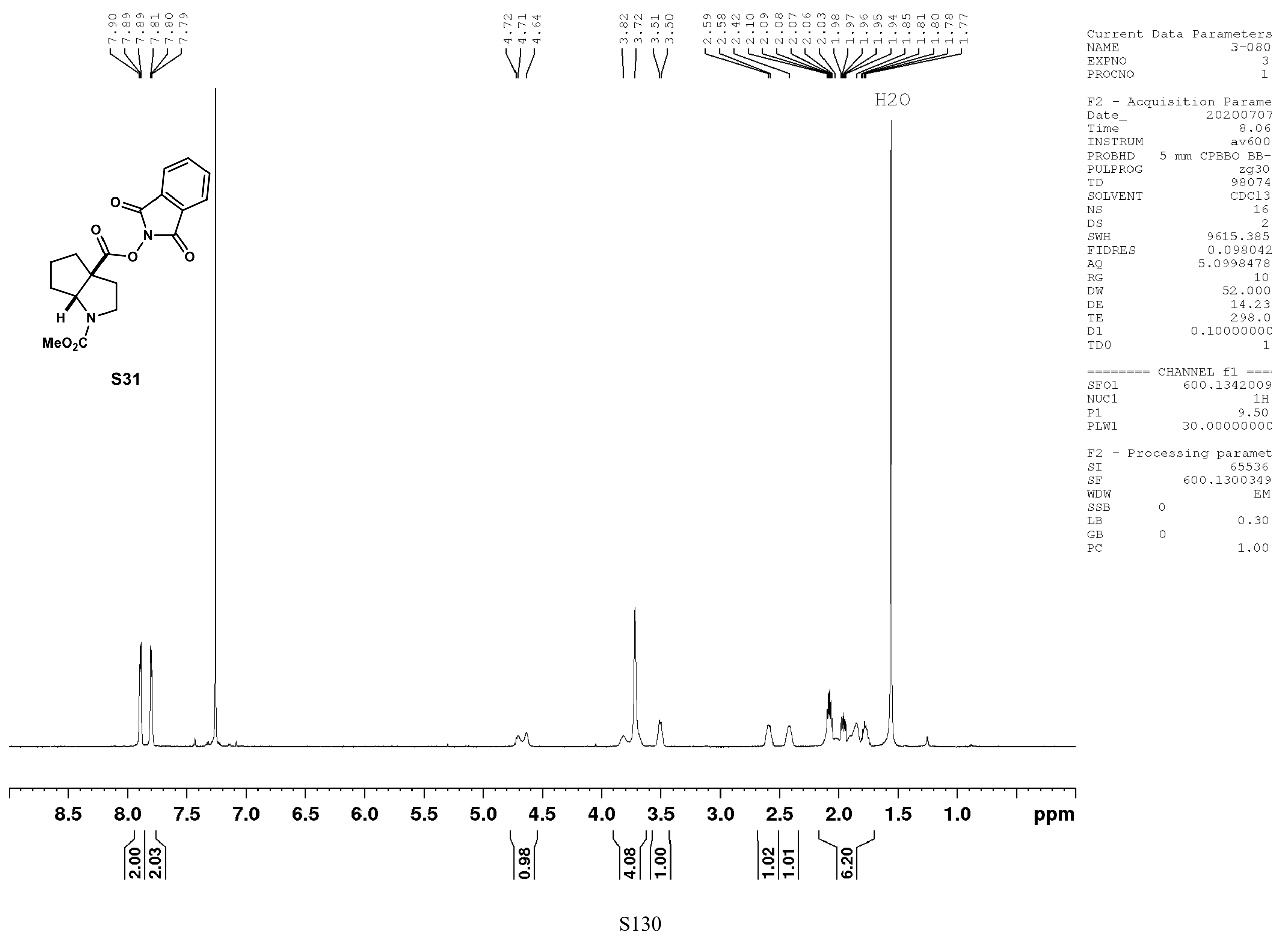




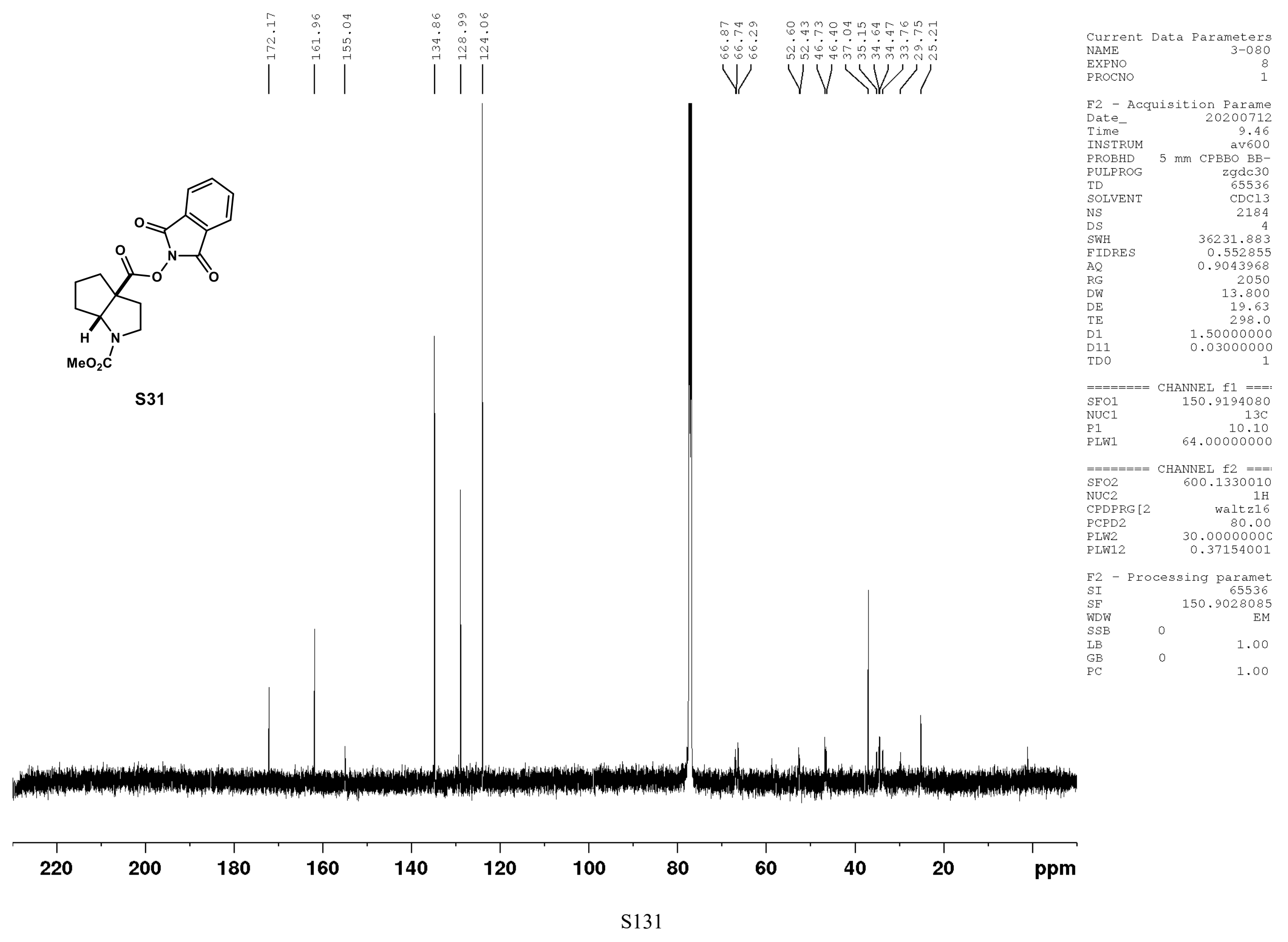

